\title{
Reconstitution of retrotranslocation by the Hrd1 ubiquitin ligase with purified components
}

\author{
Dissertation \\ for the award of the degree \\ "Doctor rerum naturalium" \\ of the Georg-August-Universität Göttingen
}

within the doctoral program Molecular Biology

of the Georg-August University School of Science (GAUSS)

Submitted by

VEDRAN VASIC

from Tuzla, Bosnia and Herzegovina

Göttingen, 2019 

Thesis advisory committee:

Dr. Alexander Stein (Supervisor, Reviewer)

Research Group Membrane Protein Biochemistry

Max Planck Institute for Biophysical Chemistry, Göttingen, Germany

Prof. Dr. Peter Rehling (Reviewer)

Department of Cellular Biochemistry

University Medical Center Göttingen, Germany

\section{Prof. Dr. Claudia Steinem}

Institute of Organic and Biomolecular Chemistry

Georg-August-University Göttingen, Germany

\section{Extended thesis advisory committee:}

Prof. Dr. Michael Meinecke

Department of Cellular Biochemistry

University Medical Center Göttingen, Germany

\section{Dr. Alex Faesen}

Research Group Biochemistry of Signal Dynamics

Max Planck Institute for Biophysical Chemistry, Göttingen, Germany

\section{Prof. Dr. Henning Urlaub}

Research Group Bioanalytical Mass Spectrometry

Max Planck Institute for Biophysical Chemistry, Göttingen, Germany 
I hereby declare that I prepared this doctoral thesis, entitled "Reconstitution of retrotranslocation by the Hrd1 ubiquitin ligase with purified components" on my own and with no other sources and aids than those cited.

Vedran Vasic

Göttingen, April 2019

Parts of this thesis are communicated in the following publication:

Vasic, V.*, Denkert, N.*, Schmidt, C.C., Riedel, D., Stein, A., and Meinecke, M. (2020). Hrd1 forms the retrotranslocation pore regulated by auto-ubiquitination and binding of misfolded proteins. Nature Cell Biology 22, 274-281.

*denotes equal contribution 


\section{Acknowledgements}

This thesis is dedicated to my late father Milo, to my mother Jasmina and to my brother Plamenko. I would like to thank them for their unconditional love and support, which has guided me through the years and will continue to guide me forward in life.

I would like to thank my supervisor, Dr. Alexander Stein, for guiding me through this exciting and ambitious PhD project. His scientific curiosity and optimism know no bounds, and his support for his students and staff is remarkable. I am grateful to my thesis committee members, Prof. Dr. Peter Rehling and Prof. Dr. Claudia Steinem for their input and discussions throughout the course of the project. I would like to thank Dr. Niels Denkert and Prof. Dr. Michael Meinecke for their dedication and input on an exciting collaboration.

I would like to thank my friend and bench mate, Claudia, for her endless input and discussions about my project, and life in general. I am thankful for the wonderful lab atmosphere provided by Anuruti, Aikaterini, Niels, Iris, and Nupur. I am grateful to Nupur and Iris for their assistance in protein purification and cloning, and to Aikaterini, who in her lab rotation helped me with a challenging part of the in vitro reconstitution. I would also like to thank Prof. Dr. Reinhard Jahn and the members of the Department of Neurobiology for their scientific input and friendship over the years.

I am grateful to the Boehringer Ingelheim Fonds for their generous support, for hikes through the Alps, and for bringing me into contact with a wonderful community of passionate scientists. I am also grateful to all the BIF fellows that also became good friends over the years.

I'd like to thank Priyanka, Ridhima, Claudia, Alice, Matt, Benji, Shama, Marc, Justin, Alyssa, JD, Tahere, Hannah, Kashish, Gianmarco, Matteo, Judith, Tomas, and all my other friends in Göttingen and abroad, whose friendships made these last four years very special.

Finally, I would like to thank the staff of the IMPRS for Molecular Biology program, especially Dr. Steffen Burkhardt and Kerstin Grüniger, who have been there from my very first day in Göttingen with unlimited support and guidance. This is truly a special $\mathrm{PhD}$ program, and it is because of their hard work and dedication that this is possible. 



\section{Contents}

$\begin{array}{ll}\text { Abstract } & 6\end{array}$

1 Introduction $\quad 7$

1.1 The endomembrane/secretory pathway . . . . . . . . . . . . 7

1.2 Protein quality control in the ER . . . . . . . . . . . . . . 8

1.2.1 ERAD in Saccharomyces cerevisiae . . . . . . . . . . . 9

1.3 The ubiquitin-proteasome system (UPS) . . . . . . . . . . . . . 11

1.4 Mechanism of ERAD-L in S. cerevisiae . . . . . . . . . . . . . . 13

1.4.1 Delivery of luminal glycosylated substrates to the Hrd1 complex 13

1.4.2 Recognition of non-glycosylated substrates by the Hrd1 complex 15

1.4.3 Insertion of luminal substrates into the Hrd1 complex for retrotranslocation ..................... 16

1.5 Extraction of ERAD-L substrates from the ER membrane by the Cdc48 complex ........................... 20

1.6 End of the road: from Cdc48 to the proteasome . . . . . . . . . . . . 21

1.7 Mechanism of ERAD-M in S. cerevisiae . . . . . . . . . . . . . . . 22

1.8 Role of the Hrd1 complex in mammalian ERAD . . . . . . . . . . . . 23

1.9 The elusive retrotranslocon . . . . . . . . . . . . . . . . . 25

1.10 Proposed model of retrotranslocation by Hrd1 . . . . . . . . . . . . . . 30

1.10.1 Drawbacks of the proposed retrotranslocation model . . . . . . 31

1.11 Open questions about retrotranslocation in ERAD-L . . . . . . . . . 33

1.12 Aims and thesis outline . . . . . . . . . . . . . . . . . 33

2 Materials and Methods $\quad 37$

2.1 Materials . . . . . . . . . . . . . . . . 37

2.1 .1 Lipids . . . . . . . . . . . . . . . . . . 37

2.1.2 Detergents and miscellaneous materials . . . . . . . . . . . . 38

2.1.3 S. cerevisiae strains . . . . . . . . . . . . . . . . . . 39

2.1.4 Bacterial strains . . . . . . . . . . . . . . . . 39

2.1.5 Yeast and bacterial media . . . . . . . . . . . . . . 39 
2.2 Constructs and cloning . . . . . . . . . . . . . . . . . . . . . . . . 39

2.2 .1 Cloning . . . . . . . . . . . . . . . . . . . . . 39

2.2 .2 Plasmids . . . . . . . . . . . . . . . . . . . . 40

2.2.3 Yeast and bacterial transformation . . . . . . . . . . . . 41

2.3 Protein expression . . . . . . . . . . . . . . . . . . . . . . . 41

2.3.1 Protein expression in $S$. cerevisiae . . . . . . . . . . . 41

2.3.2 Protein expression in E. coli . . . . . . . . . . . . . . . . . . . . 42

2.4 Protein purification . . . . . . . . . . . . . . . . . . . . . . . . . . . 42

2.5 Sortase labeling . . . . . . . . . . . . . . . . . . . . . . . . . . . . . 47

2.6 SDS-PAGE and western blotting . . . . . . . . . . . . 47

2.7 Protein reconstitution into liposomes . . . . . . . . . . . . . . 48

2.7.1 Substrate encapsulation into liposomes using the NiNTA method 48

2.7.2 Hrd1 coreconstitution with Syb into liposomes . . . . . . . . . . 49

2.7.3 Reconstitutions of Hrd1, Hrd1/Ubc6 and Ubc6 into liposomes . 50

2.7.4 Nycodenz flotations . . . . . . . . . . . . . . . . . 50

2.7.5 Protease protection assays . . . . . . . . . . . . . 51

2.8 Hrd1 reconstitution into nanodiscs . . . . . . . . . . . . . . . . . 51

2.8.1 Electron microscopy of Hrd1 nanodiscs . . . . . . . . . . . . . 52

2.9 Fluorescence dequenching assay . . . . . . . . . . . . . . . . . . . 53

2.10 Fusion and ubiquitination assays . . . . . . . . . . . . . 53

2.11 Ubiquitination assays with $\mathrm{Hrd1}$ and substrates . . . . . . . . . . . 53

2.12 Pulldown binding assays . . . . . . . . . . . . . . . . 54

2.13 Deubiquitination assays . . . . . . . . . . . . . 55

2.14 Electrophysiological experiments in planar lipid bilayers . . . . . . . . 56

3 Results $\quad 57$

3.1 Developing a fusion system to study retrotranslocation by Hrd1 . . . . 57

3.1 .1 Overview ......................... 57

3.2 Encapsulation of ERAD-L substrates . . . . . . . . . . . . 58

3.2.1 Substrate encapsulation using the traditional method . . . . . . 58

3.2.2 Substrate encapsulation using the NiNTA method . . . . . . . . 61

3.3 Coreconstitution of Hrd1 and Synaptobrevin . . . . . . . . . . . . . 64

3.3.1 Optimization of reconstitution conditions for Hrd1 and Synaptobrevin ......................... 64

3.3.2 Biochemical characterization of Hrd1/Syb liposomes . . . . . . . 67 
3.4 Characterizing fusion between substrate and Hrd1 liposomes . . . . . . 70

3.4.1 Fusion of Hrd1 and substrate liposomes results in efficient membrane mixing . . . . . . . . . . . . . .

3.4.2 Fusion of Hrd1 and substrate liposomes leads to luminal content mixing . . . . . . . . . . . . . . . . . . 74

3.5 Retrotranslocation experiments using the fusion system . . . . . . . . 77

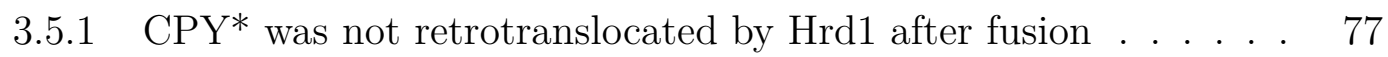

3.5.2 C-terminal tethered CPY* was possibly retrotranslocated . . . . 78

3.5.3 Untethered sCPY*_GFP was not retrotranslocated by Hrd1 . . 80

3.5.4 Fusion causes leakage of substrate . . . . . . . . . . . . 82

3.5.5 Leakage during fusion is dependent on protein:lipid ratio . . . . 83

3.5.6 $\operatorname{PrA}^{*}$ is a Hrd1-substrate in detergent . . . . . . . . . . . . 85

3.5.7 $\operatorname{PrA}^{*}$ does not get retrotranslocated by $\operatorname{Hrd1}$ in the fusion system 86

3.5.8 Retrotranslocation of membrane-anchored ERAD-L substrates . 88

3.6 Bypassing fusion by sequential coreconstitution of Hrd1 and substrate . 89

3.7 Problems with the retrotranslocation experiments . . . . . . . . . . . 91

3.7.1 Aggregation of substrates . . . . . . . . . . . . . . 91

3.7.2 Improving solubility of encapsulated CPY* with urea . . . . . . 92

3.8 Electrophysiological investigation of Hrd1 retrotranslocase activity . . . 95

3.8.1 Reconstitution of Hrd1 into liposomes for PLB experiments . . . 95

3.8.2 Hrd1 forms a dormant channel upon autoubiquitination . . . . . 96

3.8.3 CPY* $^{*}$ stimulates Hrd1 channel activity . . . . . . . . . . . . . . 97

3.8.4 Deubiquitination of Hrd1 closes the channel . . . . . . . . . . . 99

3.8.5 CPY* $^{*}$ stimulates Hrd1 channel activity only from the luminal side101

3.9 Interaction of the cytoplasmic region of Hrd1 with substrates . . . . . . 103

3.9.1 Hrd1 polyubiquitinates cytosolic ERAD-L substrates . . . . . . 103

3.9.2 Polyubiquitinated Hrd1 binds cytosolic CPY* and PrA* . . . . 104

3.9.3 Hrd1 monoubiquitination is not sufficient for cytosolic substrate binding . . . . . . . . . . . . . . 106

3.9.4 CPY $^{*}$ does not bind to polyubiquitin chains alone . . . . . . . . 109

3.9.5 Deubiquitination of Hrd1 partially releases bound substrate . . 110

3.10 Autoubiquitination of the Hrd1 RING domain is essential for substrate binding and channel stability . . . . . . . . . . . . . . . . . . . . . 112

3.10.1 Autoubiquitination of the Hrd1 RING domain is required for efficient cytosolic substrate binding . . . . . . . . . . . . 114

3.10.2 Autoubiquitination of the RING domain is essential for channel stability and substrate activation . . . . . . . . . . . 115 
3.10.3 Lysines in the RING domain are not required for Hrd1 ubiquitin ligase activity . . . . . . . . . . . . . . . . . 117

3.11 Binding of substrates to the luminal side of Hrd1 . . . . . . . . . . 119

3.11.1 Overview of Hrd1 reconstitution into nanodiscs . . . . . . . . . 119

3.11.2 Optimization of nanodisc assembly with Hrd1 and ApoE using DMNG . . . . . . . . . . . . . . . . 120

3.11.3 Reconstitution of Hrd1 into nanodiscs using DM . . . . . . . . . 122

3.11.4 Structural and biochemical characterization of Hrd1 nanodiscs . 124

3.11.5 Hrd1 ubiquitination activity in nanodiscs . . . . . . . . . . . . . 125

3.11.6 $\mathrm{CPY}^{*}$ binds to nonubiquitinated Hrd1 in nanodiscs . . . . . . . 126

4 Discussion $\quad 129$

4.1 The fusion system as a method to study retrotranslocation . . . . . . . 129

4.1.1 Soluble substrates can be efficiently encapsulated into liposomes 130

4.1.2 SNARE-mediated fusion delivers substrates to the luminal side of $\mathrm{Hrd} 1$. . . . . . . . . . . . . . . . . . . . . . . . . . . . . . . 131

4.2 Ubc6 functions as an E2 enzyme for Hrd1 in vitro . . . . . . . . . . . 133

4.3 Potential issues with the fusion system . . . . . . . . . . . . . . . 134

4.3.1 Was Hrd1 inactive or in the incorrect oligomeric state? . . . . . 134

4.3.2 Substrate aggregation . . . . . . . . . . . . 135

4.3.3 Ideas for overcoming substrate aggregation . . . . . . . . . . 136

4.3.4 Retrotranslocation may have occurred but was very inefficient . 137

4.3.5 Is Hrd1 is insufficient for retrotranslocation? . . . . . . . . . . . 137

4.4 Hrd1 forms an ion-conducting channel upon autoubiquitination . . . . 138

4.4.1 Channel closure by deubiquitination . . . . . . . . . . . . . 139

4.5 Autoubiquitination in the Hrd1 RING domain creates a high affinity cytosolic binding site . . . . . . . . . . . . . . . . . . . 140

4.6 Cytosolic binding site may provide the driving force in retrotranslocation 141

4.7 The nature of the conformational change in Hrd1 . . . . . . . . . . . . 142 4.7.1 Hrd1 autoubiquitination may facilitate monomerization . . . . . 144

4.8 Possible mechanisms of cytosolic substrate binding by Hrd1 . . . . . . . 144

4.9 Autoubiquitination of $\operatorname{Hrd} 1$ as an allosteric post-translational modification145

4.10 Substrate binding on the luminal side of Hrd1 . . . . . . . . . . . . 146

4.11 Proposed mechanism of retrotranslocation by Hrd1 . . . . . . . . . . . 147 


\section{List of Figures}

1.1 Protein quality control at the beginning of the endomembrane/secretory pathway . . . . . . . . . . . . . . . . 8

1.2 Overview of ERAD pathways in S cerevisiae . . . . . . . . . 11

1.3 Ubiquitination cascade . . . . . . . . . . . . . . . . . . . . . . 12

1.4 N-glycan processing during protein folding in the ER . . . . . 15

1.5 The Hrd1 complex in ERAD-L . . . . . . . . . . . . . . . . . 17

1.6 Cryo-EM structure of the Hrd1/Hrd3 complex . . . . . . . . . . 30

1.7 Proposed mechanism of Hrd1 retrotranslocation by Baldridge and Rapoport . . . . . . . . . . . . . . . . . . . . . . . . . . . . . . 31

1.8 Reconstitution strategy in the study from Baldridge and Rapoport (2016) . . . . . . . . . . . . . . . . . . . . 32

3.1 Overview of the fusion system f . . . . . . . . . . . . . . . . 58

3.2 General strategy for encapsulation of a molecule into liposomes 59

3.3 Flotation of sCPY*/Syb liposomes . . . . . . . . . . . . . 60

3.4 Protease protection of floated sCPY*/Syb liposomes . . . . . . 60

3.5 Strategy of substrate encapsulation using the NiNTA affinity method . . . . . . . . . . . . . . . . . 62

3.6 Encapsulation efficiency of $\mathrm{His}_{14}-\mathrm{CPY}^{*}$ with increasing concentrations of urea . . . . . . . . . . . . . . . . . 63

3.7 Imidazole flotation and protease protection of $\mathrm{His}_{14}-\mathrm{CPY}^{*} / \Delta \mathrm{N} 49$ liposomes ....................... 64

3.8 Effect of DMNG on Syb reconstitution and fusion . . . . . . . . 66

3.9 Effect of various detergents on SNARE and Hrd1 reconstitution 67

3.10 Flotation and orientation of Hrd1/Syb liposomes . . . . . . . 68

3.11 Coreconstitution efficiency of Hrd1/Syb in liposomes . . . . . . 69

3.12 Hrd1 coreconstitution with Syb does not affect autoubiquitination activity . . . . . . . . . . . . . . . . 70

3.13 Transfer of CPY* to Hrd1 liposomes after fusion . . . . . . . . 72

3.14 Hrd1 autoubiquitinates upon fusion with Ubc6 liposomes . . . 74 
3.15 Fusion system mixes luminal contents efficiently 76

3.16 Encapsulated H14-CPY* does not get retrotranslocated by Hrd1 after fusion .

3.17 C-terminally tagged $\mathrm{CPY}^{*}-\mathrm{H}_{14}$ is efficiently encapsulated into liposomes .

$3.18 \mathrm{CPY}^{*}$-H14 is potentially inefficiently retrotranslocated after fusion

3.19 Encapsulated sCPY*-GFP is not retrotranslocated after cleavage from membrane . . . . . . . . . . . . . . 81

3.20 Ulp1-cleaved sCPY*-GFP leaks out of liposomes during fusion 83

3.21 Leakage occurs during fusion and is dependent on protein:lipid ratio . . . . . . . . . . . . . . . . . . . . 84

$3.22 \operatorname{PrA}^{*}$ is efficiently ubiquitinated by Hrd1 in detergent . . . . . 86

3.23 Encapsulation of $\operatorname{PrA}{ }^{*}$ using the NiNTA method . . . . . . . 87

3.24 PrA* does not get retrotranslocated by Hrd1 . . . . . . . . . . . 88

3.25 Sequential coreconstitution of Hrd1 into CPY* liposomes . . . 90

$3.26 \mathrm{CPY}^{*}$ ubiquitination after sequential Hrd1 coreconstitution . . 91

$3.27 \mathrm{CPY}^{*}$ reconstituted on outside of liposomes is inefficiently ubiquitinated by Hrd1 after fusion . . . . . . . . . . . . . . . . . . 92

3.28 Urea titration of $\mathrm{CPY}^{*} / \Delta \mathrm{N} 49$ liposomes . . . . . . . . . . . . . . 94

3.29 Hrd1 reconstitution into liposomes for planar lipid bilayer experiments ........................ 96

3.30 Hrd1 forms an ion-conducting channel only when polyubiquitinated . . . . . . . . . . . . . . . . . . 97

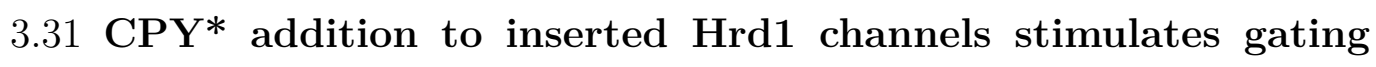
and opening of the channel f . . . . . . . . . . . . . . . . . . . . . 99

3.32 Deubiquitination of Hrd1 leads to channel closure . . . . . . . . 101

3.33 Addition of CPY* to the luminal side of ubiquitinated Hrd1 stimulates channel gating . . . . . . . . . . . . . . . . 102

3.34 Hrd1 polyubiquitinates cytosolic CPY* and PrA* efficiently but not CPY WT and PrA WT . . . . . . . . . . . . . . . 104

3.35 Cytoplasmic region of Hrd1 binds $\mathrm{CPY}^{*}$ upon autoubiquitina-

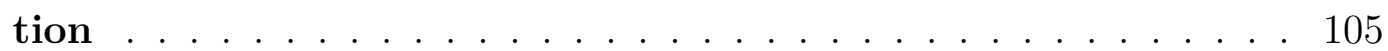

3.36 Titration of ubiquitinated Hrd1 in liposomes against cytosolic $\mathrm{CPY}^{*}, \mathrm{PrA}^{*} \ldots \ldots \ldots \ldots$. . . . . . . . . . 106

3.37 Monoubiquitinated Hrd1 does not bind cytosolic CPY* . . . . 107 
3.38 Hrd1 multi-monoubiquitination by Ubc6 does not a create cytosolic binding site . . . . . . . . . . . . . . . . 109

$3.39 \mathrm{CPY}^{*}$ does not bind to polyubiquitin chains attached to Ubc6 110

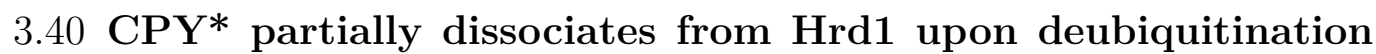
of Hrd1 . . . . . . . . . . . . . . . . . . . . . . . . . . . 111

3.41 Hrd1 lysine to arginine mutants show differential autoubiquitination and CPY* ubiquitination efficiencies . . . . . . . . . . 113

3.42 Quantification of Hrd1 autoubiquitination and CPY* ubiquitination by Hrd1 KxR mutants . . . . . . . . . . . . . . . . . . . . . 114

3.43 Titration of Hrd1 KxR mutants in liposomes against cytosolic $\mathrm{CPY}^{*} \ldots \ldots \ldots \ldots \ldots \ldots \ldots$

3.44 Hrd1 KRK mutant forms highly unstable channels . . . . . . . 117

3.45 Hrd1 ubiquitin ligase activity is maintained in the KRK mutant118

3.46 Reconstitution scheme of Hrd1 into nanodiscs by solubilization of empty liposomes with DMNG . . . . . . . . . . . . . . . 120

3.47 Optimization of ApoE:Hrd1:lipid ratio in nanodisc reconstitutions . . . . . . . . . . . . . . . . . . . 121

3.48 Characterization of lipid and protein content in Hrd1 nanodiscs 122

3.49 Reconstitution of Hrd1 into nanodiscs with DM . . . . . . . . . 123

3.50 Structural and biochemical characterization of Hrd1 nanodiscs 125

3.51 Ubiquitination activity of Hrd1 in nanodiscs . . . . . . . . . . 126

3.52 Differential binding of CPY* to nonubiquitinated and ubiquitinated Hrd1 in nanodiscs . . . . . . . . . . . . . . . . . . . . 127

4.1 NMR Stucture of the Hrd1 RING domain . . . . . . . . . . . . 143

4.2 Updated mechanism of retrotranslocation by Hrd1 . . . . . . . 147 



\section{List of Tables}

1.1 Components of the Hrd1 complex in $S$. cerevisiae and their corresponding mammalian counterparts . . . . . . . . . . 25

2.1 Lipids used in reconstitutions . . . . . . . . . . . . . . . . . . . . . 37

2.2 Detergents used in this thesis . . . . . . . . . . . . . . . 38

2.3 Miscellaneous materials . . . . . . . . . . . . . . . . . . . . . . . . . . 38 


\section{Abstract}

In eukaryotic organisms, a large fraction of newly-synthesized proteins are destined for the endomembrane system or for secretion. The entry gate for these proteins is the endoplasmic reticulum (ER). Proteins are imported into the ER in an unfolded state, where they fold and assemble into their native conformations before being exported to the Golgi apparatus. Proteins which fail to fold or assemble correctly have a propensity to aggregate in the ER, which is toxic for the cell. Accordingly, a quality control pathway termed ER-associated protein degradation (ERAD) recognizes misfolded proteins and retrotranslocates them across the ER membrane into the cytosol, where they are ubiquitinated and degraded by the proteasome.

One of the biggest questions about ERAD is how substrates are retrotranslocated across the ER membrane. Recent evidence implicates one of the central components of ERAD, the Hrd1 ubiquitin ligase, in forming a retrotranslocon. Despite this, it is still unknown if Hrd1 is sufficient for retrotranslocation of luminal ERAD substrates. I created a novel reconstituted system to study retrotranslocation, in which luminal substrates are encapsulated into liposomes and delivered to the luminal side of Hrd1 by SNARE-mediated fusion. The encapsulation was efficient and fusion was shown to mix membrane proteins and luminal contents between liposomes, a technique that has broad applications in membrane protein research. However, retrotranslocation by Hrd1 could not be detected after fusion, which was most likely because of substrate aggregation.

In another set of reconstitutions, Hrd1 reconstituted in nanodiscs bound misfolded proteins on its luminal side, while Hrd1 reconstituted in liposomes bound misfolded proteins with high affinity on its cytosolic side upon autoubiquitination. This was dependent on autoubiquitination in the RING domain. Misfolded proteins could be partially released from the cytoplasmic binding site by deubiquitination. Hrd1 was also incorporated into planar lipid bilayers, and was shown to have channel activity and to conduct ions in a voltage-dependent manner. This was dependent on autoubiquitination in its RING domain. Substrate addition stimulated channel gating and opened the pore to diameters sufficient to fit multiple alpha helices. Remarkably, deubiquitination of Hrd1 closed the channel. Overall, this thesis provides strong evidence that Hrd1 forms a protein-conducting channel in the ER membrane. A model is proposed, whereby an affinity gradient between luminal and cytoplasmic binding sites provides the driving force during retrotranslocation. 


\section{Introduction}

\subsection{The endomembrane/secretory pathway}

Proteins are complex macromolecules consisting of linear chains of amino acids that fold into a three-dimensional shape, which is critical for their function. Proteins perform a plethora of essential functions in the cell, ranging from enzymatic catalysis to signaling and structural support. In the eukaryotic cell, approximately $30 \%$ of all newly-synthesized proteins are targeted to the organelles of the endomembrane system or for secretion into the extracellular space (Ghaemmaghami et al., 2003). This protein trafficking pathway is referred to as the endomembrane/secretory pathway. All proteins destined for the endomembrane/secretory pathway are synthesized by ribosomes in the cytosol and are subsequently threaded into the endoplasmic reticulum (ER) in an unfolded state through the Sec61 forward translocon (Figure 1.1) (Rapoport et al., 2017). The Sec61 translocon is a protein-conducting channel consisting of an aqueous pore, through which proteins containing specialized targeting sequences are imported into the ER (Rapoport et al., 2017). After translocation into the ER, proteins fold into their native structures with the assistance of chaperones, which are specialized proteins that assist in the folding of other proteins (Figure 1.1). The oxidative environment of the ER, along with chaperones called protein disulfide isomerases (PDIs), facilitate the formation of disulfide bonds. Additionally, newly-imported proteins are modified by the attachment of oligosaccharide chains onto asparagine residues, termed N-glycosylation. N-glycosylation serves roles in chaperone binding, increasing solubility, and recognition by quality control machinery in the ER (Braakman and Hebert, 2013). After folding and modification in the ER, proteins are transported to the Golgi apparatus by vesicular traffic, where they are further modified before being routed to their final destination in the cell (Bonifacino and Glick, 2004). 


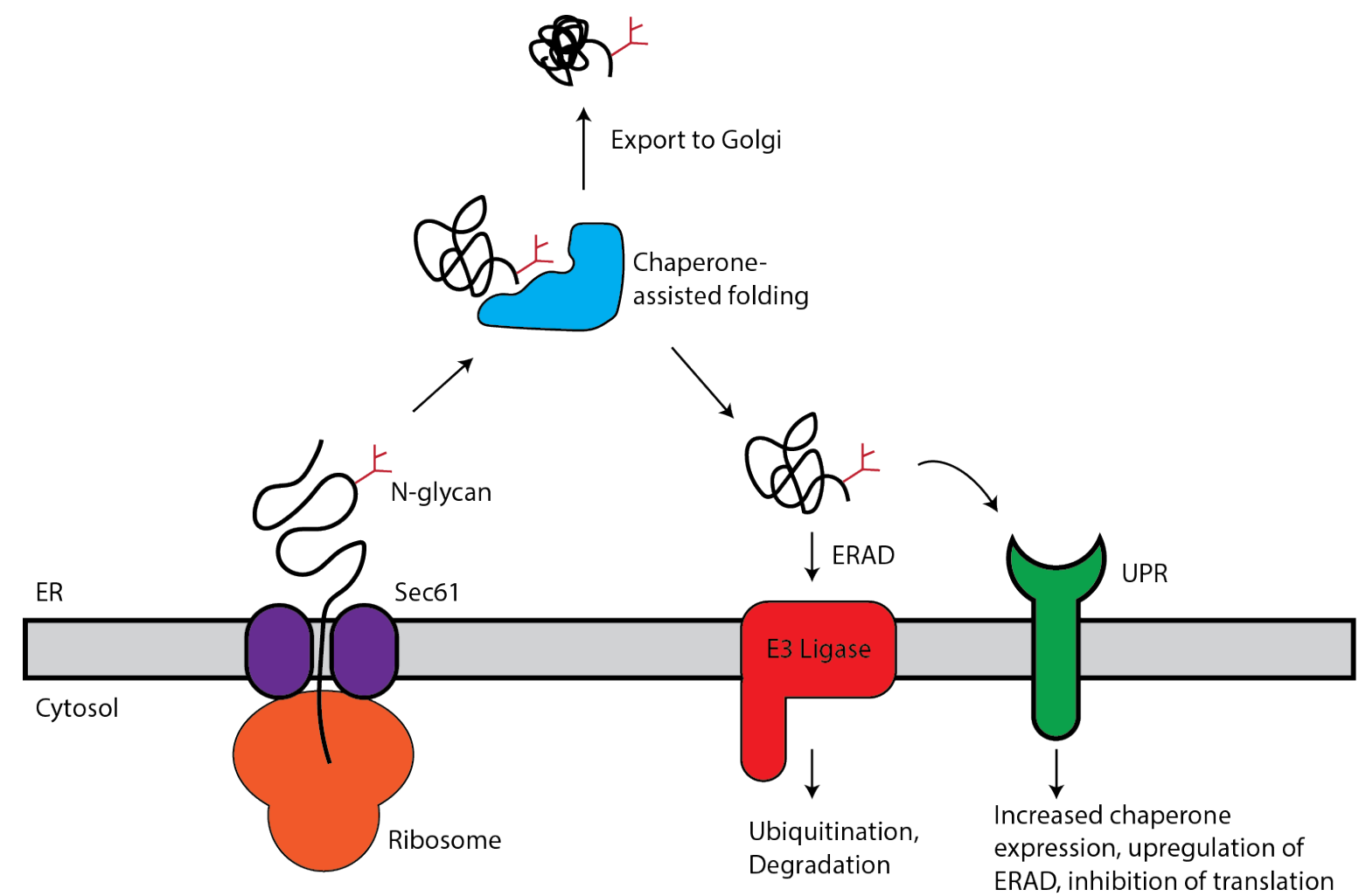

Figure 1.1: Protein quality control at the beginning of the endomembrane/secretory pathway

Newly-synthesized proteins are co-translationally or post-translationally inserted into the ER through the Sec61 translocon. In the ER, they are N-glycosylated and fold with the assistance of chaperones. Correctly-folded proteins are exported to the Golgi apparatus, whereas misfolded proteins are retained in the ER, where they bind to receptors that activate the unfolded protein response (UPR), and are degraded in a process termed ER-associated protein degradation (ERAD). See section 1.2 for details.

\subsection{Protein quality control in the ER}

Protein folding is under strict surveillance in the cell. Protein folding is a stochastic process that proceeds towards the most thermodynamically-stable state (Balchin et al., 2016). This process typically results in the protein achieving its natively-folded state, normally under the assistance of chaperones. However, protein folding is an error-prone process, in which folding intermediates can become kinetically trapped in partiallyfolded states that expose hydrophobic amino acid residues to the aqueous environment of the cell. This is especially relevant in the ER, where important folding steps such as disulfide bond formation and prolyl isomeration are relatively slow, increasing the likelihood that proteins accumulate in partially-folded or misfolded states (Balchin 
et al., 2016). Additionally, external factors such as heat stress, heavy metal stress, oxidation, and mutations can give rise to protein misfolding (Berner et al., 2018). Misfolded proteins have a propensity to irreversibly aggregate, which is toxic for the cell and is linked to numerous diseases including Huntington's disease and Parkinson's disease (Guerriero and Brodsky, 2012). Therefore, protein folding in the ER is overseen by sophisticated quality control mechanisms that remove misfolded proteins before they have the chance to aggregate.

There are two primary quality control systems in the ER (Figure 1.1). The unfolded protein response (UPR) responds to the accumulation of misfolded proteins in the ER through transcriptional responses that upregulate chaperone synthesis, attenuate overall protein translation, and expand the volume of ER (Walter and Ron, 2011; Hetz, 2012). Apart from the UPR, another quality control system of the ER, composed of ubiquitin ligase complexes embedded in the ER-membrane, recognizes and transports misfolded proteins out of the ER and ubiquitinates them on the cytosolic side of the membrane (Mehrtash and Hochstrasser, 2018). Thereafter, these ubiquitinated proteins are extracted from the membrane by the Cdc48 AAA ATPase complex and shuttled to the proteasome for proteolysis (see section 1.3 for an overview of the ubiquitin-proteasome system). This process is termed ER-associated protein degradation, or ERAD. In addition to degrading misfolded proteins, ERAD also degrades orphan subunits of multiprotein complexes and modulates sterol levels by degrading enzymes involved in sterol biosynthesis (Ruggiano et al., 2014). ERAD is also hijacked by viruses to degrade subunits of components involved in immune recognition and by bacterial toxins to enable their retrograde transport out of the ER (Morito and Nagata, 2015). The two ER protein quality control pathways, UPR and ERAD, are linked, with many ERAD components upregulated by the UPR upon ER stress (Hwang and Qi, 2018). In addition key UPR components become ERAD substrates under certain conditions, demonstrating regulatory crosstalk between the two quality control pathways (Hwang and Qi, 2018).

\subsubsection{ERAD in Saccharomyces cerevisiae}

ERAD is conserved across all eukaryotes but is best characterized and understood in the yeast Saccharomyces cerevisiae. In S. cerevisiae, the process is coordinated by two ubiquitin ligase complexes: Hrd1 and Doa10 (Figure 1.2). These complexes coordinate the degradation of specific subsets of substrates, which are defined by the location of their misfolded domain relative to the ER membrane. Substrates of the Hrd1 complex 
contain misfolded domains in the ER lumen or ER membrane, whereas substrates of the Doa10 complex primarily contain misfolded domains in the cytosol, with select substrates also containing misfolded domains in the ER membrane (Carvalho et al., 2006; Vashist and Ng, 2004; Habeck et al., 2015) (Figure 1.2). Consequently, the three pathways are termed ERAD-L (lumen), ERAD-M (membrane) and ERAD-C (cytosol), referring to the location of the misfolded domain. Hrd1 and Doa10 and their associated cofactors are highly conserved from yeast to humans (Christianson and Ye, 2014), indicating the importance of ERAD and the demonstrating the relevance of investigating the molecular mechanisms of ERAD in the model organism S. cerevisiae.

All three pathways can be broken down into the same fundamental steps (Figure 1.2). First, misfolded substrates are recognized by the Hrd1 or Doa10 complexes. Second, substrates containing misfolded domains in the ER lumen are transported across the membrane, or retrotranslocated. Third, the substrates are polyubiquitinated by Hrd1 or Doa10 on the cytosolic side of the membrane, in conjunction with specific E2 enzymes localized at the ER membrane. Fourth, the Cdc48 complex is recruited to the polyubiquitinated substrate, which extracts the substrate from the membrane using rounds of ATP hydrolysis. Finally, the extracted substrate is shuttled to the proteasome, where it is degraded through regulated proteolysis (Berner et al., 2018; Mehrtash and Hochstrasser, 2018).

This thesis deals with the retrotranslocation of misfolded luminal proteins across the ER membrane, as this is one of the most intriguing mechanistic steps in ERAD. Therefore, the introduction focuses on the ERAD-L pathway, dealt with by the Hrd1 complex. ERAD-M, also coordinated by the Hrd1 complex, is discussed in section 1.7. For a detailed review of Doa10-mediated ERAD, the reader is referred to reviews by Zattas and Hochstrasser (2014) and Mehrtash and Hochstrasser (2018). 


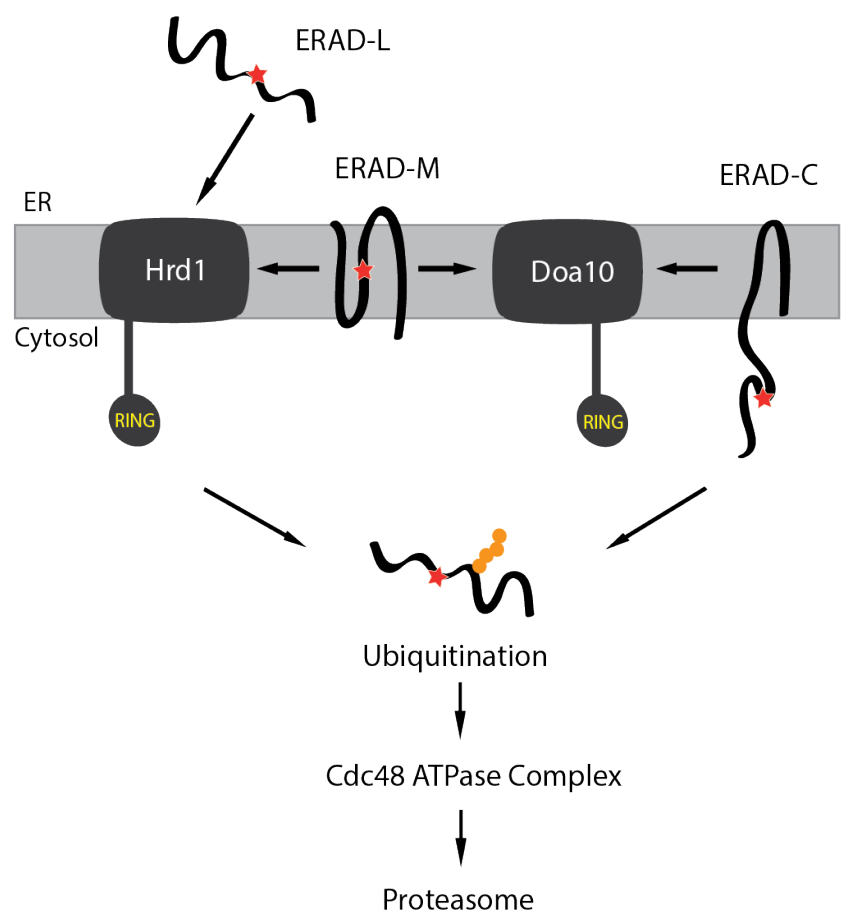

Figure 1.2: Overview of ERAD pathways in $S$. cerevisiae

The three ERAD pathways in S. cerevisiae. The star indicates the misfolded domain. The RING domain is involved in transfer of ubiquitin to the substrate. See text for further details.

\subsection{The ubiquitin-proteasome system (UPS)}

The regulated degradation of proteins in eukaryotic cells is mediated by the covalent attachment of a small, conserved protein called ubiquitin, which marks the protein for proteolysis by by the proteasome. This attachment proceeds through an enzymatic cascade, through which a ubiquitin-activating enzyme (E1), ubiquitin-conjugating enzymes (E2s), and ubiquitin ligases (E3s) coordinate to catalyze the attachment of ubiquitin by formation of an isopeptide bond between the C-terminus of ubiquitin and a lysine residue on the target protein (Komander and Rape, 2012) (Figure 1.3). The attachment of ubiquitin to substrates requires energy. Energy is provided by ATP hydrolysis of the E1, which forms a thioester bond with the C-terminus of ubiquitin, thereby forming an activated ubiquitin-E1 complex (Figure 1.3). The activated ubiquitin is then transferred to an E2 enyzme, which also forms a thioester bond between its active site cysteine and the C-terminus of ubiquitin. Finally, an E3 ligase binds both the substrate and E2 and facilitates transfer of ubiquitin from the E2 to a lysine residue on the substrate (Pickart, 2001). E3 ligases are classified into different families 
based on the type of active site domains they possess. The RING domain ligases are the largest family of E3 ligases. RING E3s catalyze ubiquitin transfer directly from the E2 to the substrate (Komander and Rape, 2012). The two other families of E3 ligases, the HECT and RBR ligases, form a thioester intermediate with ubiquitin before transferring ubiquitin to the substrate (Komander and Rape, 2012).

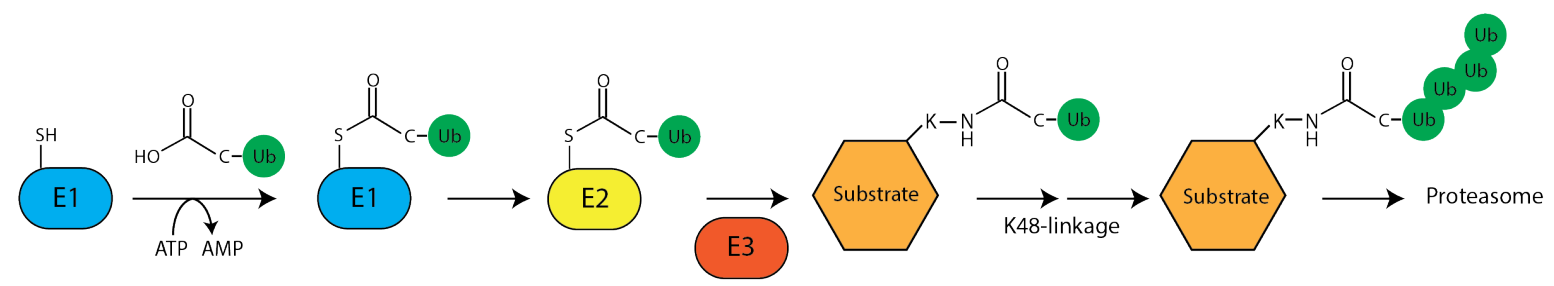

Figure 1.3: Ubiquitination cascade

Ubiquitination proceeds through a cascade of three enzymes termed E1, E2 and E3. Attachment of at least four ubiquitin molecules to the substrate in the form of a K48linked chain targets the substrate for proteasomal degradation. See text for details.

Substrate specificity is determined by the E3 ligases, often in complex with other cofactors (Ravid and Hochstrasser, 2008). This is evident by the large number of E3 ligases compared to E2s and E1s. In S. cerevisiae, there are approximately 60-100 E3 ligases, 11 E2s and only 1 E1 enzyme (Finley et al., 2012). In humans, approximately 40 E2s and at least 600 E3 ubiquitin ligases are predicted to exist (Stewart et al., 2016; Zheng and Shabek, 2017), indicative of the greater substrate complexity in higher eukaryotes. Substrates are recognized through structural elements known as degrons, which are usually hydrophobic in nature and become exposed when proteins dissociate from their binding partners or when they misfold (Ravid and Hochstrasser, 2008).

Ubiquitin can be attached as a single ubiquitin, known as monoubiquitination, or it can be extended to form chains of multiple ubiquitin moeties, known as polyubiquitination. Ubiquitin contains seven lysine residues (K6, K11, K27, K29, K33, K48 and K63), any of which, as well as the free N-terminus, can be used as the attachment site for the next ubiquitin (Komander and Rape, 2012). For RING ligases, the linkage specificity is determined by the E2 enzyme (Komander and Rape, 2012; Stewart et al., 2016). Polyubiquitin chains linked by Lys 48 (K48 chains) are the primary recognition signal for the proteasome (Chau et al., 1989; Pickart, 2001). The proteasome is a large multisubunit, ATP-dependent protease which degrades proteins containing K48 ubiquitin chains. It consists of a 20S core particle and a 19S regulatory particle (Finley, 2009). The $20 \mathrm{~S}$ core particle contains the proteolytic sites, which are found in a narrow constriction in the barrel-shaped core particle (Finley, 2009). The 19S regulatory 
particle is composed two multisubunit assemblies termed the lid and the base. The base contains receptors for K48 ubiquitin chains and binding sites for external ubiquitin adapters, as well as a hexameric AAA ATPase motor, which unfolds substrates and translocates them into the core particle (Bard et al., 2018). The lid functions as a scaffold and contains an essential deubiquitinating enzyme (DUB), which removes ubiquitin chains from the substrate before they enter the AAA ATPase ring (Bard et al., 2018).

\subsection{Mechanism of ERAD-L in $S$. cerevisiae}

\subsubsection{Delivery of luminal glycosylated substrates to the Hrd1 complex}

Recognition of misfolded proteins by the Hrd1 complex is best understood for luminal, $\mathrm{N}$-glycosylated substrates. In particular, two misfolded variants of the yeast vacuolar proteases carboxypeptidase $\mathrm{Y}(\mathrm{CPY})$ and proteinase A (PrA), termed CPY* and $\mathrm{PrA}^{*}$, are substrates of the Hrd1 complex and have been instrumental in determining the molecular mechanisms of ERAD-L (Finger et al., 1993; Hiller et al., 1996; Bordallo et al., 1998). During import into the ER, proteins are N-glycosylated by the oligosaccharyl transferase complex (OST), which is located in the immediate vicinity of the Sec61 translocon. The OST attaches a pre-synthesized Glc $_{3} \mathrm{Man}_{9} \mathrm{GlcNAc}_{2}$ core glycan en bloc to asparagine residues within the NxT consensus sequence (Helenius and Aebi, 2004) (Figure 1.4). The two terminal glucoses are then cleaved by glucosidases in the ER lumen. The monoglucosylated protein becomes a substrate for calnexin and calreticulin, two lectin chaperones in the ER lumen, which are often found in complex with PDIs (Ellgaard and Helenius, 2003). After calnexin and calreticulin-assisted folding, the final glucose residue is cleaved by glucosidase II, producing a Man $\mathrm{GlcNAc}_{2}$ glycan (Man9). An enzyme called UDP-glucose:glycoprotein glucosyltransferase (GT) recognizes partially unfolded regions and reattaches a terminal glucose residue if the protein has not correctly folded (Ellgaard and Helenius, 2003). This reengages the protein with calnexin and calreticulin, which provides it another opportunity to fold correctly. Interestingly, $S$. cerevisiae is the only known eukaryotic species that does not possess the GT enzyme (Parodi, 2000). Thereafter, a mannosidase called Mns1 cleaves off the terminal mannose residue, generating a $\mathrm{Man}_{8} \mathrm{GlcNAc}_{2}$ glycan (Man8). Mns1 is a slow-acting enzyme, which has been suggested to provide proteins a time-window to fold before being routed to the ERAD machinery (Jakob et al., 1998). This hypothesis, 
known as the the mannose timer model, seeks to explain how cells distinguish folding intermediates from misfolded proteins in the ER (Helenius and Aebi, 2004).

The decisive step in whether a protein is exported from the ER or committed to ERAD appears to lie with the removal of an additional mannose to generate a $\mathrm{Man}_{7} \mathrm{GlcNAc}_{2}$ glycan (Man7). This is performed by a mannosidase called Htm1, which is found in complex with PDI (Clerc et al., 2009; Gauss et al., 2011) (Figure 1.4). Htm1 is thought to sense the folding state of proteins through its association with PDI. It preferentially demannosylates Man8 glycans on unfolded polypeptides, and disruption of PDI binding to Htm1 reduces Htm1 mannosidase activity and impairs degradation of ERAD-L substrates (Gauss et al., 2011; Liu et al., 2016). Consistent with this observation, the position of the glycan was found to be important for efficient substrate degradation, indicating that Htm1/PDI recognizes Man8 glycans in a specific structural context, presumably in the vicinity of unfolded regions (Spear and Ng, 2005; Kostova, 2005). The resulting Man7 glycan contains a terminal $\alpha$-1,6-linked mannose, which serves as the recognition signal for Yos9, a lectin which is associated with the Hrd1 complex (Quan et al., 2008). Thus, the Htm1/PDI complex is a surveillance complex that commits Man8-containing glycoproteins with unfolded regions to Hrd1-mediated ERAD. In contrast, correctly-folded proteins containing Man8 glycans avoid the Htm1/PDI complex and are routed to the Golgi by association with sorting receptors in the ER that recognize structural elements and/or glycan signals (Dancourt and Barlowe, 2010; $\mathrm{Xu}$ and $\mathrm{Ng}, 2015)$.

Proteins containing a Man7 glycan are routed to the Hrd1 complex through the lectin Yos9, which recognizes the exposed $\alpha$-1,6-linked mannose through its sugar-binding MRH domain (Buschhorn et al., 2004; Bhamidipati et al., 2005; Kim et al., 2005; Szathmary et al., 2005; Denic et al., 2006; Quan et al., 2008). The substrate is also bound to the ER-luminal Hsp70 chaperone Kar2, along with its Hsp40 cochaperones Scj1 and Jem1 (Plemper et al., 1997; Brodsky et al., 1999; Nishikawa et al., 2001). Yos9 also binds to Kar2 (Denic et al., 2006). Thus, the coordination of Yos9 binding to Man7 glycans and Kar2 binding to unfolded polypeptides acts synergistically to deliver substrates to the Hrd1 complex. 


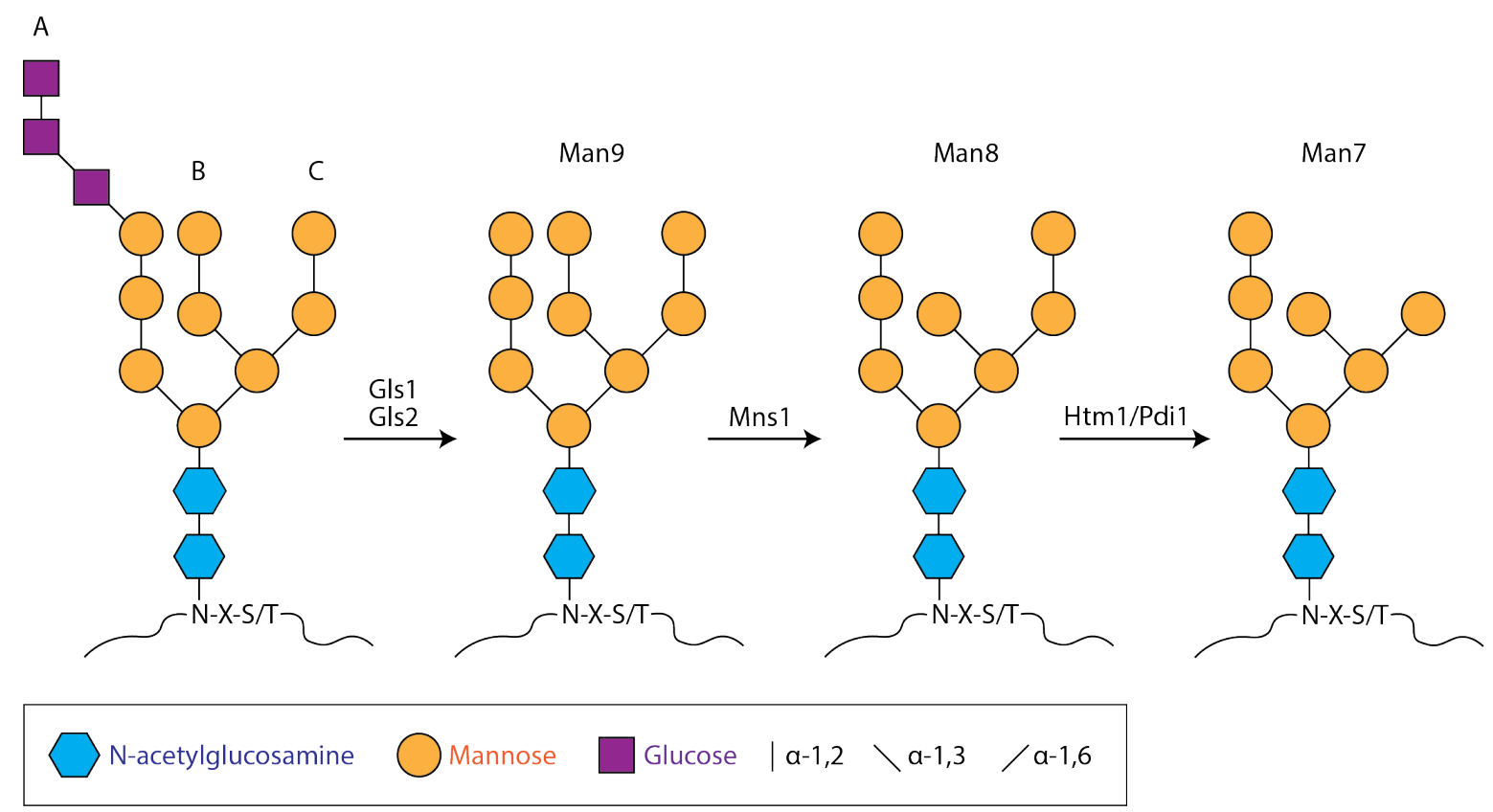

Figure 1.4: N-glycan processing during protein folding in the ER Trimming sequence of N-glycans in the ER lumen. The core N-glycan is attached to an asparagine residue in the substrate and contains three terminal glucose moieties in the A branch, which are cleaved by glucosidases Gls1 and Gls2. The terminal mannose in the B branch is cleaved by Mns1, yielding a Man8 glycan. Misfolded proteins are committed to ERAD by cleavage of the terminal mannose in the $\mathrm{C}$ branch by Htm1/Pdi1, yielding a Man7 glycan. GlcNAc: N-acetylglucosamine. Glc: glucose. Man: mannose. Figure modified from Xu and Ng (2015).

\subsubsection{Recognition of non-glycosylated substrates by the Hrd1 complex}

The ERAD system is also capable of degrading specific types of non-glycosylated substrates. This was demonstrated by the observation that Hrd1 efficiently degrades a variant of $\operatorname{Pr} A^{*}$ where the region containing the glycosylation site is deleted (Kanehara et al., 2010). The glycan independent pathway seems to have different structural determinants for degradation compared to glycan-dependent ERAD (Kanehara et al., 2010). It relies strongly on Kar2 and its Hsp40 co-chaperones, and does not require Yos9 and Htm1 (Kanehara et al., 2010). A similar pathway seems to exist in mammalian ERAD, as a non-glycosylated mutant of $\alpha 1$-antitrypsin (NHK-QQQ) and non-glycosylated, unassembled immunoglobulin $x$ light chains were efficiently degraded and were dependent on BiP, the Kar2 homolog (Hosokawa et al., 2008; Okuda-Shimizu and Hendershot, 2007). Interestingly, non-glycosylated CPY* from S. cerevisiae is ef- 
ficiently degraded in the fission yeast $S$. pombe, and its degradation is not dependent on Htm1 and Yos9 (Mukaiyama et al., 2011). Thus, multiple recognition pathways for ER luminal misfolded proteins exist. It is not clear to which extent they overlap or how substrates are triaged into these different pathways.

\subsubsection{Insertion of luminal substrates into the Hrd1 complex for retrotranslocation}

Misfolded, Man7-glycosylated luminal proteins are delivered to the Hrd1 complex by Yos9 through its interaction with Hrd3, an integral component of the Hrd1 complex (Figure 1.5). Extensive studies using genetic and biochemical methods have identified most, if not all, of the components of the Hrd1 complex required for degradation of an ERAD-L substrate. All proteins shown in Figure 1.5 are required for efficient degradation of misfolded, glycosylated luminal ER proteins. 


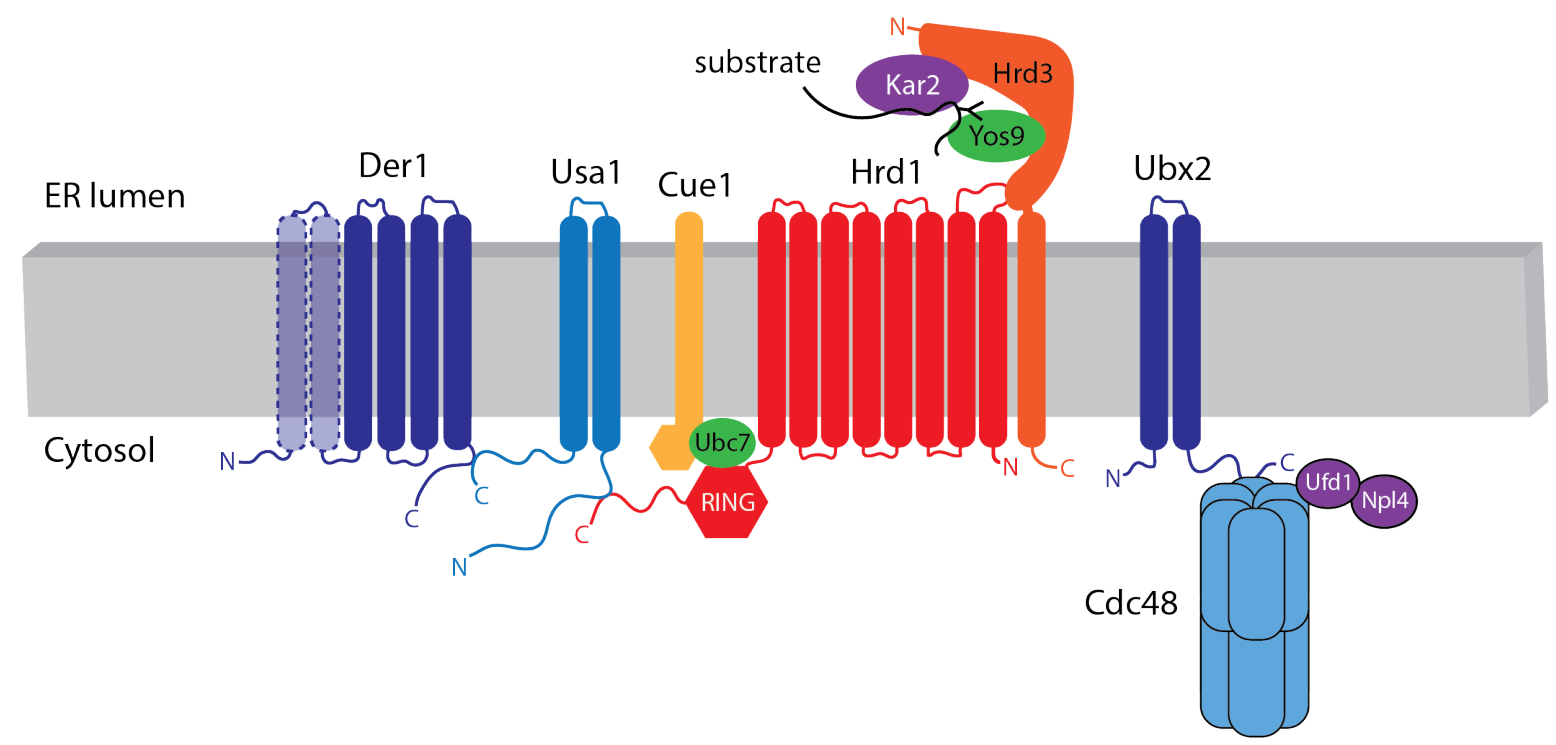

Figure 1.5: The Hrd1 complex in ERAD-L

The necessary components for efficient degradation of misfolded glycosylated proteins are illustrated (ERAD-L pathway). Hrd1, the central component of the complex, is linked to substrates by its interaction with Hrd3. Hrd3 binds to Yos9 and Kar2, capturing the misfolded substrate in the lumen and routing it to Hrd1. Usa1 interacts with Hrd1 to facilitate oligomerization and to link Hrd1 to Der1, which may have a role in inserting substrates into the retrotranslocon. Der1 was shown to have 4 TMs (Hitt and Wolf, 2004), but sequence homology to the mammalian orthologs, the Derlins, indicates that it likely contains 6 TMs (Greenblatt et al., 2011) (indicated by dashed lines). Cue1 recruits the E2 Ubc7, which polyubiquitinates substrates on the cytosolic side of the membrane in conjunction with the Hrd1 RING domain. Ubx2 recruits Cdc48 to the ER membrane, which extracts poylubiquitinated substrates. See text for further details. Figure modified from Zattas and Hochstrasser (2014).

\section{Hrd3}

Hrd3 contains a C-terminal transmembrane domain flanked by a small cytosolic domain and a large luminal domain consisting of 12 Sel1-like repeats (SLRs), which are scaffolding motifs that mediate specific protein-protein interactions (Schoebel et al., 2017; Mittl and Schneider-Brachert, 2007). The most C-terminal SLR of Hrd3 binds to the loop between transmembrane domains (TMs) 1-2 of Hrd1 (Schoebel et al., 2017) (Figure 1.5). Hrd3 is crucial for Hrd1 stability, as Hrd1 rapidly self-degrades due to autoubiquitination in the absence of Hrd3 (Plemper et al., 1999; Gardner et al., 2000; Horn et al., 2009; Vashistha et al., 2016), indicating that Hrd3 influences Hrd1 ubiquitination activity. In addition to its interaction with Yos9, Hrd3 can interact with misfolded proteins independently of Yos9 and likely also with Kar2 and its cochaperone Scj1 (Denic et al., 2006; Gauss et al., 2006; Mehnert et al., 2015), indicating that one of the functions of $\operatorname{Hrd} 3$ is a substrate receptor for the Hrd1 complex. In line with this 
hypothesis, overexpression of Hrd3 inhibits substrate degradation, likely because excess Hrd3 sequesters substrates into dead-end complexes lacking Hrd1 (Plemper et al., 1999). Substrate degradation is restored when Hrd1 is co-overexpressed (Vashistha et al., 2016).

\section{Hrd1}

The Hrd1 ubiquitin ligase is the central component of the Hrd1 complex (Hampton et al., 1996; Bordallo et al., 1998; Gardner et al., 2000). Originally shown to have 6 TMs (Deak and Wolf, 2001), a recent cryo-EM structure of Hrd1 in complex with Hrd3 indicates that it contains $8 \mathrm{TMs}$, with both $\mathrm{N}$ and C-termini facing the cytosol (Schoebel et al., 2017) (see Figure 1.6). Hrd1 has been suggested to form the retrotranslocation channel in ERAD-L (see section 1.9 for a detailed overview). The C-terminal cytoplasmic region of Hrd1 contains a RING H2 domain, which imparts E3 ligase activity, and a long C-terminal extension which is predicted to be largely unstructured (Bordallo and Wolf, 1999; Bays et al., 2001; Deak and Wolf, 2001). The RING domain of Hrd1 catalyzes attachment of K48-linked polyubiquitin chains onto substrates on the cytoplasmic side of the ER, which leads to their extraction from the membrane by the Cdc48 complex and degradation by the proteasome (Bays et al., 2001; Bazirgan and Hampton, 2008). Additionally, Hrd1 autoubiquitinates itself, a function also observed with other E3 ligases (de Bie and Ciechanover, 2011). Autoubiquitination seems to have two functions. First, Hrd1 autoubiquitination in its RING domain is suggested to open the retrotranslocation channel (Baldridge and Rapoport, 2016). Second, it serves as a regulatory mechanism, resulting in Hrd1 degradation by the proteasome (Gardner et al., 2000). Hrd1 functions primarily in conjunction with the cytosolic E2 enzyme Ubc7, and to a much lesser extent with the E2 Ubc1 (Bays et al., 2001; Friedlander et al., 2000). The ER membrane-anchored Ubc6 may also function as an E2 for certain Hrd1 substrates (Hiller et al., 1996).

\section{Usa1}

The C-terminal region of Hrd1 interacts with the N-terminal region of Usa1, a dual membrane spanning protein with $\mathrm{N}$ and C-termini facing the cytosol (Figure 1.5). The N-terminal portion of Usa1 also binds to another Usa1 molecule, thereby facilitating Hrd1 oligomerization, most likely with 2:2 stoichiometry (Carvalho et al., 2006; Horn et al., 2009). Usa1 is required for degradation of ERAD-L substrates and for some ERAD-M substrates (Carvalho et al., 2006; Horn et al., 2009; Carroll and Hampton, 2010). The C-terminal region of Usa1 binds to the C-terminus of Der1, another integral membrane protein of the Hrd1 complex, thereby linking Hrd1 to Der1 (Carvalho et al., 2006; Horn et al., 2009; Mehnert et al., 2013). Usa1 is required in ERAD-L primarily 
due to its role in recruiting Der1 to the Hrd1 complex. Usa1 contains a ubiquitin-like (UBL) domain in its N-terminal region, which seems to play a role in Hrd1 degradation. The UBL domain is required for Hrd1 degradation in the absence of Hrd3, but is not essential for substrate degradation in ERAD-L or ERAD-M (Carroll and Hampton, 2010; Vashistha et al., 2016).

\section{Der1}

Der1 contains 4-6 TMs, with both $\mathrm{N}$ and C-termini facing the cytosol (Hitt and Wolf, 2004) (see section 1.9 for further discussion about the role of Der1 in retrotranslocation). It is exclusively required for ERAD-L but not for ERAD-M (Knop et al., 1996; Vashist and Ng, 2004; Carvalho et al., 2006). As mentioned above, it is linked to the Hrd1 complex through its interaction with Usa1, and is unstable in the absence of Usa1 (Horn et al., 2009). Der1 and its mammalian orthologs, the Derlins (Lilley and Ploegh, 2004; Ye et al., 2004; Oda et al., 2006), share homology to the rhomboid family of intramembrane proteases, but lack the active site residues present in active rhomboid proteases, and likely do not possess intramembrane protease activity (Greenblatt et al., 2011; Freeman, 2014). Although Der1 was found in a previous study to contain 4 TMs (Hitt and Wolf, 2004), its similarity to the 6 TM-containing GlpG and Derlin-1 proteins indicates that it may also contain 6 TMs (Greenblatt et al., 2011; Wang et al., 2006). Der1 interacts with substrates downstream of Hrd3 and Yos9 and has been suggested to insert luminal substrates into the the retrotranslocation machinery (Mehnert et al., 2013) (see section 1.9).

\section{Cue1/Ubc7}

Although technically not part of the Hrd1 complex, Ubc7 is anchored to the ER membrane by Cue1, a single-spanning membrane protein with its soluble region facing the cytosol (Biederer et al., 1997) (Figure 1.5). Cue1 was originally thought to function mainly by increasing the local concentration of Ubc7 at the ER membrane. However, it later became clear that Cue1 is not only a tethering factor of Ubc7, but also significantly stimulates Ubc7-dependent polyubiquitination (Bazirgan et al., 2006; Bazirgan and Hampton, 2008). In fact, tethering Ubc7 to the membrane in the absence of Cue1 is not sufficient for substrate degradation in ERAD-M (Bazirgan and Hampton, 2008). Cue1 contains a Ubc7 binding domain (U7BR) and a ubiquitin binding domain termed a CUE domain (Kostova et al., 2009). The U7BR of Cue1 binds to the backside of Ubc7 while the CUE domain binds preferentially to the distal K48-linked ubiquitin on the substrate. Binding of U7BR to Ubc7 appears to activate Ubc7 for ubiquitin transfer and increases its affinity for the Hrd1 RING domain, while binding of the CUE domain to the distal ubiquitin aligns Ubc7 to efficiently elongate the polyubiquitin 
chain (Bagola et al., 2013; Metzger et al., 2013; von Delbrueck et al., 2016). Therefore, polyubiquitination by Hrd1 and Ubc7 is tightly regulated by Cue1.

\subsection{Extraction of ERAD-L substrates from the ER membrane by the Cdc48 complex}

Once substrates are retrotranslocated through the Hrd1 complex and polyubiquitinated on the cytosolic side of the ER membrane, they are extracted by the Cdc48 AAA ATPase complex (Ye et al., 2001; Jarosch et al., 2002; Rabinovich et al., 2002). The Cdc48 complex consists of the hexameric AAA ATPase Cdc48 and its cofactors Ufd1 and Npl4. AAA ATPases (ATPases associated with a variety of cellular activities) are highly-conserved hexameric protein complexes that harness rounds of ATP hydrolysis to unfold proteins or disentagle protein complexes, a function which is vital in many cell biological processes (Hanson and Whiteheart, 2005). Cdc48 is specialized in extracting ubiquitinated proteins. In addition to ERAD, Cdc48 is involved in the transcriptional regulation of membrane fluidity (Rape et al., 2001; Ballweg and Ernst, 2017), ribosomal quality control (Brandman et al., 2012; Verma et al., 2013), mitochondrial protein quality control (Taylor and Rutter, 2011), and the segregation of proteins from chromatin during mitosis (Ramadan et al., 2007). Cdc48 is a homohexamer consisting of an N-terminal (N) domain and two AAA ATPase domains termed D1 and D2, separated by a small linker. D1 and D2 form two stacked hexameric rings surrounding a narrow pore. The Ufd1/Npl4 (UN) complex binds to the $\mathrm{N}$ domain of Cdc48 and to K48-linked polyubiquitin chains, thereby linking Cdc48 to polyubiquitinated substrates (Bodnar and Rapoport, 2017a).

Cdc48 is recruited to the Hrd1 complex by Ubx2, an integral membrane protein with two TMs, localized to the ER membrane (Neuber et al., 2005; Schuberth and Buchberger, 2005) (Figure 1.5). Ubx2 contains an N-terminal ubiquitin-associated (UBA) domain and C-terminal UBX domain, both localized in the cytosol. The UBX domain binds to the $\mathrm{N}$ domain of Cdc48 while the UBA domain binds to ubiquitinated proteins (Schuberth et al., 2004). Interestingly, both Ubx2 and Ufd1 are required for recruiting Cdc48 to substrates, suggesting a bipartite mechanism of Cdc48 recruitment to the Hrd1 complex (Schuberth and Buchberger, 2005). Ubx2 may act by specifically recruiting the Cdc48 complex to the Hrd1 complex only when Hrd1 is autoubiquitinated or when ubiquitinated substrate is exposed on the cytoplasmic side.

Because the structure of mammalian Cdc48 (p97) shows a very narrow central pore 
(Banerjee et al., 2016), it was unclear if Cdc48 actively unfolds and translocates substrates into the central pore during processing. Recently, the mechanism of Cdc48 extraction was demonstrated for a cytosolic ubiquitinated substrate (Bodnar and Rapoport, 2017b). First, the UN complex binds to the Cdc48 N domain. This binding occurs when the N domain is in an "up" conformation, which is triggered by ATP binding in the D1 ring. Next, UN binds polyubiquitin chains in the substrate, which stimulates ATP hydrolysis in the D2 ring. The substrate is inserted into the central pore and is unfolded during translocation through the pore, which is powered by ATP hydrolysis in the D2 ring. Polyubiquitin chains on the substrate would sterically obstruct translocation through the central pore. They are removed during translocation by the DUB Otu1 (YOD1 in mammals), which binds to the N domain of Cdc48 through its UBXlike domain (Ernst et al., 2009; Bodnar and Rapoport, 2017b). Some oligoubiquitin moieties are retained on the substrate, which are co-translocated through the pore. After translocation, the ubiquitin moieties likely refold and serve as recognition signals for downstream factors, which shuttle the substrate to the proteasome for degradation (Bodnar and Rapoport, 2017a).

In addition to its role in substrate extraction, Cdc48 also acts as a chaperone, maintaining the solubility of extracted proteins in the cytoplasm (Neal et al., 2017). This is especially important for ERAD-M substrates, which are integral membrane proteins containing numerous transmembrane domains.

\subsection{End of the road: from Cdc48 to the proteasome}

After Cdc48 extraction, substrates are recognized by the E4 enzyme Ufd2, an E3 ligase specialized for extending ubiquitin chains (Koegl et al., 1999; Richly et al., 2005). Ufd2 extends the the polyubiquitin chains on the substrate and shuttles the substrate to the adapter proteins Rad23 and Dsk2. Rad23 and Dsk2 interact with Ufd2 and the proteasome through their ubiquitin-like (UBL) domain, and with ubiquitinated substrates through their UBA domains, thereby linking the substrate to the proteasome (Richly et al., 2005; Medicherla et al., 2004; Kim et al., 2004; Wilkinson et al., 2001). Delivery of the substrate to the proteasome by Rad23 and Dsk2 results in its proteolytic degradation.

The enzyme peptide:N-glycanase (Png1, NGLY1 in mammals) removes remaining Nglycans from some substrates before proteasomal degradation. Png1/NGLY1 interacts with Cdc48, Rad23 and the proteasome (Suzuki et al., 2016). The overall importance 
of glycan removal before proteasomal degradation is unclear, as the proteasome is able to degrade N-glycosylated proteins (Kario et al., 2007).

\subsection{Mechanism of ERAD-M in S. cerevisiae}

In ERAD-M, proteins containing misfolded domains in the ER membrane are degraded by the Hrd1 complex. However, the requirements for Hrd1 cofactors differs between ERAD-M and ERAD-L. Most notably, ERAD-M substrates do not require Der1 for their degradation (Vashist and Ng, 2004; Carvalho et al., 2006). Hrd3 is required in ERAD-M due to its role in stabilizing Hrd1, and it also may be important for efficient substrate ubiquitination (Vashistha et al., 2016). Usa1 appears to be dispensable for the degradation of some ERAD-M substrates but important for others (Carvalho et al., 2006; Horn et al., 2009; Carroll and Hampton, 2010). It is not clear why some substrates require Usa1 and others do not. One possibility is that different Hrd1 subcomplexes consisting of $\mathrm{Hrd} 1 / \mathrm{Hrd} 3$ or $\mathrm{Hrd} 1 / \mathrm{Hrd} 3 / \mathrm{Usa} 1$ exist in the cell, and both are involved in ERAD-M. Some ERAD-M substrates may require Hrd1 oligomerization for their degradation, and therefore require Usa1. Those that do not require Hrd1 oligomerization would be degraded by Hrd1/Hrd3 subcomplexes. Downstream of the Hrd1 complex, ERAD-M requires the same ubiquitination and extraction machinery as ERAD-L.

Substrate recognition in ERAD-M is not completely understood, but is thought to be mediated by direct recognition of misfolded membrane segments by the Hrd1 transmembrane domains (Gardner et al., 2001; Sato et al., 2009). Hrd1 might constantly scan the folding status of transmembrane domains in the ER and degrade proteins that contain exposed hydrophilic residues in the membrane, which could be a common feature in membrane misfolding. Consistent with this hypothesis, when conserved hydrophilic residues in the transmembrane domains of Hrd1 were mutated to alanine, degradation of the yeast HMG-CoA reductase enzyme (Hmg2) was abolished (Sato et al., 2009). Mutation of separate, hydrophobic Hrd1 TM residues inhibited degradation of a misfolded plasma membrane transporter $\left(\mathrm{Pdr} 5^{*}\right)$ and a misfolded variant of Sec61 (Sec61-2) (Sato et al., 2009). The conformational changes that expose misfolded transmembrane domains can be externally regulated. For example, degradation of Hmg2 is mediated by the cofactors Nsg1 and Nsg2 (INSIG-1 and 2 in mammals), which bind to Hmg2 in high sterol conditions and stabilize it (Flury et al., 2005; Theesfeld and Hampton, 2013). Conversely, high levels of geranylgeranyl pyrophosphate (GGPP), an isoprenoid intermediate in the sterol synthesis pathway, induce Hmg2 misfolding and 
degradation (Wangeline and Hampton, 2018).

Recently, Dfm1, a Der1 homolog, a was implicated in the retrotranslocation of ERADM substrates (Neal et al., 2018). Dfm1, like Der1, is related to the Derlin family of rhomboid pseudoproteases (Greenblatt et al., 2011). Like Der1, it was predicted to contain 4 TMs (Hitt and Wolf, 2004). However, owing to its similarity to the Derlins, it also likely contains 6 TMs (Greenblatt et al., 2011). Dfm1 contains an SHP box, which binds to Cdc48 and recruits it to the ER membrane (Sato and Hampton, 2006; Goder et al., 2008; Stolz et al., 2010; Neal et al., 2018). Dfm1 also interacts with Doa10 and has been shown to be required for degradation of Ste6*, an ERAD-C substrate (Stolz et al., 2010). Although previous studies showed no effect of Dfm1 deletion on ERAD-L or -M, (Hitt and Wolf, 2004; Sato and Hampton, 2006; Goder et al., 2008; Stolz et al., 2010), Neal et al. showed that Dfm1 was absolutely required for ERAD-M and ERAD-C, but not for ERAD-L. Interestingly, upon substrate overexpression in a Dfm1 deletion background, Hrd1 levels were upregulated by chromosomal duplication, which suppressed the Dfm1 deletion phenotype after multiple generations. This is likely the reason why previous studies overlooked the role of Dfm1 (Sato and Hampton, 2006; Goder et al., 2008). The GxxxG motif and the WR motif, which are essential motifs in rhomboid proteases and pseudoproteases (Greenblatt et al., 2011; Freeman, 2014), were required for substrate degradation. Cdc48 recruitment by the SHP box was also required. The GxxxG motif facilitates membrane protein dimerization (Teese and Langosch, 2015). In line with this, Dfm1 and Der1 form dimers in vivo (Goder et al., 2008; Mehnert et al., 2013). Dfm1 has been hypothesized to form a retrotranslocon or an insertase into the channel for ERAD-M substrates (Neal et al., 2018). Its homology to rhomboid proteases suggests that it contains a hydrophilic cavity and has the ability to perturb the lipid bilayer. Additionally, its ability to dimerize supports the idea that it forms part of a retrotranslocation channel for ERAD-M substrates.

In addition to Dfm1, Hrd1 has also been postulated to form the retrotranslocation channel for ERAD-M. This is further discussed in section 1.9, which deals with the identity of the retrotranslocon in ERAD.

\subsection{Role of the Hrd1 complex in mammalian ERAD}

ERAD is a highly-conserved process across all eukaryotes, which is evident by the presence of mammalian homologs of all components of the S. cerevisiae Hrd1 complex (Table 1.1). Because of the conservation of these components, ERAD likely func- 
tions with similar mechanisms in higher eukaryotes, including mammals. Nevertheless, mammalian ERAD is strikingly more complex than that observed in $S$. cerevisiae.

For one, many more E3 ligases are involved in mammalian ERAD than in S. cerevisiae. In addition to the direct homologs of yeast Hrd1 and Doa10: Hrd1/Synviolin and TEB4/MARCH6, the E3 ligases gp78, TRC8 and RNF145 are also involved in the degradation of different substrates (Christianson and Ye, 2014; Menzies et al., 2018). Interestingly, gp78, TRC8 and RNF145 all show homology to the transmembrane domains of S. cerevisiae Hrd1 (scHrd1) (Schoebel et al., 2017). TRC8 and RNF145 show homology primarily to TMs 3-8 of scHrd1, which are suggested to form the aqueous pore of the retrotranslocon (Schoebel et al., 2017) (see section 1.9 and Figure 1.6). The homology of the mammalian E3 ligases to scHrd1 indicates that they function similarly and probably also form parts of the retrotranslocation machinery. Besides containing regions similar to scHrd1, mammalian ERAD ligases contain additional elements to deal with the increased substrate complexity. Human TRC8 and RNF145 contain sterol-sensing domains, while human gp78 contains a CUE domain and a p97interacting motif, thus combining the function of multiple factors found in $S$. cerevisiae (Christianson and Ye, 2014; Schoebel et al., 2017). Besides the aforementioned E3 ligases, new uncharacterized E3 ligases continue to be discovered in mammalian ERAD (Khouri et al., 2013; van den Boomen et al., 2014; van de Weijer et al., 2014)

In addition to the greater number and complexity of E3 ligases involved in mammalian ERAD, the substrate specificities of different E3 ligases often overlap (Morito et al., 2008; Menzies et al., 2018; Stefanovic-Barrett et al., 2018). Mammalian ERAD appears to be very modular, with various subcomplexes cooperating to degrade specific substrates (Christianson et al., 2011). The substrate overlap and modularity provides more redundancy in the process, which reflects the greater complexity of protein quality control in higher eukaryotes. Nevertheless, although more complex, mammalian ERAD appears to utilize similar molecular mechanisms as observed in S. cerevisiae. 
Table 1.1: Components of the Hrd1 complex in $S$. cerevisiae and their corresponding mammalian counterparts

\begin{tabular}{ccc}
\hline S. cerevisiae & Mammals & Reference \\
\hline Hrd1 & Hrd1/Synviolin & Kikkert et al. (2004) \\
Hrd3 & Sel1L & Lilley and Ploegh (2005); \\
& Mueller et al. (2006) \\
Der1 & Derlin-1, Derlin-2, & Lilley and Ploegh (2004); \\
& Derlin-3 & Ye et al. (2004); Lilley \\
& & and Ploegh (2005); Oda \\
Usa1 & Herp & et al. (2006) \\
& & Schulze et al. (2005); \\
Yos9 & OS-9, XTP3-B & Carvalho et al. (2006) \\
& & Christianson et al. \\
Kar2 & BiP & 2008); Hosokawa et al. \\
& & Christianson et al. \\
Cue1 & & (2008); Hosokawa et al. \\
Ubc7 & AUP1 & (2008) \\
& UBE2G2, UBE2G1 & Mueller et al. (2008) \\
Ubx2 & Kikkert et al. (2004); & Christianson et al. (2011) \\
Cdc48 & UbxD8 & Mueller et al. (2008) \\
Ufd1 & p97/VCP & Ye et al. (2001) \\
Npl4 & UFD1 & Ye et al. (2003) \\
& NPL4 & Ye et al. (2003)
\end{tabular}

\subsection{The elusive retrotranslocon}

The most intriguing question in ERAD is how luminal substrates get retrotranslocated across the ER-membrane in ERAD-L, and what proteins form the retrotranslocon (Hampton and Sommer, 2012). Although detailed genetic and biochemical analysis has provided a clear picture of the components involved, the identity of the retrotranslocon has remained elusive. In the last ten years, evidence has accumulated that suggests that the Hrd1 ubiquitin ligase forms the retrotranslocon in ERAD-L. Other retrotranslocon candidates have been proposed throughout the years, none of which are as compelling as Hrd1. They are briefly summarized below.

\section{Sec61}

Because Sec61 is the forward translocon for protein import into the ER, it was proposed that it may also retrotranslocate substrates into the cytosol during ERAD. 
Studies showed that the ERAD-L substrate CPY* accumulated in Sec61-2 mutant cells (Plemper et al., 1997) and immunoprecipitation experiments demonstrated that Sec61 interacts with components of the Hrd1 complex and with soluble and membranebound ERAD substrates (Wiertz et al., 1996; Schaefer and Wolf, 2009). However, other studies clearly showed no association of components of the Hrd1 and Doa10 complexes with Sec61 in immunoprecipitation and photocrosslinking experiments (Carvalho et al., 2006; Denic et al., 2006; Carvalho et al., 2010; Mehnert et al., 2013).

Sec61 is an essential gene, and therefore studies have relied on temperature-sensitive conditional mutants to study its role in ERAD. These mutants typically have ER import defects, which may have pleiotropic and indirect effects that are difficult to exclude. Retrotranslocation experiments using ER microsomes showed an inhibitory effect of Sec61 cold-sensitive mutants on the degradation of the unglycosylated yeast pro- $\alpha$ factor ( $\Delta \mathrm{gp} \alpha \mathrm{f})$ (Pilon, 1997). However, another study investigating $\Delta \mathrm{gp} \alpha \mathrm{f}$ retrotranslocation in mammalian microsomes showed that retrotranslocation was unaffected when the Sec61 channel was blocked with ribosome-nascent chain complexes (Wahlman et al., 2007). In this study, retrotranslocation was nearly completely blocked when antibodies against Derlin-1 were added to the cytosol (Wahlman et al., 2007). In any case, $\Delta$ gp $\alpha$ is an unusual ERAD-L substrate because it is not ubiquitinated and the Cdc48 complex is dispensable for its retrotranslocation (Werner et al., 1996; Wahlman et al., 2007). Another study using yeast microsomes found no effect of the Sec61-2 temperature-sensitive mutant on Hmg2 retrotranslocation (Garza et al., 2009). One study showed an effect of the Sec61-3 cold-sensitive mutant on CPY* degradation (Willer et al., 2008). However, the Sec61-3 mutant had severe import defects at low temperatures, so a plethora of indirect effects may have contributed to this phenotype. To summarize, it is unclear if Sec61 is directly involved in retrotranslocation. The evidence of Sec61 interaction with the Hrd1 complex is contradictory. Because Sec61 is an essential gene and is also required for ER import, experiments using Sec61 conditional mutants are very difficult to interpret. Only reconstitutions with purified components can conclusively demonstrate whether Sec61 can form a retrotranslocon. As will be shown, together with evidence presented below for Der1 and Hrd1, it is highly unlikely that Sec61 forms the retrotranslocon.

\section{Der1}

Der1, a component of the Hrd1 complex essential for ERAD-L, has been suggested to form part of the retrotranslocation machinery. Photocrosslinking experiments demonstrated that an ERAD-L substrate interacts with both luminal and membrane-embedded residues of Der1 during retrotranslocation, and point mutations in Der1 transmembrane 
domains inhibited substrate degradation (Carvalho et al., 2010; Stanley et al., 2011; Mehnert et al., 2013). It was also shown that Der1 interacts with substrates downstream of Hrd3 and Yos9, and when ubiquitination of Hrd1 was inhibited, substrate accumulated at the Der1 luminal interface (Mehnert et al., 2013). In mammalian ERAD, Derlin-1 is necessary for the retrotranslocation of the luminal substrate NHK $\alpha-1$ antitrypsin and for the membrane substrate MHC class I heavy chain (Greenblatt et al., 2011; Lilley and Ploegh, 2004; Ye et al., 2004). Furthermore, in a mammalian microsome system, retrotranslocation of yeast $\Delta \mathrm{gp} \alpha$ f could be blocked with antibodies against Derlin-1 (Wahlman et al., 2007). Therefore, Der1 likely is directly involved in retrotranslocation.

Although Der1 is clearly involved in the retrotranslocation process, several factors argue against it forming the retrotranslocon exclusively. First, although Der1 oligomerizes (Horn et al., 2009; Mehnert et al., 2013), overexpression of Der1 is not sufficient to overcome the deletion of other factors of the Hrd1 complex (Horn et al., 2009; Carvalho et al., 2010), which would be expected if Der1 was the primary component of the retrotranslocon. Second, Der1 becomes dispensable when Hrd1 is overexpressed (discussed in the following paragraph). Third, analysis of crystal structures of the related bacterial rhomboid protease GlpG indicate that rhomboid proteases are unlikely to form a conduit through the membrane (Wang et al., 2006; Wu et al., 2006). Nevertheless, the structures show a hydrophilic cavity containing the active site dyad, as well as a lateral gate, which allows substrates to enter the cavity from the membrane. It is thus attractive to speculate that Der1 functions as a lateral insertase for the retrotranslocon, perhaps also unfolding substrates before threading them into the channel. Further structural studies and reconstitutions with purified components are needed to determine the exact role of Der1 in retrotranslocation.

\section{Hrd1}

The Hrd1 ubiquitin ligase has emerged as the strongest candidate for the retrotranslocon in ERAD-L. Overexpression of Hrd1 overcomes the simultaneous deletion of Hrd3, Usa1 and Der1 in CPY* degradation (Carvalho et al., 2010). The downstream components including the Cdc48 complex and ubiquitination machinery are still required, indicating that Hrd1 is the only membrane-bound component required for ERADL. In Hrd1 overexpression conditions, substrate N-glycosylation is dispensable (Denic et al., 2006). Additionally, photocrosslinking experiments demonstrated that a luminal ERAD-L substrate interacts with Hrd1 en route to degradation (Carvalho et al., 2010). This interaction is dependent on Hrd1 ubiquitination activity. Interestingly, the N-terminal regions of the substrate interacts with Hrd3 and Der1 while the C-terminal 
region interacts with Hrd1, indicating that the substrate is shuttled from Hrd3 and Der1 to Hrd1 (Carvalho et al., 2010). When ubiquitination of Hrd1 is blocked, substrate accumulates at Hrd3 and Der1 (Mehnert et al., 2013), consistent with the idea that these components act upstream of Hrd1.

Experiments using purified components demonstrated that Hrd1 binds to misfolded proteins through its transmembrane domains, and that oligomerization of Hrd1 increases its affinity towards substrates (Stein et al., 2014). Furthermore, Hrd1 autoubiquitinates, and preferentially polyubiquitinates misfolded proteins in detergent and in proteoliposomes. Polyubiquitination of Hrd1 and substrate recruits the Cdc48 complex, which extracts ubiquitinated Hrd1 and substrate out of the membrane. However, in this study the substrate was fully cleaved by outside proteases after reconstitution, so retrotranslocation could not be concluded (Stein et al., 2014).

A later study utilized proteoliposomes containing purified Hrd1 and a membraneanchored version of $\mathrm{CPY}^{*}$, which allowed for insertion of $\mathrm{CPY}^{*}$ into the liposomal lumen (Baldridge and Rapoport, 2016) (Figure 1.8A). Upon addition of ubiquitination machinery, luminally-oriented $\mathrm{CPY}^{*}$ was retrotranslocated and ubiquitinated by Hrd1. Autoubiquitination of Hrd1 at three critical lysine residues in its RING domain was required for retrotranslocation in the reconstituted system and for degradation of substrates in vivo. Strikingly, ubiquitination of $\mathrm{CPY}^{*}$ was not required for its retrotranslocation, as $\mathrm{CPY}^{*}$ lacking lysines was efficiently retrotranslocated in vitro and degraded in vivo, although it was not ubiquitinated (Baldridge and Rapoport, 2016). However, the study did not demonstrate that Hrd1 and CPY* did not interact in detergent during reconstitution into liposomes. Therefore, it remains a possibility that a non-native retrotranslocation intermediate was inserted into liposomes, which was then released upon Hrd1 autoubiquitination (see section 1.10.1).

A recent cryo-EM structure of the Hrd1/Hrd3 complex illustrates that Hrd1 and Hrd3 form a heterodimer, with two Hrd1 molecules interacting through their transmembrane domains (Schoebel et al., 2017) (Figure 1.6). One molecule of Hrd3 binds to each molecule of Hrd1 in the loop between TMs 1 and 2 in Hrd1, extending as an arch into the luminal side (Schoebel et al., 2017). Hrd1 has eight TMs, instead of the six TMs that were concluded previously (Deak and Wolf, 2001). TMs 3,4 and 6-8 of one Hrd1 molecule form a hydrophilic funnel that is sealed by TM 1 of the adjacent Hrd1 molecule. The luminal side of the funnel is sealed by layers of hydrophobic residues. TM1 could form a lateral gate for substrate entry from the membrane during ERAD-M (Schoebel et al., 2017).

Hrd1 shares similarities to the protein translocases Sec61 and bacterial SecY, which 
also contain a hydrophilic cavity, lateral gate and hydrophobic seal (van den Berg et al., 2004; Wu et al., 2019; Schoebel et al., 2017). Hrd1 also shows similarity to the bacterial membrane protein insertase YidC, which contains a similar hydrophilic cavity (Kumazaki et al., 2014). The structure represents the closed state of the channel, as Hrd1 autoubiquitination is likely required to open the channel (Baldridge and Rapoport, 2016). In addition, ERAD-L requires the full complex consisting of Hrd1, Hrd3, Usa1 and Der1, and it is unclear how these additional cofactors influence the structure of Hrd1. The transmembrane domains of Hrd1 are highly conserved across all eukaryotes. Importantly, other E3 ubiquitin ligases involved in ERAD in higher eukaryotes, such as gp78 and TRC8, share sequence similarity to Hrd1 TMs 3-8, indicating that they can probably also form channels (Schoebel et al., 2017).

In conclusion, a considerable amount of evidence points to Hrd1 forming the retrotranslocon. However, despite all of the evidence presented to date, questions about Hrd1's role in retrotranslocation still remain. These are discussed in the following section. 

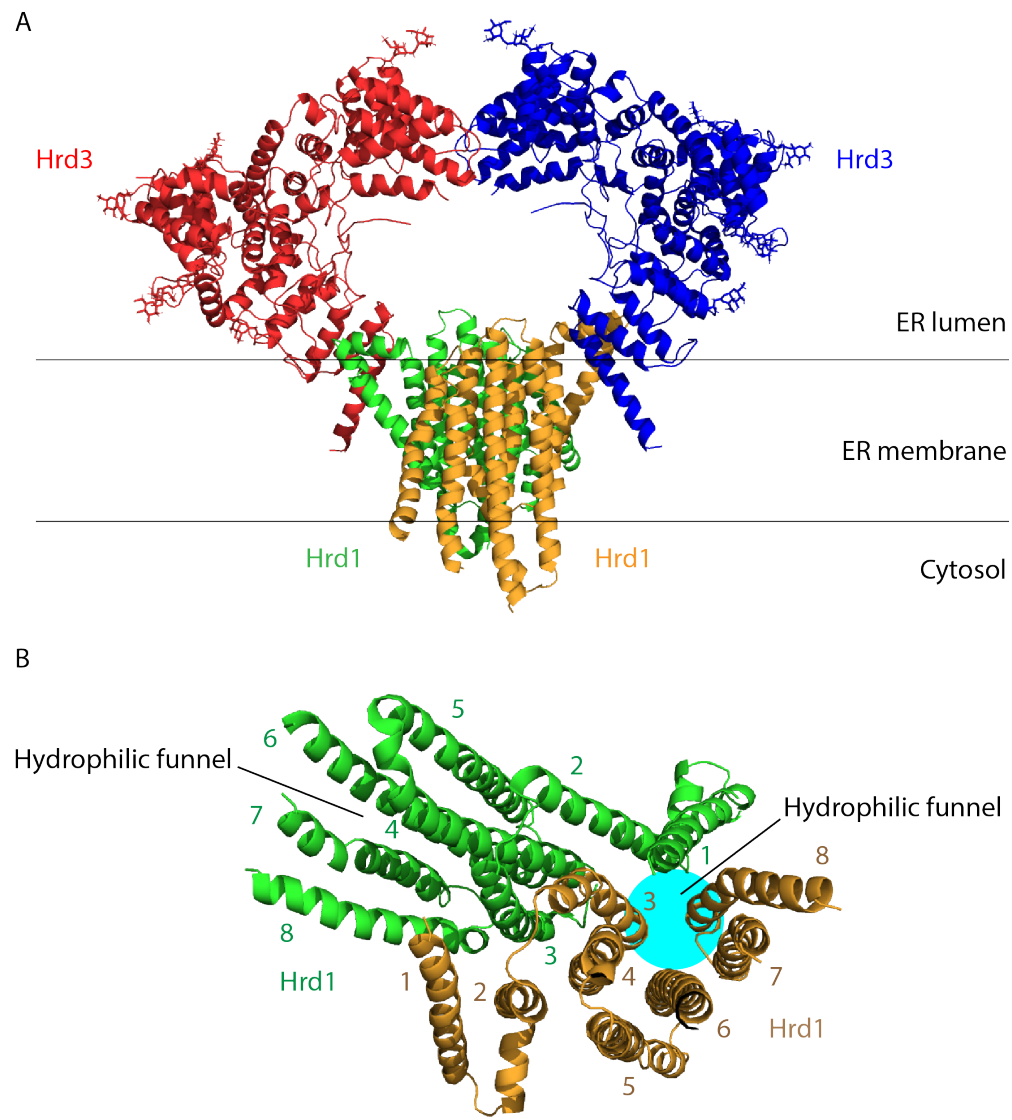

Figure 1.6: Cryo-EM structure of the Hrd1/Hrd3 complex

A: Side view of the Hrd1/Hrd3 complex from within the membrane. Approximate boundaries of the ER membrane are indicated. Hrd1 and Hrd3 form a dimer with 2:2 stoichiometry. Modified from Schoebel et al. (2017). Generated with PyMOL, PDB ID: 5V6P (Hrd1 dimer), 5V7V (Hrd3). B: View of the Hrd1 dimer from the cytosol. The location of the hydrophilic funnel in each Hrd1 molecule, along with the position of the TMs, is indicated. The hydrophilic funnel is formed by TMs 3,4,6, 7 and 8 of one Hrd1 molecule and TM 1 from the other Hrd1 molecule. Modified from Schoebel et al. (2017). Generated with PyMOL, PDB ID: 5V6P.

\subsection{Proposed model of retrotranslocation by Hrd1}

Based on the in vitro reconstitutions of Hrd1 with a membrane-anchored ERAD-L substrate, combined with data from previous reconstitutions (Stein et al., 2014), Baldridge and Rapoport proposed the following model for retrotranslocation by Hrd1 (Baldridge and Rapoport, 2016) (Figure 1.7). First, the substrate (CPY*-TM), containing a mis- 
folded luminal domain, interacts with the luminal side of Hrd1 (steps 1-2). Hrd1 then autoubiquitinates, which opens the channel. The substrate inserts as a loop and slides back and forth in the channel (step 3). The substrate is ubiquitinated by Hrd1 once it reaches the cytoplasmic side, which likely helps to prevent back-sliding into the lumen (step 4). Finally, the Cdc48 complex is recruited, which pulls the ubiquitinated substrate out of the membrane through multiple rounds of ATP hydrolysis (step 5).

1

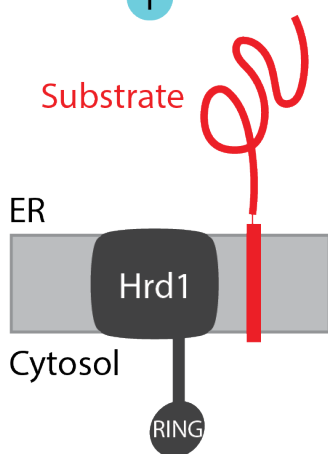

2

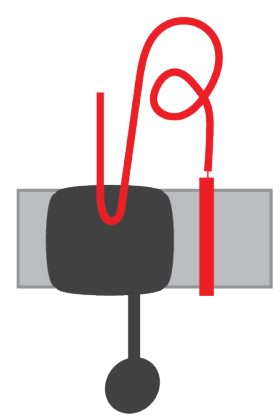

3

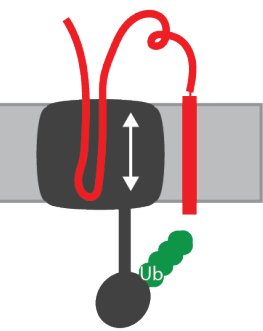

4

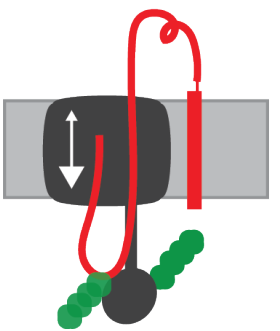

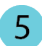

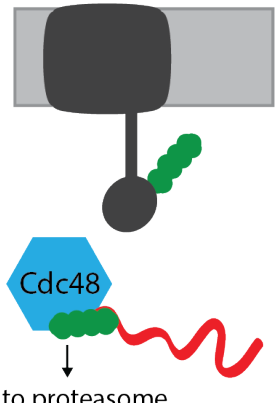


(Baldridge and Rapoport, 2016). In addition, the CPY* appeared to be cleaved from its TM in the ER before degradation, demonstrating that the degradation of a single TM-anchored ERAD-L substrate may be more complex than previously appreciated (Baldridge and Rapoport, 2016).

In order to investigate the mechanism of Hrd1-mediated retrotranslocation in ERAD-L in greater detail, it is therefore essential to reconstitute the process with a fully-luminal misfolded substrate, and to avoid detergent-mediated interactions during reconstitution.

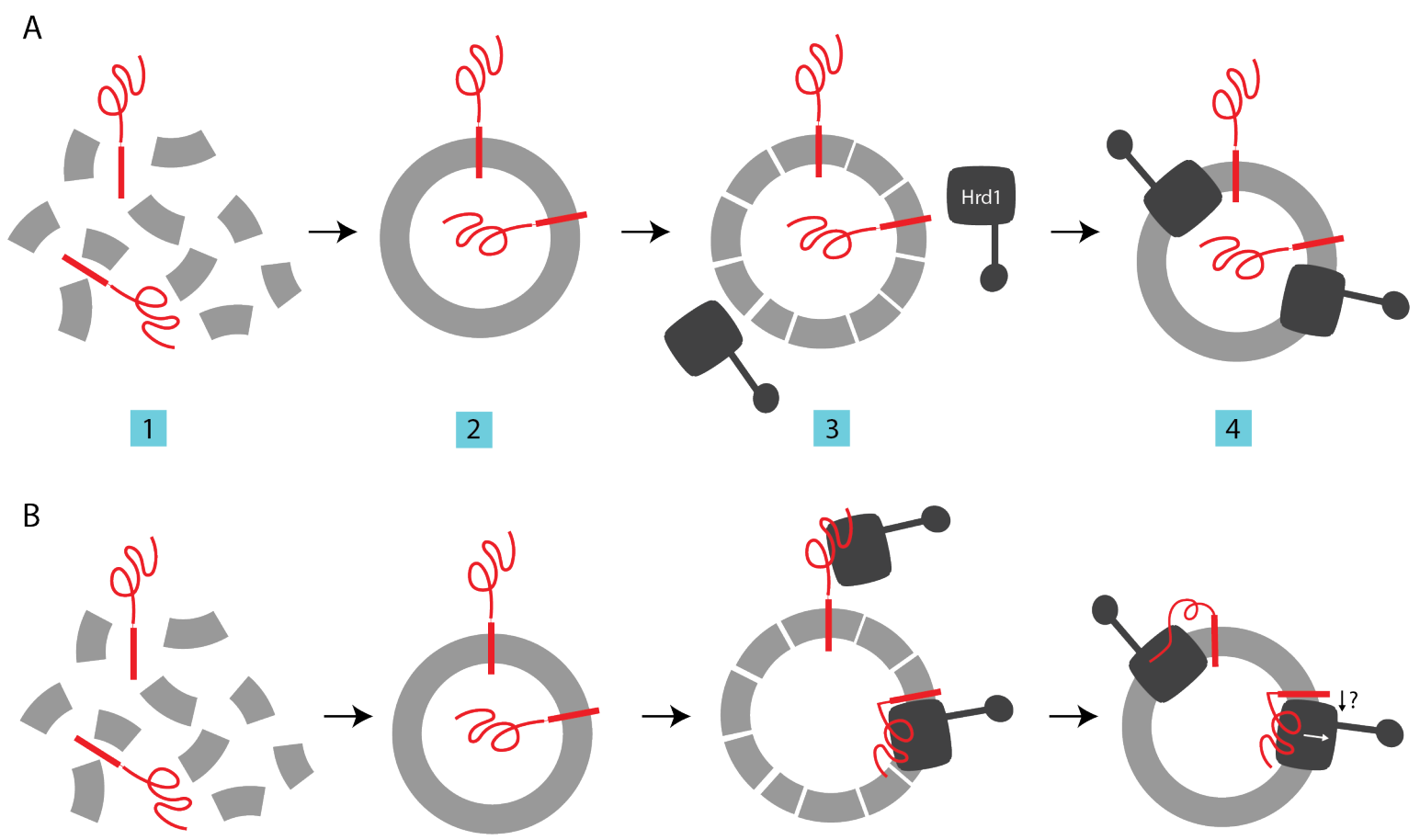

Figure 1.8: Reconstitution strategy in the study from Baldridge and Rapoport (2016)

A: Proposed mechanism of Hrd1 and CPY*-TM coreconstitution as asserted in the study from Baldridge and Rapoport (2016). Step 1: Phospholipids (gray) are solubilized in detergent along with CPY*-TM (red). Step 2: upon detergent removal, CPY*_ TM is inserted bidirectionally into liposomes. Step 3: The liposomes are partially solubilized with limiting amounts of detergent and Hrd1 (black). Hrd1 and CPY*-TM do not interact in detergent. Step 4: Hrd1 is inserted into CPY*-TM liposomes, priming a retrotranslocation reaction of the luminal $\mathrm{CPY}^{*}$ domain. Modified from Baldridge and Rapoport (2016), Figure 2A. B: Alternative mechanism of Hrd1 insertion using the strategy employed above. Steps 1 and 2: same as in A. In step 3, the transmembrane domains of Hrd1 interact with luminal and cytosolic-facing CPY*, mediated by detergent. Step 4: upon detergent removal, luminal $\mathrm{CPY}^{*}$ is partially inserted into the Hrd1 TMs, which may represent an intermediate stage of retrotranslocation. The transmembrane domain of CPY*-TM may also laterally enter into the Hrd1 channel, as indicated. 


\subsection{Open questions about retrotranslocation in ERAD-L}

Based on the evidence presented in section 1.9, my working hypothesis is that Hrd1 forms the retrotranslocon in ERAD-L, while the other components in the Hrd1 complex serve regulatory roles, such as substrate selection, channel gating, and insertion of substrates into the channel. Taking into consideration the model from Baldridge and Rapoport, several open questions remain about the mechanism of retrotranslocation by $\operatorname{Hrd} 1$ :

1. Is Hrd1 sufficient to fully retrotranslocate a luminal substrate across the membrane?

2. Which lysine residues in Hrd1 become autoubiquitinated? How does this autoubiquitination correlate to channel activity?

3. How is directionality provided during retrotranslocation?

4. How does Hrd1 recognize substrates on the luminal side? Which regions of Hrd1 interact with substrates during retrotranslocation?

5. Can Hrd1 accommodate large folded domains or is Cdc48 action required to unfold substrates during retrotranslocation?

In my $\mathrm{PhD}$, I attempted to answer these questions through reconstitution of Hrd1mediated retrotranslocation with purified components. The specific aims and outline of my thesis are presented in the following section.

\subsection{Aims and thesis outline}

Over the last 20 years, most, if not all, of the components involved in ERAD have been unraveled, and a mechanistic understanding of the process is beginning to take shape. Yet, the most glaring gap in our understanding of ERAD lies in one of the most important steps in the process: retrotranslocation of substrates across the ER membrane. Unlike the well-characterized process of forward translocation into the ER, in which newly-synthesized proteins are imported in an unfolded state, retrotranslocation must deal with a diverse range of substrates with partially-folded domains. Therefore, the identity of the retrotranslocon and its mechanism of action are of vital importance in understanding ERAD. 
Genetic and biochemical experiments have implicated the Hrd1 ubiquitin ligase as the retrotranslocon in ERAD-L (Carvalho et al., 2010). Further in vitro reconstitutions using purified components provided strong evidence that Hrd1 can function as a retrotranslocon (Stein et al., 2014; Baldridge and Rapoport, 2016). Yet, as discussed in sections 1.9-1.10, it has not been conclusively demonstrated that Hrd1 can retrotranslocate luminal misfolded proteins across the membrane.

In my $\mathrm{PhD}$, I aimed to reconstitute retrotranslocation by Hrd1 with purified components in various model membranes to determine if Hrd1 is truly sufficient for the retrotranslocation of a fully-luminal substrate. Furthermore, I aimed to characterize the molecular mechanism of retrotranslocation in greater detail. Importantly, reconstitution of protein translocation with purified components is the only method that allows the researcher to answer the question of sufficiency: i.e. what is the minimal machinery required? It is also allows conclusions to be drawn about mechanistic details, because it excludes the multitude of confounding variables present in the cell. Reconstitution with purified components has been used to extensively characterize other translocation events such as forward translocation into the ER (Gorlich and Rapoport, 1993), translocation across the inner bacterial membrane (Brundage et al., 1990; Akimaru et al., 1991), and insertion of membrane proteins into the bacterial outer membrane (Hagan et al., 2010).

The first part of the thesis describes my efforts in developing a novel reconstituted system in proteoliposomes to study retrotranslocation by Hrd1. In order to avoid nonnative detergent-mediated interactions between $\operatorname{Hrd} 1$ and substrate, I reconstituted Hrd1 and the soluble misfolded substrate CPY* in separate populations of liposomes, together with complementary SNARE proteins, which are membrane proteins found in the secretory pathway that catalyze membrane fusion (Jahn and Scheller, 2006). Substrates were delivered to the luminal side of Hrd1 by SNARE-mediated fusion, which faithfully recapitulates the ground state of a retrotranslocation reaction without the presence of detergent. The system is designed to test the sufficiency of Hrd1 in retrotranslocating a fully-luminal substrate across a membrane. The thesis primarily focuses on the development of the system, as the encapsulation of a misfolded protein together with SNAREs, along with the corresponding Hrd1 reconstitutions, was quite challenging and required substantial optimization.

In the next part of my thesis, I collaborated with Prof. Michael Meinecke and Dr. Niels Denkert from the University Medical Center in Göttingen to assay Hrd1 retrotranslocase activity using the planar lipid bilayer (PLB) electrophysiological technique (Harsman et al., 2011). This method provides an alternative readout for activity of 
protein translocases. It takes advantage of the fact that many protein translocases form water-filled pores during translocation, which results in a voltage-dependent ion conductance that can be measured as a readout for channel activity (Harsman et al., 2011). The ion conductance can be used to calculate the pore diameter of the channel. The PLB technique also allowed us to measure channel opening and closing in real time on a single-molecule level. Importantly, this method allowed us to manipulate both sides of the channel, which was not possible in liposomes. We found that Hrd1 formed an aqueous channel upon autoubiquitination. We also characterized substrate interactions with the channel and found that addition of CPY* to the luminal side stimulated channel activity and expanded the pore to diameters large enough to accommodate secondary structures. Finally, we observed closure of the channel upon deubiquitination of Hrd1. Our results demonstrate the first ever ubiquitin-activated channel and illustrate that ubiquitination can act as an allosteric modification in addition to its usual functions.

The final part of the thesis deals with the autoubiquitination sites in Hrd1 and the interaction of substrates with Hrd1. Previous reconstitutions utilized lysine to arginine mutants to determine which residues of Hrd1 are autoubiquitinated (Stein et al., 2014; Baldridge and Rapoport, 2016). However, the mutants showed very small differences in autoubiquitination efficiency in detergent. I reasoned that the difference would become apparent when Hrd1 is reconstituted in a lipid bilayer. I used Hrd1 lysine to arginine mutants reconstituted in liposomes to determine the primary ubiquitination sites.

Previous studies had determined that Hrd1 interacts with misfolded proteins with its transmembrane domains in detergent and that its affinity towards substrate increases upon autoubiqutination (Stein et al., 2014; Baldridge and Rapoport, 2016). However, the transmembrane domains of Hrd1 are occluded by the lipid bilayer in vivo, and the relevant binding sites are on the luminal and cytoplasmic side of the membrane. To study cytoplasmic binding, I reconstituted Hrd1 into liposomes, taking advantage of the unidirectional orientation, with the cytoplasmic domain facing the outside. I determined that a high-affinity substrate cytoplasmic binding site is formed in Hrd1 upon autoubiquitination. In order to study binding on the luminal side of Hrd1, I reconstituted Hrd1 into lipid nanodiscs and determined that Hrd1 binds substrate on the luminal side, but with a lower affinity than the cytoplasmic side. By combining the observations from electrophysiology, autoubiquitination mutants, and side-specific binding experiments, I developed an updated model for retrotranslocation by Hrd1. Our results provide strong evidence to the hypothesis that Hrd1 alone forms the retrotranslocon in ERAD-L. 



\section{Materials and Methods}

\subsection{Materials}

\subsubsection{Lipids}

All lipids were purchased as a lyophilized powder from Avanti Polar Lipids. Prior to reconstitution, the lipids were dissolved in chloroform. Chloroform stocks were stored at $-20^{\circ} \mathrm{C}$ for up to one month.

Table 2.1: Lipids used in reconstitutions

\begin{tabular}{|c|c|c|}
\hline Name & Company & Catalog Number \\
\hline $\begin{array}{l}\text { POPC: 1-palmitoyl-2-oleoyl-glycero-3- } \\
\text { phosphocholine }\end{array}$ & Avanti Polar Lipids & $850457 \mathrm{P}$ \\
\hline $\begin{array}{c}\text { DOPE: 1,2-dioleoyl-sn-glycero-3- } \\
\text { phosphoethanolamine }\end{array}$ & Avanti Polar Lipids & $850725 \mathrm{P}$ \\
\hline $\begin{array}{c}\text { DOPS: } 1,2 \text {-dioleoyl-sn-glycero-3- } \\
\text { phospho-L-serine }\end{array}$ & Avanti Polar Lipids & $840035 \mathrm{P}$ \\
\hline Cholesterol (ovine wool, >98\%) & Avanti Polar Lipids & $700000 \mathrm{P}$ \\
\hline $\begin{array}{l}\text { NBD-PE: 1,2-dioleoyl-sn-glycero-3- } \\
\text { phosphoethanolamine-N-(7-nitro-2- } \\
\text { 1,3-benzoxadiazol-4-yl) }\end{array}$ & Avanti Polar Lipids & $810145 \mathrm{P}$ \\
\hline $\begin{array}{l}\text { Rh-PE: 1,2-dioleoyl-sn-glycero-3- } \\
\text { phosphoethanolamine-N-(lissamine } \\
\text { rhodamine B sulfonyl) }\end{array}$ & Avanti Polar Lipids & $810150 \mathrm{P}$ \\
\hline $\begin{array}{c}\text { NiNTA lipids: } \\
\text { 1,2-dioleoyl-sn-glycero-3-[(N-(5-amino- } \\
\text { 1-carboxypentyl)iminodiacetic } \\
\text { acid)succinyl] (nickel salt) }\end{array}$ & Avanti Polar Lipids & $790404 \mathrm{P}$ \\
\hline
\end{tabular}




\subsubsection{Detergents and miscellaneous materials}

Table 2.2: Detergents used in this thesis

\begin{tabular}{|c|c|}
\hline Name & Company \\
\hline DMNG: Decyl Maltose Neopentyl Glycol & Anatrace \\
\hline DM: n-Decyl $\beta$-maltoside & Glycon Biochemicals \\
\hline Fos-Choline-13, Anagrade & Anatrace \\
\hline Triton X-100: Anapoe-X-100 & Anatrace \\
\hline OG: n-Octyl $\beta$-D-glucopyranoside & Glycon Biochemicals \\
\hline UDM: n-Undecyl ß-maltoside & Glycon Biochemicals \\
\hline DDM: n-Dodecyl $\alpha$-maltoside & Glycon Biochemicals \\
\hline Sodium cholate hydrate, $\geq 99 \%$ & Sigma-Aldrich \\
\hline LDAO: n-Dodecyl-N,N-Dimethylamine-N-Oxide & Anatrace \\
\hline NG: n-Nonyl- $\beta-D-G l u c o p y r a n o s i d e$ & Glycon Biochemicals \\
\hline
\end{tabular}

Table 2.3: Miscellaneous materials

\begin{tabular}{ccc}
\hline Component & Company & Catalog number \\
\hline \hline Antifoam emulsion C & Sigma Aldrich & A 8011 \\
ATP & PanReac AppliChem & A1348 \\
BSA: Bovine serum albumin & PanReac AppliChem & A1391 \\
Coomassie Brilliant blue G-250 & PanReac AppliChem & A3480 \\
DyLight 680 maleimide & Thermo Fisher & 46618 \\
DyLight 800 maleimide & Thermo Fisher & 46621 \\
D(+)-Galactose & PanReac AppliChem & A1131 \\
HEPES & Carl Roth & 6763 \\
HisPur NiNTA resin & Thermo Fisher & 88223 \\
Imidazole & Sigma Aldrich & I202 \\
IPTG, Dioxane free & Formedium & IPTG025 \\
D-mannitol & Sigma Aldrich & M9647 \\
Nycodenz & Abbott Diagnostics & 1002424 \\
Pepstatin A & Peptide Institute & 4397 \\
Proteinase K & Jena Bioscience & EN-178S \\
PMSF: Phenylmethyl sulphonyl fluoride & Carl Roth & 6367 \\
Pierce Detergent removal spin columns & Thermo Fisher & 87777 \\
Pierce High capacity streptavidin agarose & Thermo Fisher & 20361 \\
Pierce Streptavidin magnetic beads & Thermo Fisher & 88817 \\
TCEP & Sigma Aldrich & C4706 \\
Ubiquitin (WT) & Boston Biochem & U-100Sc \\
Ubiquitin (K48R) & LifeSensors & SI217 \\
YEP broth & Formedium & CCM0410 \\
\hline
\end{tabular}




\subsubsection{S. cerevisiae strains}

Hrd1 and all Hrd1 lysine mutants were expressed in a ubc7 deletion strain derived from BY4742 (OpenBiosystems) (MAT $\alpha$ ura3 $\Delta 0$ his $3 \Delta 1$ leu2 $\Delta 0$ lys2 $\Delta 0$ ubc7::KANR) (Stein et al., 2014). The ERAD substrates CPY*, PrA*, and their WT forms were expressed in a hrd3, alg3 double deletion strain, derived from BY4741 (OpenBiosystems) (MATa ura3 $\Delta 0$ his3 $\Delta 1$ leu2 $\Delta 0$ lys2 $\Delta 0$ hrd3::KANR alg3::HIS3) (Stein et al., 2014). The E1 enzyme Uba1 was expressed in the InvSc1 strain (Invitrogen).

\subsubsection{Bacterial strains}

The human Usp2 catalytic domain, ApoE422k, and the following proteins from S. cerevisiae: sCPY*, sCPY*-GFP, Ubc7, Ubc6, the cytoplasmic domain of Cue1 (Cue1-c), Ulp1, and the Doa10 RING domain, were all expressed in the E. coli strain BL21CodonPlus (DE3)-RIPL (Agilent). The neuronal SNARE synaptobrevin 2, the soluble domain of synaptobrevin 2 , and the acceptor SNARE complexes $\Delta$ N49 and $\Delta$ N53 (all from Rattus norvegicus) were also expressed in BL21-CodonPlus (DE3)-RIPL (Agilent).

\subsubsection{Yeast and bacterial media}

For normal maintenance and growth, E. coli cultures were grown in LB medium supplemented with the appropriate antibiotics (final concentrations: $40 \mu \mathrm{g} / \mathrm{ml}$ kanamycin, $100 \mathrm{\mu g} / \mathrm{ml}$ ampicillin, $37 \mathrm{\mu g} / \mathrm{ml}$ chloramphenicol) (Miller, 1992). For protein expression, E. coli strains were grown in terrific broth (TB) supplemented with the appropriate antibiotics (Tartoff and Hobbs, 1987). S. cerevisiae strains containing transformed plasmids were grown in synthetic complete (SC) medium containing $2 \%$ (w/v) glucose and the corresponding amino acid/uracil dropouts (Burke et al., 2005). S. cerevisiae deletion strains were grown in YPD medium (Burke et al., 2005).

\subsection{Constructs and cloning}

\subsubsection{Cloning}

All cloning was performed by either Gibson assembly or site-directed mutagenesis. Gibson assembly was used for inserting fragments into vectors and swapping fragments 
between vectors. Overlapping primers for Gibson assembly were designed using the NEBuilder assembly tool and the cloning was performed with the NEB HiFi assembly mix, according to manufacturer's instructions (New England BioLabs). For mutagenesis, inserting sequences less than 100 base pairs, and deleting sequences from existing vectors, the Q5 site-directed mutagenesis kit was used (New England BioLabs), according to manufacturer's instructions. Primers for site-directed mutagenesis were designed using the NEBaseChanger tool (New England BioLabs).

\subsubsection{Plasmids}

Proteins expressed in S. cerevisiae were cloned into galactose-inducible expression vectors of the pRS series (Mumberg et al., 1994), as in Stein et al. (2014). Hrd1 was expressed in pRS426-PGal1, and contained a C-terminal streptavidin-binding peptide tag lacking lysines (SBP), a tobacco etch virus (TEV) cleavage site before the SBP tag, and a C-terminal LPETGG tag for sortase-mediated labeling with fluorescent dyes (Stein et al., 2014; Popp and Ploegh, 2011). CPY* contained the N-terminal signal sequence from $S$. cerevisiae prepro- $\alpha$-factor and the G255R point mutation (Finger et al., 1993). It was cloned into pRS425-pGal1, with a C-terminal His ${ }_{14}$-LPETGG tag preceded by an HA tag for detection by western blotting. Finally the construct contained an HDEL sequence for ER retention (Pelham et al., 1988). Alternatively, a $\mathrm{CPY}^{*}$ construct containing an N-terminal His ${ }_{14}$-tag followed by a $3 \mathrm{C}$ protease cleavage site was cloned into the same vector. CPY WT, in pRS425-PGal1, contained an Nterminal prepro- $\alpha$-factor signal sequence followed by an N-terminal $\mathrm{His}_{14}-3 \mathrm{C}$ tag and a C-terminal LPETGG-HDEL tag. The gene encoding PrA (pep4), was amplified from genomic DNA and cloned by Gibson assembly into pRS426-PGal1 with C-terminal His $_{14}$ and LPETGG tags. To create PrA*, the sequence encoding amino acids 54-92 was removed by site directed mutagenesis (Spear and Ng, 2005). The plasmids encoding CPY*-TM and CPY*-TM2, both in pRS426-PGal1, were kindly provided by Dr. Ryan Baldridge (Baldridge and Rapoport, 2016). The plasmid encoding the E1 enzyme Uba1 was as described in Stein et al. (2014).

The proteins Ubc7 and Cue1-c were expressed in the vectors pET28b and pET21b, respectively, as previously described (Stein et al., 2014). Ubc6, Ubc6-SBP, sCPY*_ GFP and the Doa10 RING domain (amino acids 1-129) were expressed in the K27 SUMO vector, which contains an N-terminal His ${ }_{14}$-SUMO tag (Stein et al., 2014). Ubc6 constructs were kindly provided by Claudia C. Schmidt (Max Planck Institute for Biophysical Chemistry). The expression vector containing Usp2 was a gift 
from Cheryl Arrowsmith (Addgene plasmid \# 36894 ; http://n2t.net/addgene:36894 ; RRID:Addgene_36894). Ulp1, with an N-terminal His ${ }_{14}$-TEV sequence, was expressed in a vector derived from pQE-80L (Qiagen), provided kindly by Prof. Dirk Görlich, Max Planck Institute for Biophysical Chemistry. Synaptobrevin was expressed in the pET28a vector as previously described (Pobbati et al., 2006), whereas the soluble domain of synaptobrevin $\left(\mathrm{Syb}_{\mathrm{sol}}\right.$, amino acids 1-95) was expressed in K27SUMO. The $\Delta \mathrm{N} 49$ and $\Delta \mathrm{N} 53$ complexes were expressed in the pETDuet-1 vector, as previously described (Stein et al., 2007; Pobbati et al., 2006). sCPY*, containing an N-terminal His $_{14}$-SUMO tag and a C-terminal LPETGG tag, was expressed in a $\mathrm{pBAD} / \mathrm{HisC}$ vector (Invitrogen). The plasmid encoding ApoE422K was a gift from Oscar Bello and James Rothman (Bello et al., 2016). The sequence encoding ApoE422K was cloned into the K27SUMO vector.

\subsubsection{Yeast and bacterial transformation}

Yeast transformation was carried out as described in Burke et al. (2005) under "Highefficiency Transformation of Yeast". Transformed yeast were grown on SC agar plates with the appropriate dropout for three days at $30^{\circ} \mathrm{C}$ and colonies were restreaked on fresh SC plates. Yeast strains were stored in $25 \%(\mathrm{w} / \mathrm{v})$ glycerol at $-80^{\circ} \mathrm{C}$. Bacterial transformation was performed as described in Miller (1992). Transformed bacteria were grown on LB plates with appropriate antibiotics (concentrations listed in section 2.1.5). For protein expression, bacteria were transformed freshly before each expression.

\subsection{Protein expression}

\subsubsection{Protein expression in S. cerevisiae}

Protein expression in $S$. cerevisiae by galactose induction was performed essentially as described in Stein et al. (2014), with some minor modifications. Briefly, a starter culture of the transformed yeast strain was innoculated in SC medium with the appropriate dropout. The starter culture was grown at $30^{\circ} \mathrm{C}$ for $24 \mathrm{~h}$ and was diluted 1:50 into 6 $\mathrm{L}$ of fresh medium. This was grown for an additional $24 \mathrm{~h}$ at $30^{\circ} \mathrm{C}$ and expression was induced by adding a $4 \mathrm{X}$ stock of induction medium ( $8 \%$ galactose $(\mathrm{w} / \mathrm{v})$ in $12 \%$ YEP $(\mathrm{w} / \mathrm{v})$ broth with $1 \%(\mathrm{v} / \mathrm{v})$ antifoam $\mathrm{C}$ emulsion (Sigma)), giving a final concentration of $2 \%(\mathrm{w} / \mathrm{v})$ galactose. The cultures were grown for $16 \mathrm{~h}$ at $30^{\circ} \mathrm{C}$ and harvested by 
centrifugation at $3000 \mathrm{x}$ g. The cells were washed with MilliQ water, resuspended in a minimal amount of MilliQ water, and stored at $-80^{\circ} \mathrm{C}$ until further use.

\subsubsection{Protein expression in E. coli}

Protein expression in E. coli was performed as in Stein et al. (2014). Briefly, a streak of colonies was innoculated into LB medium supplemented with the appropriate antibiotics (including chloramphenicol for the BL21-CodonPlus RIPL strains), and the preculture was grown for $16 \mathrm{~h}$ at $37^{\circ} \mathrm{C}$. The preculture was diluted 1:50 into TB medium containing the appropriate antibiotics, but without chloramphenicol. The cultures were grown to an $\mathrm{OD}_{600}$ of $0.8-1$ and expression was induced with $0.5 \mathrm{mM}$ isopropyl $\beta$-D-1thiogalactopyranoside (IPTG). After induction, the cultures were grown at for $16 \mathrm{~h}$ at $18^{\circ} \mathrm{C}$ and were havested by centrifugation at $3000 \mathrm{x}$ g. The pellet was resuspended in a minimal volume of nickel wash buffer $(50 \mathrm{mM}$ Tris- $\mathrm{HCl} \mathrm{pH} 8.0,500 \mathrm{mM} \mathrm{NaCl}, 40$ $\mathrm{mM}$ imidazole (for $\mathrm{His}_{14}$-tagged proteins)). The imidazole concentration was reduced to $8 \mathrm{mM}$ for $\mathrm{His}_{6}$-tagged proteins. The resuspended pellet was stored at $-20^{\circ} \mathrm{C}$.

\subsection{Protein purification}

All protein purification steps were performed at $4^{\circ} \mathrm{C}$ or on ice, except where indicated otherwise.

Hrd1: Hrd1 and Hrd1 mutants were purified as in Stein et al. (2014), with the following modifications in the membrane fraction preparation. Hrd1 expression was induced at $25^{\circ} \mathrm{C}$ instead of $30^{\circ} \mathrm{C}$. Approximately $100-150 \mathrm{~g}$ of cells were resuspended in $900 \mathrm{ml}$ of cold MilliQ water. $2 \mathrm{mM}$ DTT was added the cells were incubated on ice for 15 min. After centrifugation at $3000 \mathrm{x} g$ for $10 \mathrm{~min}$, the pellet was resuspended in buffer $\mathrm{M}_{\mathrm{F}}$ (20 mM HEPES-KOH pH 7.5, 5 mM KoAc, 600 mM mannitol, 0.5 mM EDTA). 1 $\mathrm{mM}$ phenylmethanesulfonyl fluoride (PMSF) and $2 \mathrm{mM}$ pepstatin A were added fresh. Lysis was performed in a bead beater (BioSpec) with zirconia glass beads, with the following cycles: $15 \mathrm{~s}$ on, $1 \mathrm{~min}$ off, for a total of $50 \mathrm{~min}$. The beads were separated by filtration and the lysate was centrifuged for at $1500 \mathrm{x}$ g for 10 min to pellet unbroken cells. The supernatant was then centrifuged at 40,000 x g for $45 \mathrm{~min}$ in a Ti45 rotor (Beckman). The resulting supernatant was recovered and was centrifuged at 40,000 rpm for $30 \mathrm{~min}$ in a Ti45 rotor. The resulting pellet was dounced and resuspended in a minimal volume of buffer $\mathrm{M}_{\mathrm{F}}$. This is referred to as the crude membrane fraction. 
Membrane fractions were flash frozen and stored at $-80^{\circ} \mathrm{C}$. Hrd1 was purified from the crude membrane fraction as previously described (Stein et al., 2014).

$C P Y^{*}$ : $\mathrm{CPY}^{*}$ was purified as follows. A crude membrane fraction was prepared as previously described (Baldridge and Rapoport, 2016), with the exception that $2 \mu \mathrm{M}$ pepstatin A was used and the centrifugation steps were performed at 40,000 rpm in a Ti45 rotor. $\mathrm{CPY}^{*}$ was purified from the membrane fraction as follows, modified from the purification of CPY*-TM in Baldridge and Rapoport (2016). The membrane fraction was washed with buffer $\mathrm{M}_{\mathrm{C}}(50 \mathrm{mM}$ HEPES-KOH $\mathrm{pH} 7.4,300 \mathrm{mM} \mathrm{KCl}$ ) and was pelleted by centrifugation at 40,000 rpm for $30 \mathrm{~min}$ in a Ti45 rotor. The resulting pellet was resuspended in $250 \mathrm{ml}$ of buffer $\mathrm{S}_{\mathrm{C}}(50 \mathrm{mM}$ HEPES-KOH pH 7.4, $300 \mathrm{mM} \mathrm{KCl}, 1 \mathrm{mM} \mathrm{MgAc}, 1 \mathrm{mM}$ TCEP, $40 \mathrm{mM}$ imidazole, $6 \mathrm{M}$ urea) and CPY* was solubilized by stirring for $1 \mathrm{~h}$ at $4^{\circ} \mathrm{C}$. The lysate was cleared by centrifugation at 40,000 rpm for $30 \mathrm{~min}$ in a Ti45 rotor. The cleared lysate was loaded onto a HisTrap HP 5 $\mathrm{ml}$ column (GE Healthcare), which was pre-equilibrated with buffer $\mathrm{S}_{\mathrm{C}}$. After binding, the column was washed with $30 \mathrm{CVs}$ of buffer $\mathrm{W}_{\mathrm{C}}(25 \mathrm{mM}$ HEPES-KOH pH 7.4, $300 \mathrm{mM} \mathrm{KCl}, 1 \mathrm{mM} \mathrm{MgAc}, 0.5 \mathrm{mM}$ TCEP, $40 \mathrm{mM}$ imidazole, $2 \mathrm{mM}$ Fos-choline-13), which replaced urea for the harsh detergent Fos-choline. Elution was carried out with buffer $\mathrm{I}_{\mathrm{C}}(25 \mathrm{mM}$ HEPES-KOH pH 7.4, $300 \mathrm{mM} \mathrm{KCl,} 1 \mathrm{mM} \mathrm{MgAc}, 0.5 \mathrm{mM}$ TCEP, $400 \mathrm{mM}$ imidazole, $2 \mathrm{mM}$ Fos-choline-13). The eluted CPY* was analyzed by SDSPAGE, and peak fractions were pooled and concentrated with an Amicon filtration unit (30 kDa, MilliPore). $\mathrm{CPY}^{*}$ was sortase labeled with a fluorescent dye and was further purified by gel filtration on a Superdex 200 increase 10/300 GL column (GE Healthcare) equilibrated with buffer $\mathrm{L}_{\mathrm{C}}(20 \mathrm{mM}$ HEPES-KOH pH 7.4, $300 \mathrm{mM} \mathrm{KCl}$, $1 \mathrm{mM}$ magnesium chloride, $1 \mathrm{mM}$ DTT, $2.5 \mathrm{M}$ urea).

$C P Y$ WT: CPY WT was purified directly from the lysate without the preparation of a membrane fraction. Briefly, the cell pellet was resuspended in buffer $\mathrm{M}_{\mathrm{Y}}(50 \mathrm{mM}$ HEPES-KOH pH 7.4, $300 \mathrm{mM} \mathrm{KCl,} 30 \mathrm{mM}$ imidazole). PMSF (1 mM) and pepstatin A $(2 \mu \mathrm{M})$ were added fresh, and lysis was performed using a bead beater, as described in the Hrd1 purification. To remove unbroken cells, the lysate was centrifuged at 2000 $\mathrm{x} g$ for $10 \mathrm{~min}$. The resulting supernatant was centrifuged at 40,000 rpm for $30 \mathrm{~min}$ in a Ti45 rotor. To further clarify the lysate, the resulting supernatant was centrifuged again at 40,000 rpm for $30 \mathrm{~min}$. A white film of lipids was found in the supernatant, which could be removed by filtration through a $0.45 \mu \mathrm{m}$ filter. The clarified lysate was incubated with $6 \mathrm{ml}$ of HisPur resin (Thermo Scientific) for $3 \mathrm{~h}$ at $4^{\circ} \mathrm{C}$, with rotation. The lysate was transferred to a column and was washed with 65 column volumes (CVs) of buffer $\mathrm{M}_{\mathrm{Y}}$. The protein was eluted with buffer $\mathrm{I}_{\mathrm{Y}}$ (50 mM HEPES-KOH pH 7.4, 
$300 \mathrm{mM} \mathrm{KCl}, 400 \mathrm{mM}$ imidazole) and peak fractions were pooled and concentrated in an Amicon filter (30 kDa cutoff). Further purification was achieved by gel filtration on a HiLoad Superdex 200 16/60 column (GE Healthcare) equilibrated with buffer LY (20 mM HEPES-KOH pH 7.4, 150 mM KCl, 5 mM $\beta$-mercaptoethanol). Purified CPY WT was sortase-labeled and further purified on a Superdex 200 increase 10/300 GL column with buffer $\mathrm{L}_{\mathrm{Y}}$.

$\operatorname{Pr} A^{*}$ : $\operatorname{Pr} A^{*}$ was purified essentially as $\mathrm{CPY}^{*}$, with the following exceptions. Following solubilization and binding to the HisTrap column in $6 \mathrm{M}$ urea buffer, the urea concentration was reduced to $2 \mathrm{M}$ for the elution step: buffer $\mathrm{I}_{\mathrm{P}}(50 \mathrm{mM}$ HEPES-KOH pH 7.4, $300 \mathrm{mM} \mathrm{KCl,} 40 \mathrm{mM}$ imidazole, 0.5 mM TCEP, $2 \mathrm{M}$ urea). PrA* was further purified by gel filtration on a HiLoad Superdex 200 16/60 column equilibrated with buffer $\mathrm{L}_{\mathrm{P}}(50 \mathrm{mM}$ HEPES-KOH pH 7.4, $300 \mathrm{mM} \mathrm{KCl,} 0.5 \mathrm{mM}$ TCEP, 2 M urea). Afterwards, it was sortase-labeled and further purified on a Superdex 200 increase 10/300 GL column equilibrated with buffer $\mathrm{L}_{\mathrm{P}}$.

PrA WT: PrA WT was purified exactly as CPY WT. Sortase labeling and further purification was done as with CPY WT.

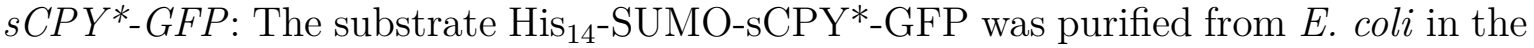
following manner. The cell pellet was resuspended in approximately $200 \mathrm{ml}$ of nickel wash (50 mM Tris-HCl pH 7.4 at RT, $500 \mathrm{mM} \mathrm{NaCl,} 40 \mathrm{mM}$ imidazole). PMSF (1 mM) and pepstatin A $(2 \mu \mathrm{M})$ were added freshly and the cells were lysed with two passes through a microfluidizer at 17,000 PSI (M-110L, Microfluidics). Urea was added in powder form to the lysate to a final concentration of $2 \mathrm{M}$, which prevented the protein from aggregating on the nickel beads. After stirring for $30 \mathrm{~min}$, the lysate was cleared by centrifugation at 14,000 rpm in a F14S-6x250y rotor (Thermo Scientific) for 30 min. The supernatant was incubated with $8 \mathrm{ml}$ of HisPur resin for $3 \mathrm{~h}$ with rotation. The beads were washed with $40 \mathrm{CVs}$ of buffer $\mathrm{W}_{\mathrm{S}}(50 \mathrm{mM}$ Tris- $\mathrm{HCl} \mathrm{pH} 8.0,500 \mathrm{mM}$ $\mathrm{NaCl}, 40 \mathrm{mM}$ imidazole, $2 \mathrm{M}$ urea, $5 \mathrm{mM} \beta$-mercaptoethanol). The protein was eluted with buffer $\mathrm{I}_{\mathrm{S}}(20 \mathrm{mM}$ Tris- $\mathrm{HCl} \mathrm{pH} 8.0,500 \mathrm{mM} \mathrm{NaCl}, 400 \mathrm{mM}$ imidazole, $2 \mathrm{M}$ urea, $5 \mathrm{mM}$ beta-mercaptoethanol) and was further purified by gel filtration on a HiLoad Superdex 200 26/60 column in buffer $\mathrm{I}_{\mathrm{S}}$ without imidazole. Finally, the protein was sortase-labeled and further purified on a Superdex 200 increase 10/300 GL column.

sCPY*: His 14 -SUMO-sCPY*-LPETGG, without the GFP tag, was purified in a different manner, optimized by Claudia C. Schmidt (Max Planck Institute for Biophysical Chemistry). Expression from the pBAD vector was induced with $0.2 \%(\mathrm{w} / \mathrm{v})$ L-arabinose for $16 \mathrm{~h}$ at $23^{\circ} \mathrm{C}$. The cell pellet was resuspended in buffer $\mathrm{M}_{\mathrm{S}}(50 \mathrm{mM}$ Tris-HCl pH 8.0, $500 \mathrm{mM} \mathrm{NaCl}, 20 \mathrm{mM}$ imidazole) and lysis was performed with a mi- 
crofluidizer (2 cycles, 17,000 PSI). To purify inclusion bodies containing sCPY*, Triton $\mathrm{X}-100$ was added to a final concentration of $1 \%(\mathrm{w} / \mathrm{v})$ and the lysate was centrifuged at 12,000 rpm in an SS-34 rotor (Thermo Scientific). The supernatant was discarded and the pellet was resuspended in buffer $\mathrm{M}_{\mathrm{S}}$ containing $1 \%(\mathrm{w} / \mathrm{v})$ LDAO. After solubilization for $30 \mathrm{~min}$, the sample was centrifuged at 10,000 rpm in an SS-34 rotor for $30 \mathrm{~min}$. HisPur resin was added and the sample was nutated for $3 \mathrm{~h}$. The beads were washed with buffer $\mathrm{M}_{\mathrm{S}}$ containing $0.023 \%$ (w/v) LDAO and $150 \mathrm{mM} \mathrm{NaCl}$. The protein was eluted by overnight incubation of the beads with the SUMO protease Ulp1 $(1 \mu \mathrm{M})$. DTT $(1 \mathrm{mM})$ was added to the eluted sCPY* and the LDAO was exchanged to the detergent n-Nonyl $\beta$-D-glucopyranoside (NG) by anion exchange chromatography. Briefly, sCPY* was loaded onto a MonoQ 5/50 GL column (GE Healthcare) in buffer $\mathrm{S}_{\mathrm{X}}(20 \mathrm{mM}$ Tris- $\mathrm{HCl} \mathrm{pH}$ 7.4, $75 \mathrm{mM} \mathrm{NaCl}, 1 \mathrm{mM}$ DTT, $2 \% \mathrm{NG}$ ) and was eluted with a linear gradient up to $600 \mathrm{mM} \mathrm{NaCl}$ in $6 \mathrm{CVs}$. The protein was sortase-labeled and further purified on a Superdex 200 increase 10/300 GL column.

Ulp1: His 14 -TEV-Ulp1 was purified as follows. The cell pellet was resuspended in nickel wash containing $30 \mathrm{mM}$ imidazole. The cells were lysed in a microfluidizer (2 passages, 17,000 PSI) and the lysate was clarified by centrifugation at 40,000 rpm in a Ti45 rotor for $30 \mathrm{~min}$. HisPur resin $(8 \mathrm{ml})$ was added to the lysate and the sample was incubated for $3 \mathrm{~h}$ with rotation. The beads were washed with $50 \mathrm{CVs}$ of nickel wash and were eluted with buffer $\mathrm{I}_{\mathrm{U}}(20 \mathrm{mM}$ Tris-HCl pH 8.0, $500 \mathrm{mM} \mathrm{NaCl}, 500 \mathrm{mM}$ imidazole, $10 \%$ (w/v) glycerol, $4 \mathrm{mM}$ magnesium acetate). DTT (2 mM) was added to the elution fractions. The protein was either directly loaded onto a HiLoad Superdex 200 16/60 column equilibrated with buffer $\mathrm{L}_{\mathrm{U}}(20 \mathrm{mM}$ HEPES-KOH pH 7.4, $150 \mathrm{mM}$ $\mathrm{NaCl}, 10 \%$ (w/v) glycerol, $1 \mathrm{mM} \mathrm{DTT}$ ), or the His tag was removed. For His tag removal, the protein was dialyzed overnight against buffer $\mathrm{N}_{\mathrm{U}}(20 \mathrm{mM}$ HEPES-KOH $\mathrm{pH} 7.4,150 \mathrm{mM} \mathrm{NaCl}, 10 \%$ (w/v) glycerol, $15 \mathrm{mM}$ imidazole) in the presence of a 1:10 molar ratio of His-tagged TEV protease. The TEV protease was removed by reverse nickel-affinity chromatography and the flow-through was purified by gel filtration as described above.

Ubc6 and Ubc6-SBP: This protocol was kindly provided by Claudia C. Schmidt (Max Planck Institute for Biophysical Chemistry). After expression, the cell pellet was resuspended in nickel wash with $2 \mathrm{mM}$ EDTA and $2 \mathrm{mM}$ DTT. PMSF (1 mM) was added and the cells were lysed in a microfluidizer, as described above. After lysis, the unbroken cells were pelleted by centrifugation at 5,100 rpm in a F14-6x250y rotor for $10 \mathrm{~min}$. The supernatant was centrifuged at 42,000 rpm in a Ti45 rotor for $1 \mathrm{~h}$. The pellet was resuspended in nickel wash with $1 \%(\mathrm{w} / \mathrm{v})$ DDM and was solubilized for 1 
$\mathrm{h}$ with stirring. Insoluble material was removed by centrifugation at 42,000 rpm in a Ti45 rotor for $30 \mathrm{~min}$. HisPur resin $(6 \mathrm{ml})$ was added and incubated with the lysate for $2.5 \mathrm{~h}$. The beads were washed with nickel wash buffer containing $0.03 \%(\mathrm{w} / \mathrm{v})$ DDM and Ubc6 was eluted by cleavage with $0.5 \mu \mathrm{M}$ Ulp1 for $1 \mathrm{~h}$. Further purification was performed by gel filtration on a HiLoad Superdex 200 16/60 column equilibrated with buffer $\mathrm{L}_{\mathrm{U}}(20 \mathrm{mM}$ HEPES-KOH pH 7.5, $200 \mathrm{mM} \mathrm{NaCl}, 1 \mathrm{mM}$ DTT, 0.03\% (w/v) $\mathrm{DDM})$.

Doa10 RING domain: This protocol was kindly provided by Claudia C. Schmidt (Max Planck Institute for Biophysical Chemistry). The cell pellet was resuspended in nickel wash and lysis was performed as with Ubc6. The crude lysate was clarified by centrifugation at 42,000 $\mathrm{rpm}$ in a Ti45 rotor for $45 \mathrm{~min}$. HisPur resin $(6 \mathrm{ml})$ was added and was incubated with the lysate for $2.5 \mathrm{~h}$. The beads were washed with nickel wash buffer and eluted with nickel wash containing $500 \mathrm{mM}$ imidazole. DTT $(0.5 \mathrm{mM})$ was added and the protein was incubated with Ulp1 $(0.5 \mu \mathrm{M})$ to cleave off the His ${ }_{14}$-SUMO tag. The protein was dialyzed against buffer $\mathrm{D}_{\mathrm{R}}(20 \mathrm{mM}$ Tris- $\mathrm{HCl} \mathrm{pH}$ 7.5, $100 \mathrm{mM}$ $\mathrm{NaCl}$ ) and was purified further on a MonoQ column, followed by gel filtration using a Superdex 75 10/300 GL column (GE Healthcare).

ApoE 422K: Expression was induced with $0.5 \mathrm{mM}$ IPTG for $3 \mathrm{~h}$ at $25^{\circ} \mathrm{C}$. The cell pellet was resuspended in nickel wash and lysed as with Ubc6. The lysate was centrifuged at 42,000 rpm in a Ti45 rotor for $45 \mathrm{~min}$ and $8 \mathrm{ml}$ of HisPur resin was added to the supernatant. The sample was nutated for $2.5 \mathrm{~h}$ and the beads were washed with nickel wash buffer. The protein was eluted by incubation with $0.5 \mu \mathrm{M}$ Ulp1 for $1 \mathrm{~h}$. The eluted protein was dialyzed against buffer $\mathrm{D}_{\mathrm{R}}$, as with the Doa10 RING domain. Further purification was achieved by anion exchange chromatography on a MonoQ 10/100 GL column (GE Healthcare), eluting with a linear gradient of $0.1 \mathrm{M}$ to $1 \mathrm{M}$ $\mathrm{NaCl}$ over $15 \mathrm{CVs}$. The peak fractions were pooled and the protein was dialyzed against buffer $\mathrm{D}_{\mathrm{A}}(20 \mathrm{mM}$ HEPES-KOH pH 7.5, $150 \mathrm{mM} \mathrm{NaCl})$, followed by concentration in an Amicon $10 \mathrm{kDa}$ cutoff centrifugal filter.

Synaptobrevin and $\Delta N$ complex: Synaptobrevin was purified as previously described (Fasshauer et al., 1997; Stein et al., 2007), except that the detergent DM (final concentration $5 \mathrm{mM}$ ) was used instead of CHAPS. The $\Delta \mathrm{N} 49$ and 53 complex were purified as previously described (Stein et al., 2007), with the exception that $2 \%$ (w/v) OG and $200 \mathrm{mM}$ sucrose were used in place of CHAPS.

Uba1, Ubc'7 and Cue1-c: These proteins were purified as previously described (Stein et al., 2014). 
$C P Y^{*}-T M$ and $C P Y^{*}-T M 2:$ These proteins were purified as described in (Baldridge and Rapoport, 2016).

Doa10: Doa10, from S. cerevisiae, was kindly provided by Claudia C. Schmidt (Max Planck Institute for Biophysical Chemistry).

\subsection{Sortase labeling}

Sortase labeling of proteins with fluorescent dyes was performed as previously described (Stein et al., 2014), with a few modifications. A short peptide with the sequence GGGC (Thermo Scientific) was labeled with a maleimide-conjugated fluorescent dye (DyLight 680, DyLight 800 or Alexa Fluor 488, all from Thermo Scientific) in the following manner. The peptide was dissolved in $100 \mathrm{mM}$ HEPES-KOH pH 7.4, and a 1.5-molar excess was added to the lyophilized dye. The reaction was incubated in the dark for $1 \mathrm{~h}$ at RT and was quenched by adding $10 \mathrm{mM}$ DTT. The LPETGG-tagged protein was incubated with a 5-fold molar excess of the labeled peptide along with $15 \mu \mathrm{M}$ of the sortase A pentamutant (Chen et al., 2011) and $10 \mathrm{mM} \mathrm{CaCl}_{2}$. The reaction was incubated for $16 \mathrm{~h}$ at $4^{\circ} \mathrm{C}$, protected from light. The labeled protein was purified by sizeexclusion chromatography and was visualized by SDS-PAGE and fluorescence scanning in an Odyssey scanner (LiCOR) or an FLA 7000 scanner (Fujifilm). Labeling was determined by absorbance measurements, according to the manufacturer's instructions. The efficiency varied between 20-60\%, depending on the protein.

\subsection{SDS-PAGE and western blotting}

SDS-polyacrylamide gel electrophoresis (SDS-PAGE) was performed using Criterion TGX Stain-Free gels (4-20\% gradient gels) and the Criterion system (Bio-Rad), according to the manufacturer's instructions. The SDS sample buffer recipe is described in Schaegger (2006). Colloidal coomassie staining was performed as described (Dyballa and Metzger, 2009). Western blotting was performed using the Trans-Blot Turbo transfer system (Bio-Rad) for transfer onto nitrocellulose membranes, according to the manufacturer's instructions. The blots were blocked with $5 \%$ milk $(\mathrm{w} / \mathrm{v})$ in TBS buffer with $0.5 \%(\mathrm{v} / \mathrm{v})$ Tween-20 (TBS-T). The primary antibody (1:2500 dilution for mouse anti SBP, MAB10764 MilliPore) was diluted in 5\% milk TBS-T and incubated was for $1 \mathrm{~h}$ at RT. The blots were washed and incubated with DL680 or DL800-conjugated secondary antibodies (1:15000 dilution, LiCOR) in 5\% milk TBS-T for $1 \mathrm{~h}$ at RT. 
After washing, the blots were imaged by fluorescence scanning in an Odyssey scanner (LiCOR). Alternatively, the blot was incubated with an HRP-conjugated secondary antibody for $1 \mathrm{~h}$ at RT (1:2000 dilution, Bio-Rad), and was imaged using the ECL select western blotting detection kit (Perkin Elmer) and the LAS-1000 CCD detector (Fujifilm).

\subsection{Protein reconstitution into liposomes}

All protein reconstitutions into liposomes were performed using a method modified from Hernandez et al. (2012), which was modified from Rigaud and Lévy (2003). Preformed empty liposomes of $100 \mathrm{~nm}$ diameter (referred to as template from here on) were formed using the reverse-phase evaporation method described in Hernandez et al. (2012). The template contained a molar ratio of 6:2:1:1 POPC:DOPE:DOPS:Cholesterol, and the final lipid concentration was $20 \mathrm{mM}$. Proteoliposome reconstitutions were performed using liposome template as the starting lipid material. To this, detergent at a concentration termed the $\mathrm{R}$ value was added (Rigaud and Lévy, 2003), followed by the proteins to be reconstituted. The $\mathrm{R}$ value is defined by the following expression, from Rigaud and Lévy (2003):

$$
R=\frac{\left[D_{\text {total }}-D_{c m c}\right]}{[\text { lipid }]}
$$

where $D_{\text {total }}$ is the total detergent concentration and $D_{c m c}$ is the critical micellar concentration $(\mathrm{cmc})$ of the detergent. It was established that SNAREs reconstitute well at $R$ values of above 1 (Hernandez et al., 2012). Each membrane protein will have an optimal range of $\mathrm{R}$ values, which needs to be experimentally determined. In general, the greater the $\mathrm{R}$ value, the more complete the solubilization of the liposome template.

\subsubsection{Substrate encapsulation into liposomes using the NiNTA method}

To encapsulate substrates into liposomes, template was formed with $2 \%$ (mol\%) NiNTA lipids. $4 \mathrm{mM}$ of template liposomes were mixed with $\mathrm{OG}$ at an $\mathrm{R}$ value of 3 , along with $10 \mu \mathrm{M} \Delta \mathrm{N} 49$ complex and $1 \mu \mathrm{M}$ substrate, all in buffer L (20 mM HEPES-KOH pH 7.4, $150 \mathrm{mM} \mathrm{KCl}, 5 \mathrm{mM} \mathrm{MgAc}, 1 \mathrm{mM}$ TCEP). Additionally, urea at a final concentration of $1 \mathrm{M}$ was included. After incubation for $1 \mathrm{~h}$ at RT, the detergent was removed by overnight dialysis against buffer $\mathrm{L}$ in a $2 \mathrm{kDa}$ Slide-A-Lyzer cassette (Pierce). $1 \mathrm{~g}$ of 
Bio-Beads SM-2 (Bio-Rad) were added to each $300 \mathrm{ml}$ of buffer. Dialysis was performed at RT. To remove outside-bound substrate, the liposomes were floated in a Nycodenz step gradient containing $4 \mathrm{M}$ urea and $400 \mathrm{mM}$ imidazole in the bottom layers, and 1 $\mathrm{M}$ urea in the top layers (see section 2.7.4). The liposomes floated in fractions 1 and 2 , and were harvested from the gradient and analyzed by a protease protection assay (see section 2.7.5). In Figure 3.15, $1 \mu \mathrm{M} \mathrm{His}{ }_{14}$-Ulp1 was included in the encapsulation with and $2 \mu \mathrm{M} \Delta \mathrm{N} 49$. In Figure 3.19, $1 \mu \mathrm{M}$ His $_{14}$-Ulp1 was included in the Hrd1/Syb coreconstitution, with $2 \%$ NiNTA template (see section 2.7.2). To reconstitute Ubc6 with $\Delta$ N49 (Figure 3.14), the same protocol was performed as in the NiNTA method, but without NiNTA lipids. $2 \mu \mathrm{M}$ Ubc6 was added with an $\mathrm{R}$ value of 1.5 , and the liposome were not floated.

In Figures 3.3 and 3.4, encapsulation was performed without the use of NiNTA lipids. Briefly, $8 \mathrm{mM}$ of template was mixed with $\mathrm{OG}$ at an $\mathrm{R}$ value of 2.5 , along with $8 \mu \mathrm{M}$ Syb and $8 \mu \mathrm{M}$ sCPY*-A488, in buffer L. Detergent was removed by dialysis as above, with and without $2 \mathrm{M}$ urea. Liposomes were floated in a Nycodenz gradient and fraction 2 was harvested for protease protection assays. Direct reconstitution of Hrd1 into $C P Y^{*}$ liposomes: In Figure 3.25, Hrd1 was directly incorporated into liposomes containing encapsulated CPY*, modified from Baldridge and Rapoport (2016). CPY* was encapsulated as described above, and the floated liposomes were incubated with DMNG at an $\mathrm{R}$ value of 0.35 , for 30 min on ice. Lipid concentration of CPY* liposomes was estimated as approximately $2 \mathrm{mM}$, accounting for dilution after flotation. $1 \mu \mathrm{M}$ Hrd1 was added and the liposomes were incubated on ice for $1 \mathrm{~h}$. Detergent was removed with three sequential incubations in detergent removal spin columns (Pierce), with each incubation for $30 \mathrm{~min}$ at RT. The liposomes were analyzed by flotation in a Nycodenz gradient.

\subsubsection{Hrd1 coreconstitution with Syb into liposomes}

To coreconstitute Hrd1 with Syb, $4 \mathrm{mM}$ of template liposomes were mixed with DM at an $\mathrm{R}$ value of 1.5, along with $2 \mu \mathrm{M}$ Hrd1 and $10 \mu \mathrm{M}$ Syb, all in buffer L. Detergent was removed by sequential addition of detergent-removal spin column resin (referred to as SCR) (Pierce). Briefly, SCR was washed with buffer L, and 3 x $50 \mathrm{mg}$ were added per $200 \mathrm{\mu l}$ of liposome mix. Incubations were carried out for $20 \mathrm{~min}$ at RT. The liposome solution was centrifuged through a spin column at $1500 \mathrm{x}$ g after each incubation with SCR. Optimizations of Syb reconstitution without Hrd1 (Figure 3.9) were performed as above. $\mathrm{R}$ values of 1.5 were used for OG, DM and UDM. As a control, OG was removed 
by dialysis, as in section 3.9. When testing DMNG R values with Syb reconstitution (Figure 3.8), detergent was removed by application to two successive detergent removal spin columns (Pierce), according to manufacturer's instructions.

\subsubsection{Reconstitutions of Hrd1, Hrd1/Ubc6 and Ubc6 into liposomes}

When Hrd1 or Hrd1 mutants were reconstituted into liposomes alone or with Ubc6, DMNG was the solubilization detergent used. For Hrd1 reconstitution, $4 \mathrm{mM}$ template liposomes were mixed with DMNG at an $\mathrm{R}$ value of 1.5, along with $2 \mu \mathrm{M} \mathrm{Hrd1}$, in buffer $\mathrm{L}$ (see section 2.7.1). The mixture was incubated for $1 \mathrm{~h}$ at RT and detergent was removed by applying the solution to two consecutive detergent removal spin columns (Pierce). Each incubation was performed for $15 \mathrm{~min}$ at RT, and liposomes were eluted by centrifugation at $1200 \mathrm{x}$ g. A maximum volume of $150 \mathrm{\mu l}$ was added to each spin column. In Hrd1 reconstitution for planar lipid bilayer experiments, the protocol was the same with the exception that $8 \mathrm{mM}$ lipids and $8 \mu \mathrm{M}$ Hrd1 were used, with three consecutive spin columns for detergent removal instead of two. Hrd1 and Ubc6 were coreconstituted into liposomes in the following manner (Figure 3.38). $4 \mathrm{mM}$ template liposomes were mixed with DMNG at an $\mathrm{R}$ value of 1.5 , along with $2 \mu \mathrm{M}$ Hrd1 and $2 \mu \mathrm{M}$ Ubc6, in buffer L. After $1 \mathrm{~h}$ of incubation at RT, detergent was removed by incubating the solution in two consecutive detergent removal spin columns. The protocol for reconstituting Ubc6-SBP into liposomes (Figure 3.39) was kindly provided by Claudia C. Schmidt (Max Planck Institute for Biophysical Chemistry). Briefly, 4 $\mathrm{mM}$ of template was mixed with $\mathrm{OG}$ at an $\mathrm{R}$ value of 2 , along with $2 \mu \mathrm{M}$ Ubc6-SBP, in buffer L. The mixture was incubated for $1 \mathrm{~h}$ at RT and detergent was removed by three sequential incubations with SCR (10 mg, $30 \mathrm{mg}, 30 \mathrm{mg}$ of SCR per $100 \mathrm{\mu l}$ of liposomes). Each incubation with SCR was performed for $20 \mathrm{~min}$ at RT and the liposomes were collected by spin elution at $1200 \mathrm{x}$ g after each incubation.

\subsubsection{Nycodenz flotations}

Liposomes were floated in Nycodenz step gradients to assess protein incorporation and also as a purification technique to remove outside-bound substrate during encapsulation. Nycodenz stocks were prepared in buffer L. In scale flotations, $50 \mu \mathrm{l}$ of $80 \%$ (w/v) Nycodenz was mixed with $50 \mu \mathrm{l}$ of liposomes. This was overlaid with $40 \mu \mathrm{l}$ of $30 \%$, $15 \%$ and $0 \%$ Nycodenz solutions. In large scale flotations, $250 \mu \mathrm{l}$ of $80 \%$ Nycodenz was 
mixed with $250 \mu \mathrm{l}$ of liposomes, and this was overlaid with $500 \mu \mathrm{l}$ of $30 \%, 15 \%$ and 0\% Nycodenz each. The gradients were centrifuged at 50,000 rpm in an S55-S rotor (Thermo Scientific) for $1 \mathrm{~h}$ (small scale) and $2.5 \mathrm{~h}$ (large scale) flotations. Six equal fractions were harvested from the top of the gradient and were analyzed by SDS-PAGE.

\subsubsection{Protease protection assays}

Protease protections were performed to assess encapsulation efficiency and orientation of membrane proteins in liposomes. Encapsulation was judged by accessibility to proteinase $\mathrm{K}(\mathrm{PK})$. Briefly, $3 \mathrm{\mu l}$ of liposomes were incubated with $0.01 \mathrm{mg} / \mathrm{ml} \mathrm{PK}$ in a final volume of $15 \mathrm{\mu l}$ in buffer L. As a positive control, $0.67 \%$ (v/v) of Triton X-100 was included. The reaction was incubated for $20 \mathrm{~min}$ at RT and was quenched by addition of $0.5 \mu \mathrm{l}$ of a $200 \mathrm{mM}$ stock of PMSF (6.5 mM final concentration). The PMSF was incubated for $10 \mathrm{~min}$ and then SDS-sample buffer was added. The reactions were analyzed by SDS-PAGE and fluorescence scanning. Quantification was performed using ImageStudio Lite software (Li-COR). Protections with TEV protease with Hrd1 liposomes were performed as with PK protections, except $0.2 \mathrm{mg} / \mathrm{ml}$ TEV protease was added and reactions were incubated for $30 \mathrm{~min}$ at RT. As a positive control, 6 mM DMNG was included. Reactions were quenched by adding SDS-sample buffer. Protections with $3 \mathrm{C}$ protease were performed as protections with TEV protease, with a final concentration of $14 \mu \mathrm{M}$ of $3 \mathrm{C}$ protease.

\subsection{Hrd1 reconstitution into nanodiscs}

Hrd1 reconstitution into nanodiscs was modified from a previously-described protocol for SNARE incorporation into nanodiscs using the scaffold protein ApoE422K (Bello et al., 2016). In the initial optimizations of Hrd1 reconstitution into nanodiscs, POPC template liposomes with $100 \mathrm{~nm}$ diameter were used as the starting lipid material, which were prepared as previously described (Hernandez et al., 2012). The reasoning was that DMNG was poor in solubilizing lipids directly from a lipid film, whereas DMNG solubilization of liposome template was efficient. Briefly, POPC template liposomes (lipid concentrations indicated in the results section) were mixed with DMNG at an $\mathrm{R}$ value of 2.5, in buffer L. ApoE422K and Hrd1 were added at the indicated ApoE:Hrd1:POPC ratios. The mixture was incubated for $16 \mathrm{~h}$ at $4^{\circ} \mathrm{C}$ and detergent was removed by application of the mixture to three consecutive detergent removal spin columns, as in section 2.7.3, with incubation times of 30 min at RT per spin column. 
The nanodiscs were characterized by size exclusion chromatography on a Superose 6 5/15 GL column (GE Healthcare) in buffer L. It was later concluded that preparation of Hrd1 nanodiscs with DM and a lipid film was preferential. For this, POPC was dissolved in chloroform and the chloroform was removed with a rotary evaporator (Rotovapor R-124, Büchi) at 20 mbar. The lipid film was resuspended in buffer $\mathrm{N}_{\mathrm{S}}(20$ mM HEPES-KOH pH 7.4, $150 \mathrm{mM} \mathrm{NaCl}, 2 \%$ (w/v) DM) to a final lipid concentration of $10 \mathrm{mM}$. For reconstitution, $500 \mu \mathrm{l}$ of POPC/DM micelles were mixed with $10 \mu \mathrm{M}$ Hrd1 and $31.25 \mu \mathrm{M}$ ApoE422K in a total volume of $1 \mathrm{ml}$, in buffer L. The resulting ApoE:Hrd1:POPC ratio was 1:0.26:160. The mixture was incubated for $16 \mathrm{~h}$ at $4^{\circ} \mathrm{C}$ and detergent was removed by four sequential incubations with $200 \mathrm{mg}$ of SCR (see section 2.7.2), with each incubation lasting $20 \mathrm{~min}$ at RT. The solution was eluted through a spin column after each incubation step and transferred to a fresh tube. To further purify the nanodiscs, they were centrifuged through a 10-30\% (w/v) glycerol gradient, prepared in buffer N using a Gradient Master (BioComp Instruments). Centrifugation was performed for $20 \mathrm{~h}$ at $4^{\circ} \mathrm{C}$ in a SW 32 rotor (Beckman). 20 equal size fractions of approximately $1.5 \mathrm{ml}$ were collected from the top of the gradient and were analyzed by SDS-PAGE and coomassie staining. The peak Hrd1-containing fractions were pooled and concentrated in an Amicon $100 \mathrm{kDa}$ cutoff centrifugal filter, after which glycerol was removed by dialysis against buffer $\mathrm{N}_{\mathrm{D}}(20 \mathrm{mM}$ HEPES-KOH pH 7.4, $150 \mathrm{mM} \mathrm{NaCl}$ ). The Hrd1 concentration was determined by SDS-PAGE and colloidal coomassie staining (InstantBlue, Expedeon), using a Hrd1 standard curve. The gel was scanned using an Odyssey scanner (Li-COR) and quantification was performed using ImageStudio Lite software (Li-COR).

\subsubsection{Electron microscopy of Hrd1 nanodiscs}

Electron microscopy was performed by Dr. Dietmar Riedel, Max Planck Institute for Biophysical Chemistry. Briefly, the samples were applied to a glow-discharged carbon foil grid, washed with $0.1 \%$ glutaraldehyde, and stained with $1 \%$ uranyl acetate. Imaging was performed using a Talos L120C transmission electron microscope with a Ceta 16M camera (ThermoFisher- FEI, Netherlands). The size distribution of Hrd1 nanodiscs was calculated with iTEM software (Olympus Soft Imaging Solutions). 


\subsection{Fluorescence dequenching assay}

Membrane mixing was monitored with a fluorescence dequenching assay using NBD-PE and Rhodamine-PE, as previously described (Hernandez et al., 2012). Syb liposomes contained 1.5\% NBD-PE and 1.5\% Rhodamine-PE (molar percentage) and $\Delta$ N49 liposomes were unlabeled. $15 \mu \mathrm{l}$ of Syb liposomes were mixed with $20 \mu \mathrm{l}$ of $\Delta$ N49 liposomes in a total volume of $1200 \mathrm{\mu l}$, in buffer L. Reactions were measured in a quartz cuvette at $30^{\circ} \mathrm{C}$ and excitation and emission wavelengths were 460 and $538 \mathrm{~nm}$, respectively. In inhibition controls, $15 \mu \mathrm{M}$ of $\mathrm{Syb}_{\text {sol }}$ was pre-incubated with $\Delta \mathrm{N} 49$ liposomes, which contained $10 \mu \mathrm{M} \Delta \mathrm{N} 49$. After incubation for $5 \mathrm{~min}$ at RT, these liposomes were added to the fusion reaction.

\subsection{Fusion and ubiquitination assays}

$20 \mu \mathrm{l}$ of floated substrate liposomes were mixed with $15 \mu \mathrm{l}$ of Hrd1 liposomes in a total volume of $100 \mathrm{\mu l}$ in buffer $\mathrm{U}$ (20 mM HEPES-KOH pH 7.4, $150 \mathrm{mM} \mathrm{KCl,} 5 \mathrm{mM}$ $\mathrm{MgAc}, 0.5 \mathrm{mM}$ TCEP). The reaction was incubated for $45 \mathrm{~min}$ at $30^{\circ} \mathrm{C}$. In the inhibited fusion controls, $2 \mu \mathrm{M}$ Syb $_{\text {sol }}$ was mixed with substrate liposomes before adding Hrd1 liposomes. In reactions with unfloated substrate liposomes, $10 \mu \mathrm{l}$ were added instead of $20 \mathrm{\mu l} .40 \mathrm{\mu l}$ of fused liposomes were mixed with ubiquitination machinery in a total volume of $50 \mathrm{\mu l}$ in buffer $\mathrm{U}$. The final concentrations of the ubiquitination machinery were: $0.2 \mu \mathrm{M}$ Uba1, $2 \mu \mathrm{M}$ Ubc7, $2 \mu \mathrm{M}$ Cue1-c and $100 \mu \mathrm{M}$ ubiquitin. BSA $(0.2 \mathrm{mg} / \mathrm{ml})$ was included to reduce sticking to the tube. The reaction was started by adding $2.5 \mathrm{mM}$ ATP on ice. After taking the 0 min timepoint into SDS-sample buffer, the reactions were shifted to $30^{\circ} \mathrm{C}$ and timepoints were collected in SDS-sample buffer. Reactions were analyzed by SDS-PAGE and fluorescence scanning. The fusion assay with Hrd1 and Ubc6 liposomes was performed in the same manner, (Figure 3.14), except Ubc7 and Cue1-c were omitted from the ubiquitination mix.

\subsection{Ubiquitination assays with Hrd1 and substrates}

For Hrd1 ubiquitination assays in liposomes and nanodiscs, with or without cytosolic substrates, the following protocol was followed, modified from Stein et al. (2014). Ubiquitination reactions were performed in $50 \mu$ total volume, in buffer $\mathrm{U}$ (as in section 2.10), with $1 \mathrm{mg} / \mathrm{ml}$ BSA. The liposomes were diluted 1:10, so that the final Hrd1 
concentration was approximately $200 \mathrm{nM}$. Ubiquitination machinery was added with final concentrations as in section 2.10. Where indicated, substrates were added at 100 $\mathrm{nM}$ final concentration. Because the substrates were stored in urea, they were diluted into the reaction mixture immediately before starting the reaction. The reaction was pipetted on ice and $2.5 \mathrm{mM}$ ATP was added to start the reaction. The 0 min timepoint was taken into SDS-sample buffer and the reaction was shifted to $30^{\circ} \mathrm{C}$ in a thermocycler. Timepoints were taken into SDS-sample buffer and were analyzed by SDS-PAGE and fluorescence scanning. The ubiquitination of PrA* by Hrd1 in detergent (Figure 3.22) was performed as above, with $120 \mu \mathrm{M}$ DMNG instead of liposomes. The Hrd1 and $\mathrm{PrA}^{*}$ concentrations were $200 \mathrm{nM}$ each.

\subsection{Pulldown binding assays}

Binding assays with Hrd1 liposomes and Hrd1 nanodiscs were performed in the following manner, adapted from Stein et al. (2014). Hrd1 liposomes or nanodiscs were diluted to desired concentrations in buffer $\mathrm{B}_{\mathrm{B}}(20 \mathrm{mM}$ HEPES-KOH pH 7.4, $150 \mathrm{mM}$ potassium chloride, $5 \mathrm{mM}$ magnesium acetate, $0.5 \mathrm{mM}$ TCEP, $0.2 \mathrm{mg} / \mathrm{ml} \mathrm{BSA}$ ) and were immobilized onto streptavidin magnetic beads (Pierce), which were prewashed with buffer $\mathrm{B}_{\mathrm{A}}(20 \mathrm{mM}$ HEPES-KOH pH 7.4, $150 \mathrm{mM}$ potassium chloride, $5 \mathrm{mM}$ magnesium acetate, $0.5 \mathrm{mM}$ TCEP, $2 \mathrm{mg} / \mathrm{ml}$ BSA). Washing the beads with high concentrations of BSA was very important to prevent non-specific binding of substrates to the beads. Binding was performed for $1 \mathrm{~h}$ at RT and the beads were washed with buffer $\mathrm{B}_{\mathrm{B}}$. To verify binding of Hrd1, inputs and supernatants were analyzed by SDS-PAGE. Hrd1 binding to the beads was quantitative. $20 \mu \mathrm{l}$ of beads were used per $20 \mu \mathrm{l}$ of liposomes or nanodiscs. Ubiquitination was performed on beads by resuspending beads in ubiquitination mix, as described in section 2.10. Ubiquitination was performed for $1 \mathrm{~h}$ at $30^{\circ} \mathrm{C}$, and ATP was omitted in control reactions. The beads were washed with buffer $B_{A}$ and substrates (diluted freshly in buffer $B_{A}$ ) were incubated with the immobilized liposomes or nanodiscs for $30 \mathrm{~min}$ at RT. The supernatant containing the unbound fraction was collected and beads were washed with buffer $\mathrm{B}_{\mathrm{B}}$. The beads were eluted by incubation in buffer $B_{B}$ supplemented with $2 \mathrm{mM}$ biotin for $45 \mathrm{~min}$ at RT. In single concentration binding experiments, the inputs and supernatants were analyzed by SDS-PAGE and fluorescence scanning in an Odyssey scanner. In titration experiments, input and unbound fractions were analyzed by fluorescence scanning in a 384 well plate (Corning 3655) using the Odyssey scanner. The fluorescence was quantified using ImageStudio Lite software (Li-COR) and fraction bound was determined 
as 1-unbound fraction, normalizing to the unbound fraction in the beads only control. Fitting was performed using Origin 2018 software, with a one-site binding model, assuming 1:1 stoichiometry. PrA WT binding to Hrd1 liposomes was analyzed with SDS-PAGE and fluorescence scanning, followed by quantification using ImageStudio Lite software. Quantifications were from three binding experiments and error bars indicate standard deviation. Binding of CPY* to polyubiquitinated Ubc6-SBP in liposomes (Figure 3.39) was performed as above, with the exception that the ubiquitination mix was supplemented with $10 \mu \mathrm{M}$ of the Doa10 RING domain.

\subsection{Deubiquitination assays}

Deubiquitination of Hrd1 in liposomes (Figure 3.32C) was performed after purifying ubiquitinated Hrd1 liposomes on streptavidin magnetic beads. Briefly, Hrd1 liposomes were diluted to $0.5 \mu \mathrm{M}$ Hrd1 in buffer $\mathrm{B}_{\mathrm{B}}$ and were immobilized on prewashed streptavidin magnetic beads. The liposomes were incubated with the standard ubiquitination machinery (as in section 2.10) for $1 \mathrm{~h}$ at $30^{\circ} \mathrm{C}$. The beads were washed with buffer $\mathrm{B}_{\mathrm{B}}$ to remove the ubiquitination machinery and ubiquitinated Hrd1 was eluted with buffer $\mathrm{B}_{\mathrm{B}}$ supplemented with $2 \mathrm{mM}$ biotin. Usp2 was added at increasing concentrations and timepoints were taken into SDS-sample buffer. The reactions were analyzed by SDS-PAGE and fluorescence scanning. To quantify the fraction of released CPY* from Hrd1 after deubiquitination (Figure 3.40), Hrd1 liposomes (250 nM Hrd1) were immobilized onto streptavidin magnetic beads as described in section 2.12. $\mathrm{CPY}^{*}$ (50 $\mathrm{nM}$ ) was incubated for $30 \mathrm{~min}$ at RT and the unbound fraction was collected. The beads were washed and incubated with $2 \mu \mathrm{M}$ Usp2 (or with buffer) for $30 \mathrm{~min}$ at RT. The supernatants containing released CPY* were collected. The beads were washed and eluted with $2 \mathrm{mM}$ biotin in buffer $\mathrm{B}_{\mathrm{B}}$. The input, unbound, supernatants and elutions were analyzed by SDS PAGE and fluorescence scanning. To determine the fraction of $\mathrm{CPY}^{*}$ released, the elution fractions were quantified. CPY* release without Usp2 was normalized to 1. Quantification was performed from three experiments and error bars indicate standard deviation. 


\subsection{Electrophysiological experiments in planar lipid bilayers}

Electrophysiological measurements of Hrd1 using the planar lipid bilayer technique were performed by Dr. Niels Denkert (University Medical Center Göttingen and Max Planck Institute for Biophysical Chemistry), in the lab of Prof. Michael Meinecke (University Medical Center Göttingen). Further details about the technique are provided in section 3.8 and in Harsman et al. (2011). Experiments were performed as previously described (Denkert et al., 2017; Reinhold et al., 2012; Harsman et al., 2011), with all quantifications derived from three independent experiments, except where indicated. Error bars indicate standard error of the mean. To characterize channel activity, Hrd1 liposomes were incubated with ubiquitination machinery at $30^{\circ} \mathrm{C}$ for $1 \mathrm{~h}$, and were then added to the cis side of the chamber. In fusion conditions, the buffer contained 250 $\mathrm{mM} \mathrm{KCl}, 10 \mathrm{mM}$ MOPS pH 7.0 on the cis side and $20 \mathrm{mM} \mathrm{KCl}, 10 \mathrm{mM} \mathrm{MOPS} \mathrm{pH}$ 7.0 on the trans side. Upon fusion, the chambers were perfused with buffer containing $250 \mathrm{mM} \mathrm{KCl}$ on both sides of the membrane. Current traces were recorded at different holding potentials in symmetrical buffer conditions. The conductance histograms show conductance states from constant voltage conditions. To calculate channel diameter, the channel was treated as a cylindrical pore with a restriction zone of $2 \mathrm{~nm}$, assuming a fivefold greater resistivity than the resistivity of the bulk buffer, as previously prescribed (Smart et al., 1997). In substrate activation experiments, 100 nM CPY* was added to the indicated sides of the chamber after incorporation of Hrd1 channels. The solution was mixed with a magnetic stirrer for $2 \mathrm{~min}$, let to rest for $2 \mathrm{~min}$, and then recordings were continued. Fusion rates of Hrd1 channels were calculated by measuring the amount of fusions to the PLB over a $20 \mathrm{~h}$ time period. This was repeated three times, for a total of $60 \mathrm{~h}$ per condition. For calculation of channel lifetimes, the time between fusion of Hrd1 channels to the PLB and spontaneous channel closure was recorded. 


\section{Results}

\subsection{Developing a fusion system to study retrotranslocation by Hrd1}

\subsubsection{Overview}

Two previous Hrd1 reconstitutions paved the way for investigating mechanistic details of Hrd1-mediated retrotranslocation (Stein et al., 2014; Baldridge and Rapoport, 2016). However, these reconstitutions had limitations which precluded the study of direct retrotranslocation by Hrd1. In the reconstitution by Stein and colleagues, Hrd1 and the model substrate $\mathrm{CPY}^{*}$ were either reconstituted in detergent or coreconstituted as a preformed complex in liposomes. Importantly, $\mathrm{CPY}^{*}$ was not protected from outside proteases, indicating that it was already exposed to the outside of the liposomes. In the study by Baldridge et al, a membrane-anchored version of $\mathrm{CPY}^{*}$ was coreconstituted into liposomes and then Hrd1 was added to the same population of liposomes using detergent (Figure 1.8). This coreconstitution method leaves open the possibility that detergent-mediated complexes of Hrd1 and $\mathrm{CPY}^{*}$ were formed, which may represent intermediate states of retrotranslocation or non-native, detergent-mediated complexes. In order to keep Hrd1 and substrate separate before the retrotranslocation reaction, they must not be allowed to interact during the reconstitution. The fusion system solves this problem by reconstituting Hrd1 and $\mathrm{CPY}^{*}$ in separate populations of liposomes and bringing them together by SNARE-mediated fusion (Figure 3.1). In this manner, the substrate is delivered to the luminal side of Hrd1 in a detergent-free manner. The resulting fused liposomes are primed for a retrotranslocation reaction. Since the substrate is encapsulated in the lumen of the liposomes, subsequent movement or exposure of the substrate to the outside indicates retrotranslocation by Hrd1.

As Hrd1 autoubiquitination has been postulated to prime or open the channel (Baldridge and Rapoport, 2016), I initiated retrotranslocation by adding ubiquitination machinery and ATP. Because the model substrates $\mathrm{CPY}^{*}$ and $\mathrm{sCPY}^{*}$ are efficiently ubiquitinated 
by Hrd1 (Stein et al., 2014; Baldridge and Rapoport, 2016), the substrate should become ubiquitinated as it emerges from the lumen (Figure 3.1). As the encapsulated substrate is not accessible to the Hrd1 RING domain and ubiquitination machinery in the lumen of the liposomes, it can only be ubiquitinated if it moves through the membrane. Therefore, I analyzed substrate ubiquitination status as a readout for retrotranslocation efficiency.

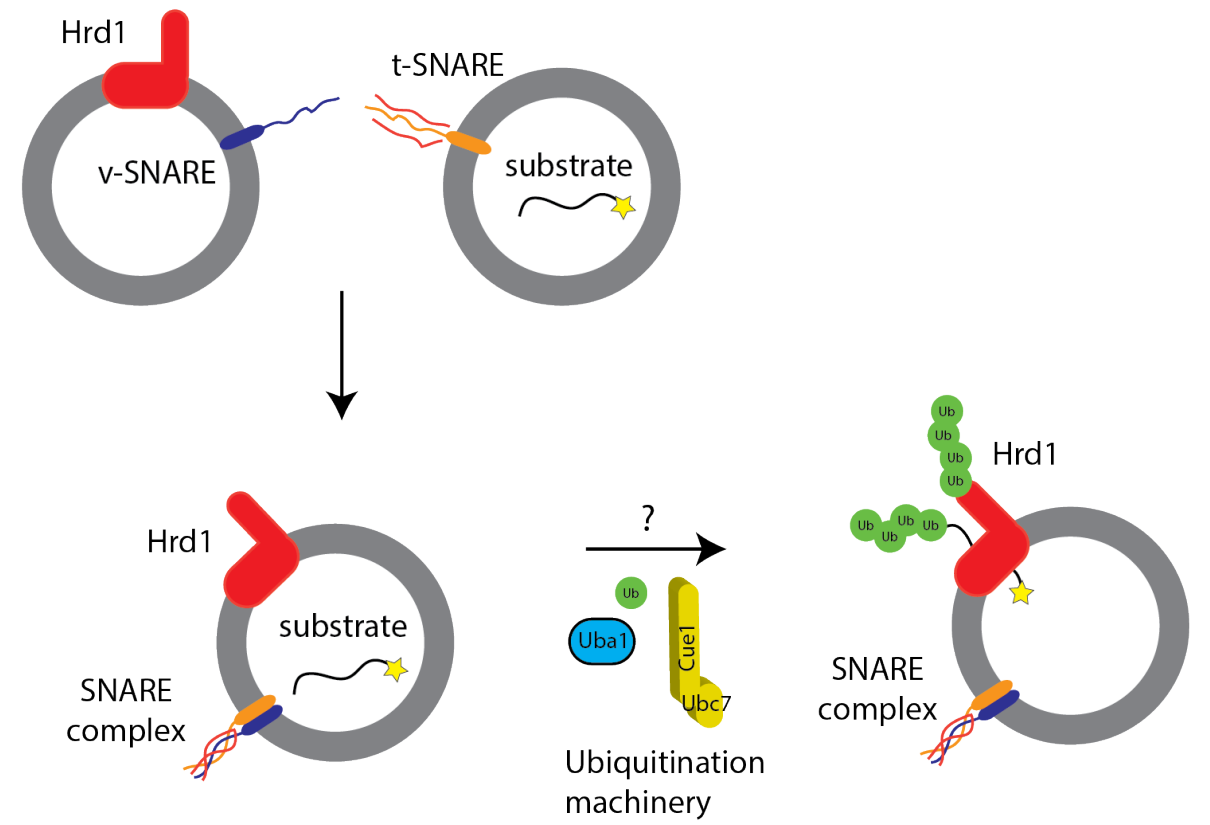

Figure 3.1: Overview of the fusion system

Hrd1 and an ERAD-L substrate are coreconstituted separately into liposomes and are brought together by SNARE-mediated fusion. Upon addition of ubiquitination machinery, Hrd1 autoubiquitinates, which is hypothesized to open the channel. Retrotranslocated substrate is ubiquitinated by Hrd1, which is monitored as a readout for retrotranslocation.

\subsection{Encapsulation of ERAD-L substrates}

In order to study retrotranslocation of a substrate by Hrd1, the substrate must first be encapsulated into the lumen of liposomes. The following section details the optimization of substrate encapsulation into liposomes.

\subsubsection{Substrate encapsulation using the traditional method}

One of the most challenging aspects of the fusion system is the encapsulation of a misfolded protein into the lumen of liposomes. Encapsulation of proteins into lipo- 
somes is not a well-established technique, but encapsulation of small molecules such as fluorescent dyes and oligonucleotides has been demonstrated (Nickel et al., 1999; Liu et al., 2017). The traditional method used to encapsulate a molecule into liposomes is to provide a high concentration of the molecule during liposome formation. In this manner, some of the molecules become entrapped in the lumen during bilayer formation. Thereafter, the non-encapsulated molecule is removed by various methods such as size exclusion chromatography, flotation, centrifugation or dialysis (Figure 3.2).

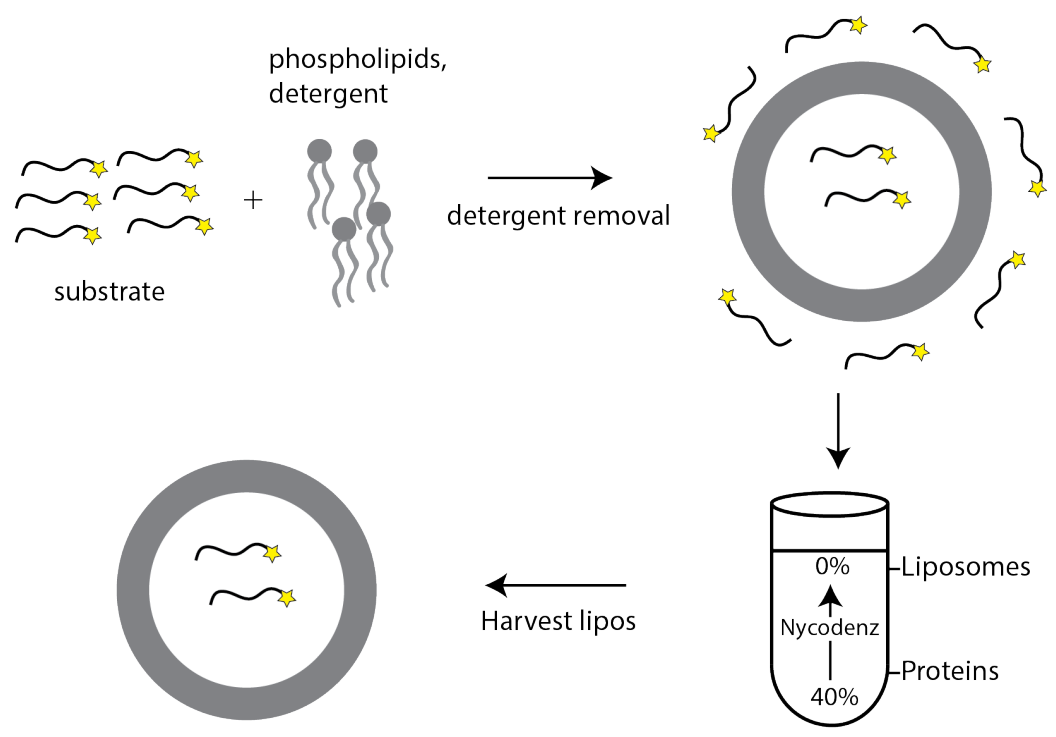

Figure 3.2: General strategy for encapsulation of a molecule into liposomes An excess of substrate is provided during detergent solubilization of lipids. Upon detergent removal and bilayer formation, a fraction of the substrate becomes entrapped in the lumen of the liposomes. Removal of the non-encapsulated substrate is achieved by further purification of the liposomes (in this case, by flotation in a density gradient).

I first attempted to apply this traditional method to encapsulate the model substrate sCPY*. To this end, sCPY* labeled with AlexaFluor 488 (sCPY*-488) was mixed with performed liposomes, the detergent OG (R-value 2.5) and the v-SNARE synaptobrevin, labeled with AlexaFluor 633 (Syb-633). Upon detergent removal by dialysis, the liposomes were floated in a nycodenz gradient with or without $2 \mathrm{M}$ urea (Figure 3.3). The presence of urea in the flotation helped to wash off some of the outside-bound sCPY* (compare lanes 4, 5 and 6 in no urea vs. 2M urea samples). However, Syb was also found in the bottom fractions after flotation in $2 \mathrm{M}$ urea, indicating that Syb was 
not properly reconstituted.

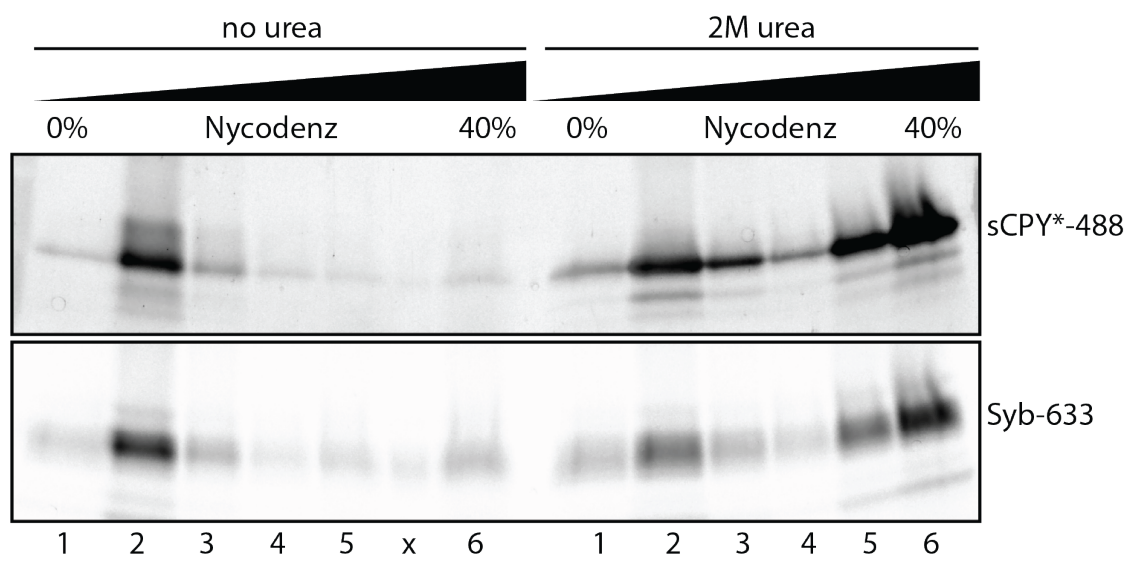

Figure 3.3: Flotation of sCPY*/Syb liposomes

Liposomes containing reconstituted $\mathrm{sCPY}^{*}$ and Syb were subjected to flotation in a Nycodenz gradient with or without $2 \mathrm{M}$ urea in the bottom layer of the gradient. Six fractions were collected from the top of the gradient and were analyzed by SDS-PAGE and fluorescence scanning. The $\mathrm{x}$ denotes a skipped lane.

To test for encapsulation of sCPY*, the floated liposomes were harvested (fraction 2) and a protease protection was performed with trypsin and proteinase $\mathrm{K}$. All of the sCPY* was cleaved by proteinase $\mathrm{K}$ and trypsin, and therefore neither sample showed any encapsulated sCPY* (Figure 3.4).

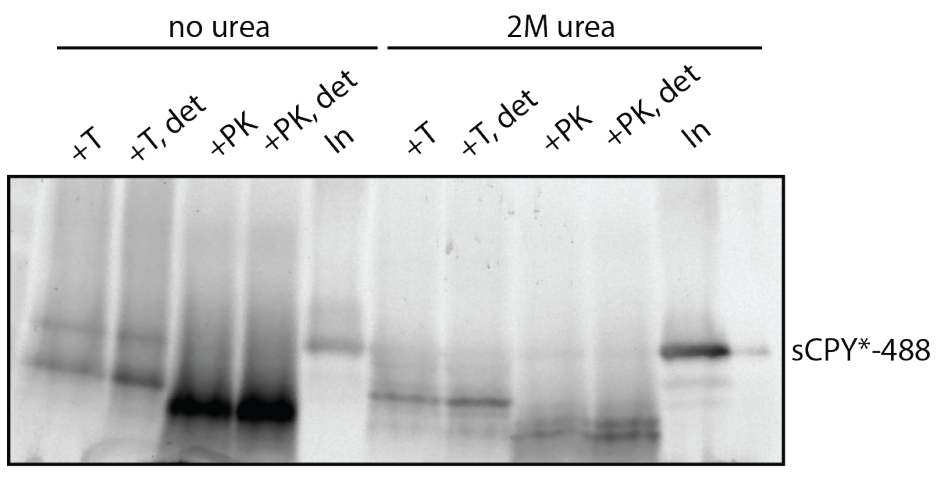

Figure 3.4: Protease protection of floated sCPY*/Syb liposomes

Floated liposomes were treated with trypsin (T) or proteinase K (PK) with or without DMNG (det). Samples were analyzed by SDS-PAGE and fluorescence scanning. In: Input.

Encapsulation of sCPY* using the traditional method proved to be challenging for multiple reasons. First, the model substrate sCPY* is misfolded and therefore aggregation- 
prone. To avoid aggregation, it needs to be purified in detergent or denaturing agents such as urea. When detergent and/or urea are removed during reconstitution, the substrate needs to be present at low concentrations to avoid aggregation. As encapsulation efficiency depends on the starting concentration of the substrate, this makes encapsulation using the traditional method inefficient.

\subsubsection{Substrate encapsulation using the NiNTA method}

In order to improve the efficiency of substrate encapsulation, I modified the traditional method by introducing an affinity step. The purpose of the affinity step is to concentrate the substrate onto the phospholipids, thereby increasing the likelihood of encapsulation during lipid bilayer formation. To this end, I made preformed liposomes with phospholipids labeled on their head group with NiNTA, which have been shown to significantly increase the encapsulation efficiency of a His-tagged protein (Colletier et al., 2002). His ${ }_{14}$-CPY*, phospholipids and $\triangle$ N49, a t-SNARE complex engineered for efficient in vitro fusion (Pobbati et al., 2006), were mixed with OG with an $\mathrm{R}$ value of 3. Detergent was removed by dialysis and outside-bound CPY* was removed by flotation in a Nycodenz gradient containing imidazole and urea in the bottom layers (Figure 3.5). Urea was included in the flotation because the $\mathrm{CPY}^{*}$ aggregates when detergent is removed and becomes otherwise insensitive to imidazole (data not shown). The OG-dialysis protocol was modified from a previously-established protocol for reconstitution of SNARE proteins (Hernandez et al., 2012). 


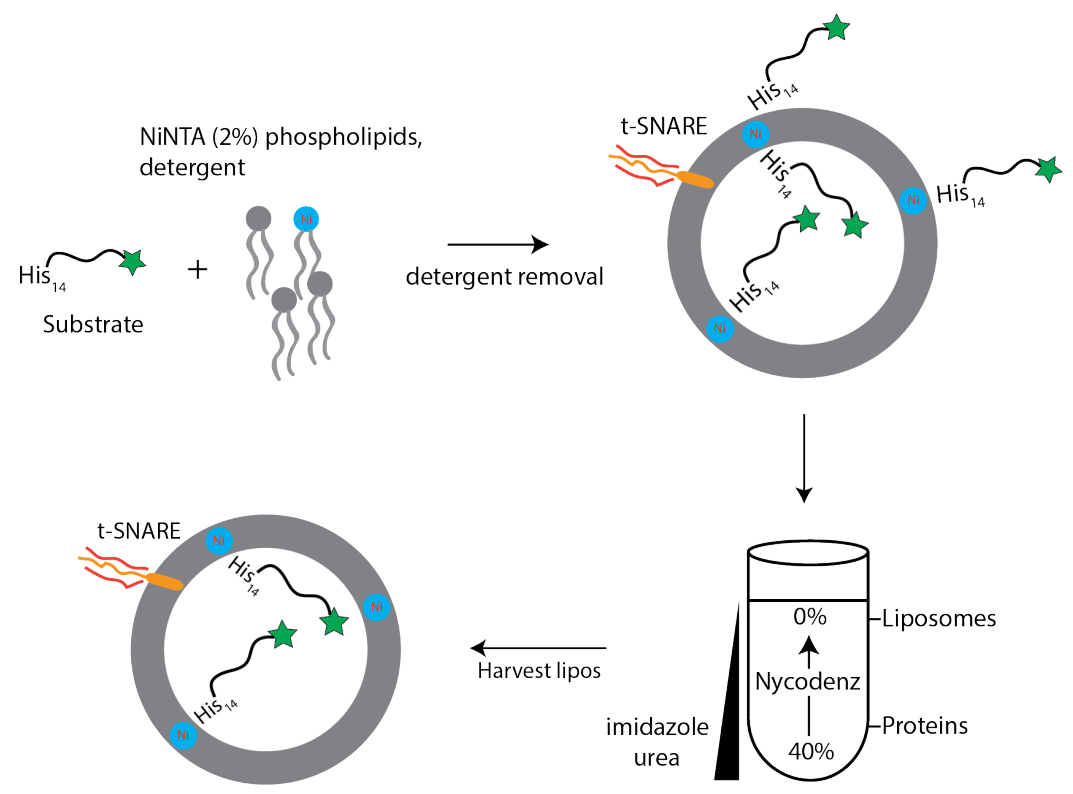

Figure 3.5: Strategy of substrate encapsulation using the NiNTA affinity method

A His-tagged substrate is solubilized in detergent with NiNTA-labeled phospholipids. Upon detergent removal, the substrate is bound to NiNTA-phospholipids to both sides of the membrane. Subsequent flotation in a Nycodenz gradient with imidazole and urea removes the outside-bound substrate.

Overall encapsulation efficiency was first tested by subjecting the unfloated liposomes to a protease protection assay (Figure 3.6). Increasing concentrations of urea were included during the solubilization and detergent removal steps in order to increase the encapsulation efficiency. Urea at concentrations of $1 \mathrm{M}$ and above significantly improved the encapsulation efficiency of $\mathrm{CPY}^{*}$, presumably by preventing aggregation during the detergent removal process (Figure 3.6). Therefore, at least 1M urea was included in all subsequent substrate encapsulations. The encapsulation efficiency with $2 \mathrm{M}$ urea was $30 \%$. 


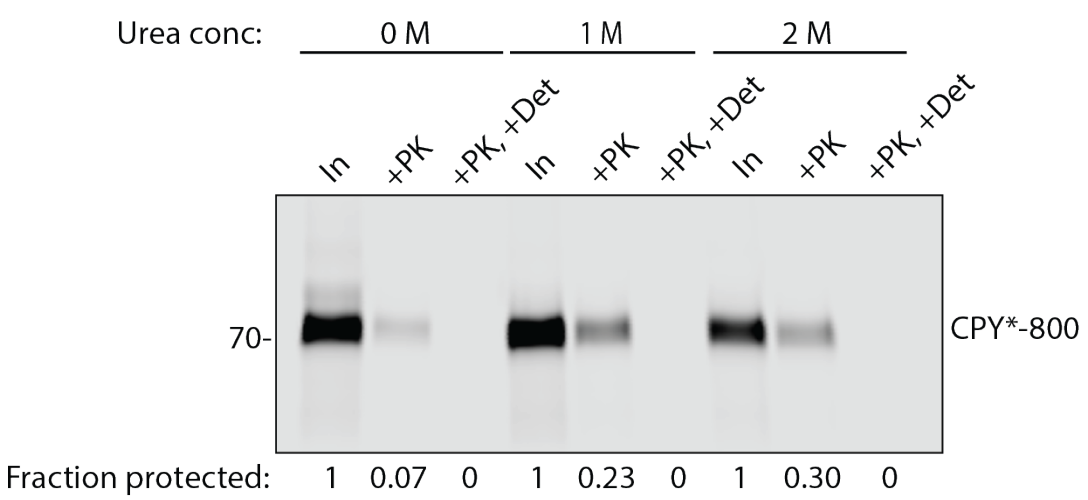

Figure 3.6: Encapsulation efficiency of $\mathrm{His}_{14}-\mathrm{CPY}^{*}$ with increasing concentrations of urea

His $_{14}$ CPY* $^{*}$ labeled with DL800 was reconstituted into liposomes containing 2\% NiNTA. Increasing concentrations of urea were included during the solubilization and detergent removal. The liposomes were subjected to protease protection with proteinase K (PK), with or without Triton X-100 (Det). The samples were analyzed by SDS-PAGE and fluorescence scanning.

$\mathrm{CPY}^{*} / \Delta \mathrm{N} 49$ liposomes from the $1 \mathrm{M}$ urea reconstitution were floated in a Nycodenz gradient with imidazole. After flotation, $\mathrm{CPY}^{*}$ was localized both in the top of the gradient as well as in the bottom fractions (Figure 3.7A). The CPY* in the bottom of the gradient represents the fraction that was washed off. Notably, the $\Delta$ N49 remained in the top fractions, indicating that it was well reconstituted, and demonstrating that this encapsulation method is compatible with SNARE reconstitution. The floated liposomes were harvested and encapsulation efficiency was analyzed by a protease protection assay. About $85 \%$ of the $\mathrm{CPY}^{*}$ was protected from digestion by proteinase $\mathrm{K}$ (Figure 3.7B). 
A

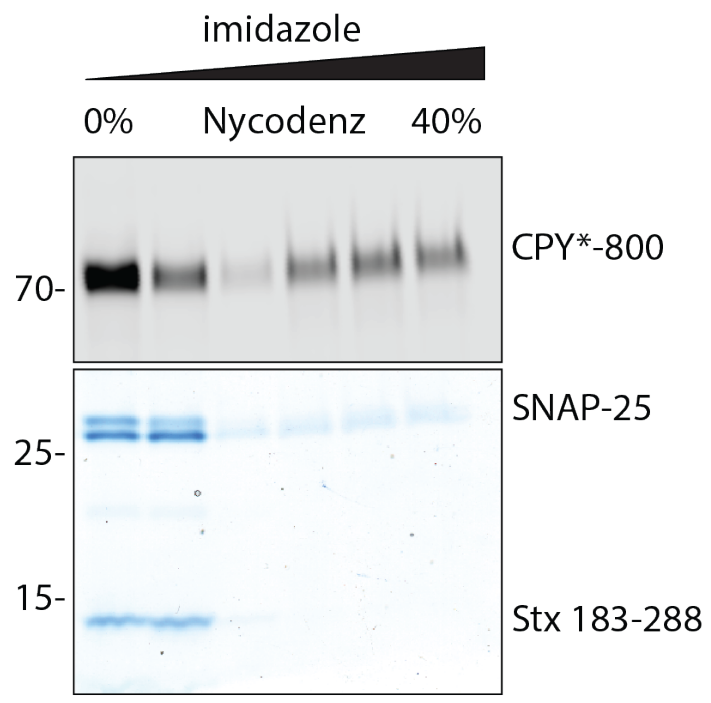

B

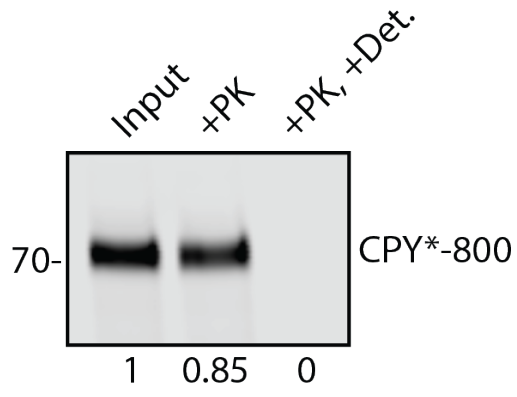

Figure 3.7: Imidazole flotation and protease protection of $\mathrm{His}_{14}$ $\mathrm{CPY}^{*} / \Delta \mathrm{N} 49$ liposomes

A: His $_{14}$ CPY $^{*} / \Delta$ N49 liposomes were subjected to flotation in a Nycodenz step gradient containing $400 \mathrm{mM}$ imidazole and $4 \mathrm{M}$ urea in the bottom layers. Six fractions were collected from the top of the gradient (left to right on gel) and were analyzed by SDS-PAGE, fluorescence scanning (top) and coomassie staining (bottom). SNAP-25 and Stx 183-288 are components of the $\Delta \mathrm{N} 49$ complex. B: The top two fractions were harvested from the gradient and were analyzed by protease protection with proteinase K (PK). Det: Triton X-100. Numbers below the lanes indicate the fraction protected from PK.

In the retrotranslocation assay, it is important to have most of the substrate encapsulated in the liposomal lumen because any outside-bound substrate contributes to background signal in the ubiquitination assay. Having an encapsulated fraction of about $85 \%$ was an acceptable starting point for the retrotranslocation assay. Although it would have been preferential to have close to $100 \%$ of the substrate encapsulated, some fraction of the outside-bound substrate was invariably resistant to imidazole and urea treatment.

\subsection{Coreconstitution of Hrd1 and Synaptobrevin}

\subsubsection{Optimization of reconstitution conditions for Hrd1 and Synaptobrevin}

In order to fuse substrate liposomes with Hrd1 liposomes, Hrd1 needed to be reconstituted with synaptobrevin (Syb), the v-SNARE complementary to the $\Delta$ N49 complex 
used in the substrate liposomes (Pobbati et al., 2006). In principle, the SNAREs are interchangeable, that is, it does not matter if Syb is reconstituted in the substrate liposomes or in the Hrd1 liposomes. However, practical limitations made it simpler to reconstitute Syb with Hrd1 instead of $\Delta \mathrm{N} 49$. Hrd1 is stable in only a few select detergents (Stein et al., 2014; Baldridge and Rapoport, 2016), whereas the activity of $\Delta \mathrm{N} 49$ is greatest when reconstituted with the OG-dialysis method (Hernandez et al., 2012) (Figure 3.9). Importantly, Hrd1 cannot be reconstituted with the OG-dialysis method because OG interferes with Hrd1 autoubiquitination activity (data not shown). I attempted to reconstitute $\Delta \mathrm{N} 49$ with the detergents compatible with Hrd1 reconstitution, but this resulted in poor fusion efficiency (data not shown). Syb is amenable to a wide variety of detergents and reconstitution conditions (personal observation), making it the better option for coreconstitution with Hrd1. When developing the reconstitution protocol for Hrd1 and Syb, I considered two main factors. First, the solubilization detergent had to be compatible with Hrd1. Second, the chosen detergent had to preserve fusion efficiency.

To screen for reconstitution conditions, I used an established lipid mixing assay as a readout for fusion (Struck et al., 1981; Hernandez et al., 2012) (see materials and methods). Upon fusion, unlabeled lipids from one population of liposomes mix with and dilute the NBD/Rhodamine FRET pair in another population of liposomes. Dequenching of the NBD FRET donor is monitored as the readout for fusion. Based on previous studies, I defined an efficient fusion reaction as a reaction having a relative two-fold increase in donor fluorescence in the first 5-10 min (Hernandez et al., 2012).

I first tested how the Hrd1-compatible detergent DMNG influenced Syb reconstitution (Stein et al., 2014). To this end, I made $\Delta$ N49 liposomes with the OG-dialysis method (as described in Hernandez et al. (2012)) and tested fusion to liposomes where Syb was reconstituted with either the OG-dialysis method (as described in Hernandez et al. (2012)), or with the detergent DMNG at a range of different $\mathrm{R}$ values (Figure 3.8). I checked whether I could determine an $\mathrm{R}$ value where the fusion rate was similar to the OG-dialysis method. As evident in Figure 3.8, DMNG-mediated reconstitution of Syb significantly inhibited fusion (compare the black trace to all other traces). At very low $\mathrm{R}$ values, fusion was nonexistent and matched the inhibited OG-dialysis reaction, where soluble Syb $\left(\mathrm{Syb}_{\mathrm{sol}}\right)$ to was added to $\Delta \mathrm{N} 49$ liposomes before fusion. There was some fusion activity between $\mathrm{R}$ values of 0.35 and 1 , but significantly less than the OG-dialysis control. Above $\mathrm{R}$ values of 1 , fusion was completely inhibited. Therefore, I concluded that DMNG was incompatible with Syb reconstitution. 


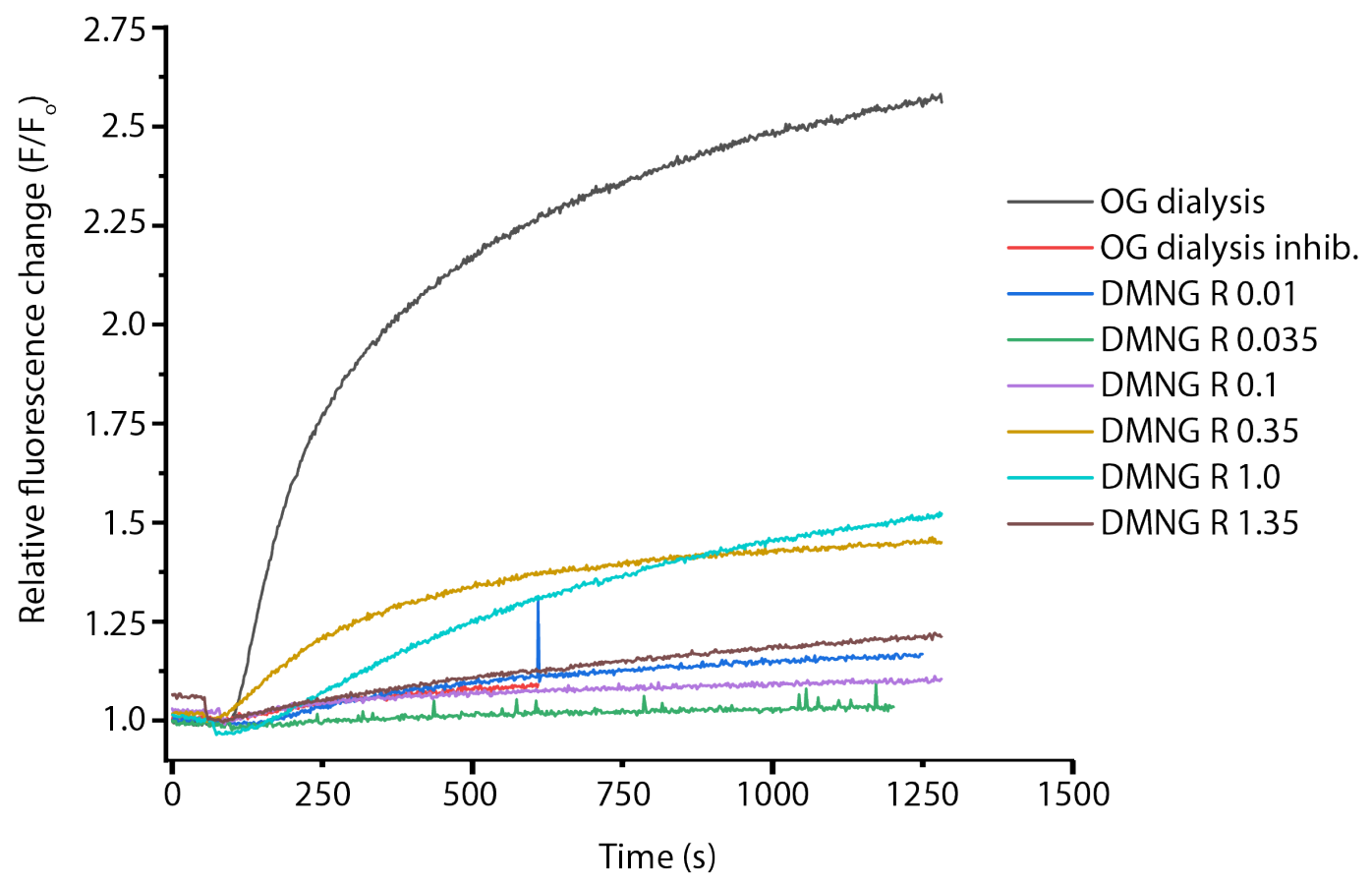

Figure 3.8: Effect of DMNG on Syb reconstitution and fusion

$\Delta \mathrm{N} 49$ was reconstituted in unlabeled liposomes using the OG-dialysis method and was fused with Syb reconstituted in NBD/Rh liposomes. Fusion was monitored as a function of the change in NBD fluorescence. Syb was reconstituted using OG-dialysis (black, red) or different $R$ values of DMNG, as indicated. Inhib: fusion was inhibited by incubating $\Delta \mathrm{N} 49$ liposomes with the soluble domain of $\mathrm{Syb}\left(\mathrm{Syb}_{\text {sol }}\right)$ prior to addition of Syb liposomes.

I next screened for detergents in Syb reconstitution that would result in better fusion efficiency than DMNG. I tested the detergents OG, DM, UDM and DDM. The detergents were removed with a cyclodextrin-based resin (SCR), which has a high affinity for a wide range of detergents (Antharavally et al., 2011). The rationale behind this removal method is that low-CMC detergents like DM, DDM and UDM are not efficiently removed by dialysis. This is especially important during Hrd1 reconstitution because trace amounts of the low-CMC detergent DMNG are introduced. Of all detergents tested, DM resulted in the best fusion efficiency compared to the OG-dialysis method (Figure 3.9, blue vs black traces). OG removed with SCR was the worst, while UDM had an intermediate effect on fusion efficiency (Figure 3.9, red and green traces). DDM-mediated Syb reconstitution was not viable because the lipids precipitated. Reconstitution of $\Delta \mathrm{N} 49$ with $\mathrm{OG}$ removed by SCR significantly decreased fusion efficiency compared to OG removal by dialysis (Figure 3.9, yellow vs black traces), indicating that $\Delta \mathrm{N} 49$ activity is sensitive to the detergent removal method. As SCR removes detergent more rapidly than dialysis, it appears that slow detergent removal favors 
proper $\Delta \mathrm{N} 49$ reconstitution. Overall, I concluded that DM was the best detergent for Syb reconstitution apart from OG-dialysis.

I then tested whether fusion efficiency was affected in the DM reconstitution when Hrd1 was coreconstituted. As seen in Figure 3.9, Hrd1/Syb coreconstitution slightly reduced fusion efficiency compared to Syb DM liposomes (cyan vs blue traces), but the overall efficiency was similar. Fusion efficiency was not affected when an additional tailanchored membrane protein, Ubc6, was coreconstituted in the $\Delta \mathrm{N}$ liposomes (Figure 3.9, brown vs cyan traces). Therefore, Hrd1/Syb coreconstitution using DM and SCR results in efficiently-fusing liposomes.

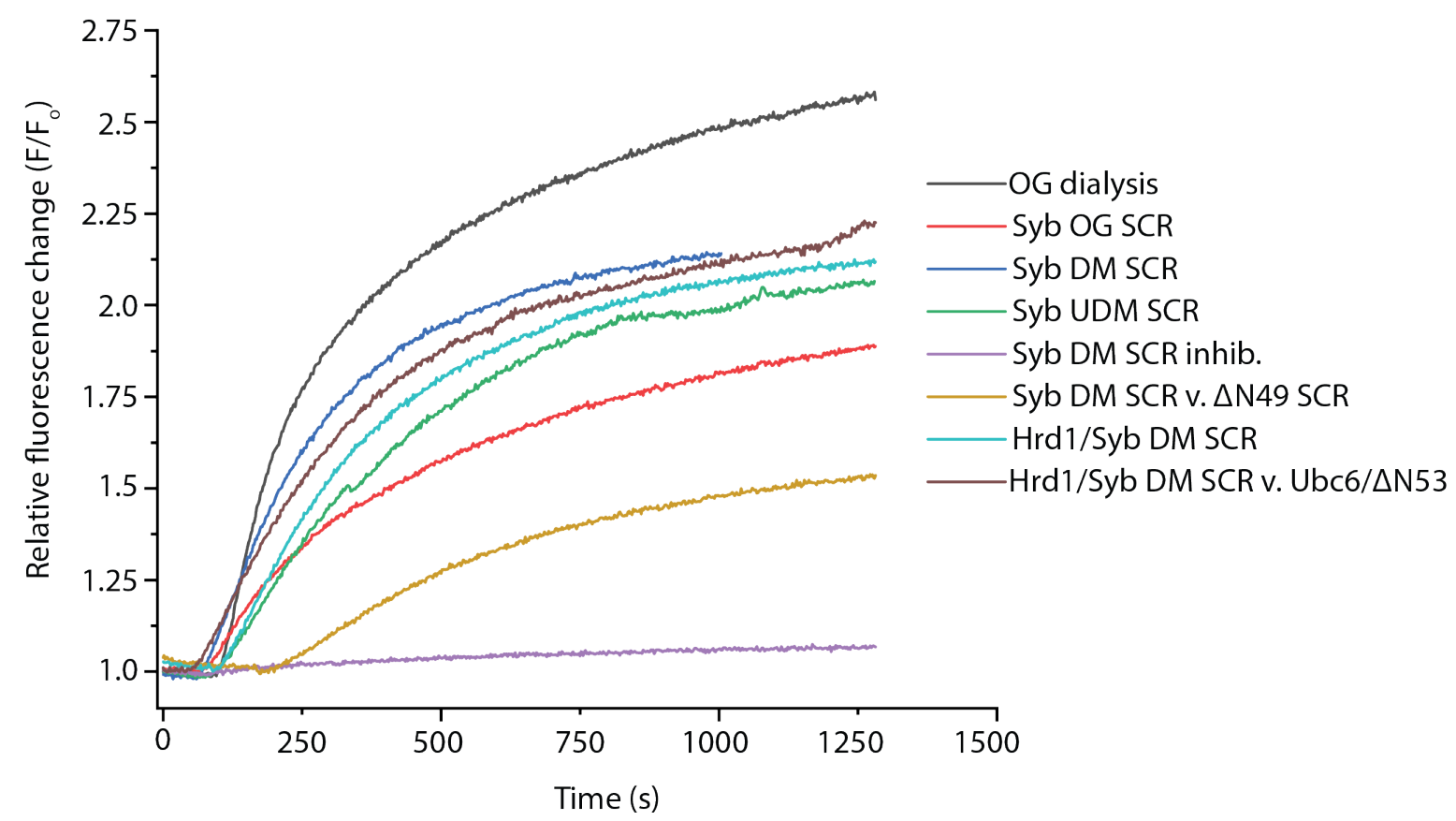

Figure 3.9: Effect of various detergents on SNARE and Hrd1 reconstitution Syb or Hrd1/Syb were reconstituted into NBD/Rh liposomes using the indicated detergents, and were fused with unlabeled lipsomes containing $\Delta \mathrm{N} 49$, prepared by OGdialysis. Fusion was monitored as a function of the change in NBD fluorescence. SCR indicates that detergent was removed with a cyclodextrin-based resin. Black trace: both Syb and $\Delta$ N49 were reconstituted with the OG-dialysis method. Inhib: fusion was inhibited by incubating $\Delta$ N49 liposomes with $\mathrm{Syb}_{\text {sol }} . \Delta \mathrm{N} 53$ is similar to $\Delta \mathrm{N} 49$, except the stabilizing Syb fragment contains residues 53-96 instead of 49-96 (Hernandez et al., 2012).

\subsubsection{Biochemical characterization of Hrd1/Syb liposomes}

Having established a method to coreconstitute Hrd1 and Syb while maintaining fusion efficiency, I then characterized these liposomes biochemically. The four important 
factors to consider were the incorporation efficiency, coreconstitution efficiency, orientation and activity of the proteins. Incorporation efficiency was assayed by a flotation assay. Hrd1/Syb liposomes were floated in a Nycodenz step gradient and fractions were analyzed by SDS-PAGE (Figure 3.10A). Hrd1 floated primarily in fraction 2 and no Hrd1 was observed in the bottom fractions, indicating complete incorporation into liposomes. Likewise, Syb cofloated primarily in fraction 2 and no Syb was observed in the bottom fractions. Thus, both proteins were efficiently inserted into liposomes. In order to determine the orientation of Hrd1 in the liposomes, liposomes were incubated with TEV protease, which cleaves off the SBP tag and fluorescent dye on the C-terminus. In the correct orientation, $\mathrm{Hrd} 1$ is oriented with its $\mathrm{N}$ and C-termini facing the cytosol (outside) (Gardner et al., 2000). About 90\% of the Hrd1 is accessible to TEV protease, indicating that Hrd1 is primarily oriented with its $\mathrm{N}$ and C-termini facing the outside (Figure 3.10B).

A

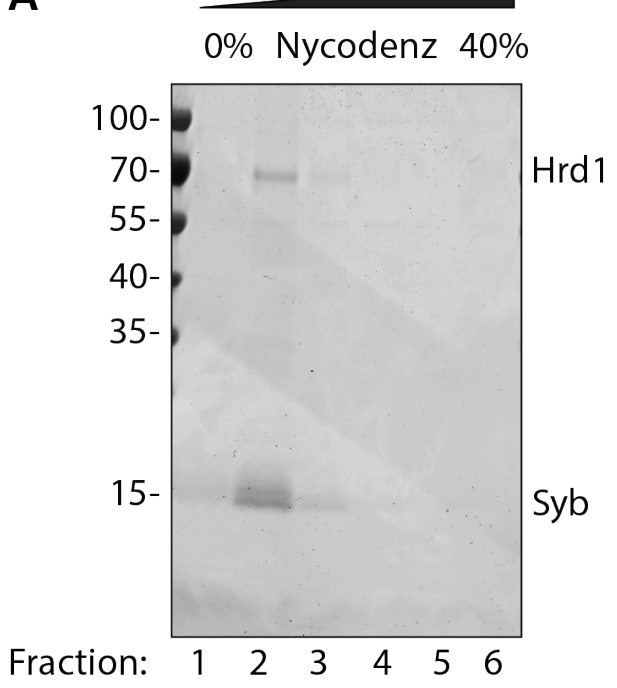

B

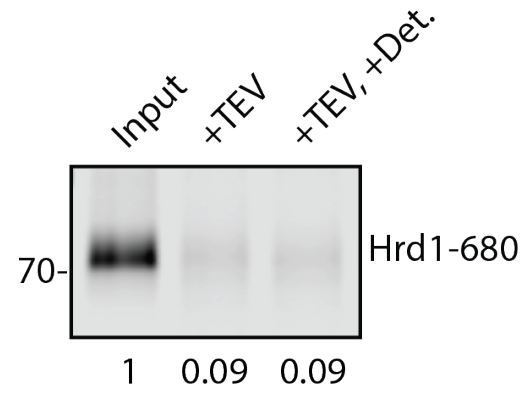

Figure 3.10: Flotation and orientation of Hrd1/Syb liposomes

A: Hrd1/Syb liposomes were subjected to flotation in a Nycodenz step gradient. Fractions were analyzed by SDS-PAGE and coomassie staining. B: Hrd1/Syb liposomes were treated with TEV protease in the presence or absence of DMNG (Det.). Samples were analyzed by SDS-PAGE and fluorescence scanning. Numbers under the lanes indicate the fraction of Hrd1 remaining normalized to the input sample.

I next checked the coreconstitution efficiency of Hrd/Syb. When coreconstituting two membrane proteins, it is important to determine the fraction of liposomes that contain both proteins. The majority of Hrd1 liposomes should contain Syb, because liposomes containing only Hrd1 would be unable to fuse and participate in retrotranslocation. To check the coreconstitution efficiency of Syb in Hrd1 liposomes, I performed a pull-down 
experiment. Hrd1/Syb liposomes were immobilized onto magnetic streptavidin beads through the SBP tag of Hrd1, and input, unbound and biotin elutions were analyzed (Figure 3.11). The biotin elution was inefficient and was therefore not included in the quantification. Quantification of the input and unbound fractions showed that $92 \%$ of Hrd1 and $75 \%$ of Syb bound to the beads (Figure 3.11, -Biotin). This indicated that the majority of Syb was localized in Hrd1-containing liposomes. As a control, beads were treated with biotin before the pull-down experiment (Figure 3.11, +Biotin). In that case, only $12 \%$ of $\operatorname{Hrd} 1$ and $10 \%$ of Syb bound to the beads, indicating that non-specific binding was low.

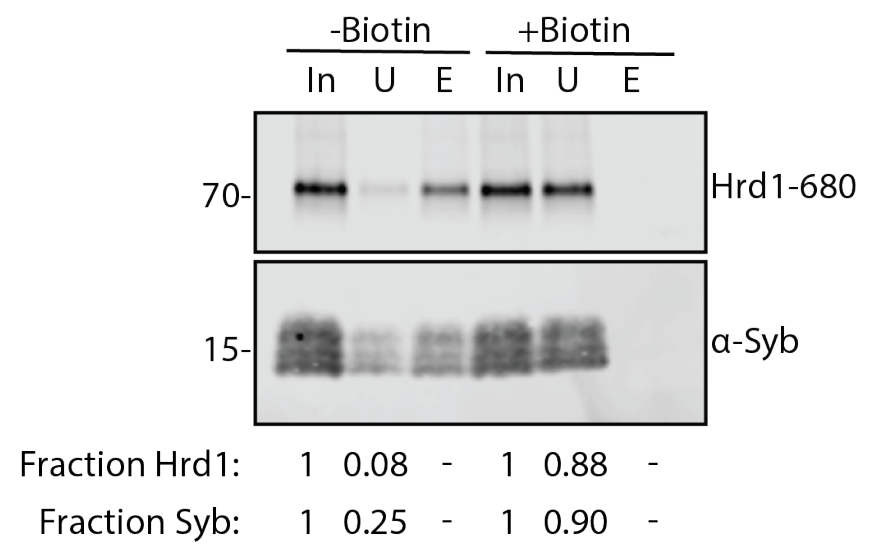

Figure 3.11: Coreconstitution efficiency of Hrd1/Syb in liposomes

Hrd1/Syb liposomes were immobilized onto magnetic streptavidin beads. Input (In), unbound (U) and biotin elutions (E) were analyzed by SDS-PAGE and fluorescence scanning (Hrd1) and immunoblotting ( $\alpha$-Syb, Synaptic Systems 69.1). As a control, beads were treated with biotin before use (+Biotin). Fraction of Hrd1 and Syb remaining were quantified and normalized to the input sample.

Hrd1 autoubiquitination is essential for its ability to degrade ERAD substrates in vivo and has been proposed to trigger a conformational change that opens the channel (Bordallo and Wolf, 1999; Baldridge and Rapoport, 2016). Therefore, Hrd1 must retain the ability to autoubiquitinate when coreconstituted with Syb. I checked Hrd1 autoubiquitination by adding components of the standard Hrd1 ubiquitination machinery (Uba1, Ubc7, Cue1-c, and ubiquitin) to Hrd1/Syb liposomes. Approximately $60 \%$ of Hrd1 autoubiquitinated after $1 \mathrm{~h}$ (Figure 3.12A, B), which is in the range of what was observed in previous reconstitutions, albeit slightly less efficient (Stein et al., 2014; Baldridge and Rapoport, 2016). The presence of Syb did not affect autoubiquitination as Hrd1-only liposomes prepared with DM showed identical autoubiquitination (Figure 3.12B). Hrd1 autoubiquitination is more efficient when reconstituted into liposomes with DMNG than with DM, indicating that DMNG is better in maintaining 
Hrd1 activity (compare Figure 3.12 to Figure 3.42). Nevertheless, DM was the best option in terms of preserving fusion efficiency while maintaining Hrd1 autoubiquitination activity (Figures 3.8, 3.9, 3.12).
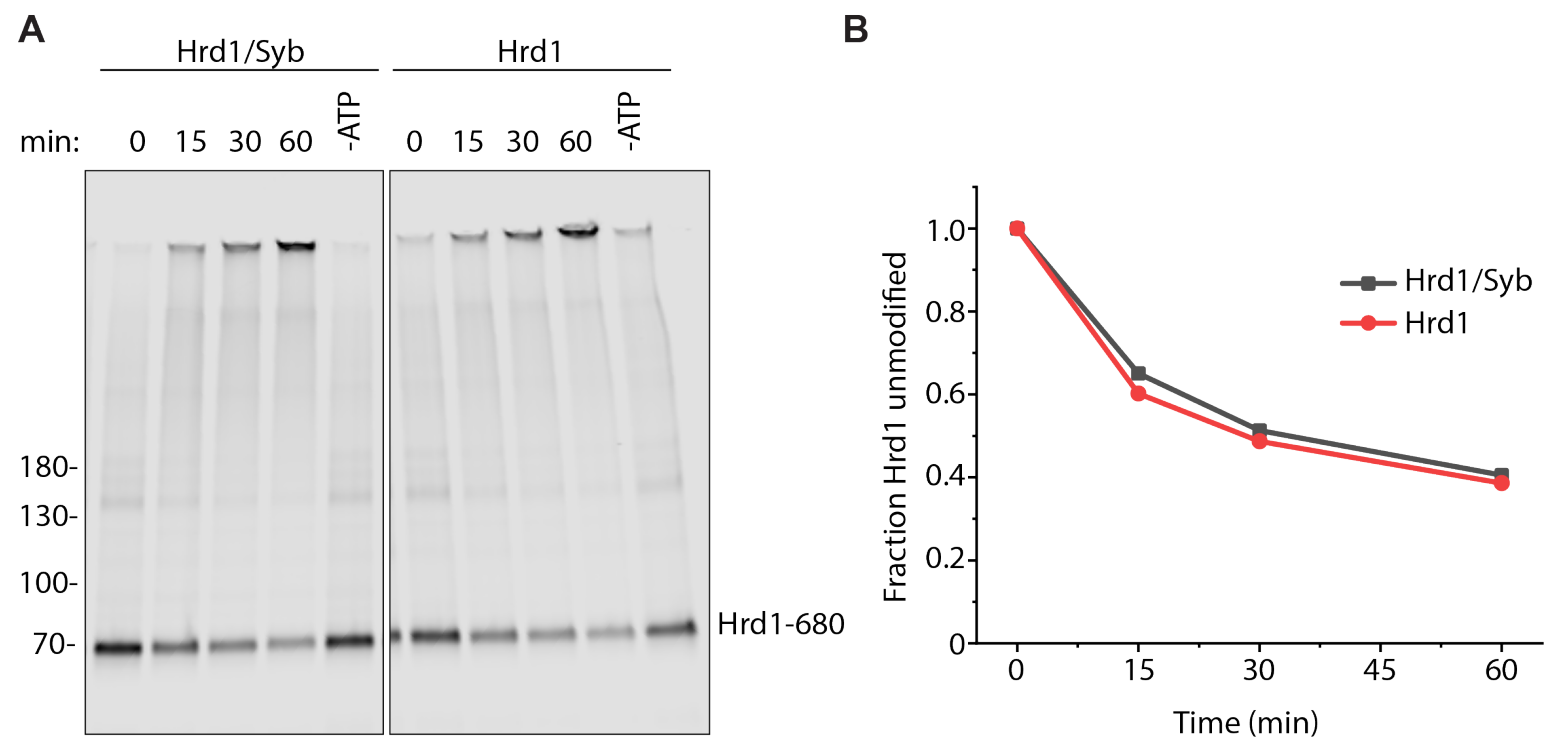

Figure 3.12: Hrd1 coreconstitution with Syb does not affect autoubiquitination activity

A : Hrd1/Syb or Hrd1 were reconstituted into liposomes with DM and were incubated with ubiquitination machinery with or without ATP for $1 \mathrm{~h}$ at $30^{\circ} \mathrm{C}$. Timepoints were taken into sample buffer and were analyzed by SDS-PAGE and fluorescence scanning. The -ATP sample was collected after 60 min. B: Quantification of Hrd1 autoubiquitination normalized to the 0 min timepoint.

\subsection{Characterizing fusion between substrate and Hrd1 liposomes}

Having established that Hrd1/Syb liposomes fuse efficiently to $\Delta$ N49 liposomes, I proceeded to encapsulate $\mathrm{CPY}^{*}$ into the $\triangle \mathrm{N} 49$ liposomes and checked how fusion efficiency was affected (as in Figure 3.7). SNARE-mediated fusion proceeds via a series of wellcharacterized intermediates. This includes tight docking of the liposomes where the bilayers are in close proximity but do not mix, a hemifused state where only the outer leaflets mix, and finally the completely-fused state where the fusion pore expands and luminal contents mix (Hernandez et al., 2012). A prerequisite of the fusion system is that fusion must go to completion, with membrane and luminal contents completely mixing. Because liposomes can be trapped in an extended hemifused state during fusion (Hernandez et al., 2012), I aimed to characterize the extent of fusion between 
substrate and Hrd1 liposomes with assays that monitored both membrane mixing as well as luminal content mixing.

\subsubsection{Fusion of Hrd1 and substrate liposomes results in efficient membrane mixing}

I first assayed fusion between Hrd1/Syb and $\mathrm{CPY}^{*} / \Delta \mathrm{N} 49$ liposomes by a lipid mixing assay. As shown in Figure 3.13, fusion was as efficient with unfloated $\mathrm{CPY}^{*} / \Delta \mathrm{N} 49$ liposomes as with $\Delta$ N49 liposomes (compare Figure 3.13A, black trace to Figure 3.9, cyan trace). Thus, the use of NiNTA lipids in the liposomes and encapsulation of

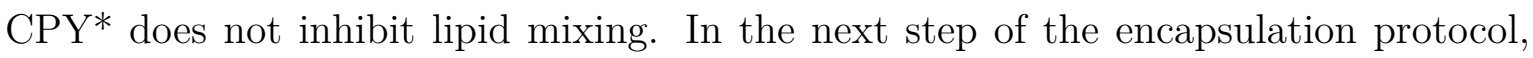
the outside-bound $\mathrm{CPY}^{*}$ is removed by flotation in a Nycodenz gradient with imidazole and urea (Figure 3.5). These floated liposomes exhibited less efficient lipid mixing than the unfloated liposomes (Figure 3.13A, blue trace). This was not due to dilution of $\mathrm{CPY}^{*} / \Delta \mathrm{N} 49$ liposomes after flotation, as the dilution factor was accounted for in the lipid mixing assay. Flotation in imidazole and urea might affect the activity of the $\Delta \mathrm{N} 49$ complex or the integrity of the liposomes. Both fusion reactions could be inhibited by incubation with $\mathrm{Syb}_{\text {sol }}$, although there was some spontaneous lipid mixing in both unfloated and floated samples (Figure 3.13A, red and green traces).

The lipid mixing assay is a bulk biophysical assay which is useful for general characterization of fusion, but it does not provide insight into the fusion of specific liposome populations. It is possible that populations of liposomes that contain only $\Delta \mathrm{N} 49$ or Syb fuse more efficiently than liposomes containing both SNAREs and Hrd1 or CPY*. I employed an alternative biochemical assay to check for delivery of $\mathrm{CPY}^{*}$ to Hrd1 liposomes. After fusion, Hrd1-containing liposomes were immobilized on streptavidin magnetic beads (Figure 3.13B). Most of the CPY* was pulled down along with Hrd1 after fusion. When fusion was inhibited, less CPY* was pulled down, indicating that $\mathrm{CPY}^{*}$ was transferred to Hrd1 liposomes in a SNARE-specific manner. However, a significant fraction of $\mathrm{CPY}^{*}$ was pulled down in the inhibited reaction and could be eluted with biotin, meaning that a fraction of $\mathrm{CPY}^{*}$-containing liposomes sticks nonspecifically to Hrd1 liposomes. A disadvantage of the pull-down assay is that it does not discriminate between docked, fused or hemifused liposomes. Any of these three possibilities would result CPY* pulldown along with Hrd1. Therefore, I continued to characterize fusion with additional assays. 
A

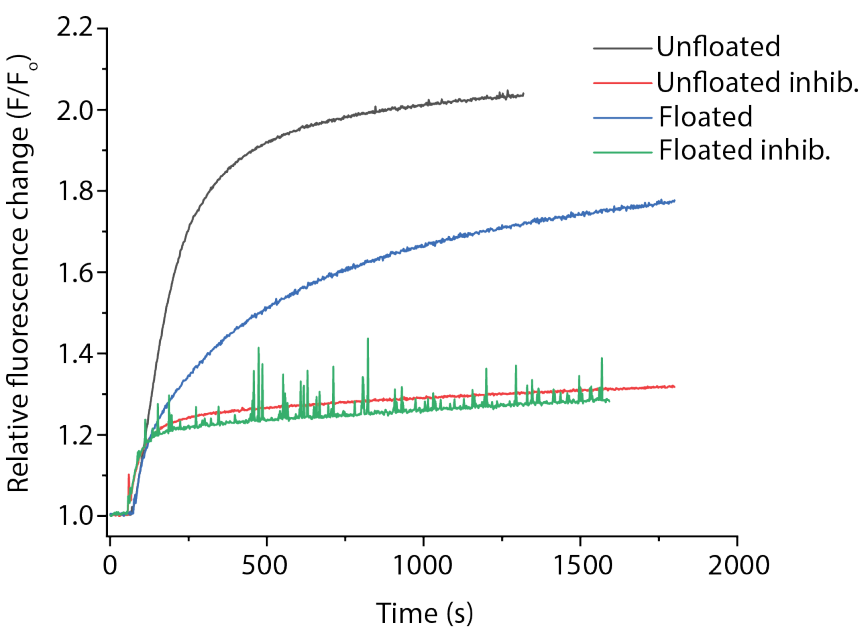

B

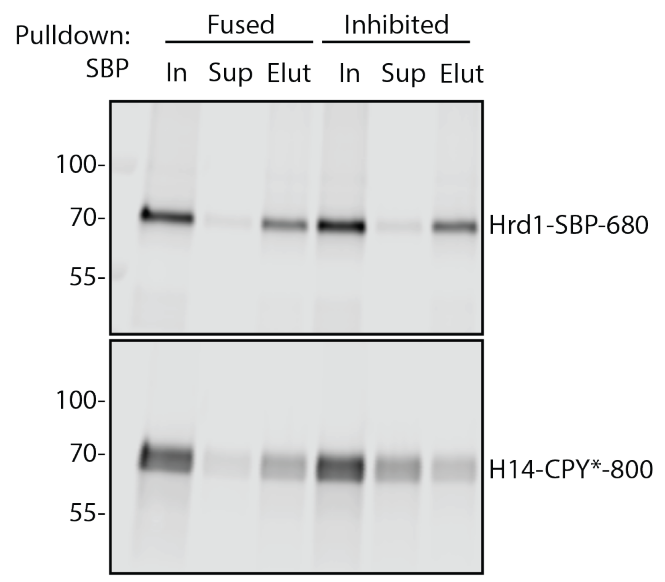

Figure 3.13: Transfer of CPY* to Hrd1 liposomes after fusion

A: Lipid mixing assay of Hrd1/Syb and $\mathrm{CPY}^{*} / \Delta \mathrm{N} 49$ liposomes before and after flotation in a Nycodenz step gradient. Inhib: fusion was inhibited by incubation of $\mathrm{CPY}^{*} / \Delta \mathrm{N} 49$ liposomes with $\mathrm{Syb}_{\text {sol }}$ prior to fusion. B: Hrd1/Syb liposomes were immobilized onto magnetic streptavidin resin after fusion with $\mathrm{CPY}^{*} / \Delta \mathrm{N} 49$ liposomes, or after fusion was inhibited with $\mathrm{Syb}_{\text {sol }}$. Input (In), unbound (Sup) and biotin elutions (Elut) were analyzed by SDS-PAGE and fluorescence scanning.

Having observed efficient lipid mixing, I then asked how well the fusion system could transfer membrane proteins between populations of liposomes. I developed a biochemical assay involving Hrd1 autoubiquitination as a readout (Figure 3.14A). Briefly, Hrd1/Syb liposomes were fused with $\Delta$ N49 liposomes coreconstituted with the E2 ubiquitin conjugating enzyme Ubc6. Ubc6 is a tail-anchored E2 enzyme localized to the ER membrane and functions in conjunction with the E3 ligase Doa10 (Sommer and Jentsch, 1993; Swanson et al., 2001). It has been shown to prime Doa10 substrates by monoubiquitination (Weber et al., 2016). Genetic experiments have also implicated Ubc6 in Hrd1-mediated degradation of CPY* (Hiller et al., 1996).

In order to catalyze autoubiquitination, the RING domain of Hrd1 requires an E2 enzyme loaded with ubiquitin in close proximity. Ubc6 is the only E2 enzyme in this reaction, and is localized in the membrane of a separate population of liposomes. Therefore, Hrd1 autoubiquitination can only occur if Ubc6 is delivered to Hrd1 liposomes by fusion. Liposomes were fused and autoubiquitination was initiated by adding E1 enzyme and ATP. Hrd1 autoubiquitinated efficiently upon fusion with Ubc6 liposomes, reaching $80 \%$ ubiquitination efficiency after $60 \mathrm{~min}$, with about $50 \%$ ubiquitinated after 10 min (Figure 3.14B, C). By comparison, when fusion was inhibited with $\mathrm{Syb}_{\mathrm{sol}}$, Hrd1 autoubiquitination was noticeably less efficient. Hrd1 also ubiquitinated Ubc6, 
and this was more efficient in the fused sample compared to the inhibited fusion sample (Figure 3.14B, C). No ubiquitination of Hrd1 or Ubc6 was observed in the absence of ATP. These results indicated that the fusion system successfully delivers membrane components to Hrd1, and that Hrd1 retains ubiquitination activity after fusion. There was a fraction of Hrd1 and Ubc6 that was ubiquitinated in the inhibited fusion control. This could be explained by Ubc6 having some activity in trans, where it might catalyze ubiquitination from an opposing membrane.

This result also established that Ubc6 can act as an E2 enzyme for Hrd1 in a reconstituted system, which has been implied in previous studies but never directly shown (Hiller et al., 1996). Of note, Ubc6 primarily catalyzed monoubiquitination of Hrd1, which is evident by the main size shift corresponding to the size of a single ubiquitin. This is in agreement with previous observations that the function of Ubc6 is to prime substrates by monoubiquitination, which is followed by chain elongation by Ubc7 (Weber et al., 2016). 
A
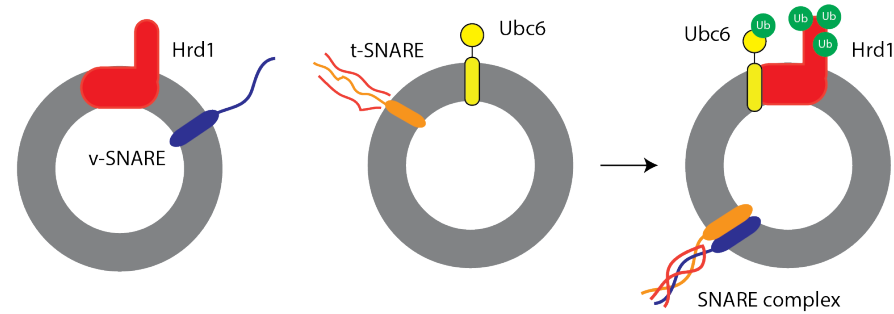

B
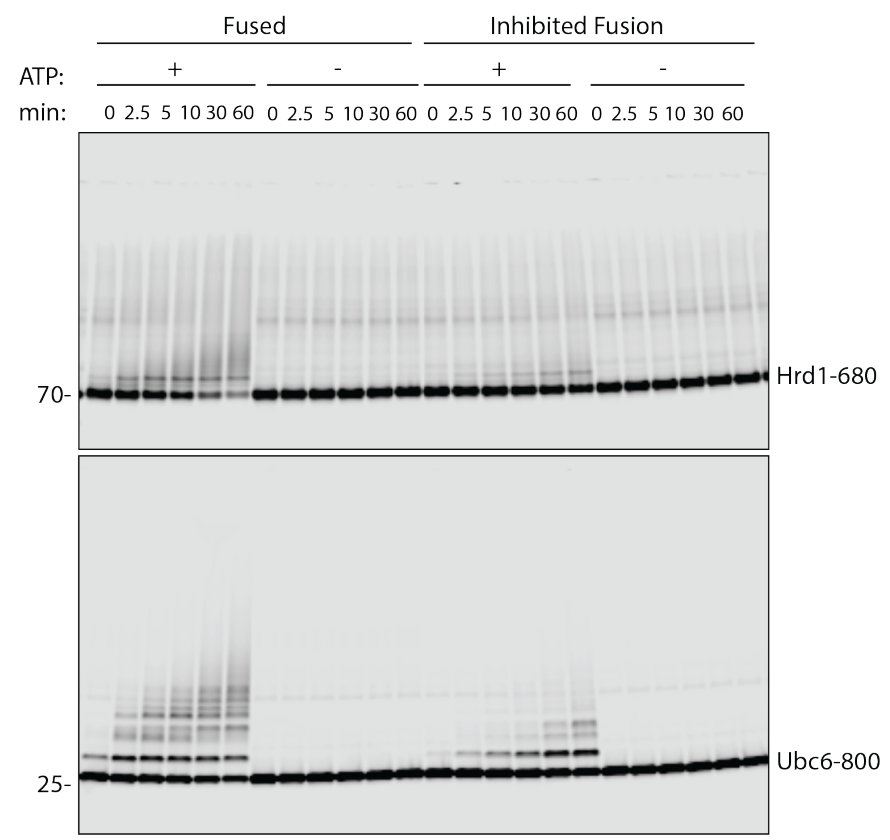

C

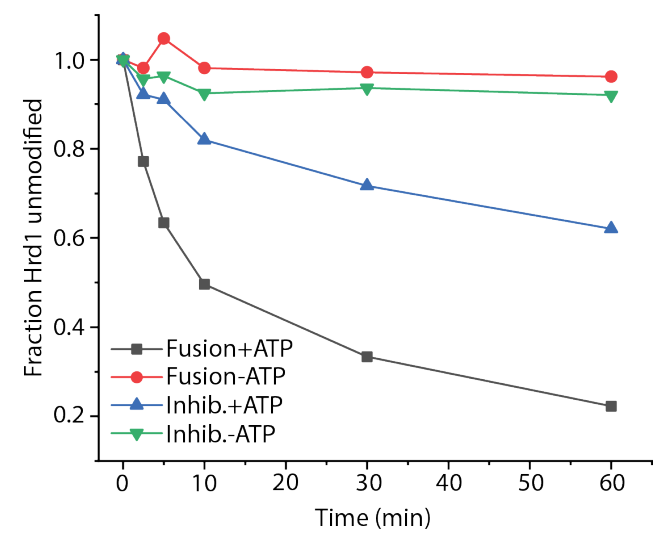

Figure 3.14: Hrd1 autoubiquitinates upon fusion with Ubc6 liposomes A: Scheme of fusion assay using the E2 enyzme Ubc6 and Hrd1. Hrd1/Syb liposomes were fused with Ubc6/ $\Delta$ N49 liposomes and ubiquitination was initiated by adding E1 enzyme and ATP. B: Ubiquitination reaction after fusion. Samples were taken at indicated timepoints and analyzed by SDS-PAGE and fluorescence scanning. Where indicated, fusion was inhibited with $\mathrm{Syb}_{\text {sol }}$ or ATP was omitted. Note that Hrd1 autoubiquitination is notably more efficient after fusion. C: Quantification of Hrd1 autoubiquitination normalized to the 0 min timepoint.

\subsubsection{Fusion of Hrd1 and substrate liposomes leads to luminal content mixing}

After establishing that membrane mixing occurs efficiently in the fusion system, I characterized the luminal content mixing. Failure to mix luminal contents sufficiently would be a bottleneck in the subsequent retrotranslocation experiments. There are many existing methods to monitor luminal content mixing. The standard methods rely on encapsulation of fluorescent dyes at very high concentrations, which exhibit dequenching upon fusion with liposomes containing no fluorescent dye (van den Bogaart 
et al., 2010). Other assays have used FRET between encapsulated dyes or even between larger molecules like DNA oligonucleotides or GFP (Liu et al., 2017; Diao et al., 2010; Heo et al., 2017). Assays using fluorescent dyes are prone to leakage, whereas assays using encapsulated oligonucleotides do not establish that the fusion pore is large enough to accommodate larger proteins. Content mixing assays using GFP are better because the fusion pore must be large enough for a protein of at least $25 \mathrm{kDa}$ to pass through (Heo et al., 2017).However, the GFP assay developed by Heo et al, as well as all other assays mentioned, rely on FRET or fluorescence dequenching as the readout. Like the lipid mixing assay, these content mixing assays are suitable for assessing bulk fusion, but they give no information about different subpopulations of liposomes or the activity of the proteins encapsulated within them.

Therefore, I developed a biochemical content mixing assay that reports on content mixing of liposomes containing an encapsulated substrate, as well as the activity and solubility of the encapsulated proteins. As shown in Figure 3.15, the ERAD-L substrate short $\mathrm{CPY}^{*}\left(\mathrm{sCPY}^{*}\right)$, with an N-terminal His ${ }_{14}$-SUMO tag and a C-terminal GFP tag labeled with DL800, was encapsulated into liposomes using the NiNTA technique (as described in section 3.2.2). The substrate $\mathrm{sCPY}^{*}$ is a truncated version of $\mathrm{CPY}^{*}$, where the first 52 amino acids of CPY* are fused to its last 75 amino acids (Carvalho et al., 2010; Stein et al., 2014). Importantly, sCPY* is a bona fide ERAD-L substrate and behaves similarly to $\mathrm{CPY}^{*}$, both in vivo in degradation experiments and in vitro in reconstitutions with Hrd1 (Carvalho et al., 2010; Stein et al., 2014). SUMO (small ubiquitin-like modifier) is a ubiquitin-like protein which is added to lysine residues of proteins post-translationally, and plays various roles in cell cycle progression and nucleocytoplasmic transport (Wilkinson and Henley, 2010). The SUMO tag can be efficiently cleaved by the SUMO protease Ulp1, which recognizes the SUMO fold and cleaves after its C-terminal diglycine motif (Li and Hochstrasser, 1999). The SUMO and GFP tags on sCPY* improved the solubility of the aggregation-prone sCPY* (Stein et al., 2014).

His-tagged Ulp1 was encapsulated using the NiNTA method and was fused to liposomes containing encapsulated His-SUMO-sCPY*-GFP. If fusion leads to content mixing, the substrate will become accessible to Ulp1 cleavage (Figure 3.15A), which is monitored by a size shift in SDS-PAGE. As evident in Figure 3.15B, SUMO-sCPY* was cleaved during the course of fusion. The cleavage kinetics were similar to previously-observed lipid mixing kinetics (Figure 3.13, blue trace). This indicates that Ulp1 cleavage, which is normally extremely fast (Frey and Gorlich, 2014), was rate-limited by fusion. When fusion was inhibited with soluble Syb, no cleavage was observed over 60 min (Figure 
$3.15 \mathrm{~B})$. Cleavage was slightly faster at 30 and $37^{\circ} \mathrm{C}$ compared to $25^{\circ} \mathrm{C}$, in agreement with previous observations that lipid mixing is temperature-dependent (Hernandez et al., 2012). Approximately 65-70\% of sCPY* was cleaved after 60 min (Figure 3.15C), indicating that about $30-35 \%$ of substrate-containing liposomes either did not fuse, or fused with liposomes that did not contain Ulp1.

To conclude, the SUMO-cleavage content mixing assay demonstrated that fusion went to completion in this system, with luminal contents efficiently mixed between two populations of liposomes.

A
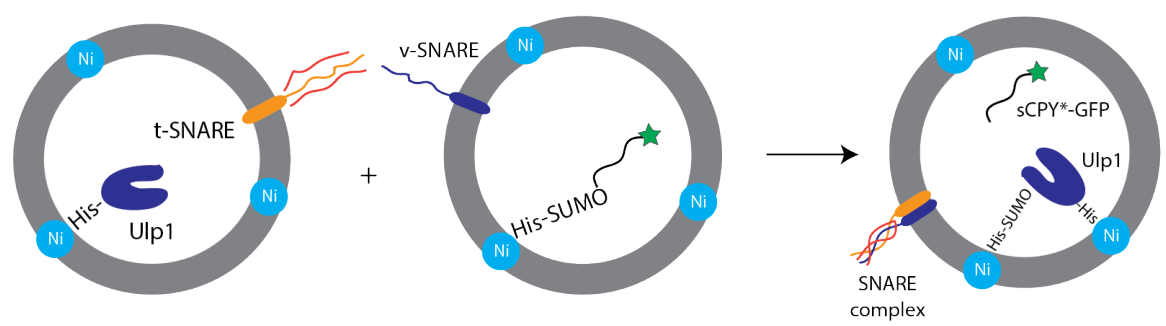

B

C
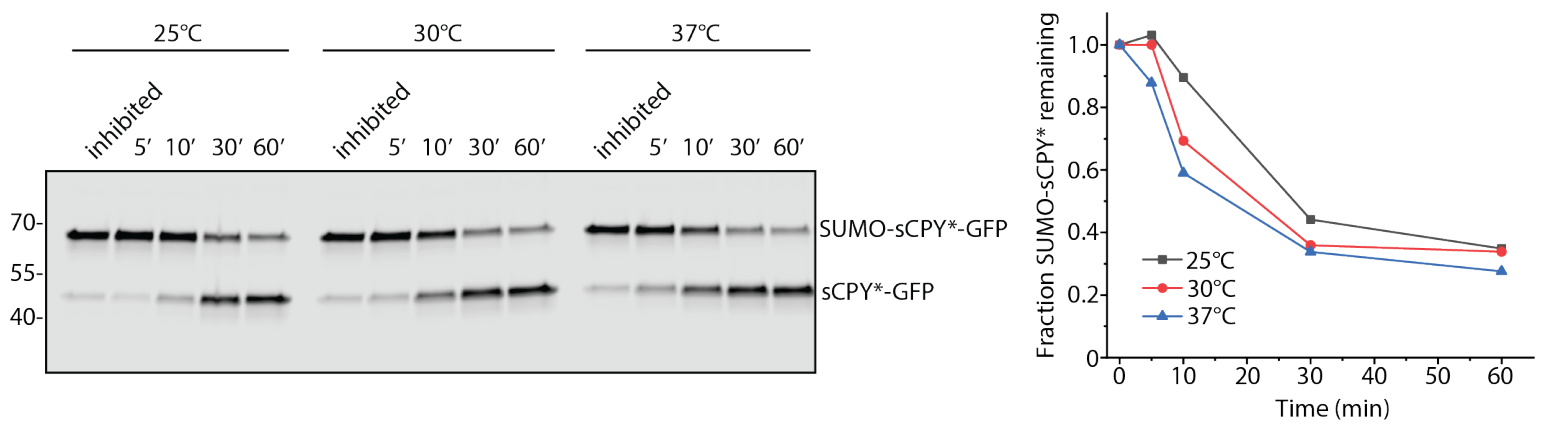

Figure 3.15: Fusion system mixes luminal contents efficiently

A: Scheme of the luminal content mixing assay. His-tagged Ulp1 was encapsulated into liposomes with $\Delta$ N49 using the NiNTA method. These liposomes were fused with liposomes containing encapsulated His-SUMO-sCPY*-GFP along with Syb, also prepared with the NiNTA method. Upon fusion and content mixing, the substrate bcomes accessible to Ulp1, which cleaves off the SUMO tag. B: Timepoints were taken during fusion into SDS sample buffer to quench the reaction. Fusion was performed at different temperatures. Where indicated, fusion was inhibited by incubating Ulp1 liposomes with $\mathrm{Syb}_{\mathrm{sol}}$. The inhibited reaction was analyzed after $60 \mathrm{~min}$. Samples were analyzed by SDS-PAGE and fluorescence scanning. C: Quantification of HisSUMO-sCPY*-GFP remaining (top band), normalized to the inhibited fusion after 60 min. 


\subsection{Retrotranslocation experiments using the fusion system}

\subsubsection{CPY* was not retrotranslocated by Hrd1 after fusion}

Having established that the fusion system efficiently delivered luminal contents to Hrd1 liposomes, I asked whether Hrd1 could retrotranslocate encapsulated CPY*. To this end, I fused CPY*/ $\mathrm{CN}^{*} 4$ liposomes with Hrd1/Syb liposomes, and added ubiquitination machinery along with ATP. Because luminal CPY* can only be ubiquitinated if it becomes exposed to the outside, the ubiquitination status of the CPY* was monitored as a readout for retrotranslocation. About $85 \%$ of $\mathrm{CPY}^{*}$ was encapsulated (Figure 3.7), meaning that retrotranslocation could be concluded only if more than $15 \%$ of $\mathrm{CPY}^{*}$ became ubiquitinated. Ubiquitination levels in the range of $0-15 \%$ could originate from outside-bound CPY*.

Consistently, only $15 \%$ of CPY* was ubiquitinated after fusion (Figure 3.16A, B), which corresponded with the fraction that was unprotected from proteinase K (Figure 3.7). Therefore, it could not be concluded that $\mathrm{CPY}^{*}$ was retrotranslocated by Hrd1. Hrd1 autoubiquitinated to approximately 50\% efficiency after fusion, which was slightly less efficient than observed in unfused liposomes (Figure 3.12). There are many possible reasons why $\mathrm{CPY}^{*}$ was not retrotranslocated by $\mathrm{Hrd} 1$ in this experiment. The next sections deal with these problems individually. 
A

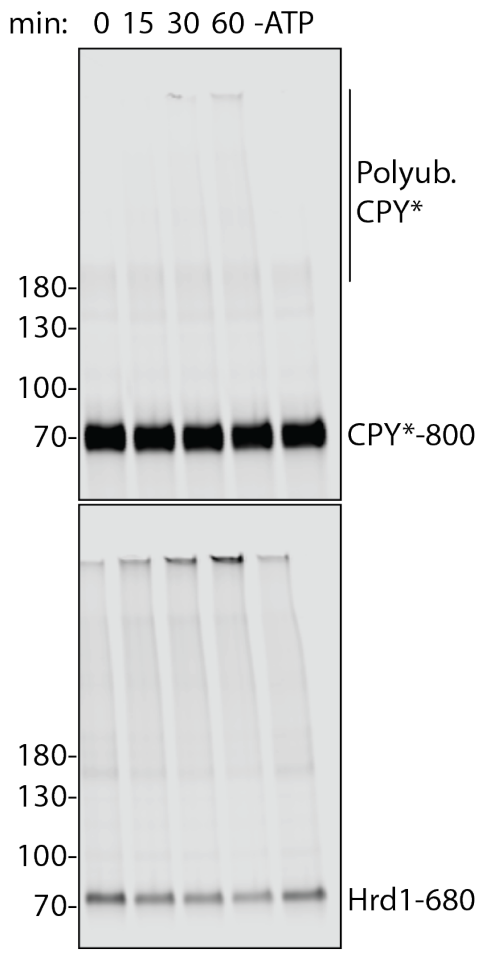

B

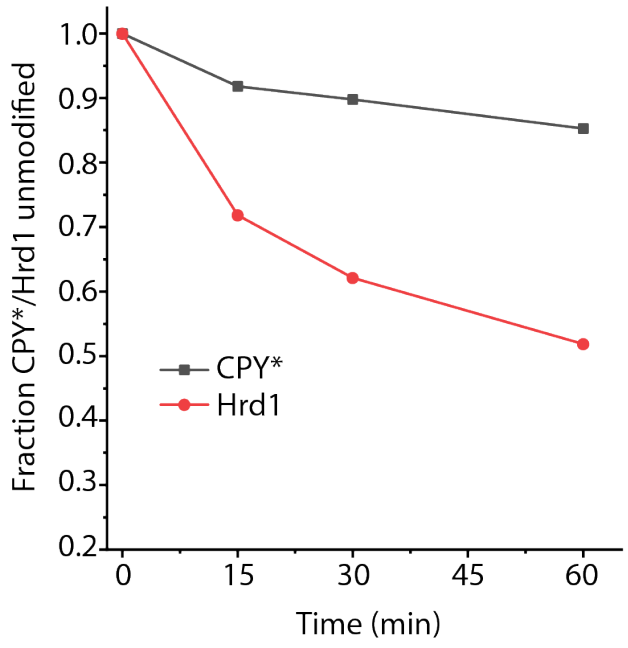

Figure 3.16: Encapsulated H14-CPY* does not get retrotranslocated by Hrd1 after fusion

A: His $_{14}{ }^{-} \mathrm{CPY}^{*} / \Delta \mathrm{N} 49$ and Hrd/Syb liposomes were fused and mixed with ubiquitination machinery. ATP was added to start the reaction. Time points taken during the ubiquitination reaction were analyzed by SDS-PAGE and fluorescence scanning. The -ATP sample was taken after 60 min. B: Quantification of $\mathrm{CPY}^{*}$ and Hrd1 ubiquitination, normalized to 0 min timepoint.

\subsubsection{C-terminal tethered CPY* was possibly retrotranslocated}

In the NiNTA encapsulation method, the encapsulated substrate remains bound or tethered to NiNTA lipids in the inner bilayer. Although this was a necessary step in order to efficiently encapsulate substrates, the tethering could potentially cause issues in retrotranslocation. It may be important in which orientation substrates are presented to the luminal face of Hrd1, as there may be a preferred site for insertion into the channel. It was previously demonstrated that attachment of a tightly-folded DHFR domain to the C-terminus of CPY* delayed its degradation, whereas attachment to the N-terminus did not (Bhamidipati et al., 2005), raising the possibility that there is a preferred directionality of $\mathrm{CPY}^{*}$ insertion into the retrotranslocon. However, this study did not show whether CPY*-DHFR was actually imported into the ER. In the previous retrotranslocation experiments, I used an N-terminal His-tagged CPY* (Figure 3.16). If the N-terminal region of $\mathrm{CPY}^{*}$ is preferred for insertion into the channel, this may 
be inhibited by N-terminal tethering. To test for this possibility, I swapped the His 14 tag to the C-terminus of $\mathrm{CPY}^{*}\left(\mathrm{CPY}^{*}{ }_{-} \mathrm{H}_{14}\right)$. CPY*-H14 behaved identically to Nterminally-tagged $\mathrm{His}_{14}$-CPY* $^{*}$ during reconstitution with $\Delta \mathrm{N} 49$ (Figure $3.17 \mathrm{~A}$ ) and was efficiently encapsulated into liposomes, with $82 \%$ protected from proteinase $\mathrm{K}$ (Figure 3.17B).
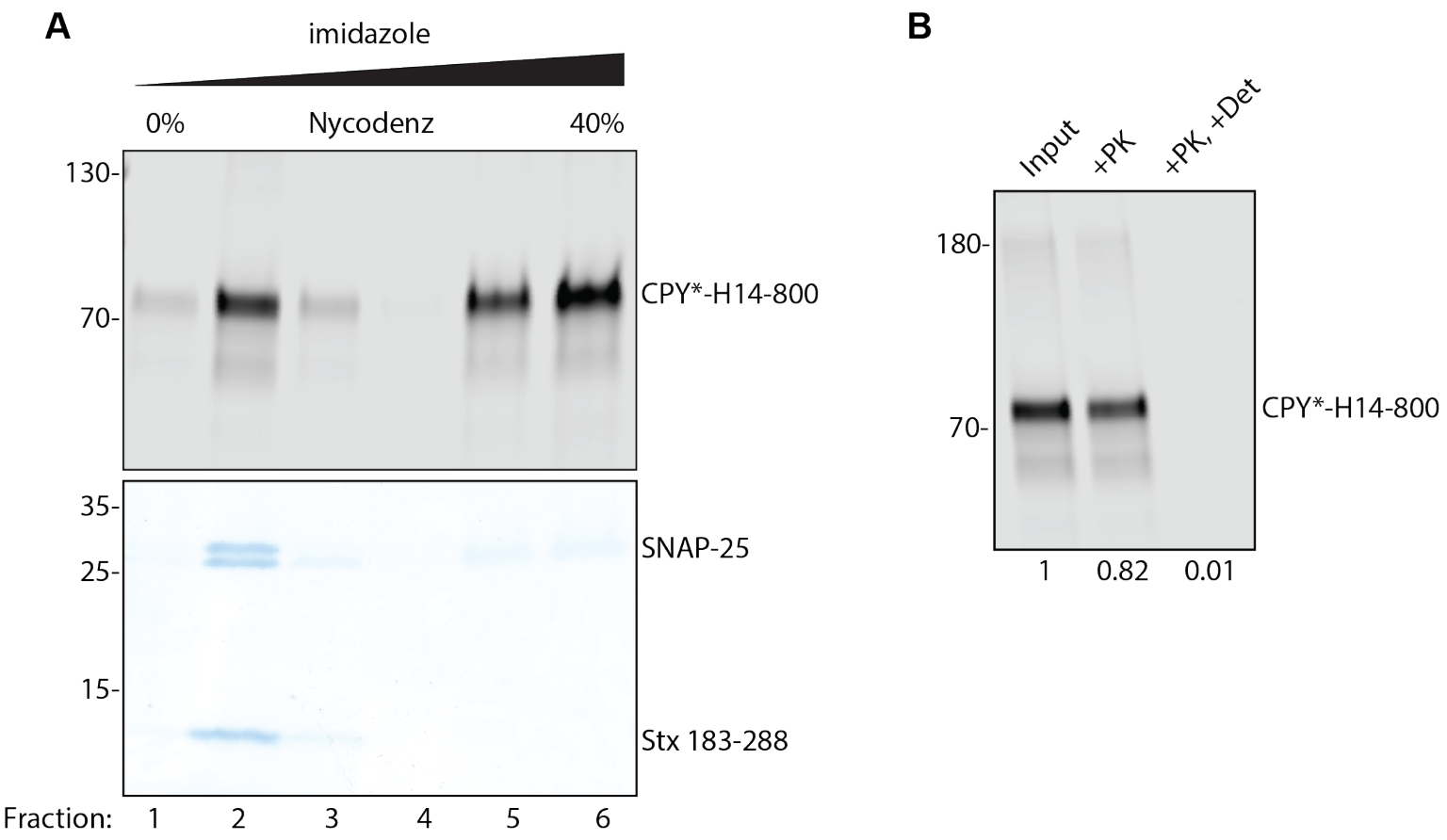

Figure 3.17: C-terminally tagged $\mathrm{CPY}^{*}-\mathrm{H}_{14}$ is efficiently encapsulated into liposomes

CPY* with a C-terminal His $_{14}$ tag $\left(\mathrm{CPY}^{*}\right.$-H14) was encapsulated along with $\Delta \mathrm{N} 49$ using the NiNTA method. A: Flotation of CPY*-H14/ ${ }^{*}$ N49 liposomes in a Nycodenz gradient with $400 \mathrm{mM}$ imidazole. Outside-bound CPY*-H14 remained in the bottom fractions, while encapsulated CPY*-H14 cofloated with the lipids. SNAP-25 and Stx 183-288 are components of the $\Delta \mathrm{N} 49$ complex. B: Proteinase K protection of floated liposomes (fraction 2). Det: Triton X-100 was added to solubilize the liposomes. Numbers below indicate the fraction of protected CPY*-H14.

Upon fusion and addition of ubiquitination machinery, roughly $25 \%$ of $\mathrm{CPY}^{*}$-H14 was ubiquitinated (Figure 3.18A, B). Approximately 15\% was ubiquitinated in the inhibited fusion control. When accounting for the inhibited fusion control and the protease protection, which showed that $18 \%$ of $\mathrm{CPY}^{*}$ was on the outside (Figure $3.17 \mathrm{~B}$ ), up to $10 \%$ of CPY*-H14 may have been retrotranslocated. More repetitions of the experiment are required to determine the significance of this result, as well as additional controls for leakage during fusion. However, I decided to focus on improving the efficiency of retrotranslocation because I aimed to use the system to characterize mechanistic details, 
and $10 \%$ retrotranslocation was an inefficient starting point. Hrd1 remained active after fusion, as $55 \%$ of Hrd1 autoubiquitinated after 1 hour, and this was independent of fusion (Figure 3.18A, C).

A

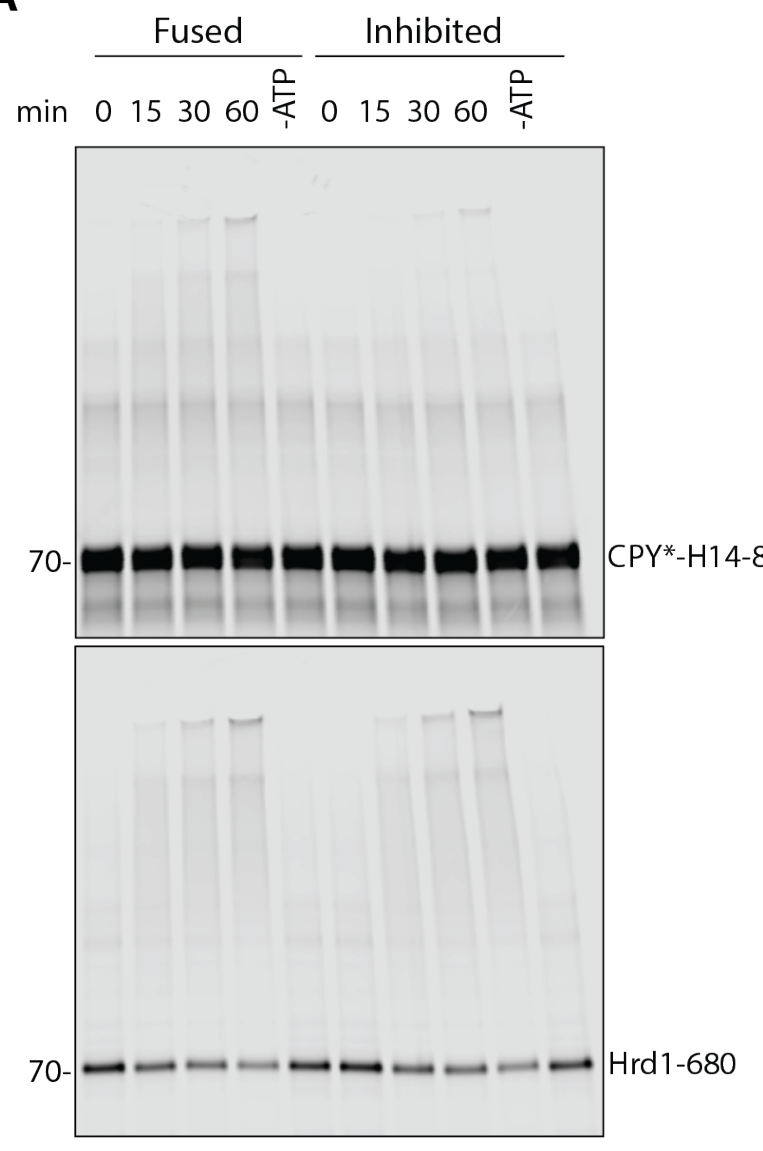

B

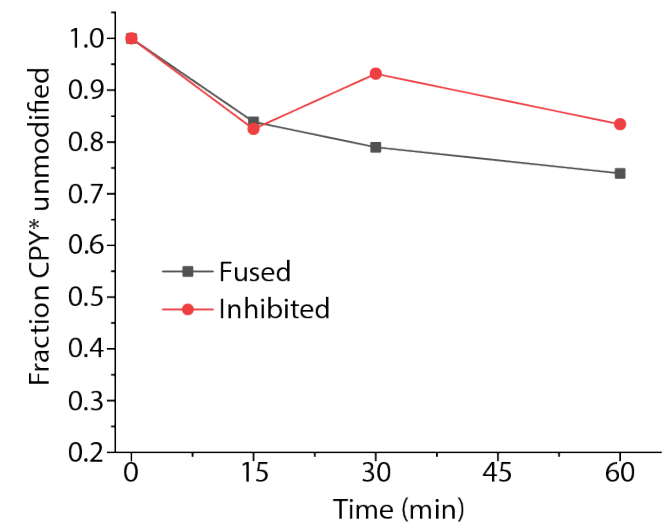

C

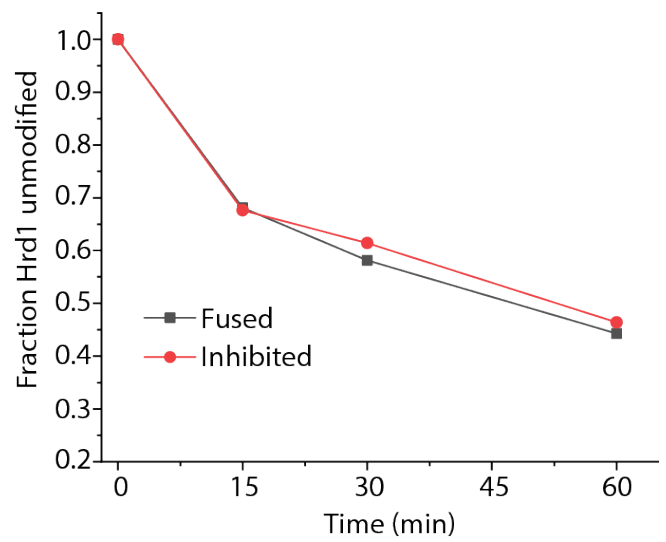

Figure 3.18: CPY*-H14 is potentially inefficiently retrotranslocated after fusion

A: $\mathrm{CPY}^{*}$-His $_{14} / \Delta \mathrm{N} 49$ and Hrd/Syb liposomes were fused and mixed with ubiquitination machinery. ATP was added to start the reaction. Where indicated, fusion was inhibited by incubating $\mathrm{CPY}^{*}-\mathrm{H}_{14} / \Delta \mathrm{N} 49$ with $\mathrm{Syb}_{\text {sol }}$. Time points taken during the ubiquitination reaction were analyzed by SDS-PAGE and fluorescence scanning. B: Quantification of ubiquitination of $\mathrm{CPY}^{*}-\mathrm{H} 14$ and $\operatorname{Hrd1}(\mathbf{C})$, normalized to the 0 min timepoint.

\subsubsection{Untethered sCPY*-GFP was not retrotranslocated by Hrd1}

Because N-terminally tethered $\mathrm{CPY}^{*}$ did not get retrotranslocated by Hrd1 and Cterminally tethered $\mathrm{CPY}^{*}$ was potentially only inefficiently retrotranslocated, I asked if an untethered substrate gets more efficiently retrotranslocated. To test an untethered luminal substrate, I used the H14-SUMO-tagged sCPY*-GFP that could be cleaved 
upon fusion by encapsulated Ulp1 (described in section 3.4.2, Figure 3.15). Substrate liposomes were fused with Hrd1 liposomes containing encapsulated Ulp1. After fusion, liposomes were floated in a Nycodenz gradient with imidazole to remove outsidebound substrate and substrate that might have leaked out of the liposomes during fusion (Figure 3.19A). Liposomes were harvested from the flotation and incubated with ubiquitination machinery (Figure 3.19B). The Ulp1 cleavage was efficient, generating sCPY*-GFP in the fused sample and not in the inhibited fusion control. However, ubiquitination efficiency of the sCPY*-GFP was inefficient and was similar to the inhibited fusion control (Figure 3.19C). Therefore, Hrd1 did not retrotranslocate untethered sCPY*GFP.

A
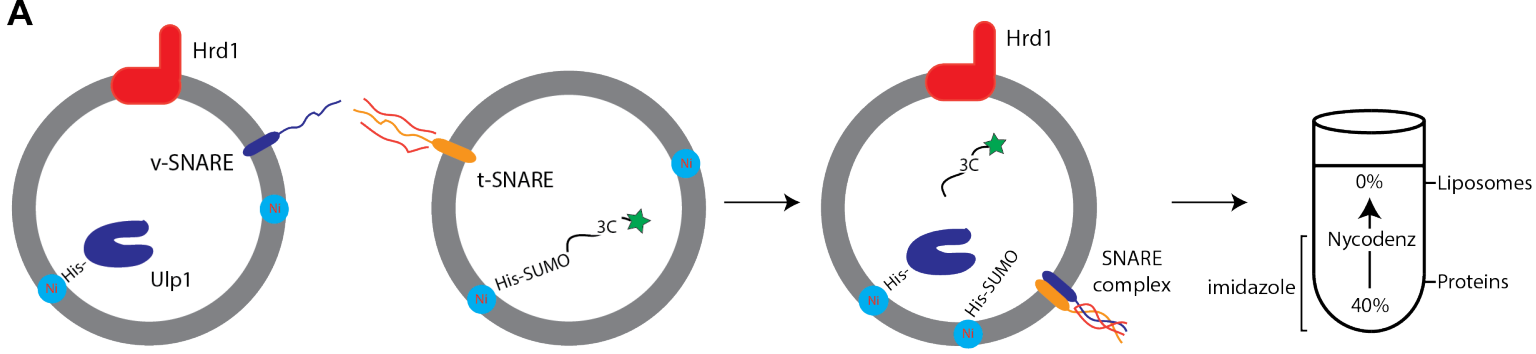

B

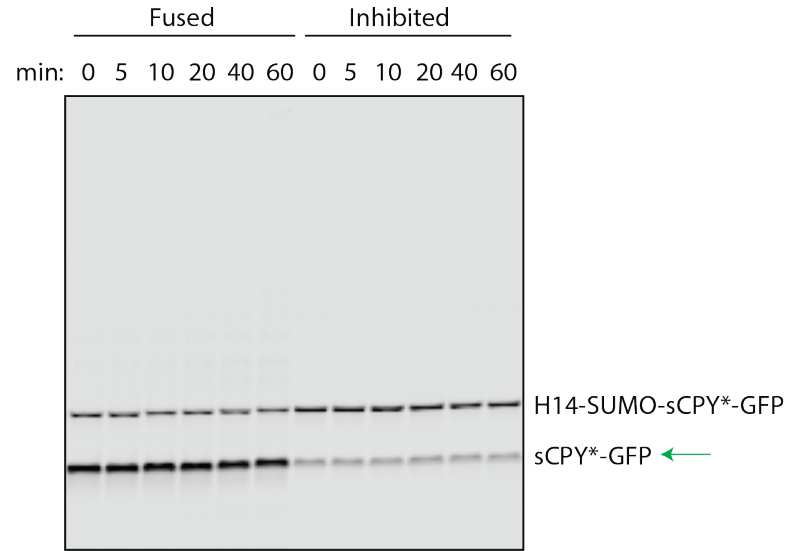

C

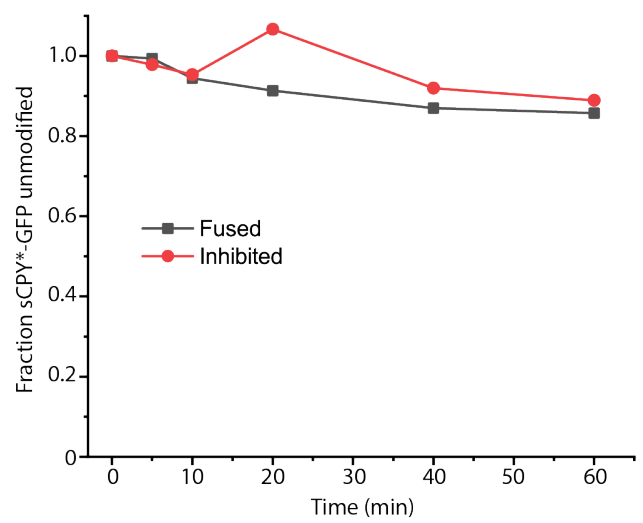

Figure 3.19: Encapsulated sCPY*-GFP is not retrotranslocated after cleavage from membrane

A: scheme of fusion and ubiquitination using the substrate His-SUMO-sCPY*-GFP and Ulp1. The substrate was encapsulated with $\Delta$ N49 using the NiNTA method. HisUlp1 was encapsulated into NiNTA-containg Hrd1 liposomes with Syb. Liposomes were fused, floated in a Nycodenz gradient containing imidazole, and then ubiquitinated. B: Ubiquitination time-course of fused liposomes. Fusion was inhibited by incubating substrate liposomes with Syb. C: Quantification of ubiquitination the bottom band (cleaved $\mathrm{sCPY}^{*}$-GFP, indicated by green arrow). 


\subsubsection{Fusion causes leakage of substrate}

It is important in the fusion system that the substrate remains encapsulated throughout fusion. To check for leakage after fusion, I cloned a $3 \mathrm{C}$ protease cleavage site before the C-terminal sortase tag in His-SUMO-sCPY*-GFP. Cleavage by $3 \mathrm{C}$ protease removes the fluorescent dye and causes a loss of fluorescence when analyzed by SDS-PAGE. His-SUMO-sCPY*-GFP/ $\triangle$ N49 liposomes were fused with Hrd1/Ulp1/Syb liposomes and floated in a Nycodenz/imidazole gradient to remove outside-bound and leaked substrate (Figure 3.20A). The liposomes were harvested and subjected to a 3C protease protection. In the fused sample, all of the SUMO-cleaved sCPY*-GFP was accessible to $3 \mathrm{C}$ protease, matching the amount remaining in the detergent control (Figure 3.20B, lower band). When fusion was inhibited, the full-length substrate was completely protected from $3 \mathrm{C}$ protease. There was some SUMO-cleaved substrate present in the inhibited fusion control, which was accessible to 3C protease. This was most likely the fraction of outside-bound substrate remaining after encapsulation, which was then cleaved by residual Ulp1 on the outside of Hrd1 liposomes.

Thus, fusion resulted in leakage of the encapsulated substrate. Although the sCPY* leaked during fusion, it cofloated with the liposomes, showing that it was somehow still associated with the liposomes. It is possible that the SUMO-cleaved substrate leaks to the outside of the liposomes during fusion and then aggregates and sticks to the liposome membrane. This would explain why it is not ubiquitinated by Hrd1 (Figure 3.19). The leakage was not caused by flotation in Nycodenz, because the substrate was protected in the inhibited fusion control liposomes, which were also floated. 


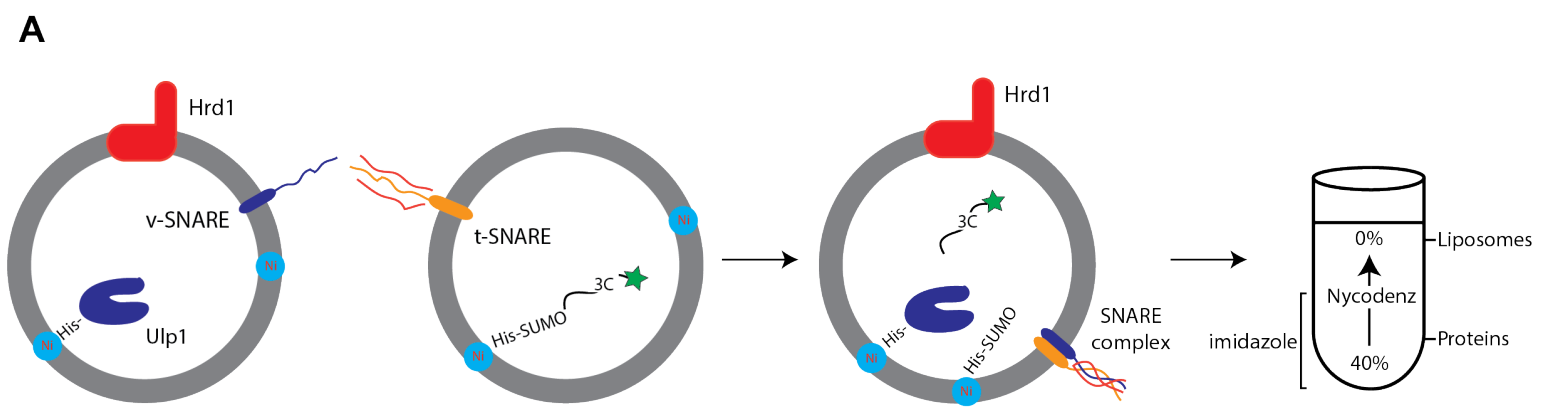

B

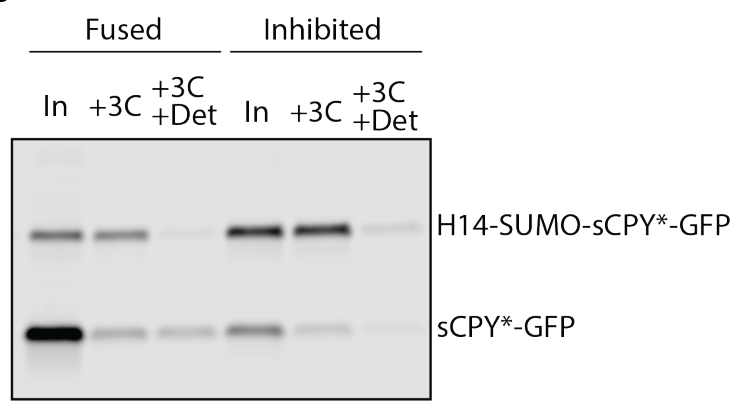

Figure 3.20: Ulp1-cleaved sCPY*_GFP leaks out of liposomes during fusion A: His-SUMO-sCPY*-GFP/ $\triangle$ N49 liposomes were fused with Hrd1/Ulp1/Syb liposomes and floated in a Nycodenz/imidazole gradient. The sCPY* construct contained a $3 \mathrm{C}$ cleavage site on its C-terminus before the sortase tag, which contains the DL800 fluorescent dye. B: Liposomes were harvested from the gradient and were treated with 3C protease either with or without DMNG (Det). In: input, where no protease was added. The reactions were analyzed by SDS-PAGE and fluorescence scanning.

\subsubsection{Leakage during fusion is dependent on protein:lipid ratio}

To gain further insight into leakage during fusion, I asked whether leakage was specific to Hrd1 or whether it was dependent on the protein concentration in the liposomes. For this, I fused His-SUMO-sCPY*-GFP/Syb liposomes with either Hrd1/ $\Delta$ N53, Doa10/ $\Delta$ N53, or $\Delta$ N53-only liposomes. $\Delta \mathrm{N} 53$ is a variant of the $\Delta$ N49 acceptor SNARE complex, which fuses efficiently with Syb liposomes in vitro (Hernandez et al., 2012). Doa10 is also a membrane-embedded ubiquitin ligase involved in ERAD, but it is not involved in $\mathrm{CPY}^{*}$ degradation (Swanson et al., 2001). It served as a control for overall protein concentration in the membrane.

Fusion was performed in the presence of excess Ulp1 added to the outside, so that any leaked substrate would immediately be cleaved (Figure 3.21B). The substrate was cleaved over time in the fused samples but not in the inhibited fusion controls (Figure 3.21A). The cleavage kinetics were similar to lipid mixing kinetics, indicating that 
leakage happened during the fusion process (Figure 3.21C). The observed effect was indeed leakage and not retrotranslocation by Hrd1, as Doa10 liposomes also resulted in similar cleavage during fusion. Interestingly, there was minimal leakage when substrate liposomes were fused to $\Delta$ N53-only liposomes, indicating that fusion using Syb and the $\Delta \mathrm{N}$ complex is not inherently leaky. Rather, the high protein:lipid ratio in the Hrd1 and Doa10 liposomes likely contributed to membrane instability during fusion. Here they were reconstituted at protein:lipid ratios of 1:1000. Considering that Hrd1 and Doa10 contain 8 and 14 transmembrane domains, respectively (Schoebel et al., 2017; Kreft et al., 2005), they contribute significantly greater protein density in the membrane than the single-TM SNAREs at a similar protein:lipid ratio.

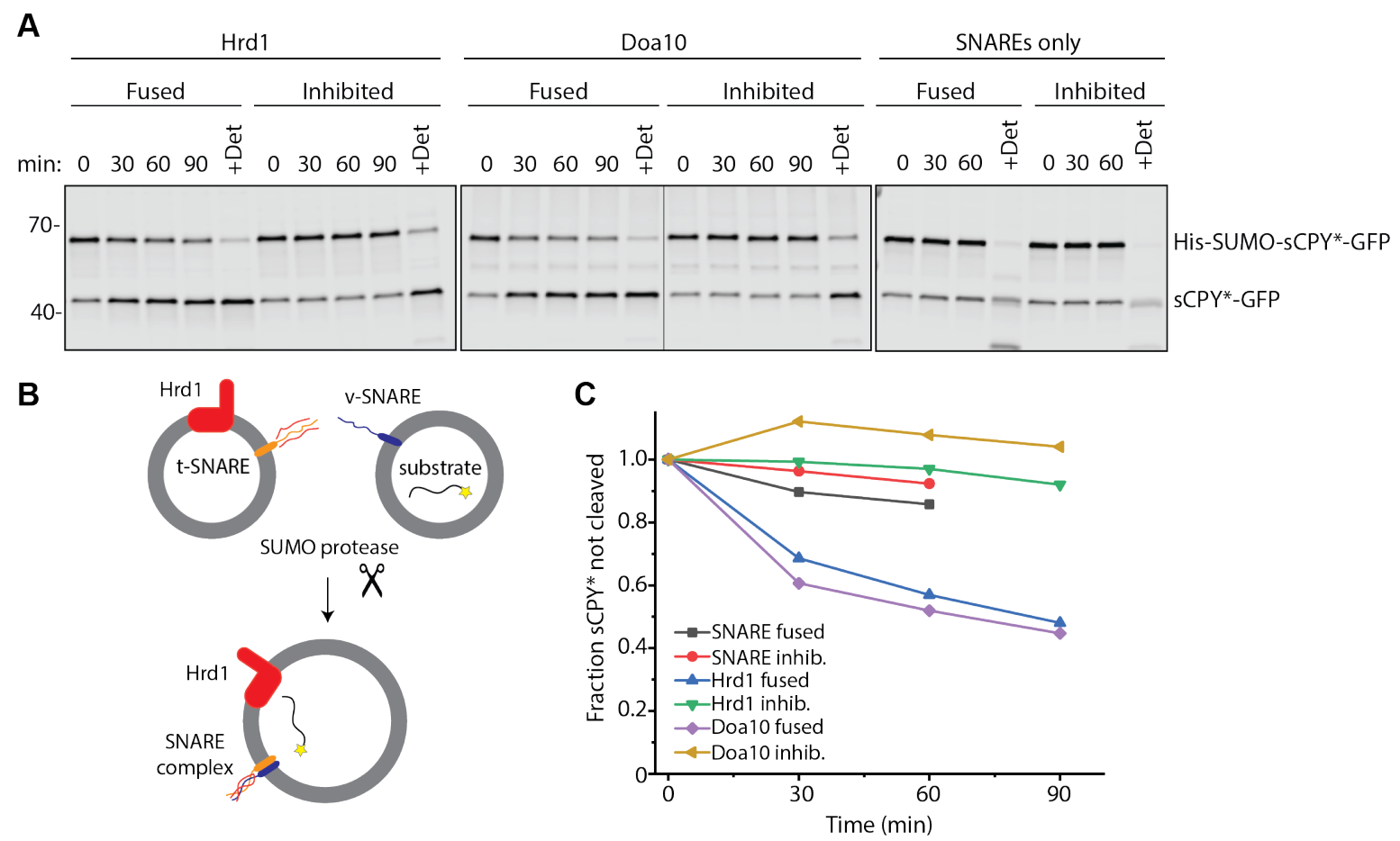

Figure 3.21: Leakage occurs during fusion and is dependent on protein:lipid ratio

A: His-SUMO-sCPY*_GFP/Syb liposomes were fused with $\mathrm{Hrd} 1 / \Delta$ N53, Doa10/ $\Delta$ N53, or $\Delta$ N53-only liposomes in the presence of $2 \mu \mathrm{M}$ Ulp1. Hrd1, Doa10 and $\Delta$ N53 were reconstituted at protein:lipid ratios of 1:1000. Timepoints were taken into SDS sample buffer and analyzed for cleavage by SDS-PAGE. B: Scheme of leakage assay: fusion in the presence of SUMO protease (Ulp1). C: Quantification of His-SUMO-sCPY*-GFP (top band) SNAREs only: Fusion of substrate liposomes with $\Delta$ N53-only liposomes. 


\subsection{6 $\operatorname{PrA} *$ is a Hrd1-substrate in detergent}

So far, all of the retrotranslocation experiments utilized variations of the canonical ERAD-L substrate CPY*. In order to not limit the investigation to only one model substrate, I asked if another ERAD-L substrate could be retrotranslocated by Hrd1 in the fusion system. I chose the substrate $\operatorname{PrA}^{*}$, a misfolded vacuolar protease that is degraded in a Hrd1-dependent manner in S. cerevisiae, requiring the same ERAD-L components as CPY* (Finger et al., 1993; Spear and Ng, 2005; Kanehara et al., 2010). $\mathrm{PrA}^{*}$ has never been investigated in a reconstituted system. Therefore, I expressed it in $S$. cerevisiae and purified it with a C-terminal His ${ }_{14}$ tag. PrA*, like CPY*, was aggregation-prone and was purified in $2 \mathrm{M}$ urea to maintain solubility. After sortaselabeling, $\operatorname{PrA}^{*}$ eluted as two peaks in size exclusion chromatography (Figure 3.22A), with peak 1 being close to the exclusion limit of the column. When mixed with Hrd1 and ubiquitination machinery in detergent, peak 2 was ubiquitinated as efficiently as

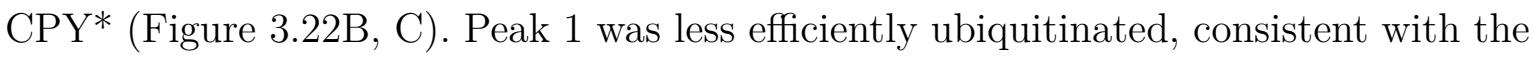
fact that it eluted close to the void volume and was likely in an aggregated state. PrA* contains two glycosylation sites and appeared to be purified as a mixture of nonglycosylated and glycosylated forms (Figure 3.22B). However, this would need to be confirmed by treatment with endoglycosidase H. Interestingly, peak 2 contained mainly the fully glycosylated form, but all forms of $\operatorname{PrA}^{*}$ were ubiquitinated independent of the glycosylation status (Figure 3.22B). Peak 2 was used in subsequent retrotranslocation experiments. 


\section{A}
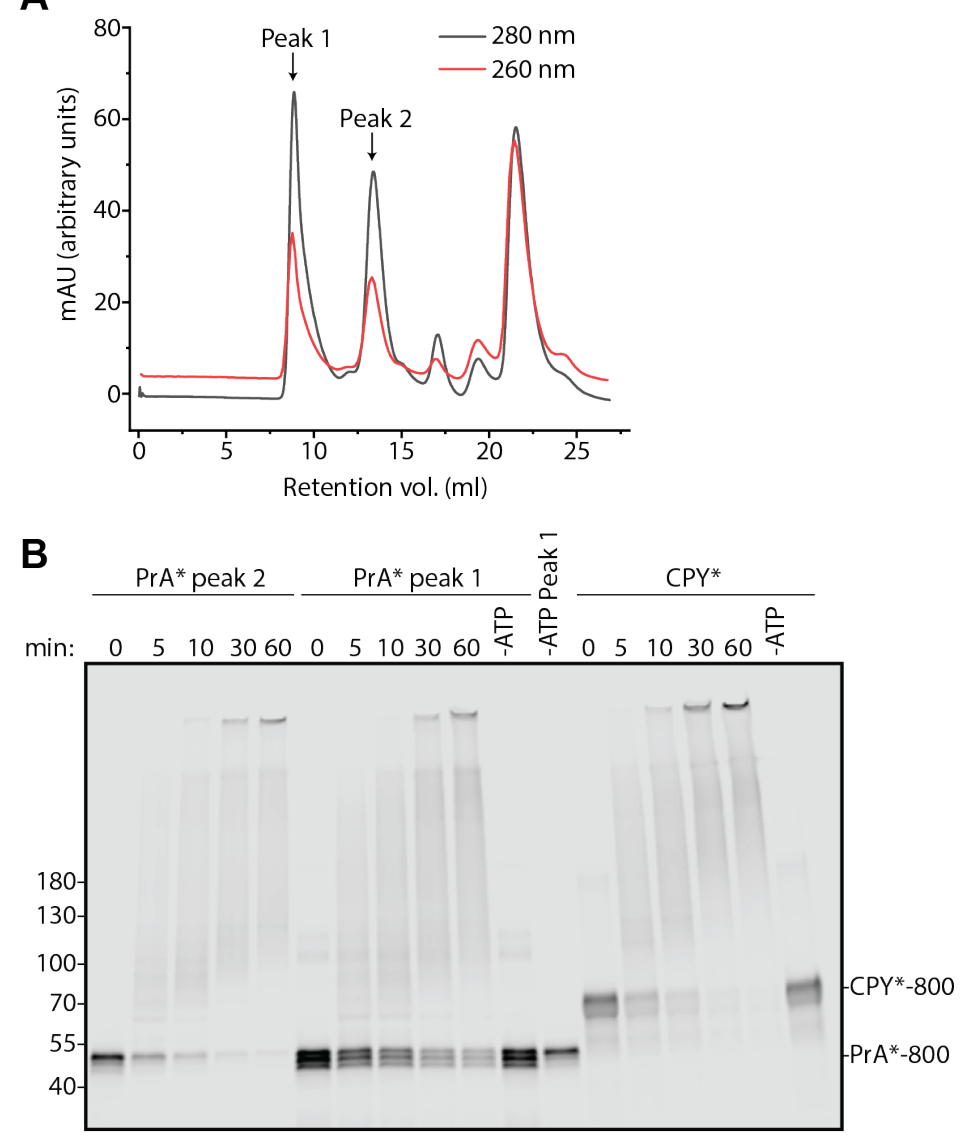

C

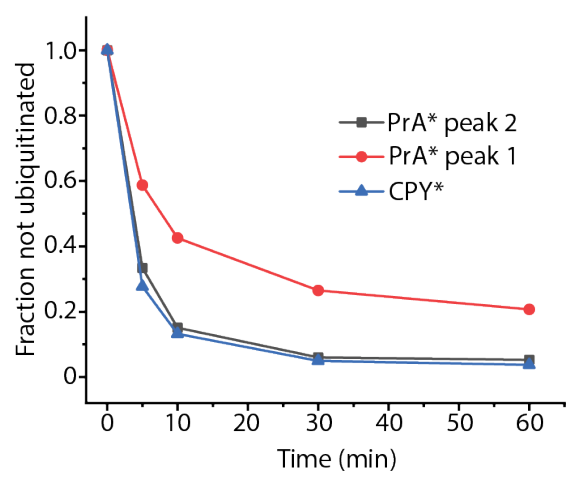

Figure 3.22: $\operatorname{PrA} A^{*}$ is efficiently ubiquitinated by $\mathrm{Hrd1}$ in detergent A: Size exclusion chromatography of PrA* in 2 M urea on a Superdex 200 10/300 GL column, after sortase labeling. Peak 1 eluted close to the void volume of $8 \mathrm{ml}$. B: Ubiquitination time-course of PrA* and $\mathrm{CPY}^{*}$ by Hrd1 in detergent (120 $\mu \mathrm{M}$ DMNG). C: Quantification of fraction $\operatorname{PrA}^{*} / \mathrm{CPY}^{*}$ ubiquitination, normalized to the $0 \mathrm{~min}$ timepoint.

\subsubsection{PrA* does not get retrotranslocated by Hrd1 in the fusion system}

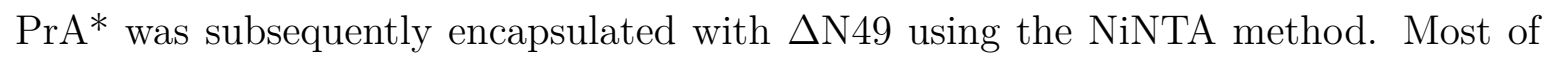
the PrA* floated in the top fractions, indicating that encapsulation efficiency was high (Figure 3.23A). $88 \%$ of $\mathrm{PrA}^{*}$ in the floated fraction was encapsulated, as determined by protease protection (Figure 3.23B). 
A

imidazole

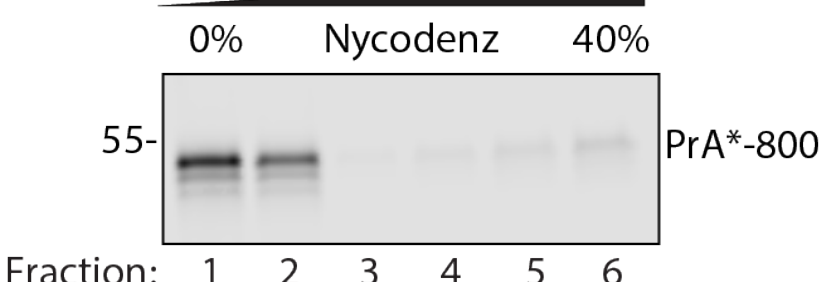

B

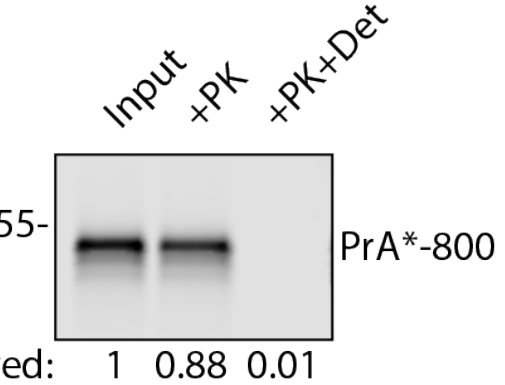

Figure 3.23: Encapsulation of $\operatorname{PrA}$ * using the NiNTA method

A: PrA*-His ${ }_{14}$ was encapsulated into liposomes with $\Delta$ N49 using the NiNTA method and was floated in a Nycodenz gradient with imidazole. Fractions were collected and analyzed by SDS-PAGE and fluorescence scanning. B: Liposomes were harvested from the gradient and subjected to protease protection with proteinase K. Det: Triton X-100.

Upon fusion with Hrd1/Syb liposomes and addition of ubiquitination machinery, approximately $12 \%$ of $\mathrm{PrA}^{*}$ was ubiquitinated (Figure $3.24 \mathrm{~A}, \mathrm{~B}$ ). This corresponded with the fraction of $\mathrm{PrA}^{*}$ that was not protected from proteinase $\mathrm{K}$, indicating that the ubiquitinated fraction could have originated exclusively from the outside-bound PrA* $^{*}$. Indeed, this was most likely the case, as the inhibited fusion control showed the same efficiency of $\operatorname{PrA}^{*}$ ubiquitination (Figure 3.24B). Therefore, Hrd1 did not retrotranslocate PrA*. 

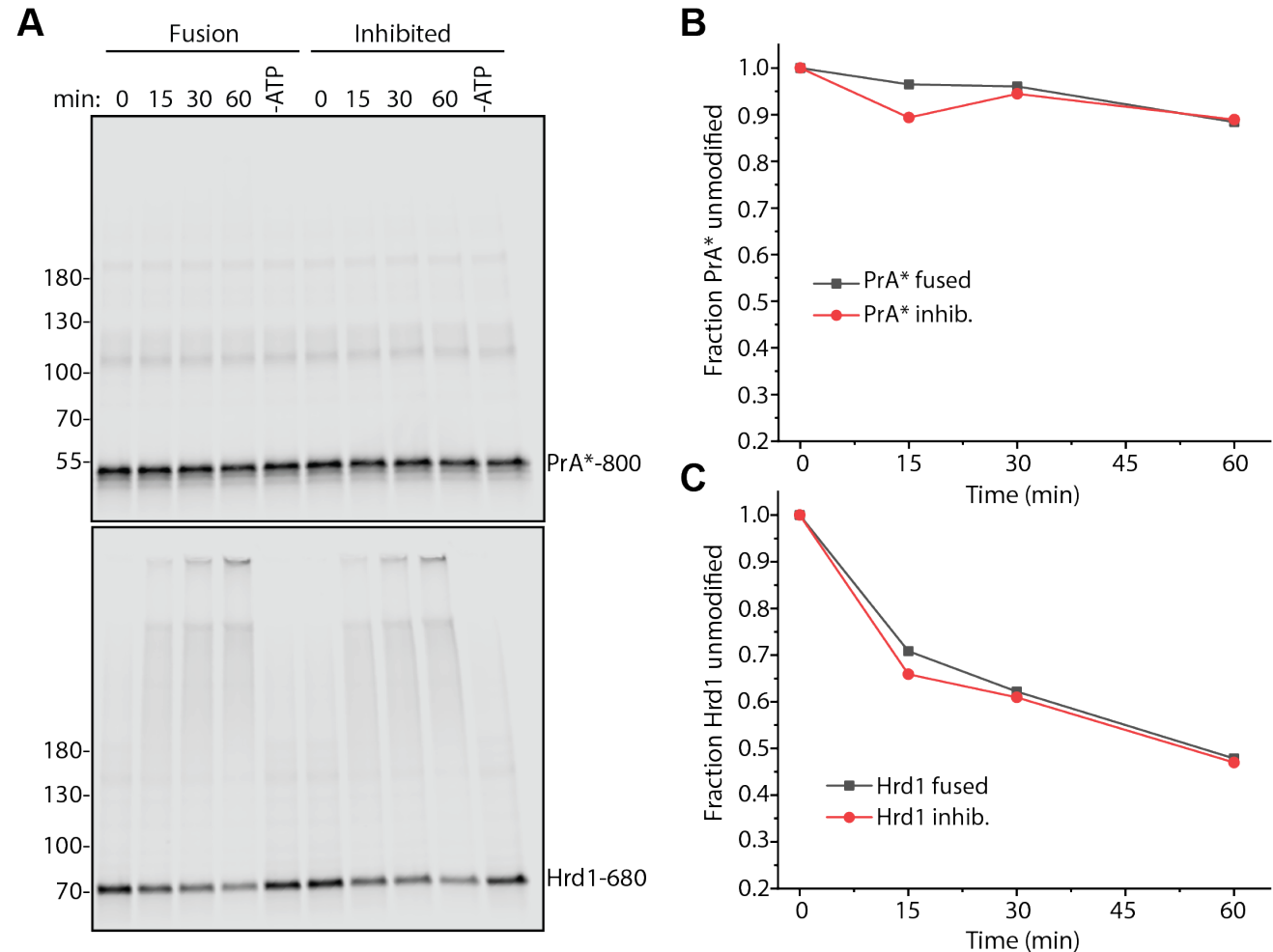

Figure 3.24: $\operatorname{PrA} A^{*}$ does not get retrotranslocated by Hrd1

A: $\operatorname{PrA}^{*}$-His $_{14} / \Delta \mathrm{N} 49$ and Hrd/Syb liposomes were fused and mixed with ubiquitination machinery. ATP was added to start the reaction. Where indicated, fusion was inhibited by incubating $\operatorname{PrA}^{*}-\mathrm{H}_{14} / \Delta \mathrm{N} 49$ liposomes with $\mathrm{Syb}_{\text {sol }}$. B: Quantification of ubiquitination of PrA* and Hrd1 $(\mathbf{C})$, normalized to the 0 min timepoint.

\subsubsection{Retrotranslocation of membrane-anchored ERAD-L substrates}

As a control for the fusion system, I attempted to use CPY*-TM and CPY*-TM2, which contain a C-terminal transmembrane domain, allowing them to be reconstituted without the NiNTA method (Baldridge and Rapoport, 2016). After insertion into liposomes with a random orientation, the luminally-oriented substrate is relabeled to a different fluorescent dye using the sortase technique (Baldridge and Rapoport, 2016). Unfortunately, I was not able to reconstitute CPY*-TM or TM2 with CPY* facing the lumen. This was assessed with protease protection with Lys-C protease, which cannot cleave the C-terminus of the transmembrane domain. Despite my efforts to replicate the protocol from Baldridge and Rapoport (2016), CPY* was always completely accessible to Lys-C and did not float efficiently in Nycodenz flotation assays (data not shown). This behavior was persistent across a range of salt, urea and glycerol concentrations, 
as well as various detergent removal methods. It appeared that CPY*-TM substrates were aggregating instead of inserting with their TM into liposomes.

\subsection{Bypassing fusion by sequential coreconstitution of Hrd1 and substrate}

In order to further characterize the lack of efficient retrotranslocation in the fusion system, I decided to bypass the fusion step by reconstituting Hrd1 directly into liposomes containing encapsulated CPY*. If retrotranslocation were to be observed using this coreconstitution approach, this would point to the fusion being the bottleneck in the system. Of course, the coreconstitution approach has disadvantages, namely the possible detergent-mediated interaction between CPY* and Hrd1 (as discussed in section 3.1.1). However, the purpose of this approach was to determine if, upstream of fusion, Hrd1 was capable of retrotranslocating encapsulated CPY*. Even if this were due to detergent-mediated interactions, it would provide a proof of concept that retrotranslocation of $\mathrm{CPY}^{*}$ can be observed in certain circumstances..

To this end, CPY*-H14 was encapsulated using the NiNTA method and these liposomes were then partially solubilized with limiting amounts of DMNG ( $R$ value of 0.35, Figure 3.25A). Hrd1 was added to the partially solubilized liposomes, with the expectation that encapsulated $\mathrm{CPY}^{*}$ would not leak to the outside. Upon detergent removal, Hrd1 inserted into $\mathrm{CPY}^{*}$ liposomes, judged by its coflotation with CPY* (Figure 3.25B). Additionally, no decrease in $\mathrm{CPY}^{*}$ encapsulation was detected by proteinase $\mathrm{K}$ protection after Hrd1 addition (Figure 3.25C). In fact, slightly more CPY* was protected after Hrd1 addition. 
A
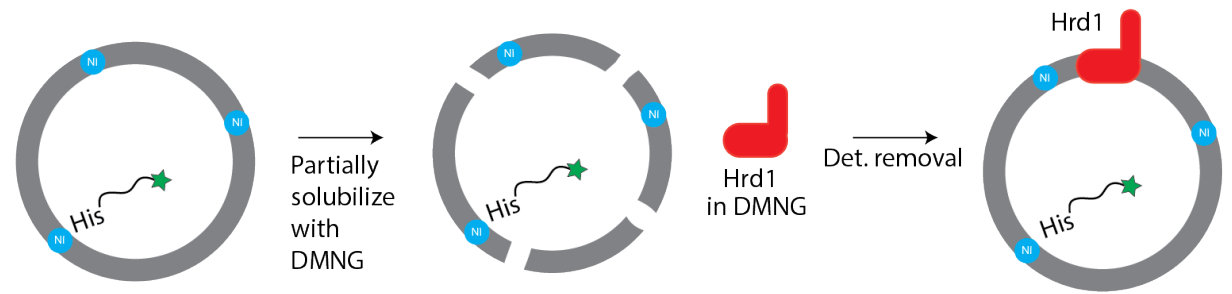

B

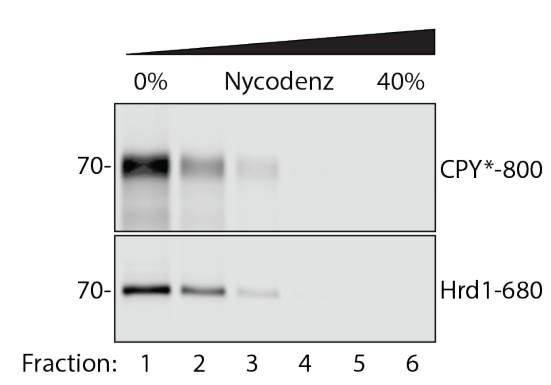

C

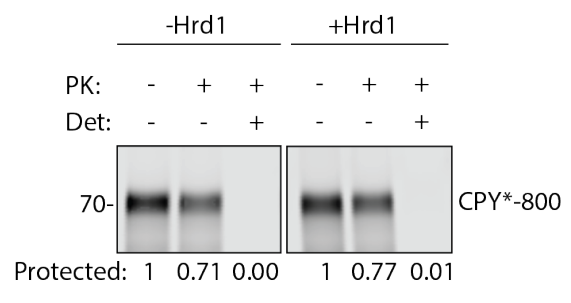

Figure 3.25: Sequential coreconstitution of Hrd1 into CPY* liposomes

A: Scheme of reconstitution strategy. Liposomes containing encapsulated CPY*-H14 were partially solubilized with DMNG at an $\mathrm{R}$ value of 0.35 and $\operatorname{Hrd} 1$ was added. Upon removal of DMNG, Hrd1 inserted into CPY* liposomes. B: Nycodenz flotation of Hrd1/CPY* liposomes. C: Protease protection of $\mathrm{CPY}^{*}$ with proteinase $\mathrm{K}$ before and after Hrd1 addition. Det: Triton X-100.

Upon addition of ubiquitination machinery and ATP, approximately 30\% of CPY* was ubiquitinated by Hrd1 (Figure 3.26A, B). Because $23 \%$ of CPY* was accessible to proteinase $\mathrm{K}$ (Figure 3.25), this meant that up to $7 \%$ of $\mathrm{CPY}^{*}$ was possibly retrotranslocated. However, this was quite inefficient and did not approach retrotranslocation efficiencies observed in the Baldridge study. Therefore, bypassing fusion by detergent-mediated Hrd1 incorporation did not significantly increase the retrotranslocation efficiency of $\mathrm{CPY}^{*}$. 
A

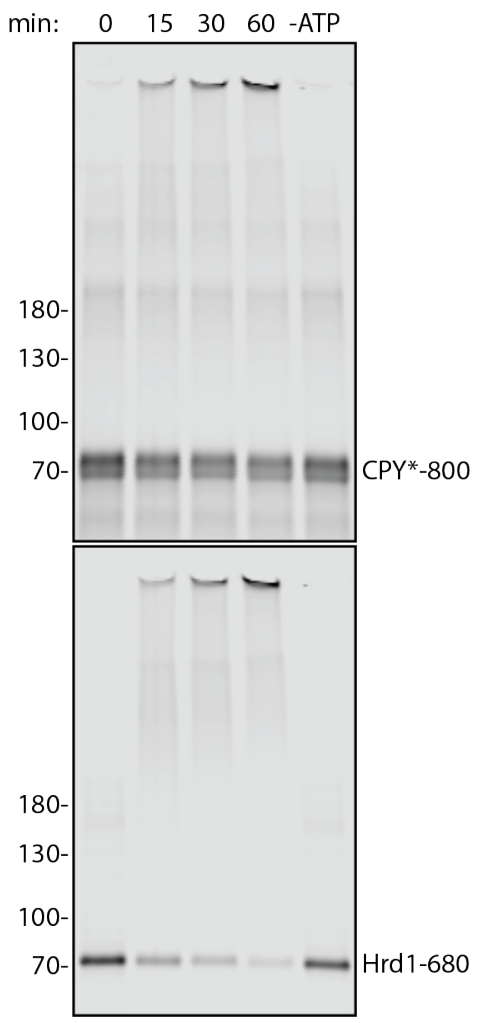

B

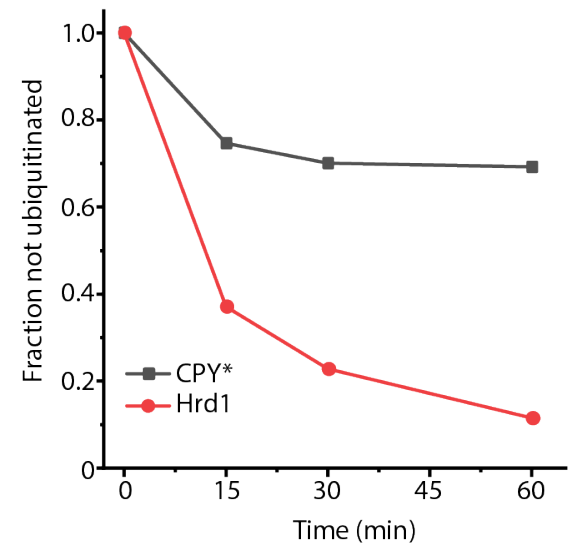

Figure 3.26: $\mathrm{CPY}^{*}$ ubiquitination after sequential Hrd1 coreconstitution A: Hrd1/CPY* liposomes made by sequential Hrd1 coreconstitution were mixed with ubiquitination machinery and ATP. The -ATP control was taken after 60 min. B: Quantification of $\mathrm{CPY}^{*}$ and Hrd1 ubiquitination, normalized to 0 min timepoint.

\subsection{Problems with the retrotranslocation experiments}

\subsubsection{Aggregation of substrates}

There are a variety of factors that could contribute to the lack of retrotranslocation observed in the fusion system and in the sequential coreconstitution. Because fusion efficiency and Hrd1 activity did not appear to be the limiting factors, the problem most likely lied in the substrate itself. The ERAD-L substrates CPY* and $\mathrm{PrA}^{*}$ are aggregation-prone. In their purified state, they aggregate within a few hours without the presence of detergent or urea (personal observation). In the cell, misfolded proteins are kept in a soluble state in the ER lumen by chaperones. Abrogation of the chaperone system causes $\mathrm{CPY}^{*}$ and other luminal substrates to aggregate and inhibits their degradation by ERAD (Nishikawa et al., 2001). 
Upon encapsulation into liposomes, $\mathrm{CPY}^{*}$ is in close proximity to the membrane, and its exposed hydrophobic regions may partially insert into the lipid bilayer. Both aggregation and sticking to the membrane would likely block retrotranslocation. As an indirect readout of aggregation, I tested the ubiquitination of outside-bound CPY* after reconstitution into liposomes (Figure 3.27A). As shown in Figure 3.27B, after the first step of the NiNTA encapsulation protocol, $27 \%$ of CPY* was protected from proteinase $\mathrm{K}$, meaning that $73 \%$ of $\mathrm{CPY}^{*}$ was located on the outside of the liposomes. However, when fused with Hrd1 liposomes and incubated with ubiquitination machinery, only $30 \%$ of the CPY* was ubiquitinated (Figure 3.27C, D). Thus, despite the fact that the majority of $\mathrm{CPY}^{*}$ was on the outside of the liposomes and accessible to Hrd1 after fusion, it was still inefficiently ubiquitinated. In contrast, when CPY* was diluted from urea and added directly to the outside of Hrd1 liposomes, it was nearly completely ubiquitinated (see section 3.9.1 and Figure 3.34), indicating that the reconstitution into liposomes caused aggregation of $\mathrm{CPY}^{*}$.

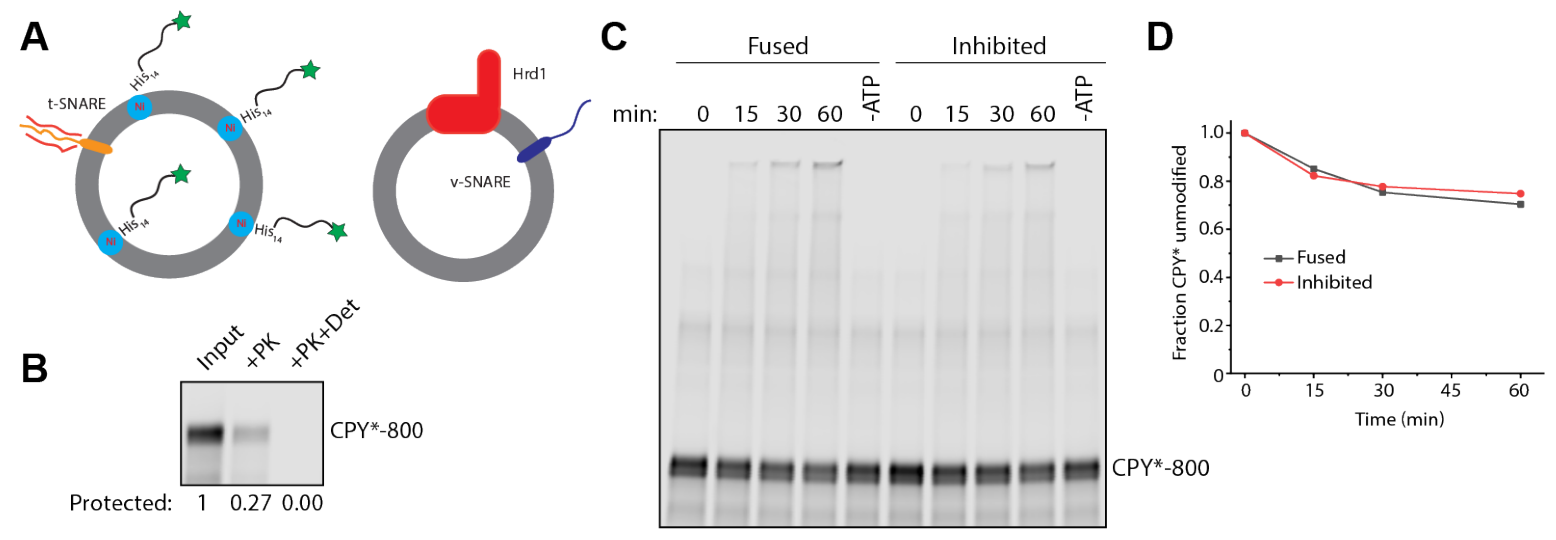

Figure 3.27: $\mathrm{CPY}^{*}$ reconstituted on outside of liposomes is inefficiently ubiquitinated by Hrd1 after fusion

A: CPY*-H14 was encapsulated with $\Delta$ N49 using the NiNTA method. B: Unfloated liposomes were analyzed by proteinase $\mathrm{K}$ protection. Fraction protected is indicated below the lanes. C: Unfloated $\mathrm{CPY}^{*}-\mathrm{H} 14 / \Delta \mathrm{N} 49$ liposomes were fused with Hrd1 liposomes and incubated with ubiquitination machinery. Timepoints were analyzed by SDS-PAGE and fluorescence scanning. D: Quantification of ubiquitination in (C). About $30 \%$ of $\mathrm{CPY}^{*}$ was ubiquitinated, independent of fusion.

\subsubsection{Improving solubility of encapsulated CPY* with urea}

In order to increase the solubility of encapsulated CPY*, I treated the liposomes with increasing concentrations of urea during the Nycodenz/imidazole flotation step. Urea is a denaturing agent commonly used to unfold or stabilize membrane proteins and protein 
aggregates during purification. Because it is a small nonpolar molecule, it is membrane permeable and diffuses through lipid bilayers (Lippe, 1969). The rationale was that urea would diffuse into the lumen and solubilize aggregated CPY*. Because high urea concentrations would likely interfere with subsequent fusion and ubiquitination reactions, $\mathrm{CPY}^{*}$ liposomes were diluted by a factor of 10 into the fusion reaction. The idea was that there would be a time window before aggregation, where CPY* could interact with Hrd1 and be retrotranslocated.

In the standard NiNTA encapsulation protocol, I included $1 \mathrm{M}$ urea in the top layers of the Nycodenz gradient. To modify this, increasing concentrations of urea starting from 1.5 M were added to the top layers of the Nycodenz gradient. Upon flotation, CPY* liposomes were fused with Hrd1 liposomes and incubated with ubiquitination machinery. Unfortunately, I did not observe an increase in $\mathrm{CPY}^{*}$ ubiquitination efficiency from 1.5 to $3 \mathrm{M}$ urea (Figure 3.28A, B). Ubiquitination efficiency was about $15 \%$ in all cases, corresponding again with the fraction of $\mathrm{CPY}^{*}$ which was unprotected from proteinase K (compare Figure 3.28B to Figure 3.16B). Dilution of CPY* liposomes into the reaction introduced up to $180 \mathrm{mM}$ urea. Nevertheless, Hrd1 autoubiquitination was not impaired at these urea concentrations (Figure 3.28C). 

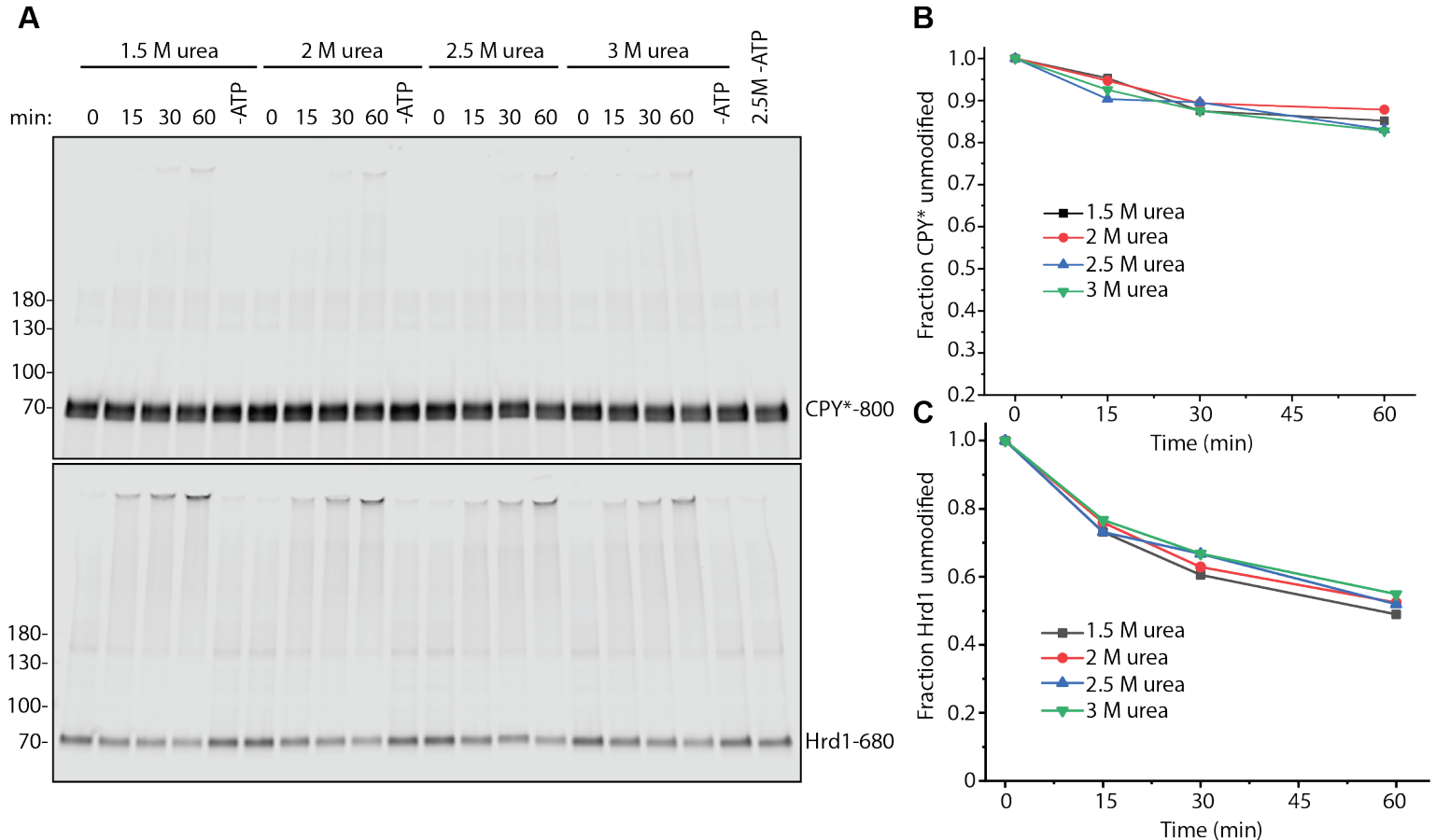

Figure 3.28: Urea titration of $\mathrm{CPY}^{*} / \Delta \mathrm{N} 49$ liposomes

A: Liposomes containing encapsulated H14-CPY* with $\Delta \mathrm{N} 49$ were floated in a $\mathrm{Ny}-$ codenz/imidazole step gradient with increasing concentrations of urea in the top layers. The liposomes were harvested from the top of the gradient, diluted, fused with Hrd1/Syb liposomes, and incubated with ubiquitination machinery. Timepoints were analyzed by SDS-PAGE and fluorescence scanning. B: Quantification of ubiquitination of $\mathrm{CPY}^{*}$ and $\operatorname{Hrd1}(\mathbf{C})$, normalized to the 0 min timepoint. 


\subsection{Electrophysiological investigation of Hrd1 retrotranslocase activity}

The fusion system posed difficulties in investigating retrotranslocation by Hrd1, primarily due to aggregation problems with the substrate. As an alternative technique to study Hrd1 retrotranslocation, I collaborated with Dr. Niels Denkert (University Medical Center, University of Göttingen and Max Planck Institute for Biophysical Chemistry) and Prof. Michael Meinecke (University Medical Center, University of Göttingen) to reconstitute Hrd1 into planar lipid bilayers (PLBs) for electrophysiological characterization. The PLB technique has been used to extensively characterize a variety of translocation channels, such as mitochondrial Tim23 and Tom40, eukaryotic Sec61, bacterial SecY, and Toc75 from chloroplasts (Truscott et al., 2001; Meinecke et al., 2006; Hill et al., 1998; Wirth et al., 2003; Saparov et al., 2007; Hinnah et al., 2002). The principle of the technique is that a bilayer is formed in a small aperture flanked by two aqueous chambers which can be manipulated by the experimenter (Harsman et al., 2011). It has been widely demonstrated that protein translocation channels can form water-filled pores that have the capacity to conduct ions in a voltagedependent manner (Harsman et al., 2011). Using ion conductance as a readout, the PLB technique allows for the investigation of pore diameter, substrate interactions, and the mechanism of channel formation with single-molecule resolution.

\subsubsection{Reconstitution of Hrd1 into liposomes for PLB experiments}

We hypothesized that Hrd1 would form a water-filled channel in its autoubiquitinated state and would thus be amenable to characterization with the PLB technique. In order to insert a protein of interest into the PLB, it is first reconstituted into liposomes. Proteoliposomes are then added to the PLB chamber, and liposomes that contain active channels undergo osmotic swelling and fuse with the PLB (Cohen et al., 1980; Niles et al., 1989). To this end, I reconstituted Hrd1 into liposomes with DMNG, as previously described (Stein et al., 2014). Hrd1 was efficiently incorporated into liposomes, as it floated in a Nycodenz step gradient (Figure 3.29A). Treatment of the liposomes with TEV protease showed that Hrd1 was incorporated in the correct orientation, with its C-terminus and RING domain facing the outside (Figure 3.29B). Hrd1 liposomes autoubiquitinated efficiently when incubated with ubiquitination machinery, with approximately $80 \%$ ubiquitinated after 1 hour (Figure 3.29C). We proceeded with these Hrd1 liposomes in PLB experiments. 

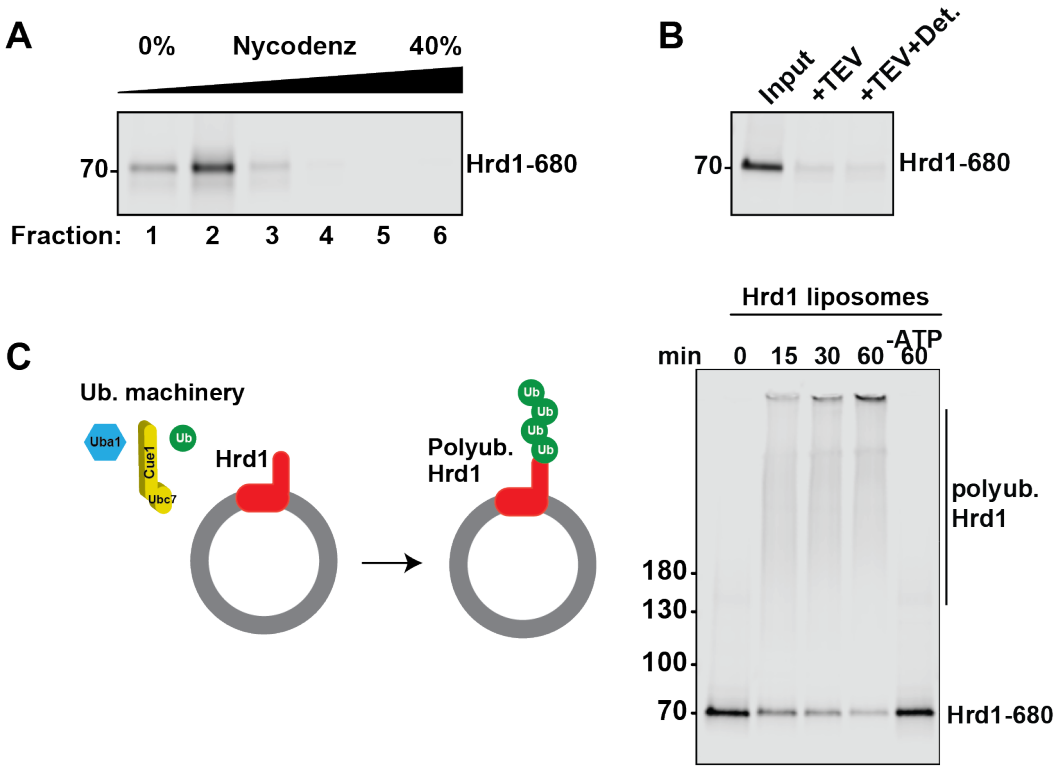

Figure 3.29: Hrd1 reconstitution into liposomes for planar lipid bilayer experiments

A: Hrd1 was reconstituted into liposomes with DMNG and floated in a Nycodenz step gradient. Hrd1 was efficiently incorporated into liposomes, as all of it floated at the top of the gradient. B: To assess orientation, Hrd1 liposomes were treated with TEV protease with or without DMNG (Det.). TEV protease cleaves off the sortase tag and fluorescent dye on the C-terminus of Hrd1. C: Hrd1 liposomes were incubated with ubiquitination machinery and ATP was added to start the reaction. Timepoints were analyzed by SDS-PAGE and fluorescence scanning.

\subsubsection{Hrd1 forms a dormant channel upon autoubiquitination}

All electrophysiology experiments described in the following sections (3.8.2-3.8.5, and section 3.10.2, Figure 3.44) were performed by Dr. Niels Denkert (University Medical Center, University of Göttingen and Max Planck Institute for Biophysical Chemistry), who also kindly provided the figures. When nonubiquitinated Hrd1 liposomes were added to PLBs, no current was detected at holding potentials of +40 or $-40 \mathrm{mV}$ (Figure 3.30A). In fact, we never observed fusion of nonubiquitinated Hrd1 liposomes with PLBs (quantification in Figure 3.30C). Because fusion by osmotic swelling depends on the presence of active channels (Niles et al., 1989), this indicates that nonubiquitinated Hrd1 likely does not form a channel. Strikingly, when Hrd1 liposomes were incubated with ubiquitination machinery and ATP, we observed fusions that led to small currents and sporadic gating events at different holding potentials (Figure 3.30B, C). The gating events showed small conductance changes, indicative of a small pore or dormant channel. Importantly, no fusions were observed when Hrd1 liposomes were incubated 
with ubiquitination machinery lacking ATP (Figure 3.30C).
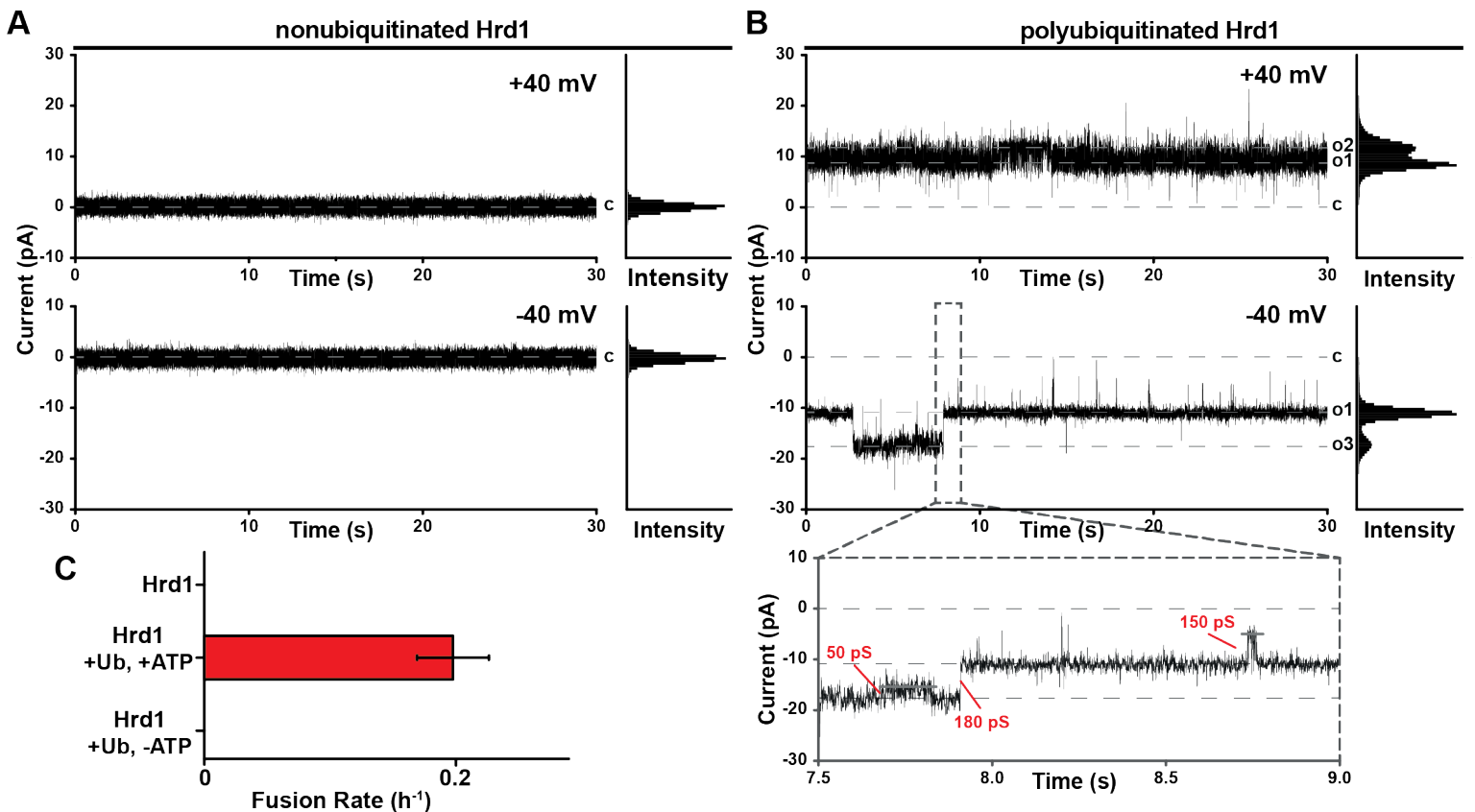

Figure 3.30: Hrd1 forms an ion-conducting channel only when polyubiquitinated

Current traces of nonubiquitinated $(\mathbf{A})$ and polyubiquitinated Hrd1 liposomes (B) at holding potentials of +40 and $-40 \mathrm{mV}$. Dashed lines indicate the closed states and different open states (c, o1, o2). Inset in B: expanded view of indicated region with conductance changes of independent gating events shown. C: Quantification of fusion rate per liposome preparation. Hrd1 refers to nonubiquitinated Hrd1. +Ub: Hrd1 liposomes incubated with ubiquitination machinery. $n=3$ independent experiments, error bars indicate SEM. Experiments and analysis were performed by Dr. Niels Denkert (University Medical Center, University of Göttingen, Max Planck Institute for Biophysical Chemistry).

\subsubsection{CPY* stimulates Hrd1 channel activity}

Although we observed small currents and sporadic gating events in Figure 3.30, the conductance changes observed were about 40-50 pS on average, with very few gating events observed above $200 \mathrm{pS}$ (Figure 3.31D). As a comparison, the S. cerevisiae Sec61 translocon was shown to undergo frequent gating events with conductance changes ranging between 200-700 pS, from which pore sizes of up to 1-6 nm were calculated (Wirth et al., 2003). We reasoned that if Hrd1 forms a retrotranslocon, it should also undergo gating events with similar conductance changes. We postulated that the channel may have to be activated by substrate. To this end, we fused ubiquitinated Hrd1 channels to the PLB and added CPY* to both sides of the membrane. Remarkably, 
addition of $\mathrm{CPY}^{*}$ stimulated considerably more frequent gating of Hrd1, with significantly greater conductance changes than observed without $\mathrm{CPY}^{*}$ (Figure 3.31A, C, D). The channel activation was specific to misfolded proteins, as WT CPY added at a two-fold greater concentration did not induce any additional channel gating or conductance changes (Figure 3.31B, C, D). CPY* added to the PLB alone did not result in any detectable current, indicating that the channel stimulation was specific for the $\mathrm{CPY}^{*}$ interaction with Hrd1 and not due to nonspecific effects of a misfolded protein interacting with the bilayer (Figure 3.31C).

Upon $\mathrm{CPY}^{*}$ stimulation, Hrd1 gating events with conductance changes greater than $100 \mathrm{pS}$ were significantly increased, with conductance changes in the range of 200800 pS observed (Figure 3.31C, D). These conductance states were similar to those previously observed for Sec61 (Wirth et al., 2003), indicating that Hrd1 can open to similar pore diameters upon interaction with substrate. Thus, CPY* stimulated Hrd1 channel activity, causing it to gate more frequently and leading to expansion of the pore. 


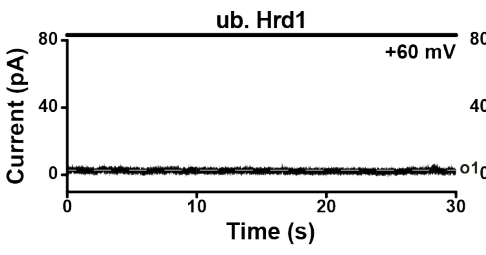

B
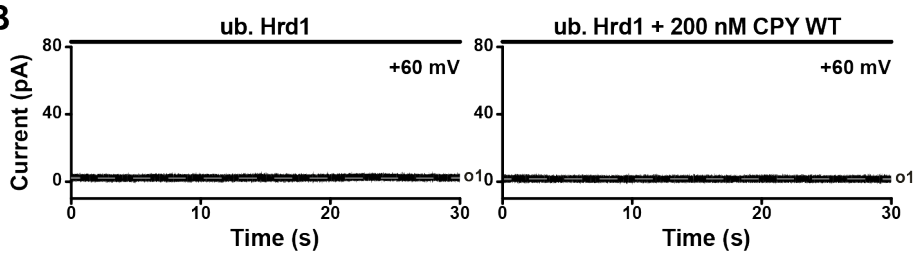

ub. Hrd1 + 100 nM CPY*

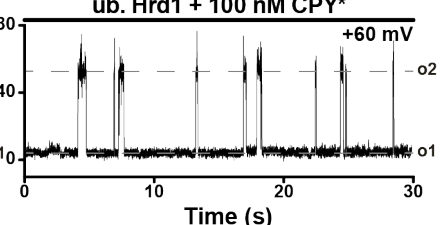

ub. Hrd1 + 200 nM CPY WT

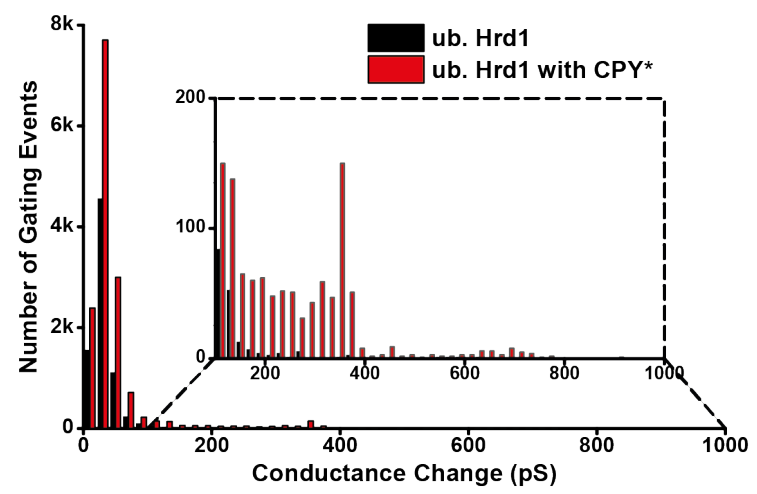

C

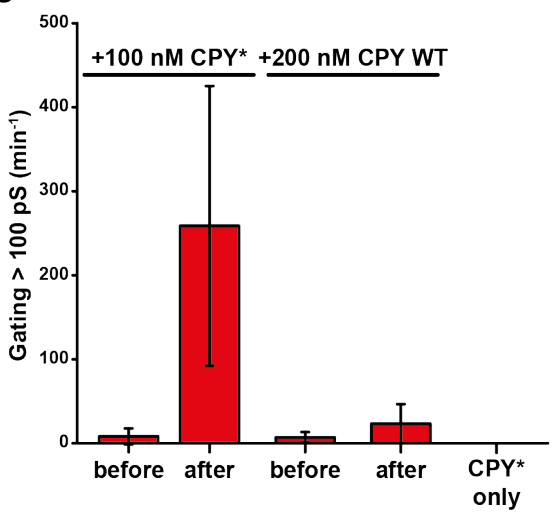

Figure 3.31: $\mathrm{CPY}^{*}$ addition to inserted Hrd1 channels stimulates gating and opening of the channel

Current traces of ubiquitinated Hrd1 with and without CPY* (A) or CPY WT (B) at holding potentials of +60 and $-60 \mathrm{mV}$. Dashed lines indicate two different open states of the channel (o1, o2). C: Quantification of gating events with conductance changes greater than $100 \mathrm{pS}$, before and after $\mathrm{CPY}^{*}$ or CPY WT addition. $\mathrm{n}=3$ independent experiments, error bars indicate SEM. D: Conductance histogram of gating events from ubiquitinated Hrd1 channels with or without addition of $100 \mathrm{nM} \mathrm{CPY}^{*}$ to the chamber. Inset shows a zoom plot of a region with higher conductance states for better visualization. Experiments and analysis were performed by Dr. Niels Denkert (University Medical Center, University of Göttingen and Max Planck Institute for Biophysical Chemistry).

\subsubsection{Deubiquitination of Hrd1 closes the channel}

Ubiquitination is a reversible post-translational modification. We therefore asked if deubiquitination of Hrd1 closes the channel. This is important, as having a constitutively open retrotranslocation channel in the ER would likely be detrimental to the cell. To test this hypothesis, we fused ubiquitinated Hrd1 liposomes to the PLB and added the catalytic domain of the human deubiquitinase Usp2 (Renatus et al., 2006). In biochemical experiments with ubiquitinated Hrd1 in liposomes, addition of $1 \mu \mathrm{M}$ 
Usp2 efficiently deubiquitinated Hrd1 within 10 minutes (Figure 3.32C). Remarkably, addition the same concentration of Usp2 to the PLB containing ubiquitinated Hrd1 closed the channel, as current flow was abolished (Figure 3.32A). Channel closure was only observed when Usp2 was added to the cis side of the chamber and was complete within 5 minutes, whereas incubation with Usp2 on the trans side for 3x as long had no effect (Figure 3.32B). Because the orientation of Hrd1 in liposomes was unidirectional (Figure 3.29), with the RING domain facing the outside, polyubiquitin chains can only be attached to the cytoplasmic side of Hrd1. Therefore, this experiment demonstrated that Hrd1 maintained its orientation during fusion to the PLB, inserting unidirectionally, with the the cytoplasmic region containing the RING domain facing the cis side of the chamber. 
A
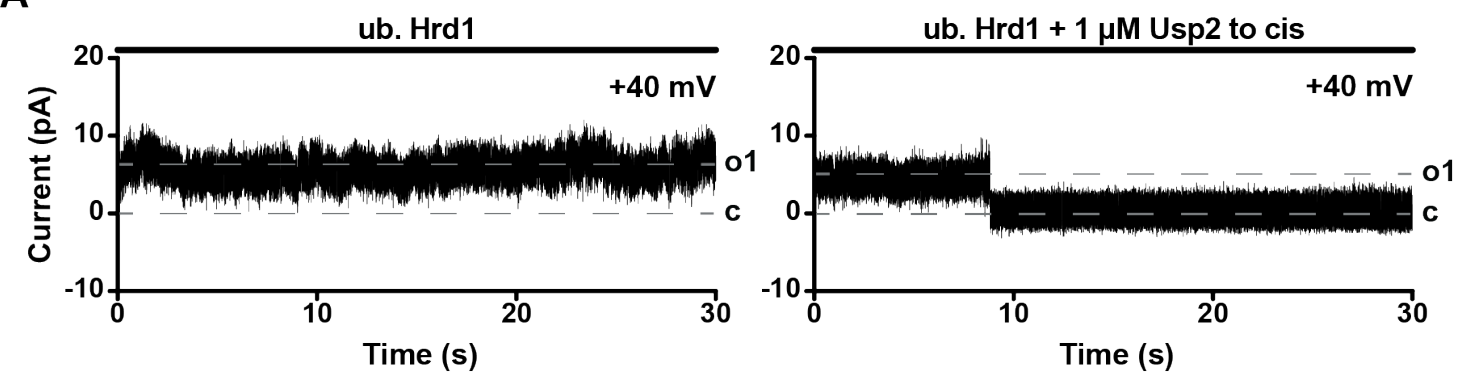

B

C
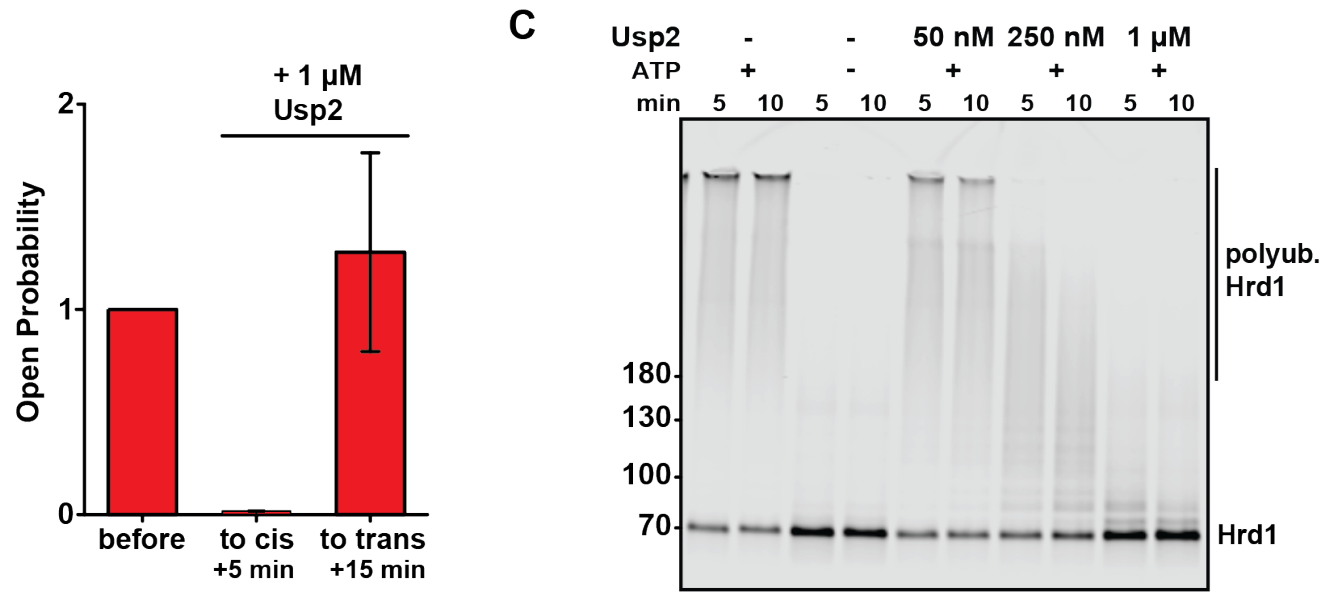

Figure 3.32: Deubiquitination of Hrd1 leads to channel closure

A: Current traces of ubiquitinated Hrd1 before (left) and after Usp2 addition (right) at a holding potential of $+40 \mathrm{mV}$. Dashed lines indicate the closed state and open states (c and o1). Note that addition of Usp2 abolishes current flow and closes the channel. B: Relative open probability of Hrd1 channels before Usp2 addition, and after side-specific addition of Usp2 to the cis and trans sides $(n=3$, error bars indicate SEM). C: Deubiquitination of polyubiquitinated Hrd1 in liposomes by Usp2. Hrd1 liposomes were immobilized on magnetic streptavidin beads and incubated with ubiquitination machinery. The liposomes were washed and eluted with biotin. Increasing concentrations of Usp2 were added and timepoints were analyzed by SDS-PAGE and fluorescence scanning. Experiments and analysis in (A) and (B) were performed by Dr. Niels Denkert (University Medical Center, University of Göttingen and Max Planck Institute for Biophysical Chemistry).

\subsubsection{CPY* stimulates Hrd1 channel activity only from the luminal side}

In previous experiments, we observed that CPY* stimulated Hrd1 channel activity when added to both sides of the chamber (Figure 3.31). Having established the orientation of Hrd1 in the PLB (Figure 3.32), we asked whether CPY* stimulation was side-specific. We hypothesized that $\mathrm{CPY}^{*}$ would stimulate channel activity only from 
the luminal side, as this is where luminal substrates first interact and insert into the channel. To this end, ubiquitinated Hrd1 liposomes were fused with the PLB and $\mathrm{CPY}^{*}$ was added either to the cytosolic (cis) or luminal (trans) side. Increased gating frequency was observed only when $\mathrm{CPY}^{*}$ was added to the luminal side of Hrd1 (Figure $3.33 \mathrm{~A}, \mathrm{~B})$. In this experiment, multiple Hrd1 channels inserted into the PLB, which explains why the gating frequency and conductance states appear more heterogenous than in Figure 3.31A. Therefore, substrate interaction with the luminal side of ubiquitinated Hrd1 stimulates channel gating and opening to larger pore sizes (see section 3.8.3, and Figure 3.31). This result suggests that Hrd1 alone can recognize substrates in the ER lumen, without the need for Hrd3, Der1 or Usa1. This may explain why the overexpression of Hrd1 makes these other components dispensable in vivo (Carvalho et al., 2010).

A

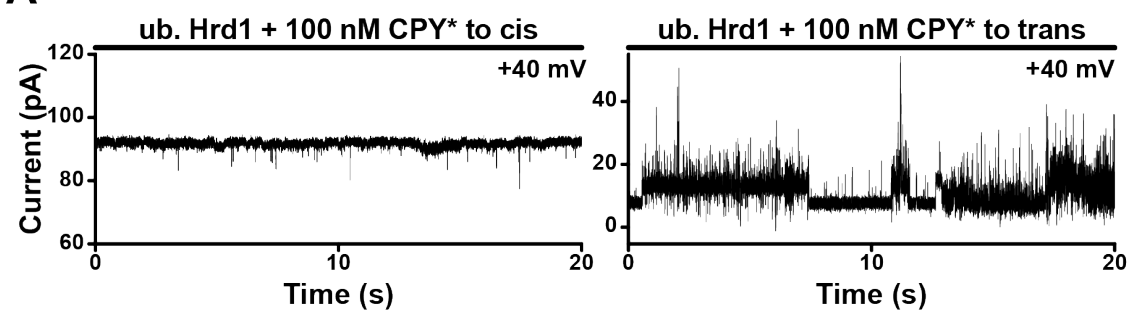

B

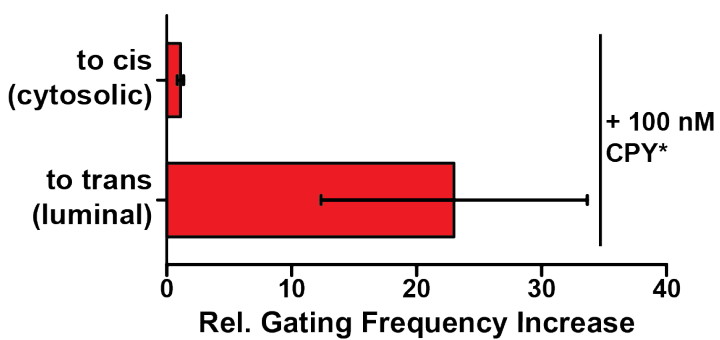

Figure 3.33: Addition of CPY* to the luminal side of ubiquitinated Hrd1 stimulates channel gating

Hrd1 liposomes were ubiquitinated and fused with PLBs. CPY* (100 nM) was added to either the cis (cytosolic) or trans (luminal) side of the chamber. A: Current traces of Hrd1 channels at $+40 \mathrm{mV}$ with side-specific $\mathrm{CPY}^{*}$ addition. Note the increased gating frequency upon addition of $\mathrm{CPY}^{*}$ to the trans (luminal) side. B: Quantification of relative gating frequency increase upon CPY* addition. Shown are means \pm SEM $(\mathrm{n}=3)$. Experiments and analysis were performed by Dr. Niels Denkert (University Medical Center, University of Göttingen and Max Planck Institute for Biophysical Chemistry). 


\subsection{Interaction of the cytoplasmic region of Hrd1 with substrates}

Having observed that CPY* binding stimulated Hrd1 channel activity in the PLB experiments (Figures 3.31, 3.33), I further investigated Hrd1 interaction with substrates biochemically. Previous studies showed an interaction between Hrd1 and CPY* in detergent (Stein et al., 2014; Baldridge and Rapoport, 2016), but this was likely mediated by the transmembrane domains of Hrd1, as shown in Stein et al. (2014). It remained unclear whether this interaction persisted with membrane-embedded Hrd1, where the TMs are occluded by the lipid bilayer. The relevant binding sites in retrotranslocation are the luminal and cytosolic sides of Hrd1, which contact the substrate before insertion into and after emergence from the channel. I took advantage of the liposome system, in which Hrd1 inserts into the bilayer unidirectionally with its cytoplasmic region facing the outside (Figure 3.29), to investigate the interaction between Hrd1 and substrates on the cytosolic side of the membrane.

\subsubsection{Hrd1 polyubiquitinates cytosolic ERAD-L substrates}

Because the RING domain of Hrd1 faces the outside, I asked whether Hrd1 could ubiquitinate CPY* when added to the outside of Hrd1 liposomes (referred to as cytosolic $\mathrm{CPY}^{*}$ from here on). Indeed, when cytosolic CPY* was added to Hrd1 liposomes and incubated with ubiquitination machinery, it was very efficiently ubiquitinated (Figure 3.34A, C). CPY WT was ubiquitinated less efficiently than CPY*, indicating that the Hrd1 cytoplasmic region can discriminate between misfolded and correctly-folded proteins, which was previously demonstrated for Hrd1 in detergent (Stein et al., 2014; Baldridge and Rapoport, 2016). However, a fraction of CPY WT was also ubiquitinated, but at a slower rate. The ERAD-L substrate PrA* (see section 3.5.6) was ubiquitinated with identical kinetics as CPY*, and PrA WT showed almost no ubiquitination (Figure 3.34B, C) The fact that cytosolic ERAD-L substrates were ubiquitinated by Hrd1 was surprising, because a similar experiment in the Baldridge study showed no ubiquitination of cytosolic $\mathrm{CPY}^{*}$ when it was added to CPY*-TM/Hrd1 liposomes (Baldridge and Rapoport, 2016). In that experiment, CPY*-TM most likely outcompeted cytosolic CPY* for Hrd1 binding. The result was also at odds with a previous study which found that cytosolic CPY* did not cofloat with Hrd1 liposomes in a flotation experiment (Stein et al., 2014). 

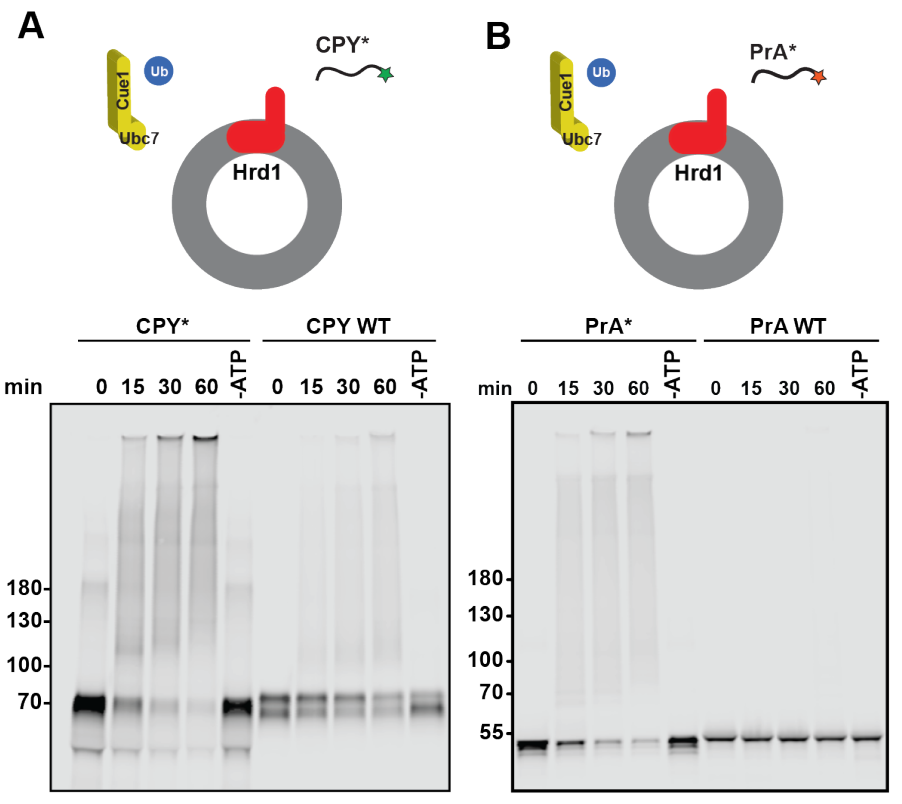

C

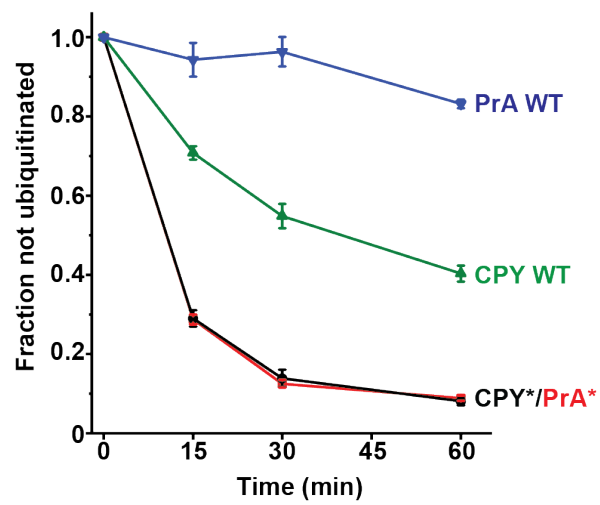

Figure 3.34: Hrd1 polyubiquitinates cytosolic CPY* and $\operatorname{PrA} *$ efficiently but not CPY WT and PrA WT

A, B: Hrd1 (200 nM) reconstituted in liposomes was incubated with the misfolded substrates $\mathrm{CPY}^{*}, \mathrm{PrA}^{*}$ or their corresponding WT versions $(100 \mathrm{nM})$. Ubiquitination machinery was added and timepoints were analyzed by SDS-PAGE and fluorescence scanning. C: Quantification of ubiquitination in (A) and (B) from three experiments (mean \pm standard deviation).

\subsubsection{Polyubiquitinated Hrd1 binds cytosolic CPY* and PrA*}

I inferred that the Hrd1 cytoplasmic region must bind to $\mathrm{CPY}^{*}$ and $\mathrm{PrA}^{*}$ in order to catalyze ubiquitination. Because $\mathrm{CPY}^{*}$ did not associate with nonubiquitinated Hrd1 liposomes in coflotation experiments (Stein et al., 2014), I hypothesized that binding might occur only after Hrd1 autoubiquitination. Autoubiquitination of Hrd1 likely results in a conformational change, which, in addition to opening the channel, may lead to the creation of a cytoplasmic binding site. To test this hypothesis, I immobilized Hrd1 liposomes onto streptavidin magnetic beads and ubiquitinated Hrd1 either in the presence or absence of ATP. The beads were washed to remove ubiquitination machinery and $\mathrm{CPY}^{*}$ was added. I observed almost complete $\mathrm{CPY}^{*}$ binding when Hrd1 was was ubiquitinated, and no binding compared to background binding to the beads when ATP was omitted (Figure 3.35, lanes 1, 2, 3 and 5). When Hrd1 was deubiquitinated with Usp2 before CPY* addition, CPY* did not bind (compare lanes 3 and 4), indicating that the conformational change induced by Hrd1 autoubiquitination was reversible. $\mathrm{CPY}^{*}$ and ubiquitinated Hrd1 were eluted with biotin, indicating that 
$\mathrm{CPY}^{*}$ binding was specific for Hrd1 and not due to nonspecific binding to the beads upon Hrd1 ubiquitination (lane 8).

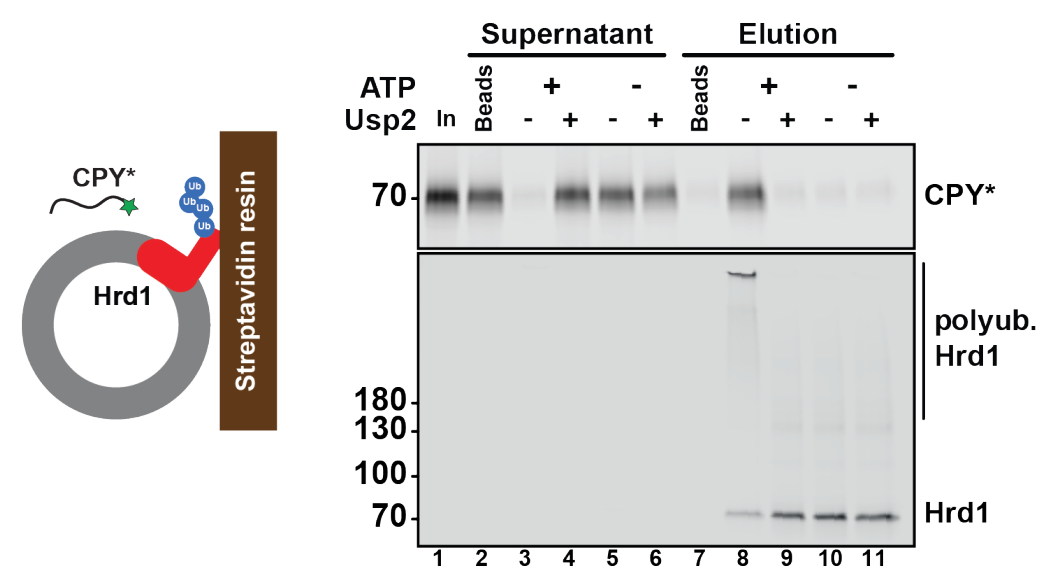

\section{Figure 3.35: Cytoplasmic region of Hrd1 binds CPY* upon autoubiquitina- tion}

Hrd1 (250 nM) reconstituted in liposomes was immobilized onto magnetic streptavidin beads and polyubiquitinated with ubiquitination machinery. Where indicated, ATP was omitted. The beads were washed and the DUB Usp2 was added where indicated. $\mathrm{CPY}^{*}(50 \mathrm{nM})$ was then added and input, unbound (supernatant) and biotin elutions were analyzed by SDS-PAGE and fluorescence scanning.

In order to determine the affinity of ubiquitinated Hrd1 for cytosolic CPY* and PrA*, I performed titration experiments. Increasing concentrations of liposome-reconstituted Hrd1 were immobilized on streptavidin magnetic beads, ubiquitinated, and incubated with $\mathrm{CPY}^{*}, \mathrm{PrA}^{*}$, or their wild-type counterparts. Cytosolic CPY* bound to ubiquitinated Hrd1 with a high affinity, whereas no binding of CPY* was observed when ATP was omitted (Figure 3.36A). Assuming a 1:1 binding stoichiometry, the apparent $K_{D}$ of ubiquitinated Hrd1 for cytosolic CPY* was approximately $7 \mathrm{nM}$. CPY WT bound less efficiently to ubiquitinated Hrd1 than CPY*, although a fraction of CPY WT did bind with similar binding kinetics as CPY*. This result explains why a fraction of CPY WT was ubiquitinated by Hrd1 (Figure 3.34A, C). No binding of CPY WT was observed when ATP was omitted from the ubiquitination reaction. Similar results were observed for $\operatorname{PrA}^{*}$, with ubiquitinated Hrd1 displaying a $K_{D}$ of approximately $120 \mathrm{nM}$ for PrA* (Figure 3.36B). No binding of PrA WT to ubiquitinated Hrd1 was observed, which also explains the low ubiquitination efficiency observed (Figure 3.34B, C). Like CPY*, no binding of PrA* or PrA WT was observed when ATP was omitted. Therefore, autoubiquitination of Hrd1 creates a high affinity binding site for misfolded 
proteins on its cytosolic side.

A

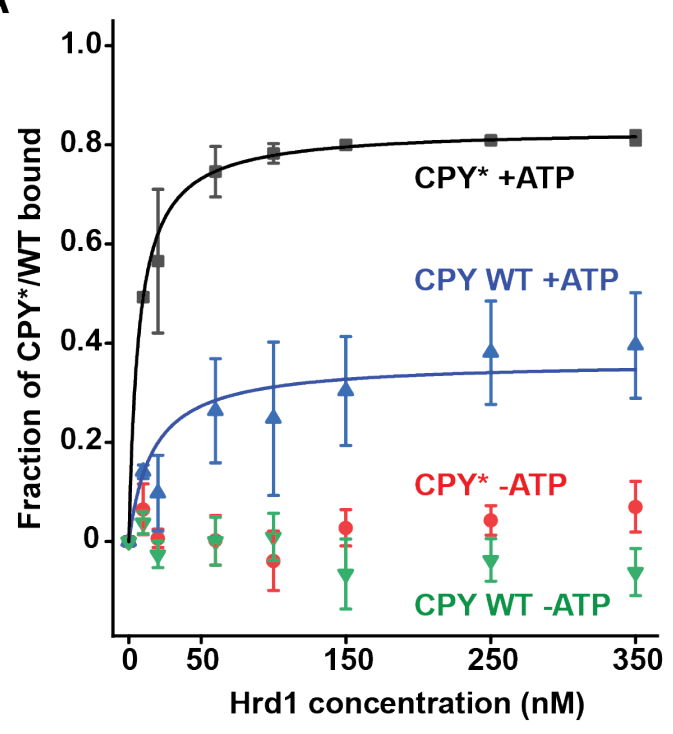

B

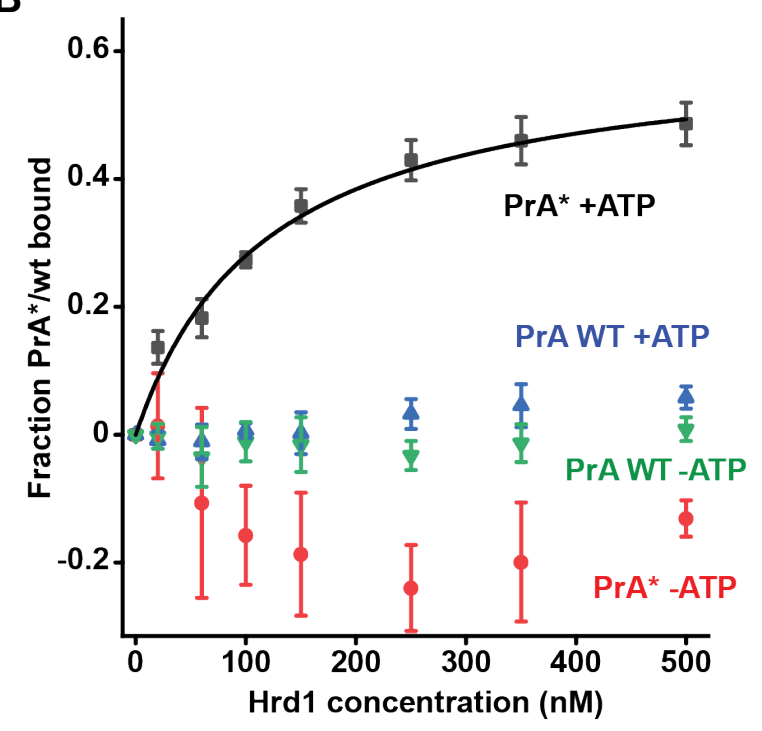

Figure 3.36: Titration of ubiquitinated Hrd1 in liposomes against cytosolic $\mathrm{CPY}^{*}, \mathrm{PrA}^{*}$

A: Increasing concentrations of Hrd1 reconstituted in liposomes were immobilized on magnetic streptavidin beads and ubiquitinated. Beads were washed and $\mathrm{CPY}^{*}$ or CPY WT $(20 \mathrm{nM})$ were added and incubated for 30 minutes. Input and unbound samples were analyzed by fluorescence scanning in a 384 well plate. Fraction bound was quantified from the unbound fraction, normalizing to the unbound fraction of the beads only control. Shown are means \pm standard deviations $(\mathrm{n}=4)$. To determine $K_{D}$, $\mathrm{CPY}^{*}$ binding was fit with a single-exponential one-site binding model. B: Titration of ubiquitinated Hrd1 against PrA* and PrA WT, as in (A). Shown are means \pm standard deviations $(\mathrm{n}=3)$.

\subsubsection{Hrd1 monoubiquitination is not sufficient for cytosolic substrate binding}

Having observed that Hrd1 autoubiquitination leads to the creation of a cytosolic binding site for misfolded proteins, I asked whether polyubiquitination of Hrd1 is required for this conformational change, or whether shorter ubiquitin chains would suffice. In other words, is there a minimal ubiquitin chain length required for creation of the binding site? To determine this, I originally planned to ubiquitinate Hrd1 with K48-linked ubiquitin chains of predetermined lengths, as was performed in a previous study with Hrd1 in detergent (Baldridge and Rapoport, 2016). However, the loading of di, tri and tetra-ubiquitin to the E1 enzyme Uba1 was inefficient, even at high concentrations of Uba1 (data not shown). Therefore, I first tested whether monoubiquitination of Hrd1 
with K48R ubiquitin, which cannot form K48-linked chains, is sufficient for cytosolic substrate binding. When reconstituted into liposomes and incubated with ubiquitination mix containing K48R ubiquitin, Hrd1 was monoubiquitinated primarily at one or two positions, and a fraction was monoubiquitinated at multiple positions (Figure 3.37A, note the single modified band vs the smear). Hrd1 liposomes were immobilized onto streptavidin magnetic beads and monoubiquitinated with K48R ubiquitin. $\mathrm{CPY}^{*}$ did not bind to monoubiquitinated Hrd1 above background binding to the beads, whereas binding was efficient when WT ubiquitin was present in the ubiquitination mix (Figure 3.37B, lanes 5 vs 3, lanes 10 vs 8). Thus, monoubiquitination of Hrd1 is not sufficient to create the cytosolic substrate binding site.

Monoubiquitinated Hrd1 was also tested in the PLB assay. We did not observe any fusions with the PLB when Hrd1 was ubiquitinated with K48R ubiquitin, indicating that monoubiquitination of Hrd1 is also not sufficient for channel formation (Figure $3.44 \mathrm{~A})$.

A

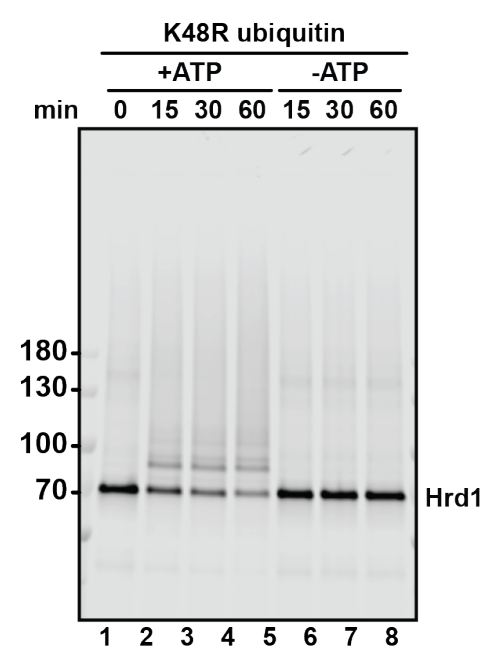

B

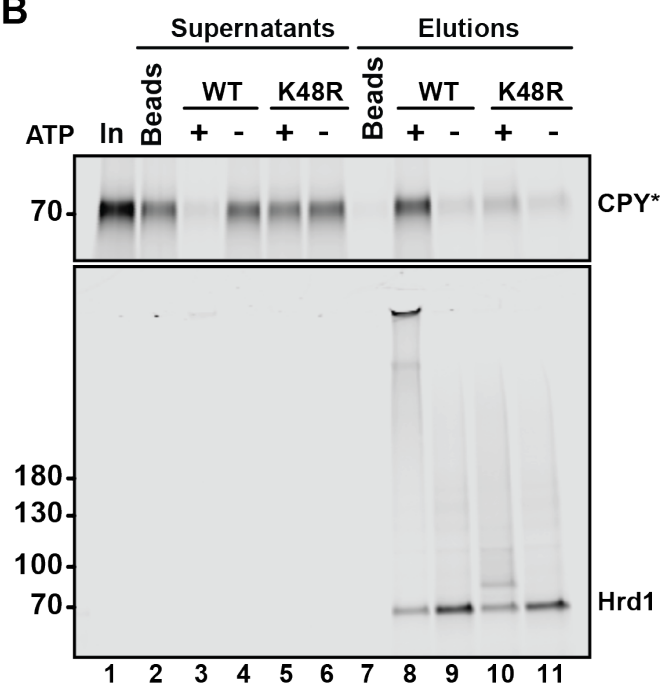

Figure 3.37: Monoubiquitinated Hrd1 does not bind cytosolic CPY*

A: Liposomes containing $\operatorname{Hrd} 1(200 \mathrm{nM})$ were incubated with ubiquitination machinery including K48R ubiquitin. Timepoints were analyzed by SDS-PAGE and fluorescence scanning. B: Hrd1 liposomes (250 nM Hrd1) were immobilized and ubiquitinated as in Figure 3.35, but with K48R ubiquitin. CPY* (40 nM) was added and input, unbound (supernatants) and biotin elutions were analyzed by SDS-PAGE and fluorescence scanning.

As another method to test $\mathrm{CPY}^{*}$ binding to multi-monoubiquitinated Hrd1, I reconstituted Hrd1 into liposomes along with the E2 enzyme Ubc6, which catalyzes multimonoubiquitination of Hrd1 in the absence of Ubc7 and Cue1-c (Figure 3.14, Weber 
et al., 2016). Hrd1/Ubc6 liposomes were immobilized onto streptavidin magnetic beads and incubated with ubiquitination machinery with or without Ubc7 and Cue1-c. Very minimal binding of $\mathrm{CPY}^{*}$ was observed when Ubc7 and Cue1-c were omitted (Figure 3.38A, lanes 6 and 15). In contrast, CPY* bound efficiently when Ubc7 and Cue1-c were included in the ubiquitination mix (Figure 3.38A, lanes 3-5, 12-14). No CPY* binding was observed in any condition when ATP was omitted, as expected (Figure 3.38A, lanes 7-10, 16-19). Ubiquitin chains attached to Hrd1 were considerably shorter in the presence of Ubc6 alone compared to when Ubc7 and Cue1-c were present (Figure $3.38 \mathrm{~B}$, lane 5 compared to lanes 2-4). Interestingly, Hrd1 was more efficiently ubiquitinated by Ubc6 and Ubc7/Cue1-c together than with Ubc7 and Cue1-c alone (Figure 3.38B, lane 4 vs 2 and 3). However, no additional CPY* binding was observed compared to when Hrd1 was ubiquitinated with only Ubc7 and Cue1-c (Figure 3.38A, lanes 5 vs 3 and 4 ).

This result confirmed that multi-monoubiquitination of Hrd1 is not sufficient for cytosolic CPY* binding. Polyubiquitination by Ubc7 and Cue1-c is clearly sufficient, but the minimum chain size required for the creation of the binding site is still unknown. 
A

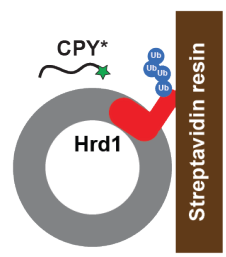

OR

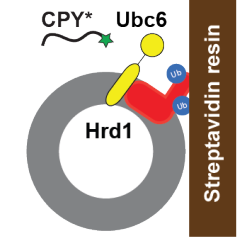

Supernatants
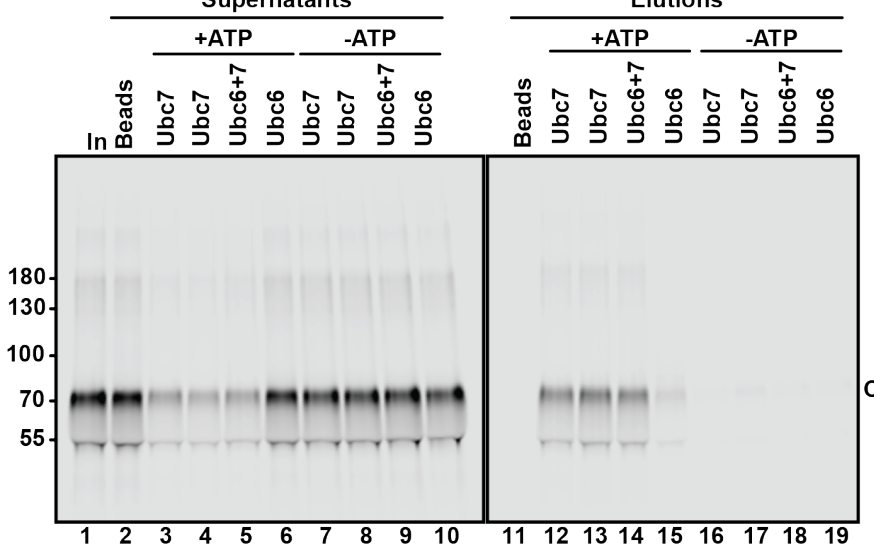

B

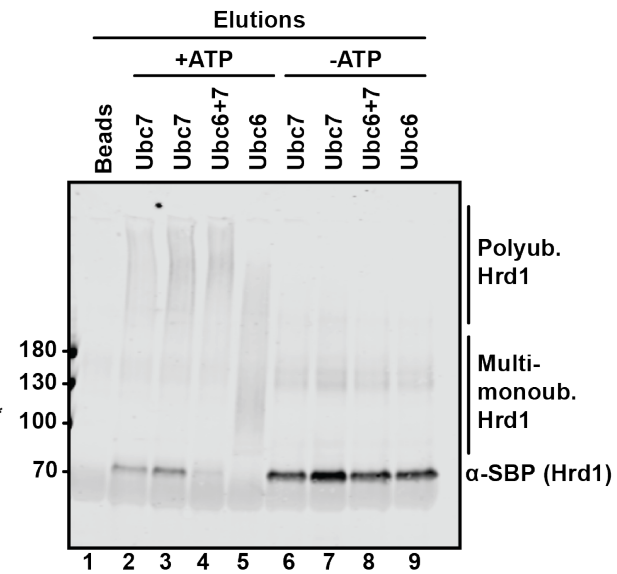

Figure 3.38: Hrd1 multi-monoubiquitination by Ubc6 does not a create cytosolic binding site

A: Hrd1 was reconstituted into liposomes alone or along with Ubc6. Liposomes (250 nM Hrd1 and Ubc6, respectively) were immobilized onto streptavidin magnetic beads and ubiquitinated with or without Ubc7 and Cue1-c. CPY* (500 nM) was then added and input, unbound (supernatants) and biotin elutions were analyzed by SDS-PAGE and fluorescence scanning to detect CPY* Lanes labeled Ubc7: Hrd1-only liposomes ubiquitinated with Ubc7 and Cue1-c. Lanes labeled Ubc6: Hrd1/Ubc6 liposomes ubiquitinated without Ubc7 and Cue1-c. Lanes labeled Ubc6+7: Hrd1/Ubc6 liposomes ubiquitinated with Ubc7 and Cue1-c. Two replicates of Ubc7 are shown. B: Biotin elutions from (A) were analyzed by western blotting against the SBP tag to detect Hrd1.

\subsection{4 $\mathrm{CPY}^{*}$ does not bind to polyubiquitin chains alone}

I next tested whether polyubiquitin chains alone, without the context of being attached to Hrd1, were sufficient to bind CPY*. To this end, I reconstituted Ubc6 containing a C-terminal SBP tag into liposomes and immobilized the liposomes onto streptavidin magnetic beads. In order to attach K48-linked polyubiquitin chains onto Ubc6, the liposomes were incubated on beads with the soluble RING domain of Doa10 along with Ubc7 and Cue1-c. As a positive control, liposomes containing Hrd1 or Hrd1 and Ubc6 were included. CPY* did not bind efficiently to polyubiquitinated Ubc6 but bound efficiently to Hrd1 and Hrd1/Ubc6 liposomes, as expected (Figure 3.39, lane 5 compared to lanes 3 and 4 and lane 12 compared to lanes 10 and 11). The 
polyubiquitin chains attached to Ubc6 appeared to be shorter than those attached to Hrd1 (Figure 3.39, lane 12 compared to 10), although this could also be explained by similar-sized ubiquitin chains attached to fewer lysine residues in Ubc6 compared to Hrd1. Therefore, I concluded that CPY* does not bind to K48-linked polyubiquitin chains alone. Rather, polyubiquitination of Hrd1 causes a conformational change which may expose a hydrophobic binding pocket for misfolded proteins.

Sup. +ATP Sup. -ATP

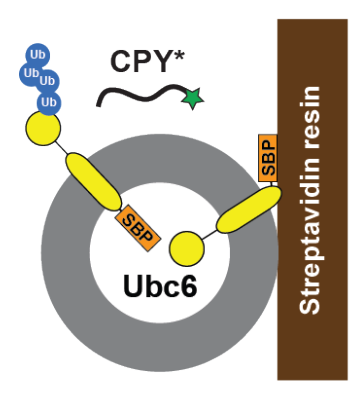

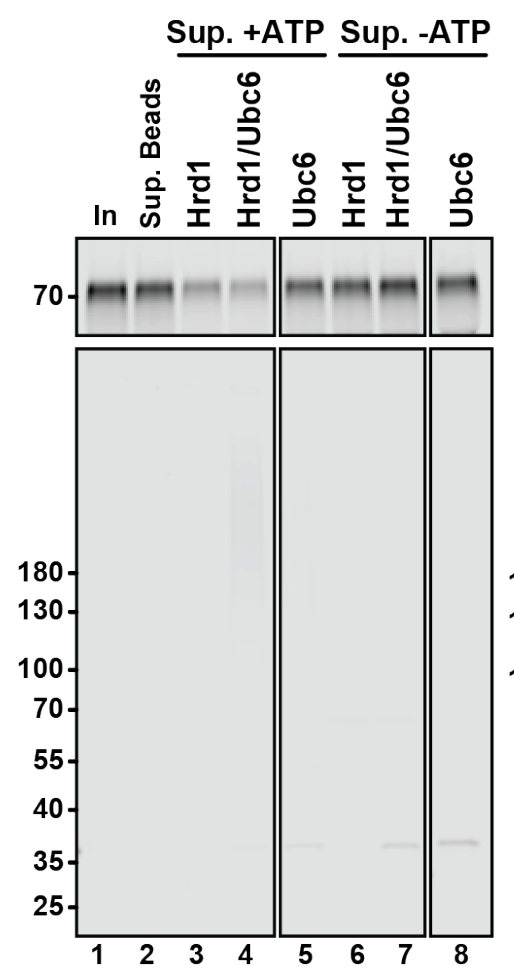

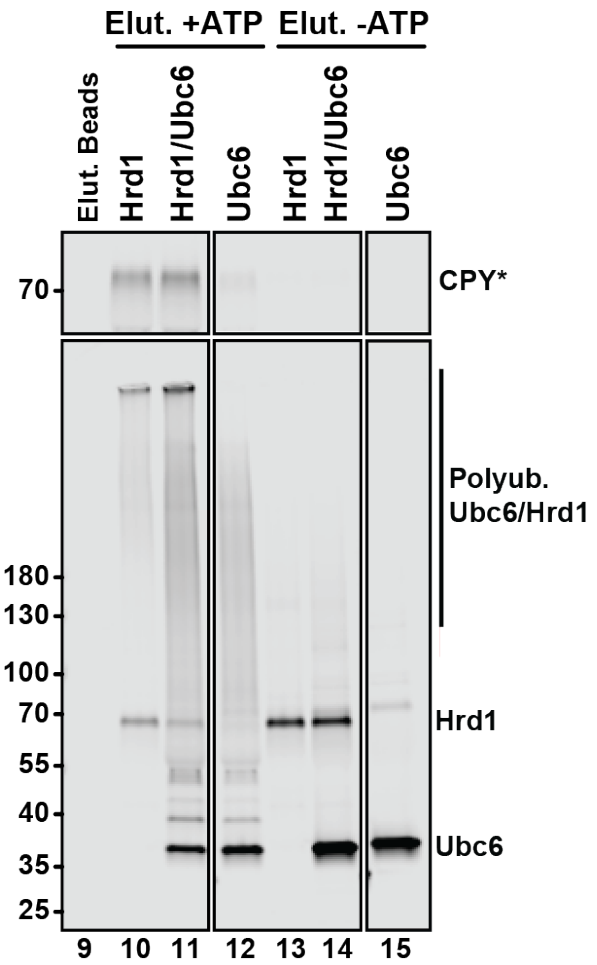

Figure 3.39: CPY* does not bind to polyubiquitin chains attached to Ubc6 Liposomes containing Ubc6-SBP (250 nM), Hrd1-SBP (250 nM), or Hrd1-SBP/Ubc6 (250 nM each) were immobilized onto streptavidin magnetic beads. Ubc6-SBP liposomes were polyubiquitinated with the Doa10 RING domain in conjunction with Ubc7 and Cue1-c. Hrd1 and Hrd1/Ubc6 liposomes were ubiquitinated with the standard ubiquitination mix containing Ubc7 and Cue1-c. CPY* (500 nM) was added and input, unbound (Sup.) and biotin elutions (Elut.) were analyzed by SDS-PAGE and fluorescence scanning. CPY* was labeled with DL800 and Hrd1 and Ubc6 were both labeled with DL680. The gel was cropped to remove irrelevant lanes.

\subsubsection{Deubiquitination of Hrd1 partially releases bound substrate}

In addition to deubiquitination closing the Hrd1 channel, I asked if deubiquitination of Hrd1 releases substrate bound on the cytoplasmic side. This release of substrate may be an important step in the retrotranslocation of substrates which do not get ubiqui- 
tinated, such as cholera toxin and yeast pro- $\alpha$-factor (Bernardi et al., 2010, 2013). In fact, the overexpression of a catalytically inactive DUB impeded cholera toxin retrotranslocation (Bernardi et al., 2013). To test if the bound substrate is released upon deubiquitination, I immobilized Hrd1 liposomes, incubated them with ubiquitination machinery, and added CPY*. The immobilized complex was treated with the DUB Usp2 and the supernatant and elution fractions were analyzed. CPY* was released into the supernatant when Usp2 was added (Figure 3.40A, lane 4 vs 3), indicating that cytosolic substrate binding was indeed reversible. Analysis of the elution fractions confirmed that Hrd1 was completely deubiquitinated after Usp2 incubation (lane 6 vs 5). About $30 \%$ of bound CPY* was released after Usp2 treatment, although Hrd1 was completely deubiquitinated (Figure 3.40B). This indicates that only a fraction of bound $\mathrm{CPY}^{*}$ was releasable, which could have been due to aggregation or $\mathrm{CPY}^{*}$ sticking to the beads after release from Hrd1.

A

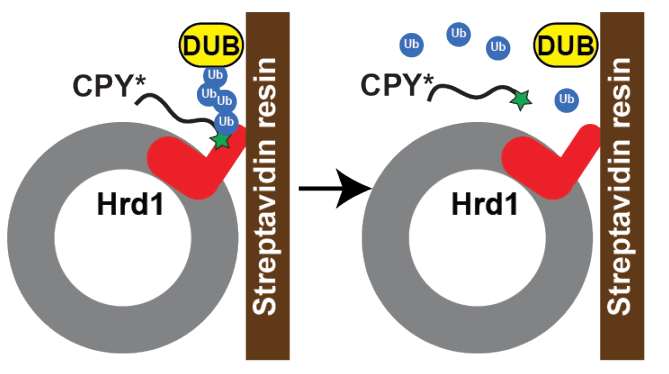

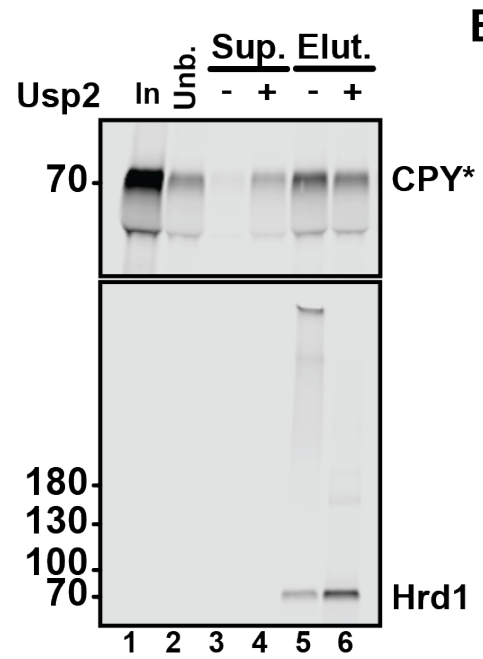

B

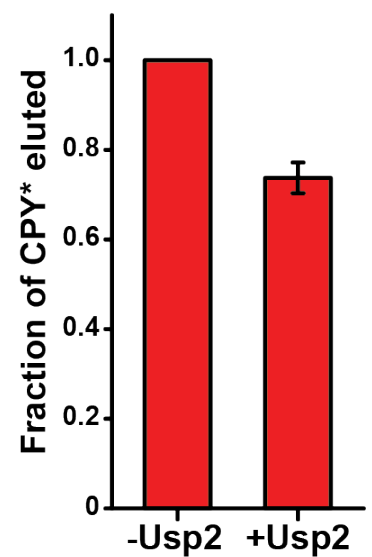

Figure 3.40: CPY* $^{*}$ partially dissociates from Hrd1 upon deubiquitination of Hrd1

A: Hrd1 $(250 \mathrm{nM})$ in liposomes was immobilized onto streptavidin magnetic resin and incubated with ubiquitination machinery. CPY* $(50 \mathrm{nM})$ was added to form a Hrd1/CPY* complex. The input and unbound (lanes 1 and 2 ) shows that the majority of $\mathrm{CPY}^{*}$ bound to Hrd1. Beads were then treated with buffer or Usp2 $(2 \mu \mathrm{M})$ and supernatants were collected. The beads were subsequently washed and eluted with biotin. Samples were analyzed by SDS-PAGE and fluorescence scanning. B: Fraction of $\mathrm{CPY}^{*}$ released was determined by quantification of the elution fractions, normalized to the sample without Usp2. Shown is mean \pm standard deviation $(n=3)$. 


\subsection{Autoubiquitination of the Hrd1 RING domain is essential for substrate binding and channel stability}

In order to determine which ubiquitination sites in Hrd1 are important for cytosolic substrate binding, I utilized mutants in which lysine residues in different regions of Hrd1 were mutated to arginine (KxR mutants, Figure 3.41A) (Stein et al., 2014). Arginine residues have a similar structure to lysine residues but cannot be ubiquitinated because they lack the primary $\varepsilon$-amine (Pickart, 2001). In detergent, some KxR mutants showed reduced autoubiquitination, but this did not completely correlate with $\mathrm{CPY}^{*}$ ubiquitination (Stein et al., 2014). This may have been due to CPY* binding to transmembrane domains that would otherwise be occluded in the membrane. The Baldridge study later demonstrated that the KRK mutant was incapable of catalyzing substrate retrotranslocation in vitro and was defective in $\mathrm{CPY}^{*}$ degradation in vivo, whereas the RKK and KKR mutants were unaffected (Baldridge and Rapoport, 2016). This study also demonstrated that three lysine residues in the RING domain of Hrd1 (373, 387 and 407, referred to as the $3 \mathrm{~K}$ mutant) were essential for CPY* degradation. With this in mind, I asked how these KxR mutants, particularly KRK, behaved in cytosolic substrate binding assays.

Because I observed that autoubiquitination of Hrd1 was critical for cytosolic substrate binding, and because autoubiquitination of the KxR mutants has never been tested in a membrane environment, I first reconstituted the KxR mutants in liposomes and checked their autoubiquitination. The KxR mutants showed significantly reduced ubiquitination efficiency compared to WT Hrd1, with KRK and 3K mutants showing slightly lower ubiquitination efficiency than KKR (Figure 3.41B and quantification in Figure 3.42A). Ubiquitination was almost completely abolished in the KRR mutant, which has no lysine residues in the cytoplasmic region. Although the KKR mutant showed a similar autoubiquitination efficiency to KRK and 3K mutants, the ubiquitin chains appeared to be longer. The results show that the lysine residues in the RING domain are the primary ubiquitination sites in Hrd1, particularly for long ubiquitin chains. The C-terminal extension is also ubiquitinated, but with shorter chains. The $3 \mathrm{~K}$ mutant showed very similar autoubiquitination efficiency to the KRK mutant, indicating that lysine residues 373,387 and 407 are the primary ubiquitination sites within the RING domain of Hrd1 (Figures 3.41B, 3.42A).

$\mathrm{CPY}^{*}$ was also included in the reaction to check the effects of differential Hrd1 autoubiq- 
uitination on substrate ubiquitination. The ubiquitination efficiency of CPY* mostly mirrored the trend in Hrd1 autoubiquitination efficiency, with KRR, KRK and 3K mutants showing markedly less CPY* ubiquitination than WT Hrd1 (Figure 3.41B and quantification in Figure 3.42B). Interestingly, The KKR mutant ubiquitinated CPY* with similar efficiency to WT Hrd1, although its overall autoubiquitination efficiency was impaired. This result indicated that autoubiquitination of the RING domain, not overall autoubiquitination efficiency, is the primary factor in efficient substrate ubiquitination. It is also worth noting that the effects on autoubiquitination and substrate ubiquitination in the KxR mutants were significantly different in the membrane environment of liposomes than what was previously observed in detergent (Stein et al., 2014).

A

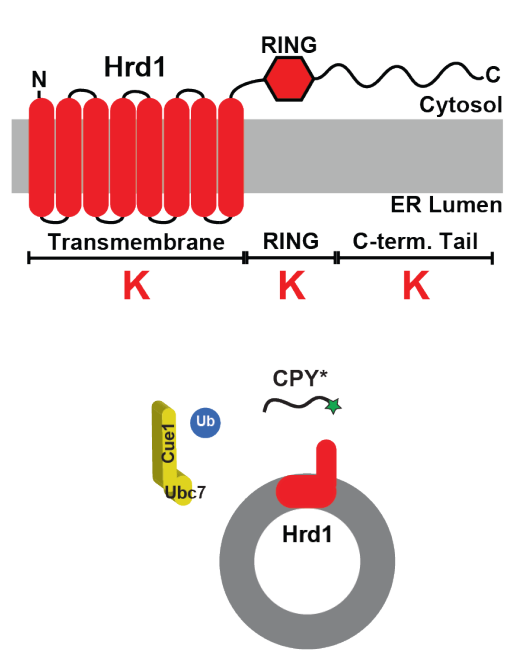

B

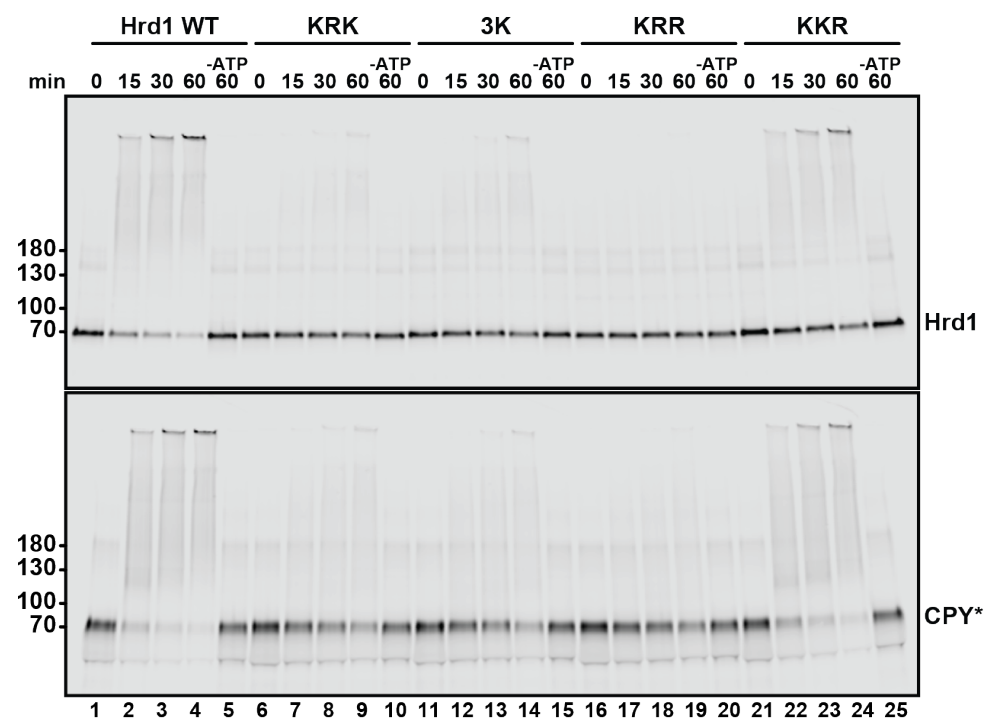

Figure 3.41: Hrd1 lysine to arginine mutants show differential autoubiquitination and $\mathrm{CPY}^{*}$ ubiquitination efficiencies

A: Top: Scheme of Hrd1 KxR mutants, in which lysine residues $(\mathrm{K})$ in different regions of Hrd1 are mutated to arginine. Below: Ubiquitination reaction scheme. $\mathrm{CPY}^{*}$ was present during ubiquitination. B: Liposomes containing Hrd1 WT or KxR mutants $(200 \mathrm{nM})$ were incubated with ubiquitination machinery in the presence of CPY* (100 $\mathrm{nM})$. Timepoints were analyzed by SDS-PAGE and fluorescence scanning. 

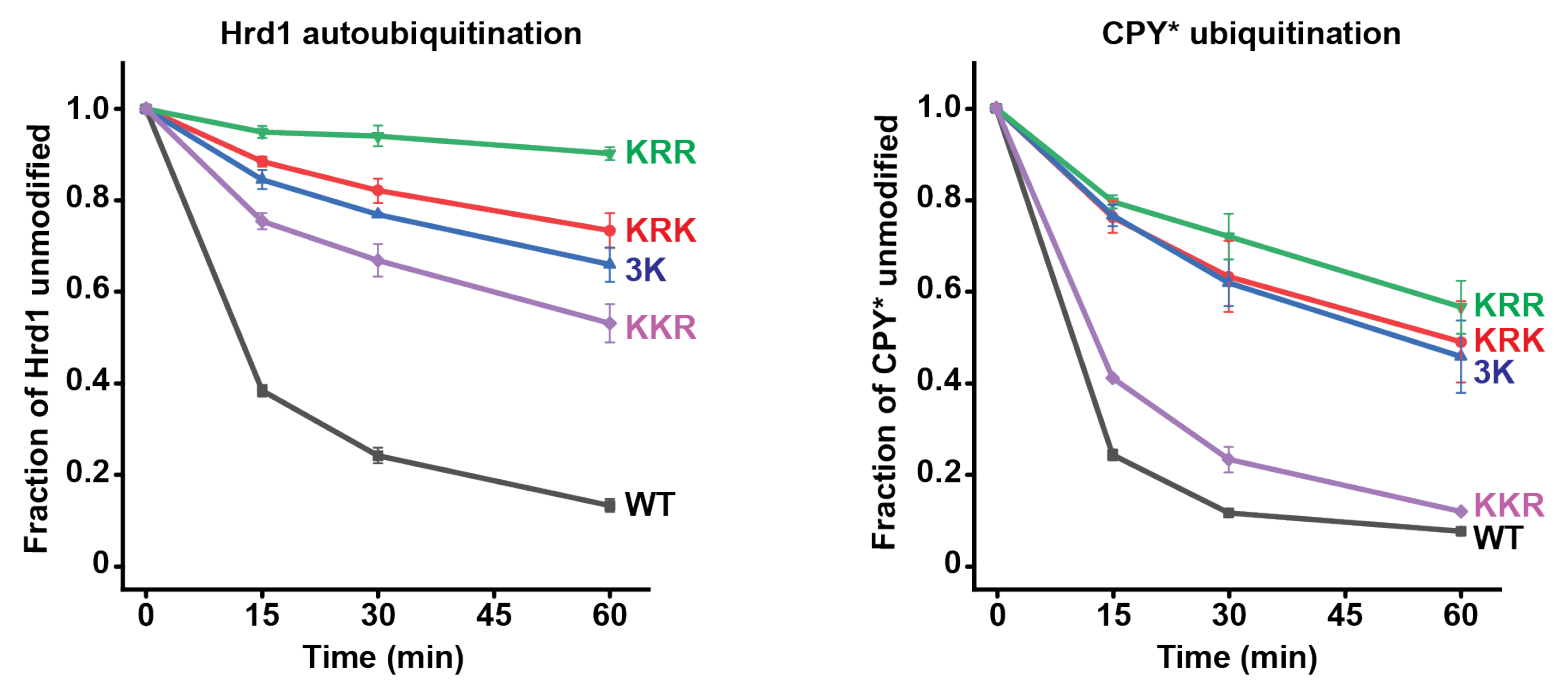

Figure 3.42: Quantification of Hrd1 autoubiquitination and CPY* ubiquitination by Hrd1 KxR mutants

Quantification of Hrd1 autoubiquitination (A) and CPY* ubiquitination (B) from Figure 3.41, normalized to the 0 min timepoint. Shown are means from three experiments. Error bars indicate standard deviation.

\subsubsection{Autoubiquitination of the Hrd1 RING domain is required for efficient cytosolic substrate binding}

I reasoned that because Hrd1 mutants lacking lysine residues in the RING domain ubiquitinated $\mathrm{CPY}^{*}$ inefficiently (Figure 3.42B), they would also have a lower affinity for cytosolic CPY*. I performed titration experiments of autoubiquitinated Hrd1 KxR mutants against $\mathrm{CPY}^{*}$ and found that indeed, the KRR, KRK and $3 \mathrm{~K}$ mutants had substantially lower affinity for CPY* than WT Hrd1 (Figure 3.43). The KRK and 3K mutants behaved identically in CPY* binding, matching what was observed for CPY* ubiquitination (compare binding in Figure 3.43 to ubiquitination in Figure 3.42B), and demonstrating that autoubiquitination in the RING domain is essential for efficient cytosolic substrate binding. The KRR mutant had a lower affinity for CPY* than $\mathrm{KRK} / 3 \mathrm{~K}$, which indicated that Hrd1 autoubiquitination at C-terminal lysine residues also has a positive effect in substrate binding. This was further supported by the observation that the KKR mutant displayed a higher affinity for $\mathrm{CPY}^{*}$ than the $\mathrm{KRK} / 3 \mathrm{~K}$ and KRR mutants, but still had a lower affinity than WT Hrd1. 

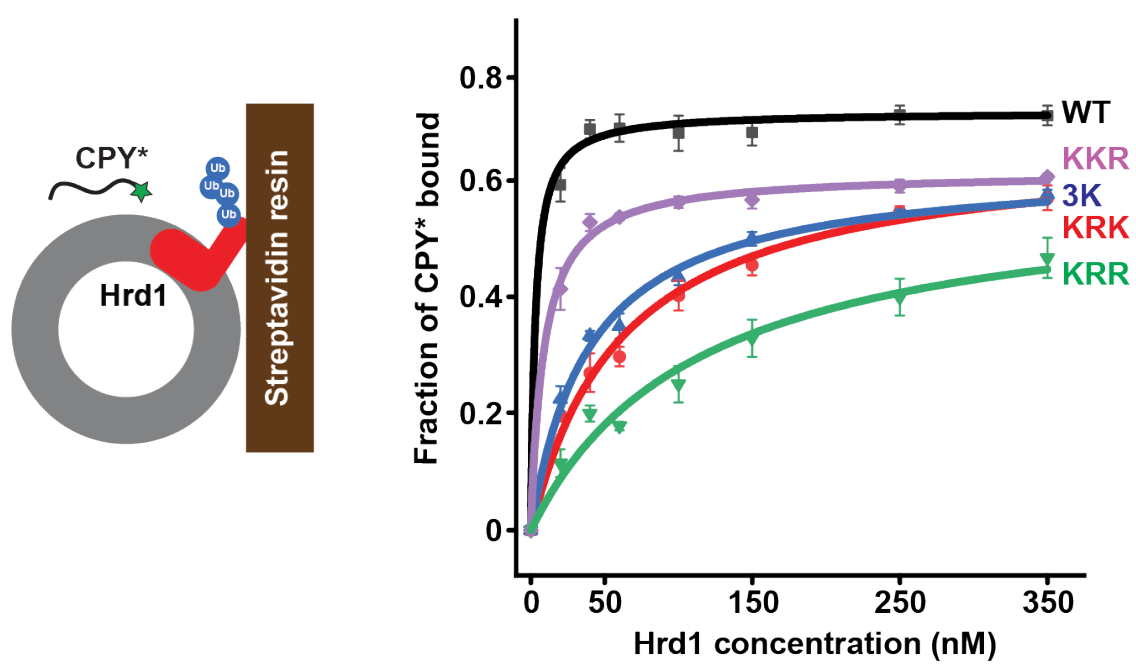

Figure 3.43: Titration of Hrd1 $\mathrm{KxR}$ mutants in liposomes against cytosolic CPY*

Liposomes containing WT Hrd1 or Hrd1 KxR mutants were immobilized on streptavidin magnetic resin at increasing concentrations, ubiquitinated, and incubated with CPY* $^{*}(20 \mathrm{nM})$. The input and unbound fractions were detected by fluorescence scanning in a 384 well plate. Fraction bound was determined by quantification of the unbound fractions, normalized to the unbound fraction in the beads only control. Shown are means \pm standard deviations $(\mathrm{n}=3)$.

Overall, these results give insight into the role of autoubiquitination of the RING domain vs cytoplasmic tail of Hrd1 and demonstrate that autoubiquitination of the RING domain is the primary factor in creation of a high affinity substrate binding site in the cytosol. This interaction on the cytoplasmic side of Hrd1 is required for efficient substrate ubiquitination.

\subsubsection{Autoubiquitination of the RING domain is essential for channel stability and substrate activation}

Autoubiquitination of the Hrd1 RING domain was shown to be essential for retrotranslocation of $\mathrm{CPY}^{*}-\mathrm{TM}$ in vitro as well as for $\mathrm{CPY}^{*}$ degradation in vivo (Baldridge and Rapoport, 2016; Neal et al., 2018). Having observed that autoubiquitination of the RING domain was critical for cytosolic substrate binding and efficient substrate polyubiquitination, we asked whether it was also important for channel activity. Using the PLB system in collaboration with Dr. Niels Denkert, we reconstituted Hrd1 KRK into liposomes, added ubiquitination machinery, and attempted to fuse these liposomes with the PLB. Interestingly, ubiquitinated KRK liposomes fused with the PLB with a 
similar rate as WT Hrd1 (Figure 3.44A), although the autoubiquitination of this mutant was significantly inhibited (Figure 3.41, 3.42). However, we observed that these channels were highly unstable and conductance was suddenly reduced to 0 within minutes (Figure 3.44B, C). The channel closure was very sudden, occurring within seconds (Figure 3.44B). The half-time of KRK channels was less than 2 minutes, whereas WT channels were stable for upwards of 30 minutes (Figure 3.44C). The channel closure appeared to be irreversible because we never observed any conductance after initial closure. When CPY* was added to both sides of the membrane after channel closure, it was unable to reactivate the closed channel (Figure 3.44D). Therefore, autoubiquitination of the RING domain is critical for channel stability. However, it is not explicitly required for channel formation, because channels did still form without RING domain autoubiquitination. It seems that residual ubiquitination in the C-terminal extension is sufficient to form short-lived channels. Accordingly, the KRR mutant, which showed almost no autoubiquitination (Figures 3.41, 3.42), was unable to fuse to the PLB, indicating that it cannot form channels (Figure 3.44A). These results provide further molecular insight into the ERAD-L defect observed in the KRK mutant (Baldridge and Rapoport, 2016; Neal et al., 2018).

We also observed that monoubiquitination of Hrd1 with K48R ubiquitin was insufficient for channel formation, as we observed no fusions to the PLB in this case (Figure 3.44A, see section 3.9.3). This mirrors the lack of cytosolic substrate binding observed with monoubiquitinated Hrd1 (Figures 3.37 and 3.38), demonstrating that channel formation and substrate binding appear to be coupled. 
A

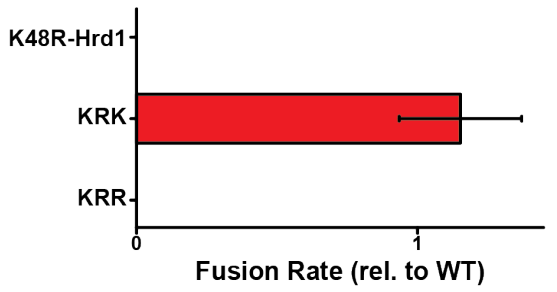

D

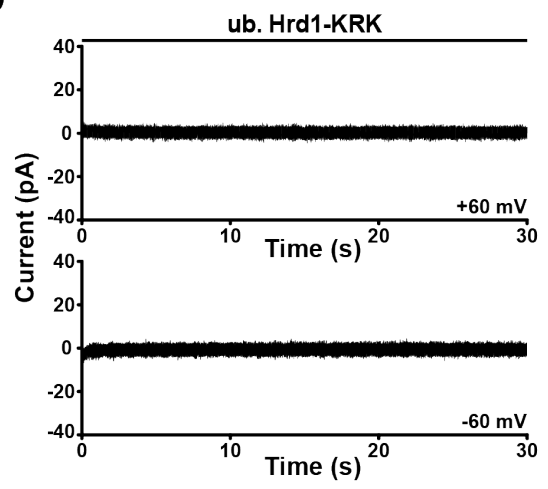

B
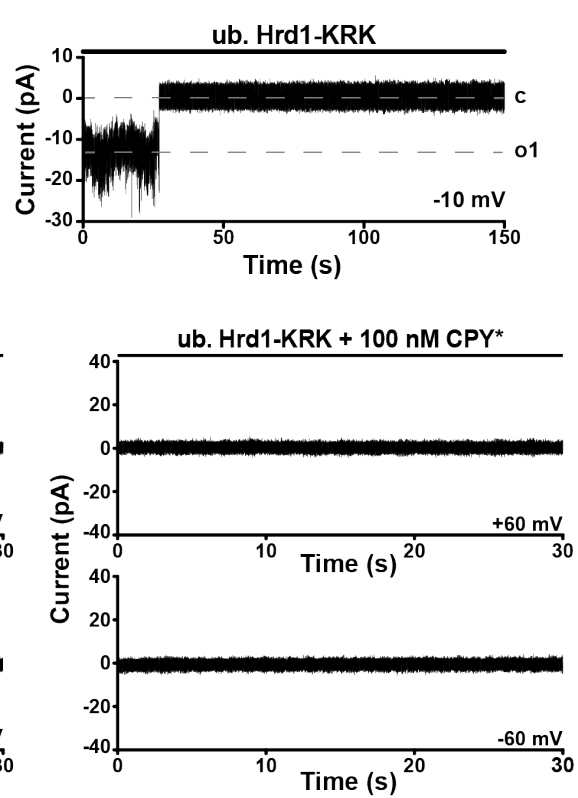

Figure 3.44: Hrd1 KRK mutant forms highly unstable channels

A: Fusion rates of polyubiquitinated KRK Hrd1, KRR Hrd1 as well as WT Hrd1 monoubiquitinated with K48R ubiquitin, (relative rate compared to polyubiquitinated WT Hrd1). Shown are means \pm SEM $(n=3)$. B: Representative current trace of a Hrd1 KRK channel, at a holding potential of $-10 \mathrm{mV}$. Notice the sudden channel closure at around $25 \mathrm{~s}$. Dashed lines refer to closed (c) and open (o1) states of the channel. C: Quantification of channel lifetime expressed as the fraction of open channels at each timepoint compared to all channels observed for WT and KRK, respectively. $n=6$ independent experiments for WT and 11 for KRK. D: Current traces of closed KRK channels at +60 and $-60 \mathrm{mV}$, without $\mathrm{CPY}^{*}$ (left) and with the addition of $100 \mathrm{nM}$ $\mathrm{CPY}^{*}$ to both sides of the chamber (right). CPY* was unable to reactivate the closed channels. Experiments and analysis were performed by Dr. Niels Denkert (University Medical Center, University of Göttingen and Max Planck Institute for Biophysical Chemistry).

\subsubsection{Lysines in the RING domain are not required for Hrd1 ubiquitin ligase activity}

I next asked whether the presence of lysines in the RING domain is required for Hrd1 ubiquitin ligase activity. It could be that mutation of lysine residues in the RING domain, such as in the KRK mutant, inhibits the activity of the RING domain. Alternatively, it might be that ubiquitin ligase activity is not affected by the presence of these lysines, and only autoubiquitination sites are affected. To differentiate between these two possibilities, I coreconstituted Hrd1 KRK with the E2 enyzme Ubc6. Be- 
cause Ubc6 has been shown to catalyze monoubiquitination of serine and threonine residues, in addition to lysine residues (Weber et al., 2016), I hypothesized that it would catalyze Hrd1 autoubiquitination even in the KRK mutant. As shown in Figure 3.45A and B, Hrd1 KRK autoubiquitination efficiency was comparable with WT Hrd1 and the KKR mutant in the presence of Ubc6 and Ubc7/Cue1-c. This indicates that Hrd1 ubiquitin ligase activity is maintained in the KRK mutant. Interestingly, cytosolic $\mathrm{CPY}^{*}$ was also ubiquitinated with similar efficiency, although the ubiquitin chains were shorter in the KRK mutant (Figure 3.45A, B). This result implies that the affinity of the KRK mutant towards cytosolic substrates was rescued by Ubc6-catalyzed autoubiquitination with Ubc7 and Cue1-c. It is not clear, however, which regions of Hrd1 are monoubiquitinated by Ubc6.
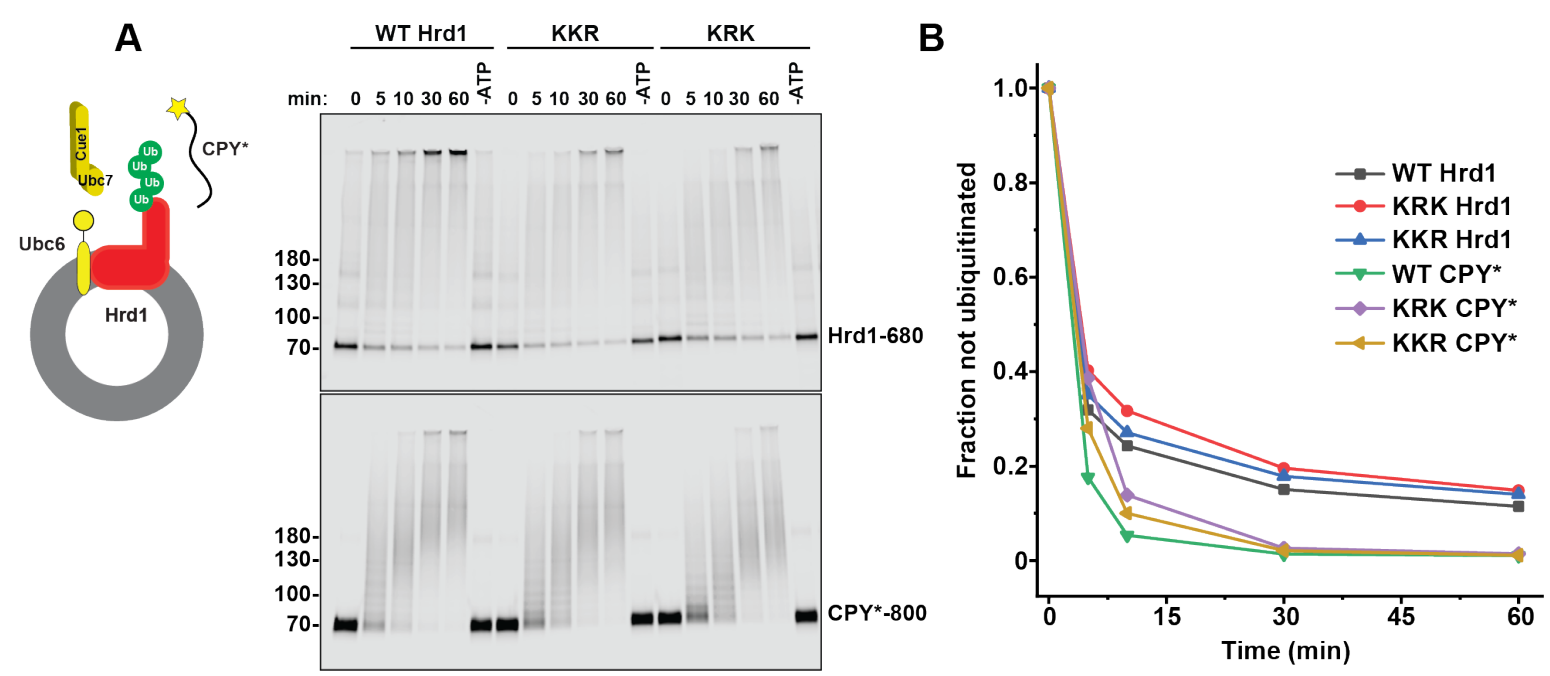

Figure 3.45: Hrd1 ubiquitin ligase activity is maintained in the KRK mutant A: Liposomes containing coreconstituted Hrd1 and Ubc6 (200 nM each) were mixed with CPY* (100 nM) and ubiquitination machinery including Ubc7/Cue1-c. Timepoints were analyzed by SDS-PAGE and fluorescence scanning. B: Quantification of Hrd1 and $\mathrm{CPY}^{*}$ ubiquitination from $(\mathrm{A})$. 


\subsection{Binding of substrates to the luminal side of Hrd1}

We observed in PLB experiments that addition of CPY* stimulated Hrd1 channel activity only when added to the luminal side (Figure 3.33). This result implied that Hrd1 contains a luminal substrate binding site. Because the luminal side of Hrd1 is not easily accessible in liposomes, I reconstituted Hrd1 into lipid nanodiscs to investigate luminal binding of substrates to Hrd1. Nanodiscs are discoidal phospholipid bilayers enclosed by amphipathic membrane scaffold proteins derived from apolipoproteins (Denisov and Sligar, 2017). Nanodiscs have the advantage that both sides of the membrane are accessible. Furthermore, they keep membrane proteins close to their native state in a phospholipid bilayer, while maintaining solubility in aqueous solutions. I reasoned that because there was no cytosolic substrate binding observed when Hrd1 was in the nonubiquitinated state, if substrate binding were to be observed to Hrd1 in nanodiscs, it would have to originate from the luminal side.

\subsubsection{Overview of Hrd1 reconstitution into nanodiscs}

Nanodiscs are formed by self-assembly of phospholipids, scaffold protein and the desired membrane proteins to be incorporated (Denisov and Sligar, 2017). Phospholipids are normally solubilized in detergent and mixed with the scaffold protein and the desired membrane protein, also in detergent. Upon gradual removal of detergent, nanodiscs self-assemble and incorporate the desired membrane protein into the phospholipid bilayer. There are a variety of factors that determine the size and shape of nanodiscs. The diameter of the nanodiscs is primarily determined by the characteristics of the scaffold protein used and the corresponding scaffold:lipid ratio. The major factors in the homogeneity and overall efficiency of membrane protein incorporation into nanodiscs are the scaffold:target protein:lipid ratio, the solubilization detergent, speed of detergent removal, lipid composition, and temperature (Denisov and Sligar, 2017).

Due to heterogeneity in the oligomeric state of Hrd1 after purification, higher molecular weight oligomers would most likely require larger nanodiscs for efficient incorporation. Of the widely-used scaffold proteins developed by the Sligar lab, the MSP2N constructs form the largest nanodiscs, with diameters of up to $17 \mathrm{~nm}$ (Grinkova et al., 2010). More recently however, studies emerged describing the use of the $22 \mathrm{kDa}$ N-terminal domain of ApoE (ApoE422K), which forms nanodiscs with diameters of up to $33 \mathrm{~nm}$ (Chromy et al., 2007; Bello et al., 2016).

For this reason, ApoE422K was chosen as the scaffold protein for reconstitution of Hrd1 
into nanodiscs. I initially used DMNG as the solubilizing detergent, because this is best compatible with Hrd1 reconstitution. To solubilize phospholipids, normally a lipid film is rehydrated in buffer containing an excess of detergent. However, DMNG was very poor in solubilizing a lipid film (personal communication from Alexander Stein). Therefore, I made empty POPC liposomes as the starting lipid material and solubilized them with an excess of DMNG (Figure 3.46). This was mixed with ApoE422K and Hrd1 in DMNG and incubated overnight. Following detergent removal, nanodiscs were characterized by size-exclusion chromatography.

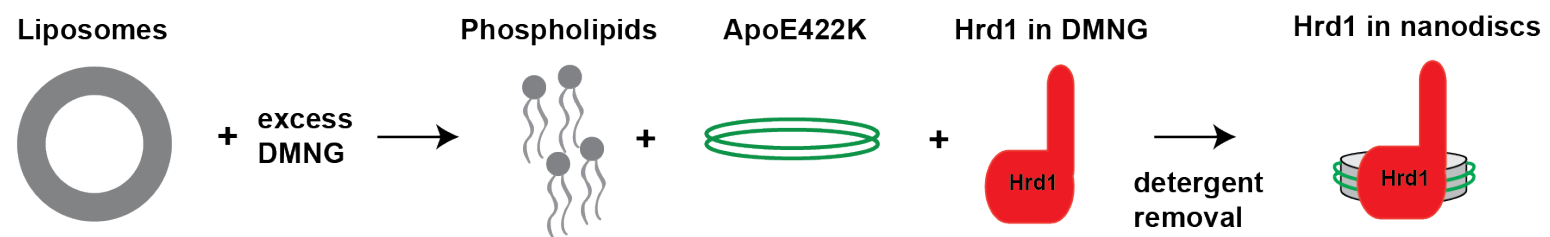

Figure 3.46: Reconstitution scheme of Hrd1 into nanodiscs by solubilization of empty liposomes with DMNG

Preformed POPC liposomes with an average diameter of $100 \mathrm{~nm}$ were solubilized with a 2.5-fold molar excess of DMNG. Following solubilization, ApoE422K and Hrd1 were added at an optimized ApoE:Hrd1:lipid ratio (see text in following section for details). Upon DMNG removal, Hrd1 nanodiscs formed by self-assembly and were characterized by size-exclusion chromatography.

\subsubsection{Optimization of nanodisc assembly with Hrd1 and ApoE using DMNG}

One of the major variables in nanodisc reconstitution is the scaffold:lipid ratio. Before incorporating Hrd1, I first screened for ratios that result in homogeneous nanodiscs. I tested ApoE:POPC molar ratios of 1:120, 1:180 and 1:290, which were previously characterized in an earlier reconstitution protocol using ApoE422K (Bello et al., 2016). Nanodisc size and homogeneity were analyzed by size exclusion chromatography on a Superose 6 5/15 GL column. Ratios of 1:180 and 1:120 produced relatively homogeneous nanodiscs, with 1:120 resulting in smaller nanodiscs than 1:180 (Figure 3.47A). At a ratio of 1:290, most of the nanodiscs eluted in the void volume, indicating that they were either very large or aggregated. Therefore, I concluded that a 1:180 ApoE:POPC ratio was a good starting point for Hrd1 reconstitution.

I next incorporated Hrd1 into nanodiscs, using an ApoE:POPC ratio of 1:160. ApoE concentration was slightly increased from 1:180 to 1:160 in order to account for the extra nanodiscs formed from the phospholipids displaced by Hrd1 incorporation. Al- 
ternatively, lipid concentration could be decreased instead of increasing scaffold concentration. I varied the ApoE:Hrd1:lipid ratio by correspondingly increasing the concentration of POPC and ApoE, which thereby decreases the Hrd1:lipid ratio. With an ApoE:Hrd1:lipid ratio of 1:0.25:160, the majority of the nanodiscs eluted in the void (Figure 3.47B, black trace). Incrementally increasing the lipid and ApoE concentration resulted in a correspondingly lower fraction of nanodiscs in the void. Overall, an ApoE:Hrd1:lipid ratio of 1:0.062:160 was determined to be optimal (Figure 3.47B, magenta trace).

A

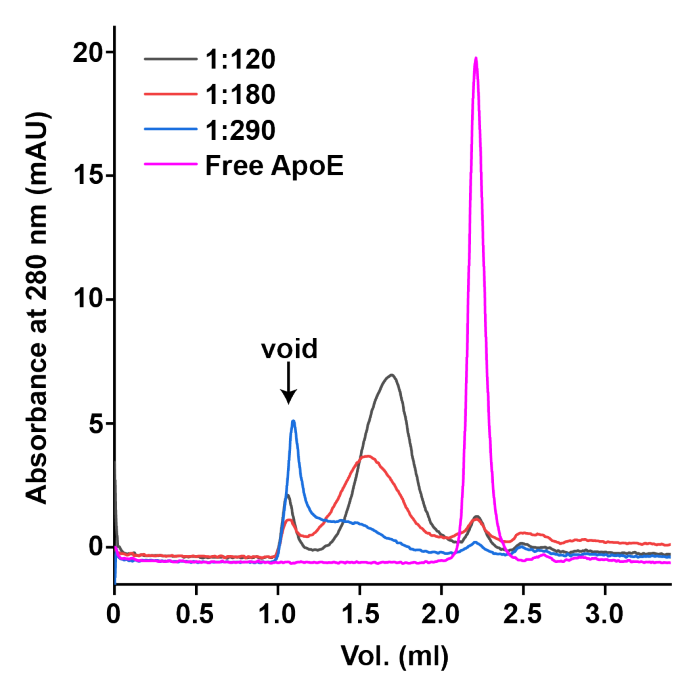

B

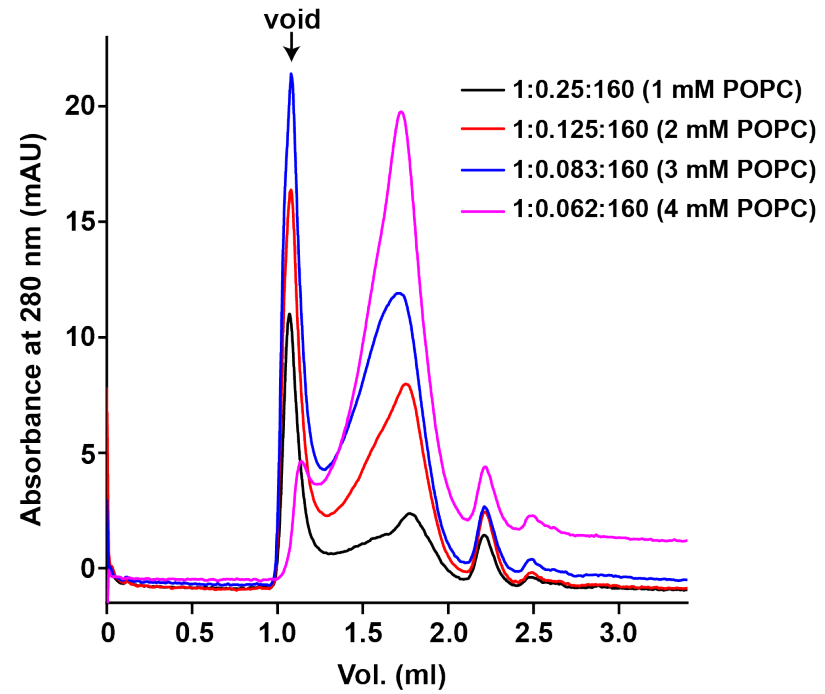

Figure 3.47: Optimization of ApoE:Hrd1:lipid ratio in nanodisc reconstitutions

A: Size exclusion chromatography of empty nanodiscs with varying ApoE:POPC ratios. Nanodiscs were injected onto a Superose 6 5/15 GL column and absorbance at $280 \mathrm{~nm}$ was monitored to detect ApoE. B: Size exclusion chromatography of Hrd1 nanodiscs with varying ApoE:Hrd1:POPC ratios on a Superose 6 5/15 GL column.

In order to further characterize the reconstitution, Hrd1 nanodiscs were prepared with the optimal 1:0.062:160 ratio and rhodamine-PE was included in the lipid mix. Nanodiscs were injected onto a Superose 6 5/15 GL column and lipids were monitored by measuring the rhodamine absorbance at $570 \mathrm{~nm}$. The lipids displayed exactly the same elution profile as the ApoE/Hrd1, measured by absorbance at $280 \mathrm{~nm}$ (Figure 3.48A). Analysis of the size-exclusion fractions by SDS-PAGE showed that the lipid-containing peak also contained Hrd1 and ApoE (Figure 3.48B). These results indicated that ApoE and Hrd1 were reconstituted into lipid-containing nanodiscs. The gel showed a higher ratio of ApoE:Hrd1 in the second half of the peak than in the first half, which likely corresponded to smaller, empty nanodiscs. This result demonstrated that Hrd1-containing 
nanodiscs could in principle be further separated from empty nanodiscs by size or density. Finally, the void also contained lipids, Hrd1, and ApoE, indicative of either very large or aggregated nanodiscs.

A

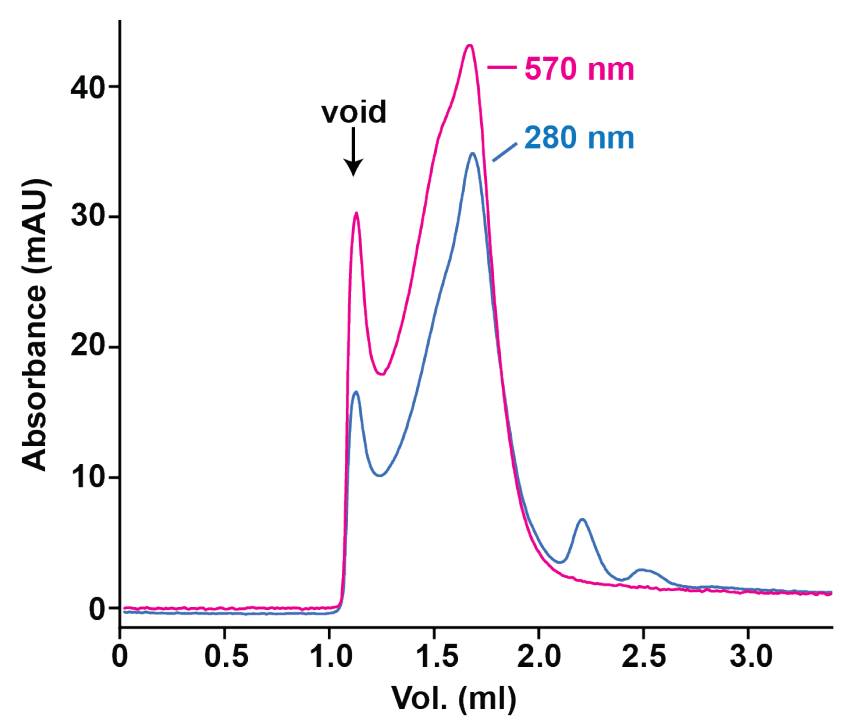

B

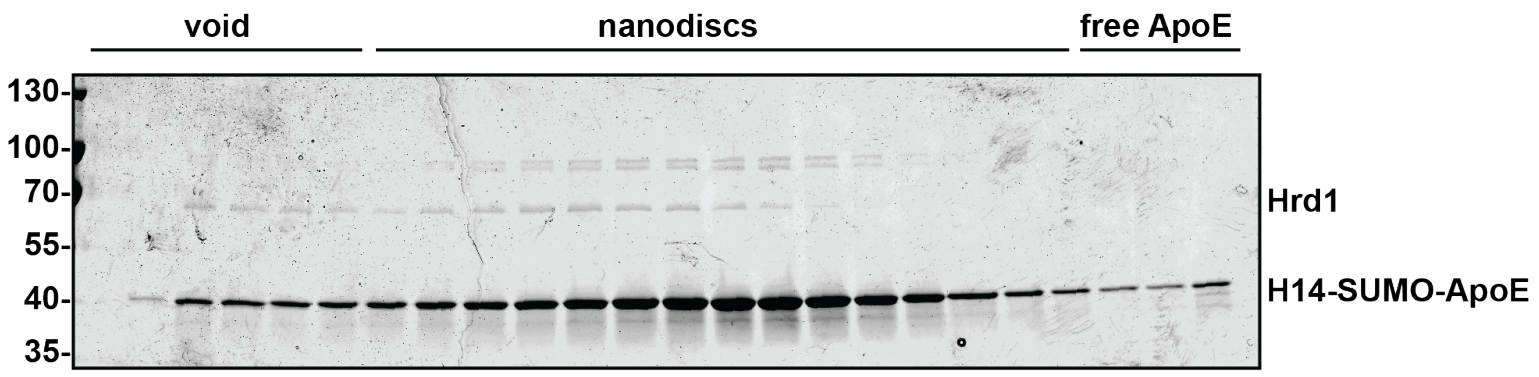

Figure 3.48: Characterization of lipid and protein content in Hrd1 nanodiscs A: Size-exclusion chromatography of Hrd1 nanodiscs with an ApoE:Hrd1:POPC ratio of 1:0.062:160, with $0.5 \%$ rhodamine-PE included in the lipid mix. Nanodiscs were injected onto a Superose 6 5/15 GL column and lipids were monitored by measuring absorbance at $570 \mathrm{~nm}$. ApoE and Hrd1 were monitored by measuring absorbance at $280 \mathrm{~nm}$. B: Fractions from size exclusion chromatography in (A) were analyzed by SDS-PAGE and coomassie staining.

\subsubsection{Reconstitution of Hrd1 into nanodiscs using DM}

Using DMNG to solubilize performed liposomes was a viable method to reconstitute Hrd1 into ApoE nanodiscs, but there was a potential for liposome contamination from incomplete solubilization of the liposomes. In the meantime, I observed that DM was a viable detergent for Hrd1 reconstitution into liposomes (Section 3.3.1). DM is also capable of fully solubilizing a lipid film at high concentrations, which avoids 
the contamination of partially-solubilized liposomes. Therefore, a POPC lipid film containing $0.5 \%$ rhodamine PE was solubilized with $2 \% \mathrm{DM}$ and was used as the lipid source, modifying the nanodisc reconstitution described in sections 3.11.1-3.11.2. After detergent removal, DM nanodiscs were analyzed by size exclusion chromatography using a Superose 6 10/300 GL column. DM-mediated reconstitution resulted in fewer nanodiscs eluting in the void than the DMNG method (Figure 3.49A). Additionally, lipids coeluted with ApoE and Hrd1, as determined by absorbance of rhodamine-PE at $560 \mathrm{~nm}$. Therefore, I proceeded from here on with DM-mediated reconstitution of Hrd1 into nanodiscs.

In order to better separate Hrd1-containing nanodiscs from empty nanodiscs, the nanodiscs were purified by glycerol gradient centrifugation. This method separates nanodiscs based on density, whereas size-exclusion chromatography separates nanodiscs based on the hydrodynamic radius. Hrd1 and ApoE were present in two overlapping peaks after gradient centrifugation, indicating that Hrd1-containing nanodiscs were separated from empty nanodiscs (Figure 3.49B). This demonstrated that Hrd1containing nanodiscs were denser than empty nanodiscs, in addition to having a larger hydrodynamic radius (Figure 3.48). The Hrd1-containing fractions were pooled and further characterized.

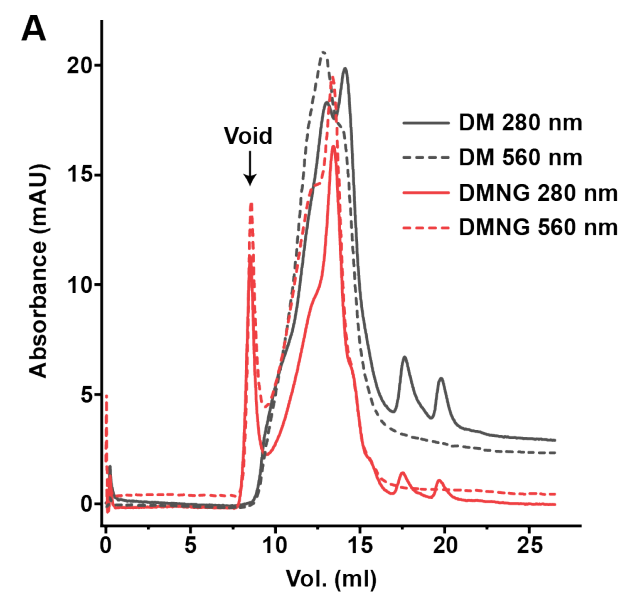

B

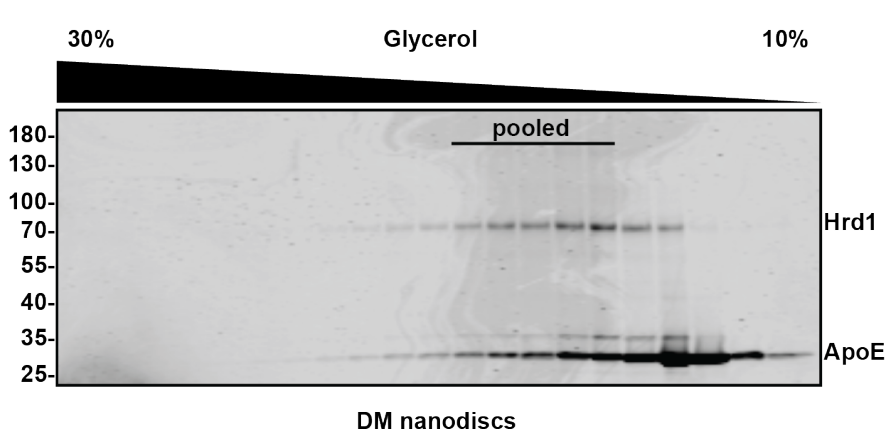

Figure 3.49: Reconstitution of Hrd1 into nanodiscs with DM

A: Hrd1 was reconstituted into nanodiscs using either DMNG-solubilized POPC liposomes (red) or a DM-solubilized POPC lipid film (black). Both lipid sources contained 0.5\% rhodamine PE. Nanodiscs were injected onto a Superose 6 10/300 GL column and protein and lipid content were monitored by absorbance at 280 and $560 \mathrm{~nm}$, respectively. B: Hrd1 was reconstituted into nanodiscs using the DM-lipid film method and the nanodiscs were centrifuged in a 10-30\% (w/v) glycerol gradient. Fractions were analyzed by SDS-PAGE and coomassie staining. 


\subsubsection{Structural and biochemical characterization of Hrd1 nanodiscs}

After purification of Hrd1 nanodiscs by glycerol gradient centrifugation, they were structurally characterized by negative stain electron microscopy (Dr. Dietmar Riedel, Department of Structural Dynamics, Max Planck Institute for Biophysical Chemistry). The nanodiscs were heterogeneous in size, primarily ranging from 20-60 nm in diameter (Figure 3.50A, B). The heterogeneity was most likely because Hrd1 is in a heterogeneous oligomeric state after purification. The electron microscopy analysis also showed that the preparation was almost entirely free of liposome contamination, because liposomes normally appear as deflated spheres in negative stain electron microscopy (Franken et al., 2017). The discoidal structures we observed were completely flat.

Gradient-purified Hrd1 nanodiscs were additionally characterized by a pull-down experiment to check for the fraction of empty nanodiscs in the sample. Nanodiscs were immobilized on streptavidin magnetic beads through the SBP tag of Hrd1. ApoE was almost completely co-depleted with Hrd1, indicating that empty nanodiscs were efficiently removed by gradient centrifugation (Figure 3.50C). 
A

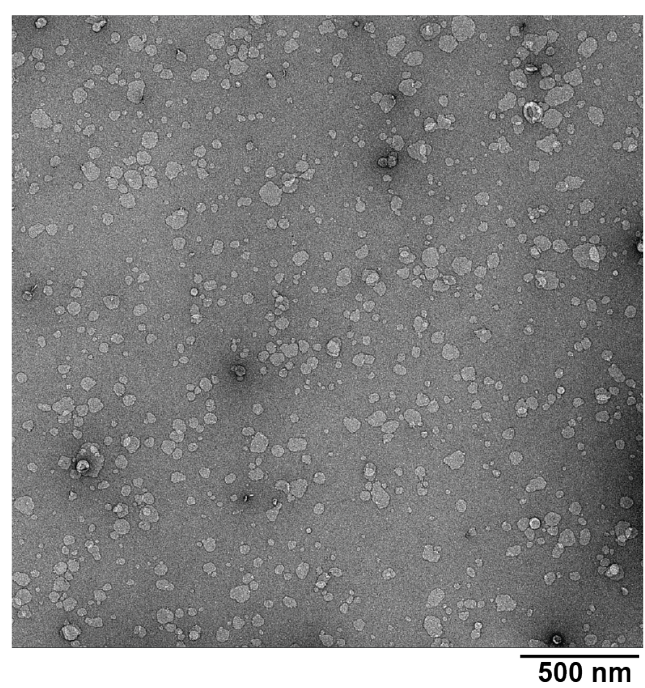

B

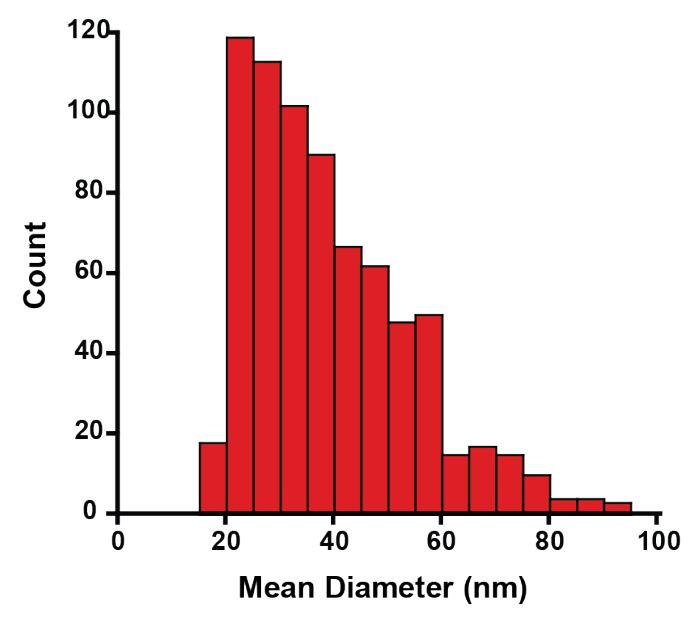

C

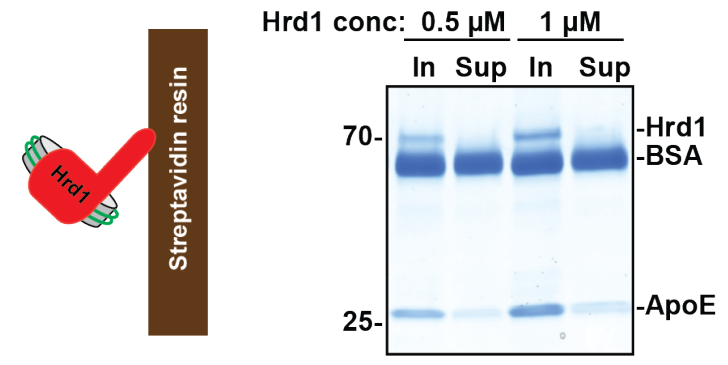

Figure 3.50: Structural and biochemical characterization of Hrd1 nanodiscs A: Representative negative-stain electron microscopy image of Hrd1 nanodiscs. B: Size distribution histogram of Hrd1 nanodiscs. Electron microscopy and size distribution analysis was performed by Dr. Dietmar Riedel, Department of Structural Dynamics, Max Planck Institute for Biophysical Chemistry. C: Hrd1 nanodiscs at two different concentrations of Hrd1 were immobilized on streptavidin magnetic beads through the SBP-tag of Hrd1. BSA was included to reduce non-specific binding to the beads. Input and unbound fractions (In and Sup) were analyzed by SDS-PAGE and coomassie staining.

\subsubsection{Hrd1 ubiquitination activity in nanodiscs}

The activity of Hrd1 reconstituted in nanodiscs was determined by a ubiquitination assay in the presence of CPY*. Hrd1 autoubiquitination in nanodiscs was comparable in both kinetics and overall efficiency to Hrd1 in liposomes (Figure 3.51A, C). Likewise, Hrd1 ubiquitinated CPY* with similar efficiency and kinetics in nanodiscs compared to Hrd1 in liposomes (Figure 3.51B, C). The presence of CPY* did not affect Hrd1 autoubiquitination kinetics compared to Hrd1 nanodiscs alone. Therefore, Hrd1 
reconstituted in ApoE nanodiscs is active.

A

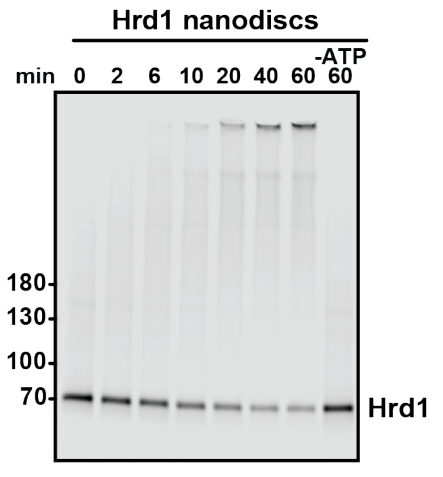

B

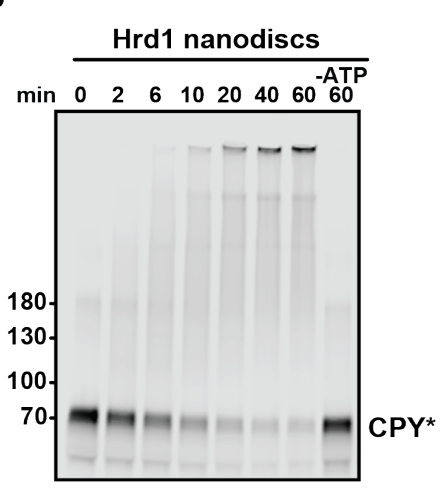

C

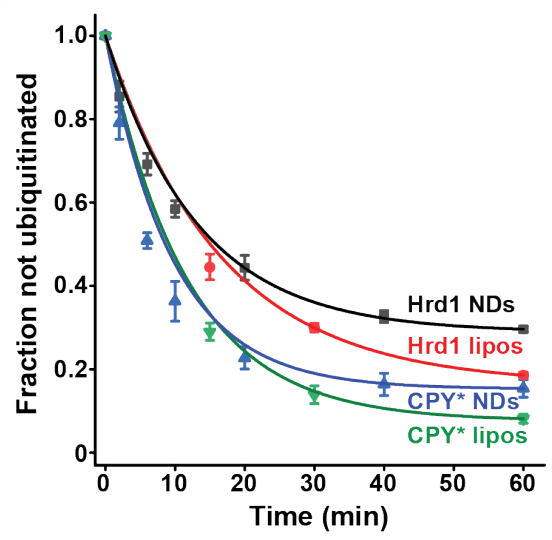

Figure 3.51: Ubiquitination activity of Hrd1 in nanodiscs

A, B: Hrd1 nanodiscs (200 nM Hrd1) were incubated with ubiquitination machinery in the presence of CPY* $(100 \mathrm{nM})$. Timepoints were analyzed by SDS-PAGE and fluorescence scanning. C: Quantification of Hrd1 autoubiquitination and CPY* ubiquitination in nanodiscs and liposomes, from three experiments. The data for the liposome experiments is the same as in Figure 3.34, plotted here for comparison purposes. Shown are means \pm standard deviations. The data was fit with a single-exponential decay function for visualization of ubiquitination kinetics.

\subsection{6 $\mathrm{CPY}^{*}$ binds to nonubiquitinated Hrd1 in nanodiscs}

Using Hrd1 reconstituted in nanodiscs, binding of $\mathrm{CPY}^{*}$ to non-ubiquitinated Hrd1 was investigated. Hrd1 nanodiscs were immobilized onto streptavidin magnetic beads and incubated with increasing concentrations of CPY* or CPY WT. Strikingly, CPY* bound to non-ubiquitinated Hrd1, and the apparent $K_{D}$ of the interaction was approximately $150 \mathrm{nM}$ (Figure 3.52). In contrast, CPY WT showed almost no binding to non-ubiquitinated Hrd1 across the range of Hrd1 concentrations tested. Considering that non-ubiquitinated Hrd1 in liposomes (where only the cytoplasmic side is accessible) showed no affinity to $\mathrm{CPY}^{*}$, the binding to Hrd1 nanodiscs most likely originated from the luminal side of Hrd1. This result demonstrates that Hrd1 alone can differentiate misfolded proteins from correctly folded proteins with its luminal domain.

When Hrd1 nanodiscs were ubiquitinated on beads, the affinity for CPY* drastically increased (Figure 3.52), mirroring what was observed with ubiquitinated Hrd1 in liposomes (Figure 3.36). CPY WT also partially bound to ubiquitinated Hrd1 in nanodiscs, similar to what was observed with ubiquitinated Hrd1 in liposomes. The binding observed to ubiquitinated Hrd1 in nanodiscs is most likely from the cytosolic binding 
site, although it cannot be excluded that binding to the luminal side also occurs in the ubiquitinated state. Because data points at very low Hrd1 concentrations were missing, it was not possible to determine a reliable $K_{D}$ value for ubiquitinated Hrd1 in nanodiscs and CPY*.

Overall, these data indicated that $\mathrm{CPY}^{*}$ most likely binds to the luminal side of Hrd1, with a $K_{D}$ one order of magnitude lower than the cytosolic binding observed with ubiquitinated Hrd1 in liposomes (approximately $150 \mathrm{nM}$ vs $7 \mathrm{nM}$, respectively. Compare Figure 3.52 with Figure 3.36). This affinity gradient for substrates from the luminal to the cytosolic side may provide the initial driving force and directionality during retrotranslocation of misfolded proteins through Hrd1 (see Discussion, section 4.6 for further details).
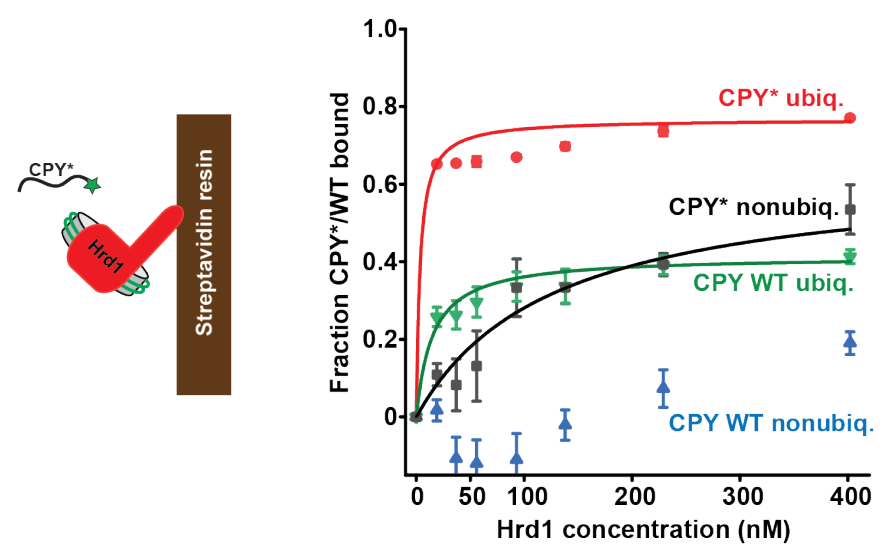

\section{Figure 3.52: Differential binding of $\mathrm{CPY}^{*}$ to nonubiquitinated and ubiqui- tinated Hrd1 in nanodiscs}

Increasing concentrations of Hrd1 were immobilized onto streptavidin magnetic beads and incubated with $20 \mathrm{nM} \mathrm{CPY*}$ or $20 \mathrm{nM}$ CPY WT (referred to as nonubiq.). Alternatively, Hrd1 nanodiscs were poly-ubiquitinated on beads with ubiquitination mix and then incubated with $20 \mathrm{nM}$ CPY* or $20 \mathrm{nM}$ CPY WT (referred to as ubiq.). The input and unbound fractions were analyzed by fluorescence scanning in a 384 well plate. Fraction bound was quantified from the unbound fraction, normalized to the unbound fraction in the beads-only control. Shown are means \pm standard deviation from three experiments. Data were fit with a single-exponential one-site binding model, from which $K_{D}$ for $\mathrm{CPY}^{*}$ binding to nonubiquitinated Hrd1 was derived. 



\section{Discussion}

\subsection{The fusion system as a method to study retrotranslocation}

This thesis concerned itself with understanding how luminal misfolded proteins get retrotranslocated by the Hrd1 complex during ERAD. Of the different classes of substrates degraded by the Hrd1 complex, luminal soluble misfolded proteins are of particular interest because they require a protein-conducting channel spanning the entire ER membrane. As it is not clear how the Hrd1 complex accomplishes this feat, I aimed to reconstitute the process with purified components to investigate the mechanism of retrotranslocation in detail. Reconstitution with purified components was the method of choice, because this allows for the sufficiency of individual components to be addressed, as well as direct mechanistic details to be drawn.

A substantial amount of evidence has led to the hypothesis that the Hrd1 ubiquitin ligase forms the retrotranslocon, while the other components in the complex perform regulatory functions (Carvalho et al., 2010; Stein et al., 2014; Baldridge and Rapoport, 2016; Schoebel et al., 2017) (see section 1.9). Two previous reconstitutions of Hrd1mediated retrotranslocation with purified components formed the basis for much of the current biochemical understanding of Hrd1. However, these studies did not faithfully recapitulate the retrotranslocation of luminal substrates due to technical reasons or experimental design (Stein et al., 2014; Baldridge and Rapoport, 2016). In the study by Stein and colleagues, a Hrd1 and CPY* complex was formed in detergent, which was coreconstituted into liposomes. Although $\mathrm{CPY}^{*}$ was ubiquitinated by Hrd1 and extracted from the membrane by the Cdc48 complex, CPY* was accessible to proteases, indicating that it was not encapsulated in the lumen (Stein et al., 2014). In the study by Baldridge and Rapoport, CPY* was encapsulated into liposomes through the use of a transmembrane domain. Thereafter, the liposomes were solubilized with detergent in order to incorporate Hrd1. Strikingly, CPY* was exposed to the cytosol upon Hrd1 autoubiquitination, showing that Hrd1 retrotranslocates CPY* (Baldridge and 
Rapoport, 2016). However, due to the possibility that the transmembrane domains of Hrd1 interacted with $\mathrm{CPY}^{*}$ during detergent-mediated Hrd1 incorporation, it is still not clear if Hrd1 can retrotranslocate a luminal substrate "de novo" (see section 1.10.1). To overcome these limitations, I encapsulated CPY* into liposomes and delivered it to Hrd1-containing liposomes by SNARE-mediated fusion (see section 3.1.1). This fusion system recapitulates retrotranslocation faithfully because it allows Hrd1 to access a substrate on its luminal side without the use of detergent. I then initiated retrotranslocation by adding components of the cytosolic ubiquitination machinery, as Hrd1 autoubiquitination was demonstrated to open the channel (Baldridge and Rapoport, 2016). I monitored the ubiquitination status of the substrate as a readout for retrotranslocation, since it can only be ubiquitinated when it emerges into the cytosol. I was not able to conclusively demonstrate with this system that Hrd1 can retrotranslocate luminal substrates. However, several technical limitations such as substrate aggregation made it difficult to draw conclusions about the role of Hrd1 in retrotranslocation. In addition, developing the system was not trivial and required significant optimization. Several of the techniques I established, including mixing membrane proteins and encapsulated proteins via SNARE-mediated fusion, are applicable in many fields of membrane protein research. I believe that the fusion system will be a useful method for studying retrotranslocation once certain technical issues are solved. The advancements I made in developing the fusion system and its limitations are described in the following sections.

\subsubsection{Soluble substrates can be efficiently encapsulated into liposomes}

One of the novelties in the fusion system is that soluble ERAD-L substrates are efficiently encapsulated into liposomes without the use of a transmembrane domain. There were several challenges to overcome to accomplish this. First, the luminal volume of the liposomes is very small. As an example, an $8 \mathrm{mM}$ solution of POPC liposomes with $100 \mathrm{~nm}$ diameter, assuming a bilayer thickness of $2.5 \mathrm{~nm}$, has only $2 \%$ of the total volume entrapped in the lumen (based on the calculation in Adamala et al., 2016). The actual entrapped volume is likely even lower because the liposomes I used contained cholesterol, which increases the thickness of the bilayer (Nezil and Bloom, 1992). Overall, this means that for efficient encapsulation, a large excess of the protein is required in the reconstitution mix, as is normally the case when encapsulating smaller compounds such as fluorescent dyes or oligonucleotides (Kyoung et al., 2011; Nickel 
et al., 1999). Alternatively, one can increase the entrapped volume by using a high lipid concentration, as was done in a previous study that successfully encapsulated GFP and streptavidin (Heo et al., 2017). There, the lipid concentration was very high, at $100 \mathrm{mM}$. Another possibility is to use liposomes with larger diameters.

The encapsulation of tightly-folded, highly-soluble proteins such as GFP is more straightforward than misfolded proteins. Misfolded proteins aggregate in a concentrationdependent manner in the absence of detergent or denaturing agent. This meant that I could not use a high starting concentration in the reconstitutions. My initial trials of using excess sCPY* during detergent solubilization resulted in no encapsulation (Figure 3.4). I therefore turned to an affinity-based approach. By using NiNTA-labeled phospholipids and a His-tagged substrate, I was able to overcome the inefficiencies of encapsulation at relatively low substrate concentrations, by tethering the substrate to the lipids during reconstitution. The NiNTA approach resulted in encapsulation efficiencies of up to $30 \%$ for $\mathrm{CPY}^{*}$ (Figure 3.6), and was compatible with $\Delta \mathrm{N}$ complex reconstitution protocols, resulting in efficient lipid mixing (Figures 3.13, 3.14) and content mixing (Figure 3.15). Non-encapsulated CPY* was efficiently removed by flotation in a Nycodenz gradient containing imidazole and urea, resulting in $85 \%$ of CPY* protected from proteinase K (Figure 3.7). This provides a good starting point for detection of retrotranslocation.

The NiNTA method was also broadly applicable for the encapsulation of other substrates and enzymes. His-tagged PrA*, sCPY*-GFP, and the SUMO protease Ulp1 were efficiently encapsulated (Figures 3.23, 3.21, 3.15). Ulp1 was active after encapsulation (Figure 3.15), showing that the NiNTA encapsulation protocol is compatible with experiments where enzymatic activity needs to be maintained.

\subsubsection{SNARE-mediated fusion delivers substrates to the luminal side of Hrd1}

I characterized both lipid mixing and content mixing between Hrd1 and substrate liposomes to exclude the possibility that fusion was the bottleneck in the system. Overall, the fusion system effectively mixes both membrane and luminal compartments. Standard fluorescence dequenching assays showed efficient membrane mixing (Figure 3.13). Additionally, I developed two methods that allow for biochemical detection of membrane and content mixing. This was important because bulk assays involving fluorescence dequenching and FRET do not give information about subpopulations of liposomes or the activity of the proteins within them. 


\subsubsection{Ubc6 autoubiquitination assay demonstrates mixing of membrane components}

The membrane mixing assay using Ubc6 and Hrd1 in separate liposomes showed that Hrd1 efficiently autoubiquitinated in a SNARE-dependent manner (Figure 3.14). Autoubiquitination was strongly reduced when fusion was inhibited with $\mathrm{Syb}_{\text {sol }}$, showing that SNARE complex formation and membrane mixing were required for efficient Hrd1 autoubiquitination. There was some residual autoubiquitination in the inhibited fusion reaction, showing that Ubc6 has some E2 activity in trans. This assay does not exclude that tight docking of liposomes increases the trans E2 activity of membraneanchored Ubc6. However, taken together with the lipid mixing observed in fluorescence dequenching assays as well as the substrate cleavage in luminal content mixing assays, this was likely not the case.

Other biochemical assays for membrane mixing are present in the literature. One study demonstrated the reconstitution of reactions in the bacterial respiratory chain using SNARE-mediated fusion of liposomes (Nordlund et al., 2014). This study monitored ATP synthesis as a readout for fusion. Although useful, monitoring the appearance of ATP over time, without a positive control, does not report on the overall efficiency of fusion. The Ubc6 autoubiquitination assay shows both the appearance of a signal (ubiquitinated Hrd1) and the disappearance of the starting product (unmodified Hrd1), allowing conclusions to be drawn about the overall efficiency of membrane mixing.

\subsubsection{Luminal contents are efficiently mixed upon fusion}

I developed a biochemical content-mixing assay using encapsulated His-SUMO-tagged sCPY*-GFP in one population of liposomes and and encapsulated SUMO protease (Ulp1) in another (Figure 3.15). This assay allowed me to monitor content mixing in substrate-containing liposomes and it also reported on the activity of the proteins encapsulated within. The SUMO tag was efficiently cleaved from the sCPY*-GFP in a SNARE-dependent manner, and cleavage was not observed when fusion was inhibited (Figure 3.15). I concluded from this experiment that the contents were efficiently mixed. Because sCPY*-GFP was cleaved by Ulp1, this shows that it was not in an aggregated state, which is a problem that I observed for $\mathrm{CPY}^{*}$ (see section 4.3.2 for detailed discussion).

It must be noted however, that I observed leakage of luminal contents during fusion, which may have contributed to the SNARE-dependent Ulp1 cleavage of substrate (Fig- 
ure 3.20, see section 4.1.2.3). For this reason, the luminal content mixing may not have been as efficient as expected.

\subsubsection{Leakage during fusion}

A potential issue with the fusion system is the leakage observed during fusion (Figures 3.20 and 3.21). Experiments with Doa10, which is not involved in ERAD-L, showed similar exposure of encapsulated sCPY*-GFP to the outside as Hrd1 (Figure 3.21). The leakage may be caused by high membrane protein concentration in the liposomes. Indeed, leakage during fusion has been previously observed in proteoliposomes containing SNARE:lipid densities of 1:1000 or higher (Dennison et al., 2006; Bhalla et al., 2006), indicating that high protein:lipid ratios destabilize membranes during fusion. Multipass transmembrane proteins like Hrd1 and Doa10 likely contribute more to membrane instability than single-TM containing SNAREs, at similar molar ratios. Leakage could be reduced by decreasing SNARE concentration (Bhalla et al., 2006; van den Bogaart et al., 2010). Therefore, an optimal concentration of Hrd1 and SNAREs that reduces leakage while maintaining fusion efficiency needs to be determined.

\subsection{Ubc6 functions as an E2 enzyme for Hrd1 in vitro}

The Ubc6 membrane mixing assay demonstrated that Ubc6 can act as an E2 enzyme for Hrd1 in a reconstituted system. Additionally, coreconstitution of Ubc6 with Hrd1 resulted in efficient autoubiquitination, even in the KRK mutant (Figure 3.45). This was somewhat surprising, because Ubc7 and Ubc1 were shown to be the E2s involved in ERAD-L and ERAD-M (Friedlander et al., 2000; Bays et al., 2001). However, one studied implicated Ubc6 as being partially required for $\mathrm{CPY}^{*}$ degradation (Hiller et al., 1996). I observed that the presence of Ubc6 resulted in short ubiquitin chains and more efficient Hrd1 autoubiquitination than with Ubc7 alone (Figures 3.14, 3.38). As the KRK mutant was efficiently autoubiquitinated in the presence of Ubc6 (Figure 3.45), Ubc6 likely primes Hrd1 on non-lysine residues for chain extension by Ubc7. This result is consistent with a previous observation that Ubc6 catalyzes monoubiquitination on lysines as well as on serine and threonine residues of Doa10 substrates (Weber et al., 2016). This monoubiquitination is thought to prime ERAD substrates for further polyubiquitination by Ubc7, much like the Ubc4 and Ubc1 combination that functions with the anaphase promoting complex (APC) (Rodrigo-Brenni and Morgan, 2007). One possible role for Ubc6 in the Hrd1 complex is the priming of substrates 
that have a low lysine content, as Ubc6 was absolutely required for degradation of an ERAD-C substrate that lacked lysine residues (Weber et al., 2016). Interestingly, Ube2J1, a mammalian homolog of Ubc6, is a core component of the mammalian Hrd1 complex and is required for ERAD-L (Christianson et al., 2011; Leto et al., 2019). The mammalian Hrd1 complex was also shown to ubiquitinate substrates on serine and threonine residues, presumably through Ube2J1 (Ishikura et al., 2010; Shimizu et al., 2010). Further experiments testing the role of Ubc6 in degradation of substrates lacking lysines are needed to provide greater insight into the role of Ubc6 in Hrd1-mediated ERAD.

\subsection{Potential issues with the fusion system}

Because the fusion was likely not the limiting factor in the fusion system, the question remains as to why encapsulated substrates were not efficiently retrotranslocated by Hrd1. This could have been caused by multiple factors, the most important of which are listed below:

1. Hrd1 was inactive or not in the correct oligomeric state.

2. The substrates were aggregated, oxidized, or not in a state conducive for retrotranslocation.

3. Retrotranslocation occurred but was very inefficient.

4. Hrd1 may be insufficient for retrotranslocation of luminal substrates.

These possibilities are discussed in detail in the following sections.

\subsubsection{Was Hrd1 inactive or in the incorrect oligomeric state?}

It is unlikely that Hrd1 activity was the problem because Hrd1 autoubiquitinated to acceptable levels (approximately 60\%) and the efficiency was independent of fusion (Figures 3.12, 3.24). Neither fusion nor the presence of SNAREs inhibited Hrd1 activity. Hrd1 autoubiquitination reached higher efficiencies when reconstituted with DMNG compared to DM, which was used in the fusion system (Figure 3.42A compared to Figure 3.12). However, when Hrd1 was reconstituted directly into CPY* liposomes with DMNG, its autoubiquitination efficiency was very high, yet CPY* was inefficiently ubiquitinated (Figure 3.26). 
It is possible that Hrd1 was not in the correct oligomeric state for retrotranslocation. In normal conditions, ERAD-L requires Usa1, which causes Hrd1 oligomerization and recruits Der1 (Horn et al., 2009; Carvalho et al., 2010). It is not clear if ERAD$\mathrm{L}$ requires a higher oligomeric state of $\mathrm{Hrd} 1$ for retrotranslocation. However, it has been shown that the ability for Hrd1 to compensate for the deletion of the other components of the complex depends on its overexpression levels. When Hrd1 is very highly overexpressed from a Gal1 promoter, CPY* is degraded with kinetics similar to the wild-type strain (Carvalho et al., 2010). When Hrd1 is only modestly overexpressed, it shows an intermediate compensation for Hrd3 deletion (Plemper et al., 1999; Denic et al., 2006; Vashistha et al., 2016). It may be that spontaneous Hrd1 oligomerization requires a higher concentration in the absence of Usa1. In vivo, this effect could be due to other factors, such as the decreased affinity for substrates in the absence of Hrd3. In any case, Hrd1 is already in a heterogenous oligomeric state after purification in DMNG (Stein et al., 2014). I used a protein to lipid ratio of 1:2000 in retrotranslocation experiments, identical to what was used in the study by Baldridge and Rapoport (2016). I did not use higher Hrd1 concentrations because of leakage during fusion, which appeared to be dependent on the concentration of transmembrane domains in the liposomes (Figure 3.21).

\subsubsection{Substrate aggregation}

It appears likely that CPY* and the other ERAD-L substrates used in the fusion system irreversibly aggregated during reconstitution. First, it is important to keep in mind that ERAD does not efficiently degrade aggregated proteins, highlighting the importance of chaperones in keeping misfolded proteins in a soluble state before retrotranslocation (Needham et al., 2019). The main indication of aggregation is that outside-bound

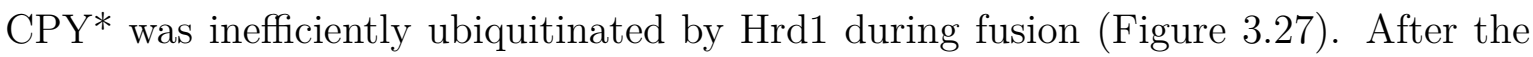
first step of the encapsulation protocol, $70 \%$ of $\mathrm{CPY}^{*}$ was accessible to proteinase $\mathrm{K}$, but only roughly $30 \%$ was ubiquitinated by Hrd1. In contrast, when CPY* and PrA* were added to the outside of Hrd1 liposomes, approximately $90 \%$ was ubiquitinated (Figure 3.34).

Experience in our lab indicates that $\mathrm{CPY}^{*}$ aggregates within a couple of hours after dilution from 2.5 M urea. For this reason, encapsulation was always performed in the presence of 1 or $2 \mathrm{M}$ urea. Reconstitution was performed as quickly as possible. However, because the reconstitution protocol for optimal $\Delta \mathrm{N}$ complex activity required dialysis for detergent removal, the time-factor could not be considerably shortened. 
Even with reconstitution in the presence of urea, CPY* was inefficiently ubiquitinated (Figures 3.16, 3.18). I attempted to resolubilize aggregated luminal CPY* by including $3 \mathrm{M}$ urea in the Nycodenz flotation (Figure 3.28). However, I did not observe any retrotranslocation in this case. It is not clear if the urea successfully penetrated into the lumen of liposomes or whether higher concentrations were required. Because PrA* behaved similarly to CPY* during purification, it likely also aggregated during encapsulation. The substrate H14-SUMO-sCPY*-GFP was apparently not in an aggregated state, because it could be cleaved by Ulp1 (Figure 3.15). However, it also was not ubiquitinated by $\operatorname{Hrd1}$, despite the fact that it leaked to the outside during fusion (Figure 3.19). The tightly-folded SUMO and GFP flanking the sCPY* domain likely preserved solubility, but the substrate may have aggregated after cleavage of the SUMO tag by Ulp1.

It is possible that in the presence of liposomes, the exposed hydrophobic regions in $\mathrm{CPY}^{*}$ and $\mathrm{PrA}^{*}$ insert like an amphipathic helix into the bilayer. The may bury the degron that is likely required for initiation of retrotranslocation and ubiquitination. Alternatively, the high protein concentration in the liposome lumen may cause irreversible aggregation. These factors may have contributed to why I was unable to encapsulate transmembrane domain-containing versions of CPY* (section 3.5.8).

\subsubsection{Ideas for overcoming substrate aggregation}

How can this be solved? Chemical chaperones such as glycerol or sucrose, or harsher denaturing agents like guanidine hydrochloride may help reduce aggregation. Additionally, chaperones such as the Hsp70 Kar2 and its Hsp40 cofactors, which have been shown to be required for CPY* degradation in vivo (Nishikawa et al., 2001), may keep substrates in a soluble state. One issue with Hsp70 chaperones is that their affinity for substrates is relatively low, with $K_{D}$ values in the micromolar range (Bukau et al., 2000). This may be overcome by using high concentrations of chaperone. Alternatively, it may be possible to co-encapsulate a His-tagged chaperone along with the substrate.

Another possibility is to use inducible misfolded substrates. The advantage would be that encapsulation and fusion can be performed with a well-folded, soluble protein. After fusion, the substrate would be induced to misfold in the lumen, where hopefully Hrd1 can capture it and initiate retrotranslocation before it has the chance to aggregate. An elegant system of inducible misfolding was developed by the Crews lab, using the modified bacterial dehalogenase protein (HaloTag), which forms a covalent bond with chloroalkane ligands (Los et al., 2008). They synthesized a ligand that causes 
proteasomal degradation of HaloTag fusion proteins in vivo, and induces the UPR when fused to an ER-localized protein (Neklesa et al., 2011; Raina et al., 2014). Most importantly, the ligand is membrane-permeable, which would allow it to access the lumen of liposomes. Of course, a prerequisite would be that the HaloTag has to become a Hrd1 substrate upon ligand binding.

As an alternative, temperature-sensitive mutants of soluble cytosolic proteins have been developed, which are degraded by the UPS upon shifting to non-permissive temperatures (Schneider et al., 2018). The temperature-sensitive form of Ubc9 (Ubc $9^{\text {ts }}$ ), has been shown to misfold at temperatures above $30{ }^{\circ} \mathrm{C}$ (Betting and Seufert, 1996; Kaganovich et al., 2008). Interestingly, Hrd1 and Doa10 are involved in its degradation in the cytosol upon misfolding (Samant et al., 2018). Because temperature shifting is very straight-forward and rapid, it could be applied to induce misfolding of encapsulated Ubc $9^{\text {ts }}$. It would have to be first tested whether ER-imported Ubc $9^{\text {ts }}$ is a Hrd1 substrate in vivo.

\subsubsection{Retrotranslocation may have occurred but was very inefficient}

Retrotranslocation experiments showed that approximately $25 \%$ of CPY*-H14 was ubiquitinated after fusion, while $18 \%$ was accessible to proteinase $\mathrm{K}$ before fusion (Figures 3.17 and 3.18). Only 15\% of CPY*-H14 was ubiquitinated in the inhibited fusion control. This means that $7-10 \%$ of CPY*-H14 was potentially retrotranslocated. It is possible that retrotranslocation is very inefficient after taking into account potential substrate aggregation and the lack of other components such as Der1. Further repetitions are required for more definitive conclusions. However, between experiments with slightly different reconstitution conditions, the amount of $\mathrm{CPY}^{*}$ protected from proteinase $\mathrm{K}$ ranged between $80-90 \%$, while ubiquitination after fusion was also variable, ranging between 10-20\%. Therefore, I focused my attention on improving retrotranslocation efficiency. Because I was interested in understanding mechanistic details about the process, I required a more efficient reaction.

\subsubsection{Is Hrd1 is insufficient for retrotranslocation?}

The final possibility is that Hrd1 is insufficient for efficient retrotranslocation of luminal substrates. Perhaps other components in the Hrd1 complex are required to efficiently insert substrates into the retrotranslocon. One strong candidate for this role is Der1, 
which interacts with luminal substrates with its transmembrane domains their way to Hrd1 (Carvalho et al., 2010; Mehnert et al., 2013). As Der1 is recruited to Hrd1 by Usa1 (Carvalho et al., 2006; Horn et al., 2009), a subcomplex of Hrd1/Usa1/Der1 would need to be reconstituted into the fusion system to test the effect of Der1 on retrotranslocation.

\subsection{Hrd1 forms an ion-conducting channel upon autoubiquitination}

When we incubated Hrd1 liposomes with ubiquitination machinery and added them to PLBs, we detected ion currents at different holding potentials (Figure 3.30), indicating that Hrd1 forms a channel. Autoubiquitination was clearly required for channel formation because Hrd1 liposomes incubated with ubiquitination mix lacking ATP did not fuse to the bilayer, nor did Hrd1 liposomes alone (Figure 3.30C). Additionally, the Hrd1 KRR mutant, which shows almost no autoubiquitination (Figures 3.41 and $3.42 \mathrm{~A}$ ), did not fuse to the lipid bilayer (Figure 3.44A). This result supports the previous observation that autoubiquitination is required for retrotranslocation of CPY*-TM in vitro (Baldridge and Rapoport, 2016). The channel on its own was dormant and small, because the gating events were relatively infrequent and the conductance states were in the range of $50 \mathrm{pS}$ (Figure 3.31D). Conductances in this regime correspond to a pore diameter of approximately $0.4 \mathrm{~nm}$, which is significantly smaller than the diameter of a single alpha helix (see materials and methods for details on calculating pore diameter). In order for Hrd1 to retrotranslocate substrates which may have folded domains with various topologies, we expected that it would need to open to a larger pore size. This is supported by previous electrophysiological measurements of the protein translocases Sec61, Tim23 and SecY, which translocate unfolded proteins across the ER membrane, inner mitochondrial membrane and bacterial inner membrane, respectively. These proteins show gating events with conductances in the range of 200-600 pS, which correspond to average pore diameters of approximately 1-2 nm (Wirth et al., 2003; Truscott et al., 2001; Saparov et al., 2007).

Strikingly, the addition of CPY* specifically to the luminal side of Hrd1 stimulated rapid channel gating, with conductances in the range of 200-600 pS observed (Figures $3.31,3.33)$. Thus, upon interaction with substrate, the Hrd1 pore expanded to a similar diameter as the aforementioned protein translocases. This result indicates that Hrd1 is dynamic, substrate-responsive channel. This would make sense, as it would 
be detrimental to have a constitutively open channel in the ER. We hypothesize that the expansion of the pore may correlate with substrate size, as was demonstrated for the peroxisomal importer Pex5/Pex14 (Meinecke et al., 2010). It was interesting that $\mathrm{CPY}^{*}$ addition stimulated further opening of the channel, as presequence addition to open Tim23 and Tom40 channels from mitochondria as well as the Toc75 channel from chloroplasts blocked the flow of ions (Truscott et al., 2001; Hill et al., 1998; Hinnah et al., 2002). It may reflect on the type of interaction of the substrate with the channel. CPY* may not completely insert into the channel. Despite the differences in channel conductance after substrate binding, CPY* increased Hrd1 gating frequency, an effect which has been observed with substrate binding to other translocation channels (Hill et al., 1998; Truscott et al., 2001; Kovermann et al., 2002). Importantly, we observed conductances in the range of $600 \mathrm{pS}$ without the presence of $\mathrm{CPY}^{*}$, but they were very rare (Figure 3.31D). This indicates that the non-stimulated channel has the ability to open to larger pore diameters, and that substrate binding possibly reduces the energy barrier for this further opening. A similar mechanism of activation energy reduction was proposed for signal sequence binding to the SecY channel (Gouridis et al., 2009).

What does the pore diameter tell us about retrotranslocation mechanism? The maximum conductance states we observed after CPY* addition were in the range of 800 pS (Figure 3.31D), which corresponds to pore diameters of roughly $2.5 \mathrm{~nm}$. This is certainly large enough to accommodate two alpha helices side by side, which would be expected if the substrate inserts as a loop, such as in translocation by SecY and Sec61 (Rapoport et al., 2017). This pore size indicates that larger folded domains would probably not be able to pass through the retrotranslocon, and would likely require Cdc48 for unfolding. However, the pore sizes from the theoretical calculations should be confirmed with an experimental approach such as the polymer-exclusion method (Krasilnikov et al., 1992).

\subsubsection{Channel closure by deubiquitination}

Remarkably, we observed that deubiquitination of Hrd1 irreversibly closed the channel (Figure 3.32). In the cell, a similar deubiquitination mechanism may inactivate Hrd1 channels after retrotranslocation is complete. The identity of the DUB responsible for this is still unknown. The DUB OTU1 (YOD1 in mammals) may be involved, as knockdown of YOD1 stimulated cholera toxin retrotranslocation (Bernardi et al., 2013). Interestingly, cholera toxin is not ubiquitinated but nevertheless requires Hrd1 for retrotranslocation, indicating that Hrd1 may be a target of YOD1 (Bernardi et al., 
2010). However, OTU1 normally functions with Cdc48 (Stein et al., 2014; Bodnar and Rapoport, 2017b), so this effect may have been connected to Cdc48 activity. USP19 is another DUB that was shown to be involved in mammalian ERAD and is localized to the ER membrane (Hassink et al., 2009). Overexpression of USP19 stabilized Hrd1 levels and was shown to reduce the accumulation of ubiquitinated Hrd1 (Harada et al., 2016). Deubiquitination of Hrd1 likely has to be tightly-regulated not just for channel closure, but because prolonged Hrd1 autoubiquitination may trigger its own degradation. Additionally, I raise the possibility that deubiquitination also serves to release the substrate from the cytoplasmic binding site, which may be especially important for nonubiquitinated substrates (Figure 3.40).

\subsection{Autoubiquitination in the Hrd1 RING domain creates a high affinity cytosolic binding site}

Hrd1 autoubiquitination led to binding of $\mathrm{CPY}^{*}$ and $\mathrm{PrA}^{*}$ to the cytoplasmic side of Hrd1 with high affinity (Figure 3.36). The binding was specific for misfolded proteins, as CPY WT bound inefficiently and PrA WT did not bind at all to autoubiquitinated Hrd1. Interestingly, both misfolded proteins did not bind to the cytoplasmic side of nonubiquitinated Hrd1 (Figure 3.36). Thus, it appears that a conformational change occurs upon Hrd1 autoubiquitination that creates a cytosolic binding site, which is normally occluded in the nonubiquitinated state. Cytosolic binding positions the substrate for efficient ubiquitination, because Hrd1 lysine mutants that bound CPY* with lower affinity ubiquitinated CPY* less efficiently (Figures 3.43 and Figures 3.41, 3.42). Using lysine to arginine mutants of $\mathrm{Hrd1}$, I identified that Hrd1 autoubiquitination in the RING domain was critical for high-affinity $\mathrm{CPY}^{*}$ binding and efficient CPY* polyubiquitination (Figure 3.43 for binding and Figures 3.41, 3.42 for ubiquitination). I observed that the RING domain lysines are the predominant attachment sites for long ubiquitin chains by Hrd1. This was evident because the overall autoubiquitination efficiency was not markedly different between Hrd1 mutants lacking lysines in the RING domain and mutants lacking lysines in the C-terminal extension (Figure 3.42A). However, the ubiquitin chains appeared longer when the RING domain lysines were present (Figure 3.41). Thus, Hrd1 autoubiquitinates across its entire cytoplasmic region, with short chains in the C-terminal extension and long chains in the RING domain. The 3K RING domain mutant, which was previously shown to be defective in retrotranslocation in vitro and $\mathrm{CPY}^{*}$ degradation in vivo (Baldridge and Rapoport, 2016), showed 
almost identical autoubiquitination efficiency and ubiquitin chain length as the KRK mutant (Figures 3.41, 3.42). Therefore, the primary ubiquitination sites in the RING domain are lysines 373,387 and 407. I hypothesize that reintroducing any one of these three lysines back into the KRK mutant would rescue Hrd1 autoubiquitination, substrate binding and substrate ubiquitination. This is based on the observation in the Baldridge study which showed that simply mutating combinations of two of these three lysines did not abolish CPY* degradation, indicating that they are redundant and can substitute for each other (Baldridge and Rapoport, 2016).

\subsection{Cytosolic binding site may provide the driving force in retrotranslocation}

What is the purpose of the cytosolic binding site? The intuitive reasoning is that substrate binding on the cytosolic side is a prerequisite for substrate ubiquitination, as E3 ligases need to bind to substrate to catalyze their ubiquitination (Deshaies and Joazeiro, 2009). However, I propose that an additional effect of the cytosolic substrate binding site is to provide the driving force during retrotranslocation. An overarching principle in protein translocation is that the translocon forms a passive pore in the membrane through which polypeptide segments diffuse by Brownian motion, and a driving force is necessary to provide directionality (Rapoport et al., 2017). In cotranslational protein translocation into the ER and across the bacterial plasma membrane, Sec61/SecY form the passive pore and the ribosome provides the driving force by preventing backsliding of the polypeptide into the cytosol as it is elongated through the channel (Rapoport et al., 2017; Connolly and Gilmore, 1986). In post translational translocation across the ER membrane, the luminal Hsp70 BiP acts as a Brownian ratchet to drive translocation of a polypeptide into the ER lumen (Matlack et al., 1999). It binds the polypeptide loosely in its ATP-bound state, after which the $\mathrm{J}$ domain of the accessory protein Sec63 stimulates ATP hydrolysis, causing BiP to bind more tightly to the translocating substrate. This tight association prevents backsliding into the channel. Through multiple cycles of binding and release by sequential BiP molecules, the polypeptide is ratcheted into the ER lumen (Matlack et al., 1999; Liebermeister et al., 2001). In post translational translocation across the bacterial plasma membrane, the SecA ATPase pushes segments of a translocating polypeptide into the SecY channel. SecA associates with the polypeptide in the ATP-bound state. Upon ATP hydrolysis, the polypeptide slides back and forth in the SecY channel until subsequent ADP-ATP exchange, and 
SecA in the ATP-bound form pushes the polypeptide further into the channel (Bauer et al., 2014; Rapoport et al., 2017). In the translocation of proteins from the cytosol into the mitochondrial matrix, the driving force through the Tim23 pore is provided by the membrane potential and the PAM motor in the matrix (Wiedemann and Pfanner, 2017). The PAM motor contains an Hsp70 protein (mtHsp70), a J protein and a nucleotide exchange factor. The mtHsp70 acts as a Brownian ratchet and may also provide a direct pulling force into the matrix (Wiedemann and Pfanner, 2017).

During Hrd1-mediated retrotranslocation, a polypeptide would be free to slide back and forth through Hrd1 unless it encounters a trap on the cytosolic side. The substrate binding site on the cytoplasmic side of Hrd1 would serve as this trap, preventing backsliding into the channel. Polyubiquitination of the substrate further prevents backsliding, but it mainly serves to recruit Cdc48, which hydrolyzes ATP to pull the substrate out of the membrane (Flierman et al., 2003) (see section 4.11 for model and further discussion).

\subsection{The nature of the conformational change in Hrd1}

Hrd1 autoubiquitination appears to cause a conformational change, and our results show that this conformational change is most efficiently stabilized when Hrd1 autoubiquitinates in its RING domain. Consistent with this interpretation was the observation that the Hrd1 KRK mutant was able to form channels, but these channels were extremely short-lived and unstable (Figure 3.44). In our experiments, we were unable to uncouple the conformational change leading to channel formation from that which causes cytosolic substrate binding. Both experiments may have indeed been reporting on the same conformational change, which serves multiple purposes: opening the channel and providing a cytosolic binding site as the driving force for retrotranslocation. It is possible that the combination of channel instability coupled with inefficient substrate binding on the cytosolic side contributes to a defect in ERAD-L when lysines are not present in the RING domain. These results may provide an explanation to the ERAD-

$\mathrm{L}$ defect that was previously observed with the KRK and 3K mutants (Baldridge and Rapoport, 2016; Neal et al., 2018).

The nature of the conformational change in Hrd1 upon autoubiquitination remains mysterious. The Hrd1/Hrd3 cryo-EM structure does not show a density for the RING domain, indicating that it is part of a flexible region (Schoebel et al., 2017). However, the RING domain is located closer to the last transmembrane domain than previously 
appreciated, due to the fact that Hrd1 was shown to have eight TMs instead of six (Deak and Wolf, 2001; Schoebel et al., 2017). The RING domain is approximately 25 amino acids away from the last TM, indicating that it may be in proximity to affect the conformation of the transmembrane domains of Hrd1 after autoubiquitination. A recent NMR structure of the Hrd1 RING domain from $S$. cerevisiae shows that the three primary acceptor lysines in the RING domain (K373, K387 and K407) all face away from the canonical E2 binding site, close to the C-terminus of the RING domain (Kniss et al., 2019) (Figure 4.1). The four other lysines in the Hrd1 RING domain, which are not required for ERAD-L (Baldridge and Rapoport, 2016), are located in a loop and beta strand before the three primary acceptor lysines, facing $90^{\circ}$ away from them. It is not clear why the three terminal lysines are preferentially ubiquitinated, but the structure explains why they can substitute each for other, as they are all located on the same side of the RING domain and are perhaps better positioned for ubiquitin transfer after E2 binding.
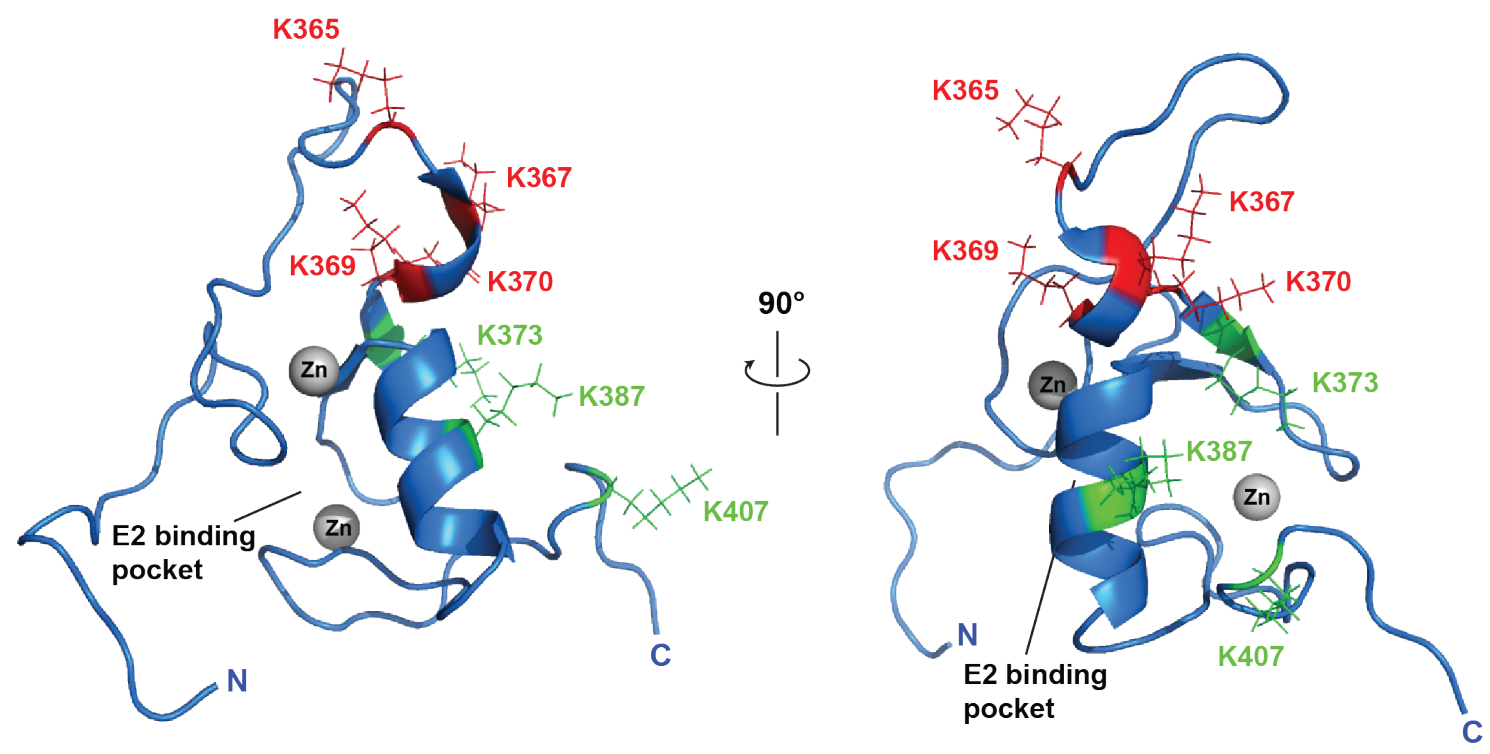

Figure 4.1: NMR Stucture of the Hrd1 RING domain

Structure created with PyMOL from PDB ID 6F98 (Kniss et al., 2019). Zinc ions that coordinate conserved cysteine and histidine residues are indicated. The canonical E2 binding pocket was derived from the structure of the c-Cbl E3 ligase in complex with the E2 UbcH7 (Zheng et al., 2000), with additional input from alignments in Deshaies and Joazeiro (2009).

Interestingly, homologs of Hrd1 in other species have significantly fewer lysines in the cytosolic region than in S. cerevisiae. K373 from S. cerevisiae is highly conserved, and in some species, this is the only lysine present in the RING domain (Baldridge and 
Rapoport, 2016). This indicates that K373 is probably sufficient for channel formation and cytosolic substrate binding. The other lysines may have been lost to minimize nonspecific autoubiquitination and degradation, as seems to be the case in other RING E3s (Boomsma et al., 2016).

\subsubsection{Hrd1 autoubiquitination may facilitate monomerization}

One idea is that autoubiquitination might facilitate dissociation of the Hrd1 dimers or oligomers into monomers. In this case, the dimer found in the Hrd1/Hrd3 structure would be the inactive form (Schoebel et al., 2017). This is supported by in vivo evidence that a retrotranslocating ERAD-L substrate crosslinked to Hrd1 efficiently even in the absence of Usa1, which is required for Hrd1 oligomerization (Carvalho et al., 2010). Other translocation channels share a monomerization mechanism. Tim23 is normally present as a dimer and it dissociates upon presequence binding into the active monomeric channel (Bauer et al., 1996). SecY is a dimer that contains only one active pore, with the other SecY molecule involved in targeting the polypeptide into the active channel (Osborne and Rapoport, 2007). Autoubiquitination of Hrd1 may similarly lead to monomerization.

In the cell, Hrd1 is present in a multi-subunit complex, whose components likely regulate its autoubiquitination. It is logical that Hrd1 only autoubiquitinate when substrate is present. Hrd3 may regulate Hrd1 autoubiquitination by linking it to substrate binding. It interacts with substrate upstream of Hrd1 and binds to the loop between TMs 1 and 2 of Hrd1 (Carvalho et al., 2010; Schoebel et al., 2017). In the absence of Hrd3, Hrd1 autoubiquitination levels increase and Hrd1 is rapidly degraded (Gardner et al., 2000). Recent evidence shows that Hrd3 may directly influence Hrd1 ubiquitination activity (Vashistha et al., 2016). Additionally, Usa1 binds to the C-terminus of Hrd1 and may block access to ubiquitination sites in the C-terminal extension. Accordingly, Hrd3 may block autoubiquitination of the Hrd1 RING domain until substrate binding, where it would release this inhibition.

\subsection{Possible mechanisms of cytosolic substrate binding by Hrd1}

An important question remaining is what forms the cytoplasmic substrate binding site in Hrd1? It is unlikely that a misfolded protein binds to the RING domain itself, as 
RING domains are globular, soluble domains (Zheng et al., 2000). Interestingly, apart from the RING domain, the Hrd1 C-terminal region is largely unstructured and is predicted to be intrinsically disordered (Schulz et al., 2017). Other E3 ubiquitin ligases, like the nuclear-localized San1 in yeast, bind substrates directly through intrinsicallydisordered regions flanked by short ordered regions (Rosenbaum et al., 2011; Boomsma et al., 2016). This is thought to provide a broad spectrum of substrate specificity, which is especially important in ERAD, as substrates do not have defined degrons. Perhaps Hrd1 autoubiquitination triggers a conformational change which leads to the formation of hydrophobic pocket or clamp, which may be surrounded by intrinsically disordered domains.

It is difficult to completely rule out that the substrate binding is contributed by long poylubiquitin chains attached to Hrd1. Although I observed no CPY* binding to polyubiquitinated Ubc6, the chain length appeared to shorter than the chains on Hrd1 (Figure 3.39). Ubiquitin contains a hydrophobic patch which normally interacts with ubiquitin binding domains (Winget and Mayor, 2010). It is possible that long chains create a distributed hydrophobic binding surface for misfolded proteins. It could be that a combination of the aforementioned mechanisms contributes to overall substrate affinity upon Hrd1 autoubiquitination. Autoubiquitination as a mechanism to increase substrate affinity has been observed in other E3 ligases such as TRAF6 and NEDD4 (de Bie and Ciechanover, 2011), indicating that it may be a conserved mechanism.

\subsection{Autoubiquitination of $\mathrm{Hrd1}$ as an allosteric post-translational modification}

Autoubiquitination of Hrd1 induces a conformational change leading to channel activity (Figure 3.30), and this modification is completely reversible (Figure 3.32). Additionally, cytosolic substrate binding was induced by autoubiquitination and was reversible, as $\mathrm{CPY}^{*}$ was partially released after deubiquitination (Figure 3.40). These results imply that Hrd1 autoubiquitination acts as an allosteric post-translational modification, affecting channel activity and cytosolic binding from an external site. Interestingly, ubiquitination is rarely utilized as an allosteric modulator, unlike phosphorylation, which is prevalent in many processes (Nussinov et al., 2012). Autoubiquitination is not uncommon in E3 ligases, and is usually a self-regulation mechanism, resulting in proteasomal degradation (de Bie and Ciechanover, 2011). Autoubiquitination of E3 ligases has also been shown to increase ubiquitination activity and recruit sub- 
strates (de Bie and Ciechanover, 2011), a mechanism which may have commonality with Hrd1 cytosolic substrate binding. What makes the allosteric modification of Hrd1 by autoubiquitination a unique case is that it affects channel activity in addition to substrate binding and ubiquitination. One example in the literature shows a similar situation. The epithelial sodium channel $\mathrm{ENaC}$ is partly-regulated by extracellular proteolysis. Ubiquitination of the channel on the cytosolic side causes it to adopt a more tightly-folded conformation on the extracellular side, making it more resistant to extracellular proteases (Ruffieux-Daidié and Staub, 2010). This supports the idea that ubiquitination can result in conformational changes across the membrane.

As monoubiquitination resulted neither in channel activity (Figure 3.44) nor cytosolic substrate binding (Figure 3.37), one question that remains is what is the minimal ubiquitin chain length for these effects? Because loading of presynthesized ubiquitin chains of varying lengths onto the E1 enzyme was inefficient (data not shown), one idea to synthesize shorter chains is to use a mutant of Cue1 lacking the CUE domain, as has been previously demonstrated (Bagola et al., 2013). Another idea is to titrate low concentrations of Ubc7 and Cue1, and to stop the ubiquitination reaction with a zinc chelator after short timepoints. Knowledge of the minimum ubiquitin chain length will help in deducing the nature of the conformational change.

\subsection{Substrate binding on the luminal side of Hrd1}

Hrd1 contains a luminal binding site for misfolded proteins, because CPY*, but not CPY WT, bound to nonubiquitinated Hrd1 in nanodiscs (Figure 3.52), but not in lipsomes (Figure 3.36). As this is an indirect determination of luminal binding, detection of direct substrate binding to the luminal side of Hrd1 awaits site-specific crosslinking and FRET experiments. This is the first time substrate binding has been detected on the luminal side of Hrd1 in a reconstituted system. It offers an explanation as to why overexpressed Hrd1 compensates for the deletion of other ERAD components, including the substrate receptor Hrd3 (Carvalho et al., 2010). Hrd1 has a weaker affinity for $\mathrm{CPY}^{*}$ on its luminal side in the nonubiquitinated state than on the cytosolic side in its autoubiquitinated state, which may contribute to the driving force in retrotranslocation by an affinity gradient mechanism (Figure 4.2).

We observed CPY* activation of the channel on the luminal side of ubiquitinated Hrd1 (Figures $3.33,3.31$ ). Since it is difficult to differentiate cytosolic from luminal binding to ubiquitinated Hrd1 in nanodiscs, it is unclear what the substrate affinity for the 
luminal side of Hrd1 is in the autoubiquitinated state. Another question that arises is where substrates bind on the luminal side, as the structure shows mainly loops on that side of Hrd1 (Schoebel et al., 2017) (Figure 1.6). Perhaps a combination of weak interactions with luminal loops of multiple Hrd1 molecules contributes to the overall affinity. Finally, it will also be interesting to test the luminal binding of substrates to the Hrd1/Hrd3 complex in nanodiscs, particularly in combination with subsequent autoubiquitination experiments.

\subsection{Proposed mechanism of retrotranslocation by Hrd1}

Based on the evidence presented in this thesis, an updated model of retrotranslocation of misfolded luminal proteins by Hrd1 is presented in Figure 4.2. In step 1, the substrate binds to the luminal side of Hrd1. Autoubiquitination of Hrd1 on lysine residues in its RING domain leads to channel opening (step 2). Substrate binding to the luminal side stimulates channel gating and expansion (step 2). Subsequently, the substrate inserts in to the channel and is free to slide back and forth until it encounters the substrate binding site, which locks the substrate into place and prevents backsliding (step 3). The substrate is polyubiquitinated by Hrd1 on the cytosolic side of the membrane, which in turn recruits the Cdc48 complex (step 4). The Cdc48 complex hydrolyzes ATP to extract the substrate out of the membrane. Finally, a DUB removes ubiquitin chains from Hrd1, which closes the channel.

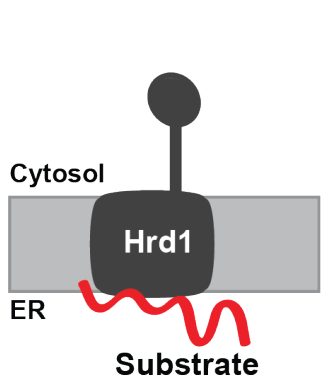

1

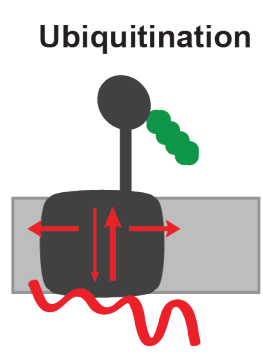

2

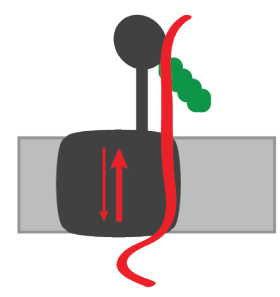

3

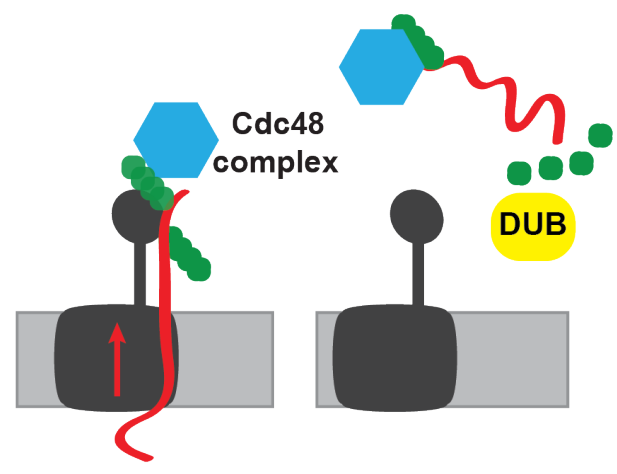

5

Figure 4.2: Updated mechanism of retrotranslocation by Hrd1

Proposed mechanism of retrotranslocation of a luminal misfolded protein by Hrd1. See text for details. 



\section{Bibliography}

Adamala, K. P., Martin-Alarcon, D. A., Guthrie-Honea, K. R. and Boyden, E. S. (2016). Engineering genetic circuit interactions within and between synthetic minimal cells. Nature Chemistry 9, 431-439.

Akimaru, J., ichi Matsuyama, S., Tokuda, H. and Mizushima, S. (1991). Reconstitution of a protein translocation system containing purified SecY, SecE, and SecA from Escherichia coli. Proceedings of the National Academy of Sciences 88, 6545-6549.

Antharavally, B. S., Mallia, K. A., Rosenblatt, M. M., Salunkhe, A. M., Rogers, J. C., Haney, P. and Haghdoost, N. (2011). Efficient removal of detergents from proteins and peptides in a spin column format. Analytical Biochemistry 416, 39-44.

Bagola, K., von Delbrueck, M., Dittmar, G., Scheffner, M., Ziv, I., Glickman, M. H., Ciechanover, A. and Sommer, T. (2013). Ubiquitin Binding by a CUE Domain Regulates Ubiquitin Chain Formation by ERAD E3 Ligases. Molecular Cell 50, 528-539.

Balchin, D., Hayer-Hartl, M. and Hartl, F. U. (2016). In vivo aspects of protein folding and quality control. Science 353, aac4354.

Baldridge, R. D. and Rapoport, T. A. (2016). Autoubiquitination of the Hrd1 Ligase Triggers Protein Retrotranslocation in ERAD. Cell 166, 394-407.

Ballweg, S. and Ernst, R. (2017). Control of membrane fluidity: the OLE pathway in focus. Biological Chemistry 398.

Banerjee, S., Bartesaghi, A., Merk, A., Rao, P., Bulfer, S. L., Yan, Y., Green, N., Mroczkowski, B., Neitz, R. J., Wipf, P., Falconieri, V., Deshaies, R. J., Milne, J. L. S., Huryn, D., Arkin, M. and Subramaniam, S. (2016). 2.3 A resolution cryo-EM structure of human p97 and mechanism of allosteric inhibition. Science 351, 871-875.

Bard, J. A., Goodall, E. A., Greene, E. R., Jonsson, E., Dong, K. C. and Martin, A. (2018). Structure and Function of the 26S Proteasome. Annual Review of Biochemistry $87,697-724$. 
Bauer, B. W., Shemesh, T., Chen, Y. and Rapoport, T. A. (2014). A "Push and Slide" Mechanism Allows Sequence-Insensitive Translocation of Secretory Proteins by the SecA ATPase. Cell 15\%, 1416-1429.

Bauer, M. F., Sirrenberg, C., Neupert, W. and Brunner, M. (1996). Role of Tim23 as Voltage Sensor and Presequence Receptor in Protein Import into Mitochondria. Cell $87,33-41$.

Bays, N. W., Gardner, R. G., Seelig, L. P., Joazeiro, C. A. and Hampton, R. Y. (2001). Hrd1p/Der3p is a membrane-anchored ubiquitin ligase required for ER-associated degradation. Nature Cell Biology 3, 24-29.

Bazirgan, O. A., Garza, R. M. and Hampton, R. Y. (2006). Determinants of RING-E2 Fidelity for Hrd1p, a Membrane-anchored Ubiquitin Ligase. Journal of Biological Chemistry 281, 38989-39001.

Bazirgan, O. A. and Hampton, R. Y. (2008). Cue1p Is an Activator of Ubc7p E2 Activity in Vitro and in Vivo. Journal of Biological Chemistry 283, 12797-12810.

Bello, O. D., Auclair, S. M., Rothman, J. E. and Krishnakumar, S. S. (2016). Using ApoE Nanolipoprotein Particles To Analyze SNARE-Induced Fusion Pores. Langmuir 32, 3015-3023.

Bernardi, K. M., Williams, J. M., Inoue, T., Schultz, A. and Tsai, B. (2013). A deubiquitinase negatively regulates retro-translocation of nonubiquitinated substrates. Molecular Biology of the Cell 24, 3545-3556.

Bernardi, K. M., Williams, J. M., Kikkert, M., van Voorden, S., Wiertz, E. J., Ye, Y. and Tsai, B. (2010). The E3 Ubiquitin Ligases Hrd1 and gp78 Bind to and Promote Cholera Toxin Retro-Translocation. Molecular Biology of the Cell 21, 140-151.

Berner, N., Reutter, K.-R. and Wolf, D. H. (2018). Protein Quality Control of the Endoplasmic Reticulum and Ubiquitin-Proteasome-Triggered Degradation of Aberrant Proteins: Yeast Pioneers the Path. Annual Review of Biochemistry 87, 751-782.

Betting, J. and Seufert, W. (1996). A Yeast Ubc9 Mutant Protein with Temperaturesensitive in VivoFunction Is Subject to Conditional Proteolysis by a Ubiquitin- and Proteasome-dependent Pathway. Journal of Biological Chemistry 271, 25790-25796.

Bhalla, A., Chicka, M. C., Tucker, W. C. and Chapman, E. R. (2006). Ca2-synaptotagmin directly regulates t-SNARE function during reconstituted membrane fusion. Nature Structural \& Molecular Biology 13, 323-330.

Bhamidipati, A., Denic, V., Quan, E. M. and Weissman, J. S. (2005). Exploration 
of the Topological Requirements of ERAD Identifies Yos9p as a Lectin Sensor of Misfolded Glycoproteins in the ER Lumen. Molecular Cell 19, 741-751.

Biederer, T., Volkwein, C. and Sommer, T. (1997). Role of Cue1p in Ubiquitination and Degradation at the ER Surface. Science 278, 1806-1809.

Bodnar, N. and Rapoport, T. (2017a). Toward an understanding of the Cdc48/p97 ATPase. F1000Research 6, 1318.

Bodnar, N. O. and Rapoport, T. A. (2017b). Molecular Mechanism of Substrate Processing by the Cdc48 ATPase Complex. Cell 169, 722-735.e9.

Bonifacino, J. S. and Glick, B. S. (2004). The Mechanisms of Vesicle Budding and Fusion. Cell 116, 153-166.

Boomsma, W., Nielsen, S. V., Lindorff-Larsen, K., Hartmann-Petersen, R. and Ellgaard, L. (2016). Bioinformatics analysis identifies several intrinsically disordered human E3 ubiquitin-protein ligases. PeerJ 4, e1725.

Bordallo, J., Plemper, R. K., Finger, A. and Wolf, D. H. (1998). Der3p/Hrd1p Is Required for Endoplasmic Reticulum-associated Degradation of Misfolded Lumenal and Integral Membrane Proteins. Molecular Biology of the Cell 9, 209-222.

Bordallo, J. and Wolf, D. H. (1999). A RING-H2 finger motif is essential for the function of Der3/Hrd1 in endoplasmic reticulum associated protein degradation in the yeast Saccharomyces cerevisiae. FEBS Letters 448, 244-248.

Braakman, I. and Hebert, D. N. (2013). Protein Folding in the Endoplasmic Reticulum. Cold Spring Harbor Perspectives in Biology 5, a013201-a013201.

Brandman, O., Stewart-Ornstein, J., Wong, D., Larson, A., Williams, C. C., Li, G.-W., Zhou, S., King, D., Shen, P. S., Weibezahn, J., Dunn, J. G., Rouskin, S., Inada, T., Frost, A. and Weissman, J. S. (2012). A Ribosome-Bound Quality Control Complex Triggers Degradation of Nascent Peptides and Signals Translation Stress. Cell 151, 1042-1054.

Brodsky, J. L., Werner, E. D., Dubas, M. E., Goeckeler, J. L., Kruse, K. B. and McCracken, A. A. (1999). The Requirement for Molecular Chaperones during Endoplasmic Reticulum-associated Protein Degradation Demonstrates That Protein Export and Import Are Mechanistically Distinct. Journal of Biological Chemistry 274, 3453-3460.

Brundage, L., Hendrick, J. P., Schiebel, E., Driessen, A. J. and Wickner, W. (1990). The purified E. coli integral membrane protein SecYE is sufficient for reconstitution of SecA-dependent precursor protein translocation. Cell 62, 649-657. 
Bukau, B., Mayer, M. P., Schroeder, H., Ruediger, S., Paal, K. and Laufen, T. (2000). Multistep mechanism of substrate binding determines chaperone activity of Hsp70. Nature Structural Biology 7, 586-593.

Burke, D. J., Amberg, D. C. and Strathern, J. N. (2005). Methods in Yeast Genetics: A Cold Spring Harbor Laboratory Course Manual, 2005 Edition. Cold Spring Harbor Laboratory Press.

Buschhorn, B. A., Kostova, Z., Medicherla, B. and Wolf, D. H. (2004). A genome-wide screen identifies Yos9p as essential for ER-associated degradation of glycoproteins. FEBS Letters $577,422-426$.

Carroll, S. M. and Hampton, R. Y. (2010). Usa1p Is Required for Optimal Function and Regulation of the Hrd1p Endoplasmic Reticulum-associated Degradation Ubiquitin Ligase. Journal of Biological Chemistry 285, 5146-5156.

Carvalho, P., Goder, V. and Rapoport, T. A. (2006). Distinct Ubiquitin-Ligase Complexes Define Convergent Pathways for the Degradation of ER Proteins. Cell 126, $361-373$.

Carvalho, P., Stanley, A. M. and Rapoport, T. A. (2010). Retrotranslocation of a Misfolded Luminal ER Protein by the Ubiquitin-Ligase Hrd1p. Cell 143, 579-591.

Chau, V., Tobias, J., Bachmair, A., Marriott, D., Ecker, D., Gonda, D. and Varshavsky, A. (1989). A multiubiquitin chain is confined to specific lysine in a targeted shortlived protein. Science 243, 1576-1583.

Chen, I., Dorr, B. M. and Liu, D. R. (2011). A general strategy for the evolution of bond-forming enzymes using yeast display. Proceedings of the National Academy of Sciences 108, 11399-11404.

Christianson, J. C., Olzmann, J. A., Shaler, T. A., Sowa, M. E., Bennett, E. J., Richter, C. M., Tyler, R. E., Greenblatt, E. J., Harper, J. W. and Kopito, R. R. (2011). Defining human ERAD networks through an integrative mapping strategy. Nature Cell Biology 14, 93-105.

Christianson, J. C., Shaler, T. A., Tyler, R. E. and Kopito, R. R. (2008). OS-9 and GRP94 deliver mutant 1-antitrypsin to the Hrd1-SEL1L ubiquitin ligase complex for ERAD. Nature Cell Biology 10, 272-282.

Christianson, J. C. and Ye, Y. (2014). Cleaning up in the endoplasmic reticulum: ubiquitin in charge. Nature Structural \& Molecular Biology 21, 325-335.

Chromy, B. A., Arroyo, E., Blanchette, C. D., Bench, G., Benner, H., Cappuccio, J. A., Coleman, M. A., Henderson, P. T., Hinz, A. K., Kuhn, E. A., Pesavento, 
J. B., Segelke, B. W., Sulchek, T. A., Tarasow, T., Walsworth, V. L. and Hoeprich, P. D. (2007). Different Apolipoproteins Impact Nanolipoprotein Particle Formation. Journal of the American Chemical Society 129, 14348-14354.

Clerc, S., Hirsch, C., Oggier, D. M., Deprez, P., Jakob, C., Sommer, T. and Aebi, M. (2009). Htm1 protein generates the N-glycan signal for glycoprotein degradation in the endoplasmic reticulum. The Journal of Cell Biology 184, 159-172.

Cohen, F. S., Zimmerberg, J. and Finkelstein, A. (1980). Fusion of phospholipid vesicles with planar phospholipid bilayer membranes. II. Incorporation of a vesicular membrane marker into the planar membrane. The Journal of General Physiology $75,251-270$.

Colletier, J.-P., Chaize, B., Winterhalter, M. and Fournier, D. (2002). Protein encapsulation in liposomes: efficiency depends on interactions between protein and phospholipid bilayer. BMC biotechnology 2, 9 .

Connolly, T. and Gilmore, R. (1986). Formation of a functional ribosome-membrane junction during translocation requires the participation of a GTP-binding protein. The Journal of Cell Biology 103, 2253-2261.

Dancourt, J. and Barlowe, C. (2010). Protein Sorting Receptors in the Early Secretory Pathway. Annual Review of Biochemistry 79, 777-802.

de Bie, P. and Ciechanover, A. (2011). Ubiquitination of E3 ligases: self-regulation of the ubiquitin system via proteolytic and non-proteolytic mechanisms. Cell Death \& Differentiation 18, 1393-1402.

Deak, P. M. and Wolf, D. H. (2001). Membrane Topology and Function of Der3/Hrd1p as a Ubiquitin-Protein Ligase (E3) Involved in Endoplasmic Reticulum Degradation. Journal of Biological Chemistry 276, 10663-10669.

Denic, V., Quan, E. M. and Weissman, J. S. (2006). A Luminal Surveillance Complex that Selects Misfolded Glycoproteins for ER-Associated Degradation. Cell 126, 349359.

Denisov, I. G. and Sligar, S. G. (2017). Nanodiscs in Membrane Biochemistry and Biophysics. Chemical Reviews 117, 4669-4713.

Denkert, N., Schendzielorz, A. B., Barbot, M., Versemann, L., Richter, F., Rehling, P. and Meinecke, M. (2017). Cation selectivity of the presequence translocase channel Tim23 is crucial for efficient protein import. eLife 6 .

Dennison, S. M., Bowen, M. E., Brunger, A. T. and Lentz, B. R. (2006). Neuronal 
SNAREs Do Not Trigger Fusion between Synthetic Membranes but Do Promote PEG-Mediated Membrane Fusion. Biophysical Journal 90, 1661-1675.

Deshaies, R. J. and Joazeiro, C. A. (2009). RING Domain E3 Ubiquitin Ligases. Annual Review of Biochemistry 78, 399-434.

Diao, J., Su, Z., Ishitsuka, Y., Lu, B., Lee, K. S., Lai, Y., Shin, Y.-K. and Ha, T. (2010). A single-vesicle content mixing assay for SNARE-mediated membrane fusion. Nature Communications 1, 1-6.

Dyballa, N. and Metzger, S. (2009). Fast and Sensitive Colloidal Coomassie G-250 Staining for Proteins in Polyacrylamide Gels. Journal of Visualized Experiments 3.

Ellgaard, L. and Helenius, A. (2003). Quality control in the endoplasmic reticulum. Nature Reviews Molecular Cell Biology 4, 181-191.

Ernst, R., Mueller, B., Ploegh, H. L. and Schlieker, C. (2009). The Otubain YOD1 Is a Deubiquitinating Enzyme that Associates with p97 to Facilitate Protein Dislocation from the ER. Molecular Cell 36, 28-38.

Fasshauer, D., Otto, H., Eliason, W. K., Jahn, R. and Brunger, A. T. (1997). Structural Changes Are Associated with SolubleN-Ethylmaleimide-sensitive Fusion Protein Attachment Protein Receptor Complex Formation. Journal of Biological Chemistry 272, 28036-28041.

Finger, A., Knop, M. and Wolf, D. H. (1993). Analysis of two mutated vacuolar proteins reveals a degradation pathway in the endoplasmic reticulum or a related compartment of yeast. European Journal of Biochemistry 218, 565-574.

Finley, D. (2009). Recognition and Processing of Ubiquitin-Protein Conjugates by the Proteasome. Annual Review of Biochemistry 78, 477-513.

Finley, D., Ulrich, H. D., Sommer, T. and Kaiser, P. (2012). The Ubiquitin-Proteasome System of Saccharomyces cerevisiae. Genetics 192, 319-360.

Flierman, D., Ye, Y., Dai, M., Chau, V. and Rapoport, T. A. (2003). Polyubiquitin Serves as a Recognition Signal, Rather than a Ratcheting Molecule, during Retrotranslocation of Proteins across the Endoplasmic Reticulum Membrane. Journal of Biological Chemistry 278, 34774-34782.

Flury, I., Garza, R., Shearer, A., Rosen, J., Cronin, S. and Hampton, R. Y. (2005). INSIG: a broadly conserved transmembrane chaperone for sterol-sensing domain proteins. The EMBO Journal 24, 3917-3926. 
Franken, L. E., Boekema, E. J. and Stuart, M. C. A. (2017). Transmission Electron Microscopy as a Tool for the Characterization of Soft Materials: Application and Interpretation. Advanced Science 4, 1600476.

Freeman, M. (2014). The Rhomboid-Like Superfamily: Molecular Mechanisms and Biological Roles. Annual Review of Cell and Developmental Biology 30, 235-254.

Frey, S. and Gorlich, D. (2014). A new set of highly efficient, tag-cleaving proteases for purifying recombinant proteins. Journal of Chromatography A 1337, 95-105.

Friedlander, R., Jarosch, E., Urban, J., Volkwein, C. and Sommer, T. (2000). A regulatory link between ER-associated protein degradation and the unfolded-protein response. Nature Cell Biology 2, 379-384.

Gardner, R. G., Shearer, A. G. and Hampton, R. Y. (2001). In Vivo Action of the HRD Ubiquitin Ligase Complex: Mechanisms of Endoplasmic Reticulum Quality Control and Sterol Regulation. Molecular and Cellular Biology 21, 4276-4291.

Gardner, R. G., Swarbrick, G. M., Bays, N. W., Cronin, S. R., Wilhovsky, S., Seelig, L., Kim, C. and Hampton, R. Y. (2000). Endoplasmic Reticulum Degradation Requires Lumen to Cytosol Signaling. The Journal of Cell Biology 151, 69-82.

Garza, R. M., Sato, B. K. and Hampton, R. Y. (2009). In VitroAnalysis of Hrd1pmediated Retrotranslocation of Its Multispanning Membrane Substrate 3-Hydroxy-3methylglutaryl (HMG)-CoA Reductase. Journal of Biological Chemistry 284, 1471014722 .

Gauss, R., Jarosch, E., Sommer, T. and Hirsch, C. (2006). A complex of Yos9p and the HRD ligase integrates endoplasmic reticulum quality control into the degradation machinery. Nature Cell Biology 8, 849-854.

Gauss, R., Kanehara, K., Carvalho, P., Ng, D. T. and Aebi, M. (2011). A Complex of Pdi1p and the Mannosidase Htm1p Initiates Clearance of Unfolded Glycoproteins from the Endoplasmic Reticulum. Molecular Cell 42, 782-793.

Ghaemmaghami, S., Huh, W.-K., Bower, K., Howson, R. W., Belle, A., Dephoure, N., O'Shea, E. K. and Weissman, J. S. (2003). Global analysis of protein expression in yeast. Nature 425, 737-741.

Goder, V., Carvalho, P. and Rapoport, T. A. (2008). The ER-associated degradation component Der1p and its homolog Dfm1p are contained in complexes with distinct cofactors of the ATPase Cdc48p. FEBS Letters 582, 1575-1580.

Gorlich, D. and Rapoport, T. A. (1993). Protein translocation into proteoliposomes 
reconstituted from purified components of the endoplasmic reticulum membrane. Cell $75,615-630$.

Gouridis, G., Karamanou, S., Gelis, I., Kalodimos, C. G. and Economou, A. (2009). Signal peptides are allosteric activators of the protein translocase. Nature 462, 363-367.

Greenblatt, E. J., Olzmann, J. A. and Kopito, R. R. (2011). Derlin-1 is a rhomboid pseudoprotease required for the dislocation of mutant -1 antitrypsin from the endoplasmic reticulum. Nature Structural \& Molecular Biology 18, 1147-1152.

Grinkova, Y. V., Denisov, I. G. and Sligar, S. G. (2010). Engineering extended membrane scaffold proteins for self-assembly of soluble nanoscale lipid bilayers. Protein Engineering Design and Selection 23, 843-848.

Guerriero, C. J. and Brodsky, J. L. (2012). The Delicate Balance Between Secreted Protein Folding and Endoplasmic Reticulum-Associated Degradation in Human Physiology. Physiological Reviews 92, 537-576.

Habeck, G., Ebner, F. A., Shimada-Kreft, H. and Kreft, S. G. (2015). The yeast ERAD-C ubiquitin ligase Doa10 recognizes an intramembrane degron. The Journal of Cell Biology 209, 261-273.

Hagan, C. L., Kim, S. and Kahne, D. (2010). Reconstitution of Outer Membrane Protein Assembly from Purified Components. Science 328, 890-892.

Hampton, R. Y., Gardner, R. G. and Rine, J. (1996). Role of 26S proteasome and HRD genes in the degradation of 3-hydroxy-3-methylglutaryl-CoA reductase, an integral endoplasmic reticulum membrane protein. Molecular Biology of the Cell 7, 20292044 .

Hampton, R. Y. and Sommer, T. (2012). Finding the will and the way of ERAD substrate retrotranslocation. Current Opinion in Cell Biology 24, 460-466.

Hanson, P. I. and Whiteheart, S. W. (2005). AAA proteins: have engine, will work. Nature Reviews Molecular Cell Biology 6, 519-529.

Harada, K., Kato, M. and Nakamura, N. (2016). USP19-Mediated Deubiquitination Facilitates the Stabilization of HRD1 Ubiquitin Ligase. International Journal of Molecular Sciences 17, 1829.

Harsman, A., Bartsch, P., Hemmis, B., Krueger, V. and Wagner, R. (2011). Exploring protein import pores of cellular organelles at the single molecule level using the planar lipid bilayer technique. European Journal of Cell Biology 90, 721-730. 
Hassink, G. C., Zhao, B., Sompallae, R., Altun, M., Gastaldello, S., Zinin, N. V., Masucci, M. G. and Lindsten, K. (2009). The ER-resident ubiquitin-specific protease 19 participates in the UPR and rescues ERAD substrates. EMBO reports 10, 755761.

Helenius, A. and Aebi, M. (2004). Roles of N-Linked Glycans in the Endoplasmic Reticulum. Annual Review of Biochemistry 73, 1019-1049.

Heo, P., Kong, B., Jung, Y.-H., Park, J.-B., Shin, J., Park, M. and Kweon, D.-H. (2017). Green fluorescence protein-based content-mixing assay of SNARE-driven membrane fusion. Biochemical and Biophysical Research Communications 488, 53-59.

Hernandez, J. M., Stein, A., Behrmann, E., Riedel, D., Cypionka, A., Farsi, Z., Walla, P. J., Raunser, S. and Jahn, R. (2012). Membrane Fusion Intermediates via Directional and Full Assembly of the SNARE Complex. Science 336, 1581-1584.

Hetz, C. (2012). The unfolded protein response: controlling cell fate decisions under ER stress and beyond. Nature Reviews Molecular Cell Biology 13, 89-102.

Hill, K., Model, K., Ryan, M. T., Dietmeier, K., Martin, F., Wagner, R. and Pfanner, N. (1998). Tom40 forms the hydrophilic channel of the mitochondrial import pore for preproteins. Nature 395, 516-521.

Hiller, M. M., Finger, A., Schweiger, M. and Wolf, D. H. (1996). ER Degradation of a Misfolded Luminal Protein by the Cytosolic Ubiquitin-Proteasome Pathway. Science 273, 1725-1728.

Hinnah, S. C., Wagner, R., Sveshnikova, N., Harrer, R. and Soll, J. (2002). The Chloroplast Protein Import Channel Toc75: Pore Properties and Interaction with Transit Peptides. Biophysical Journal 83, 899-911.

Hitt, R. and Wolf, D. H. (2004). Der1p, a protein required for degradation of malfolded soluble proteins of the endoplasmic reticulum: topology and Der1-like proteins. FEMS Yeast Research 4, 721-729.

Horn, S. C., Hanna, J., Hirsch, C., Volkwein, C., SchÃCEtz, A., Heinemann, U., Sommer, T. and Jarosch, E. (2009). Usa1 Functions as a Scaffold of the HRD-Ubiquitin Ligase. Molecular Cell 36, 782-793.

Hosokawa, N., Wada, I., Nagasawa, K., Moriyama, T., Okawa, K. and Nagata, K. (2008). Human XTP3-B Forms an Endoplasmic Reticulum Quality Control Scaffold with the HRD1-SEL1L Ubiquitin Ligase Complex and BiP. Journal of Biological Chemistry 283, 20914-20924. 
Hwang, J. and Qi, L. (2018). Quality Control in the Endoplasmic Reticulum: Crosstalk between ERAD and UPR pathways. Trends in Biochemical Sciences 43, 593-605.

Ishikura, S., Weissman, A. M. and Bonifacino, J. S. (2010). Serine Residues in the Cytosolic Tail of the T-cell Antigen Receptor -Chain Mediate Ubiquitination and Endoplasmic Reticulum-associated Degradation of the Unassembled Protein. Journal of Biological Chemistry 285, 23916-23924.

Jahn, R. and Scheller, R. H. (2006). SNAREs - engines for membrane fusion. Nature Reviews Molecular Cell Biology 7, 631-643.

Jakob, C. A., Burda, P., Roth, J. and Aebi, M. (1998). Degradation of Misfolded Endoplasmic Reticulum Glycoproteins inSaccharomyces cerevisiaeIs Determined by a Specific Oligosaccharide Structure. The Journal of Cell Biology 142, 1223-1233.

Jarosch, E., Taxis, C., Volkwein, C., Bordallo, J., Finley, D., Wolf, D. H. and Sommer, T. (2002). Protein dislocation from the ER requires polyubiquitination and the AAA-ATPase Cdc48. Nature Cell Biology 4, 134-139.

Kaganovich, D., Kopito, R. and Frydman, J. (2008). Misfolded proteins partition between two distinct quality control compartments. Nature 454, 1088-1095.

Kanehara, K., Xie, W. and Ng, D. T. (2010). Modularity of the Hrd1 ERAD complex underlies its diverse client range. The Journal of Cell Biology 188, 707-716.

Kario, E., Tirosh, B., Ploegh, H. L. and Navon, A. (2007). N-Linked Glycosylation Does Not Impair Proteasomal Degradation but Affects Class I Major Histocompatibility Complex Presentation. Journal of Biological Chemistry 283, 244-254.

Khouri, E. E., Pavec, G. L., Toledano, M. B. and Delaunay-Moisan, A. (2013). RNF185 Is a Novel E3 Ligase of Endoplasmic Reticulum-associated Degradation (ERAD) That Targets Cystic Fibrosis Transmembrane Conductance Regulator (CFTR). Journal of Biological Chemistry 288, 31177-31191.

Kikkert, M., Doolman, R., Dai, M., Avner, R., Hassink, G., van Voorden, S., Thanedar, S., Roitelman, J., Chau, V. and Wiertz, E. (2004). Human HRD1 Is an E3 Ubiquitin Ligase Involved in Degradation of Proteins from the Endoplasmic Reticulum. Journal of Biological Chemistry 279, 3525-3534.

Kim, I., Mi, K. and Rao, H. (2004). Multiple Interactions of Rad23 Suggest a Mechanism for Ubiquitylated Substrate Delivery Important in Proteolysis. Molecular Biology of the Cell 15, 3357-3365.

Kim, W., Spear, E. D. and Ng, D. T. (2005). Yos9p Detects and Targets Misfolded Glycoproteins for ER-Associated Degradation. Molecular Cell 19, 753-764. 
Kniss, A., Kazemi, S., Lohr, F., Guntert, P. and Dotsch, V. (2019). Solution structure of the RING domain of the E3 ubiquitin ligase HRD1.

Knop, M., Finger, A., Braun, T., Hellmuth, K. and Wolf, D. H. (1996). Der1, a novel protein specifically required for endoplasmic reticulum degradation in yeast. The EMBO Journal 15, 753-763.

Koegl, M., Hoppe, T., Schlenker, S., Ulrich, H. D., Mayer, T. U. and Jentsch, S. (1999). A Novel Ubiquitination Factor, E4, Is Involved in Multiubiquitin Chain Assembly. Cell 96, 635-644.

Komander, D. and Rape, M. (2012). The Ubiquitin Code. Annual Review of Biochemistry $81,203-229$.

Kostova, Z. (2005). Importance of carbohydrate positioning in the recognition of mutated CPY for ER-associated degradation. Journal of Cell Science 118, 1485-1492.

Kostova, Z., Mariano, J., Scholz, S., Koenig, C. and Weissman, A. M. (2009). A Ubc7pbinding domain in Cue1p activates ER-associated protein degradation. Journal of Cell Science 122, 1374-1381.

Kovermann, P., Truscott, K. N., Guiard, B., Rehling, P., Sepuri, N. B., MÃ(Eller, H., Jensen, R. E., Wagner, R. and Pfanner, N. (2002). Tim22, the Essential Core of the Mitochondrial Protein Insertion Complex, Forms a Voltage-Activated and SignalGated Channel. Molecular Cell 9, 363-373.

Krasilnikov, O., Sabirov, R., Ternovsky, V., Merzliak, P. and Muratkhodjaev, J. (1992). A simple method for the determination of the pore radius of ion channels in planar lipid bilayer membranes. FEMS Microbiology Letters 105, 93-100.

Kreft, S. G., Wang, L. and Hochstrasser, M. (2005). Membrane Topology of the Yeast Endoplasmic Reticulum-localized Ubiquitin Ligase Doa10 and Comparison with Its Human Ortholog TEB4 (MARCH-VI). Journal of Biological Chemistry 281, 46464653

Kumazaki, K., Chiba, S., Takemoto, M., Furukawa, A., ichi Nishiyama, K., Sugano, Y., Mori, T., Dohmae, N., Hirata, K., Nakada-Nakura, Y., Maturana, A. D., Tanaka, Y., Mori, H., Sugita, Y., Arisaka, F., Ito, K., Ishitani, R., Tsukazaki, T. and Nureki, O. (2014). Structural basis of Sec-independent membrane protein insertion by YidC. Nature 509, 516-520.

Kyoung, M., Srivastava, A., Zhang, Y., Diao, J., Vrljic, M., Grob, P., Nogales, E., Chu, S. and Brunger, A. T. (2011). In vitro system capable of differentiating fast Ca2- 
triggered content mixing from lipid exchange for mechanistic studies of neurotransmitter release. Proceedings of the National Academy of Sciences 108, E304-E313.

Leto, D. E., Morgens, D. W., Zhang, L., Walczak, C. P., Elias, J. E., Bassik, M. C. and Kopito, R. R. (2019). Genome-wide CRISPR Analysis Identifies SubstrateSpecific Conjugation Modules in ER-Associated Degradation. Molecular Cell 73, 377-389.e11.

Li, S.-J. and Hochstrasser, M. (1999). A new protease required for cell-cycle progression in yeast. Nature 398, 246-251.

Liebermeister, W., Rapoport, T. A. and Heinrich, R. (2001). Ratcheting in posttranslational protein translocation: a mathematical model. Journal of Molecular Biology 305, 643-656.

Lilley, B. N. and Ploegh, H. L. (2004). A membrane protein required for dislocation of misfolded proteins from the ER. Nature 429, 834-840.

Lilley, B. N. and Ploegh, H. L. (2005). Multiprotein complexes that link dislocation, ubiquitination, and extraction of misfolded proteins from the endoplasmic reticulum membrane. Proceedings of the National Academy of Sciences 102, 14296-14301.

Lippe, C. (1969). Urea and thiourea permeabilities of phospholipid and cholesterol bilayer membranes. Journal of Molecular Biology 39, 669-672.

Liu, X., Seven, A. B., Xu, J., Esser, V., Su, L., Ma, C. and Rizo, J. (2017). Simultaneous lipid and content mixing assays for in vitro reconstitution studies of synaptic vesicle fusion. Nature Protocols 12, 2014-2028.

Liu, Y.-C., Fujimori, D. G. and Weissman, J. S. (2016). Htm1p-Pdi1p is a foldingsensitive mannosidase that marks N-glycoproteins for ER-associated protein degradation. Proceedings of the National Academy of Sciences 113, E4015-E4024.

Los, G. V., Encell, L. P., McDougall, M. G., Hartzell, D. D., Karassina, N., Zimprich, C., Wood, M. G., Learish, R., Ohana, R. F., Urh, M., Simpson, D., Mendez, J., Zimmerman, K., Otto, P., Vidugiris, G., Zhu, J., Darzins, A., Klaubert, D. H., Bulleit, R. F. and Wood, K. V. (2008). HaloTag: A Novel Protein Labeling Technology for Cell Imaging and Protein Analysis. ACS Chemical Biology 3, 373-382.

Matlack, K. E., Misselwitz, B., Plath, K. and Rapoport, T. A. (1999). BiP Acts as a Molecular Ratchet during Posttranslational Transport of Prepro- Factor across the ER Membrane. Cell 97, 553-564.

Medicherla, B., Kostova, Z., Schaefer, A. and Wolf, D. H. (2004). A genomic screen 
identifies Dsk2p and Rad23p as essential components of ER-associated degradation. EMBO reports 5, 692-697.

Mehnert, M., Sommer, T. and Jarosch, E. (2013). Der1 promotes movement of misfolded proteins through the endoplasmic reticulum membrane. Nature Cell Biology $16,77-86$.

Mehnert, M., Sommermeyer, F., Berger, M., Lakshmipathy, S. K., Gauss, R., Aebi, M., Jarosch, E. and Sommer, T. (2015). The interplay of Hrd3 and the molecular chaperone system ensures efficient degradation of malfolded secretory proteins. Molecular Biology of the Cell 26, 185-194.

Mehrtash, A. B. and Hochstrasser, M. (2018). Ubiquitin-dependent protein degradation at the endoplasmic reticulum and nuclear envelope. Seminars in Cell \& Developmental Biology https://doi.org/10.1016/j.semcdb.2018.09.013, in press.

Meinecke, M., Cizmowski, C., Schliebs, W., Krueger, V., Beck, S., Wagner, R. and Erdmann, R. (2010). The peroxisomal importomer constitutes a large and highly dynamic pore. Nature Cell Biology 12, 273-277.

Meinecke, M., Wagner, R., Kovermann, P., Guiard, B., Mick, D. U., Hutu, D. P., Voos, W., Truscott, K. N., Chacinska, A., Pfanner, N. and Rehling, P. (2006). Tim50 Maintains the Permeability Barrier of the Mitochondrial Inner Membrane. Science 312, 1523-1526.

Menzies, S. A., Volkmar, N., van den Boomen, D. J., Timms, R. T., Dickson, A. S., Nathan, J. A. and Lehner, P. J. (2018). The sterol-responsive RNF145 E3 ubiquitin ligase mediates the degradation of HMG-CoA reductase together with gp78 and Hrd1. eLife $\%$.

Metzger, M. B., Liang, Y.-H., Das, R., Mariano, J., Li, S., Li, J., Kostova, Z., Byrd, R. A., Ji, X. and Weissman, A. M. (2013). A Structurally Unique E2-Binding Domain Activates Ubiquitination by the ERAD E2, Ubc7p, through Multiple Mechanisms. Molecular Cell 50, 516-527.

Miller, J. H. (1992). A Short Course in Bacterial Genetics. Cold Spring Harbor Laboratory Press.

Mittl, P. R. and Schneider-Brachert, W. (2007). Sel1-like repeat proteins in signal transduction. Cellular Signalling 19, 20-31.

Morito, D., Hirao, K., Oda, Y., Hosokawa, N., Tokunaga, F., Cyr, D. M., Tanaka, K., Iwai, K. and Nagata, K. (2008). Gp78 cooperates with RMA1 in endoplasmic 
reticulum-associated degradation of CFTRDeltaF508. Molecular Biology of the Cell 19, 1328-1336.

Morito, D. and Nagata, K. (2015). Pathogenic Hijacking of ER-Associated Degradation: Is ERAD Flexible? Molecular Cell 59, 335-344.

Mueller, B., Klemm, E. J., Spooner, E., Claessen, J. H. and Ploegh, H. L. (2008). SEL1L nucleates a protein complex required for dislocation of misfolded glycoproteins. Proceedings of the National Academy of Sciences 105, 12325-12330.

Mueller, B., Lilley, B. N. and Ploegh, H. L. (2006). SEL1L, the homologue of yeast Hrd3p, is involved in protein dislocation from the mammalian ER. The Journal of Cell Biology 175, 261-270.

Mukaiyama, H., Kodera, M., Tanaka, N. and Takegawa, K. (2011). N-glycans are not required for the efficient degradation of the mutant Saccharomyces cerevisiae CPY*inSchizosaccharomycespombe.AppliedMicrobiologyandBiotechnology 93, 1609-1618 .

Mumberg, D., Mueller, R. and Funk, M. (1994). Regulatable promoters of Saccharomyces cerevisiae: comparison of transcriptional activity and their use for heterologous expression. Nucleic acids research 22, 5767-5768.

Neal, S., Jaeger, P. A., Duttke, S. H., Benner, C., K.Glass, C., Ideker, T. and Hampton, R. Y. (2018). The Dfm1 Derlin Is Required for ERAD Retrotranslocation of Integral Membrane Proteins. Molecular Cell 69, 306-320.e4.

Neal, S., Mak, R., Bennett, E. J. and Hampton, R. (2017). A Cdc48 "Retrochaperone" Function Is Required for the Solubility of Retrotranslocated, Integral Membrane Endoplasmic Reticulum-associated Degradation (ERAD-M) Substrates. Journal of Biological Chemistry 292, 3112-3128.

Needham, P. G., Guerriero, C. J. and Brodsky, J. L. (2019). Chaperoning Endoplasmic Reticulum-Associated Degradation (ERAD) and Protein Conformational Diseases. Cold Spring Harbor Perspectives in Biology doi/10.1101/cshperspect.a033928, a033928.

Neklesa, T. K., Tae, H. S., Schneekloth, A. R., Stulberg, M. J., Corson, T. W., Sundberg, T. B., Raina, K., Holley, S. A. and Crews, C. M. (2011). Small-molecule hydrophobic tagging-induced degradation of HaloTag fusion proteins. Nature Chemical Biology 7, 538-543.

Neuber, O., Jarosch, E., Volkwein, C., Walter, J. and Sommer, T. (2005). Ubx2 links 
the Cdc48 complex to ER-associated protein degradation. Nature Cell Biology \%, 993-998.

Nezil, F. and Bloom, M. (1992). Combined influence of cholesterol and synthetic amphiphillic peptides upon bilayer thickness in model membranes. Biophysical Journal $61,1176-1183$.

Nickel, W., Weber, T., McNew, J. A., Parlati, F., Soellner, T. H. and Rothman, J. E. (1999). Content mixing and membrane integrity during membrane fusion driven by pairing of isolated v-SNAREs and t-SNAREs. Proceedings of the National Academy of Sciences of the United States of America 96, 12571-12576.

Niles, W. D., Cohen, F. S. and Finkelstein, A. (1989). Hydrostatic pressures developed by osmotically swelling vesicles bound to planar membranes. The Journal of General Physiology 93, 211-244.

Nishikawa, S., Fewell, S. W., Kato, Y., Brodsky, J. L. and Endo, T. (2001). Molecular Chaperones in the Yeast Endoplasmic Reticulum Maintain the Solubility of Proteins for Retrotranslocation and Degradation. The Journal of Cell Biology 153, 1061-1070.

Nordlund, G., Brzezinski, P. and von Ballmoos, C. (2014). SNARE-fusion mediated insertion of membrane proteins into native and artificial membranes. Nature Communications 5 .

Nussinov, R., Tsai, C.-J., Xin, F. and Radivojac, P. (2012). Allosteric post-translational modification codes. Trends in Biochemical Sciences 37, 447-455.

Oda, Y., Okada, T., Yoshida, H., Kaufman, R. J., Nagata, K. and Mori, K. (2006). Derlin-2 and Derlin-3 are regulated by the mammalian unfolded protein response and are required for ER-associated degradation. The Journal of Cell Biology 172, 383-393.

Okuda-Shimizu, Y. and Hendershot, L. M. (2007). Characterization of an ERAD Pathway for Nonglycosylated BiP Substrates, which Require Herp. Molecular Cell 28, 544-554.

Osborne, A. R. and Rapoport, T. A. (2007). Protein Translocation Is Mediated by Oligomers of the SecY Complex with One SecY Copy Forming the Channel. Cell 129, $97-110$.

Parodi, A. J. (2000). Protein Glucosylation and Its Role in Protein Folding. Annual Review of Biochemistry 69, 69-93.

Pelham, H. R., Hardwick, K. G. and Lewis, M. J. (1988). Sorting of soluble ER proteins in yeast. The EMBO journal 7, 1757-1762. 
Pickart, C. M. (2001). Mechanisms Underlying Ubiquitination. Annual Review of Biochemistry 70, 503-533.

Pilon, M. (1997). Sec61p mediates export of a misfolded secretory protein from the endoplasmic reticulum to the cytosol for degradation. The EMBO Journal 16, 45404548 .

Plemper, R. K., Bohmler, S., Bordallo, J., Sommer, T. and Wolf, D. H. (1997). Mutant analysis links the translocon and BiP to retrograde protein transport for ER degradation. Nature 388, 891-895.

Plemper, R. K., Bordallo, J., Deak, P. M., Taxis, C., Hitt, R. and Wolf, D. H. (1999). Genetic interactions of Hrd3p and Der3p/Hrd1p with Sec61p suggest a retrotranslocation complex mediating protein transport for ER degradation. Journal of Cell Science 112 ( Pt 22), 4123-4134.

Pobbati, A. V., Stein, A. and Fasshauer, D. (2006). N- to C-Terminal SNARE Complex Assembly Promotes Rapid Membrane Fusion. Science 313, 673-676.

Popp, M. W.-L. and Ploegh, H. L. (2011). Making and Breaking Peptide Bonds: Protein Engineering Using Sortase. Angewandte Chemie International Edition 50, $5024-5032$.

Quan, E. M., Kamiya, Y., Kamiya, D., Denic, V., Weibezahn, J., Kato, K. and Weissman, J. S. (2008). Defining the Glycan Destruction Signal for Endoplasmic Reticulum-Associated Degradation. Molecular Cell 32, 870-877.

Rabinovich, E., Kerem, A., Frohlich, K.-U., Diamant, N. and Bar-Nun, S. (2002). AAAATPase p97/Cdc48p, a Cytosolic Chaperone Required for Endoplasmic ReticulumAssociated Protein Degradation. Molecular and Cellular Biology 22, 626-634.

Raina, K., Noblin, D. J., Serebrenik, Y. V., Adams, A., Zhao, C. and Crews, C. M. (2014). Targeted protein destabilization reveals an estrogen-mediated ER stress response. Nature Chemical Biology 10, 957-962.

Ramadan, K., Bruderer, R., Spiga, F. M., Popp, O., Baur, T., Gotta, M. and Meyer, H. H. (2007). Cdc48/p97 promotes reformation of the nucleus by extracting the kinase Aurora B from chromatin. Nature 450, 1258-1262.

Rape, M., Hoppe, T., Gorr, I., Kalocay, M., Richly, H. and Jentsch, S. (2001). Mobilization of Processed, Membrane-Tethered SPT23 Transcription Factor by CDC48UFD1/NPL4, a Ubiquitin-Selective Chaperone. Cell 10\%, 667-677.

Rapoport, T. A., Li, L. and Park, E. (2017). Structural and Mechanistic Insights 
into Protein Translocation. Annual Review of Cell and Developmental Biology 33, 369-390.

Ravid, T. and Hochstrasser, M. (2008). Diversity of degradation signals in the ubiquitin-proteasome system. Nature Reviews Molecular Cell Biology 9, 679-689.

Reinhold, R., Kruger, V., Meinecke, M., Schulz, C., Schmidt, B., Grunau, S. D., Guiard, B., Wiedemann, N., van der Laan, M., Wagner, R., Rehling, P. and Dudek, J. (2012). The Channel-Forming Sym1 Protein Is Transported by the TIM23 Complex in a Presequence-Independent Manner. Molecular and Cellular Biology 32, 50095021.

Renatus, M., Parrado, S. G., D'Arcy, A., Eidhoff, U., Gerhartz, B., Hassiepen, U., Pierrat, B., Riedl, R., Vinzenz, D., Worpenberg, S. and Kroemer, M. (2006). Structural Basis of Ubiquitin Recognition by the Deubiquitinating Protease USP2. Structure 14, 1293-1302.

Richly, H., Rape, M., Braun, S., Rumpf, S., Hoege, C. and Jentsch, S. (2005). A Series of Ubiquitin Binding Factors Connects CDC48/p97 to Substrate Multiubiquitylation and Proteasomal Targeting. Cell 120, 73-84.

Rigaud, J.-L. and Lévy, D. (2003). Reconstitution of Membrane Proteins into Liposomes. In Methods in Enzymology pp. 65-86. Elsevier.

Rodrigo-Brenni, M. C. and Morgan, D. O. (2007). Sequential E2s Drive Polyubiquitin Chain Assembly on APC Targets. Cell 130, 127-139.

Rosenbaum, J. C., Fredrickson, E. K., Oeser, M. L., Garrett-Engele, C. M., Locke, M. N., Richardson, L. A., Nelson, Z. W., Hetrick, E. D., Milac, T. I., Gottschling, D. E. and Gardner, R. G. (2011). Disorder Targets Misorder in Nuclear Quality Control Degradation: A Disordered Ubiquitin Ligase Directly Recognizes Its Misfolded Substrates. Molecular Cell 41, 93-106.

Ruffieux-Daidié, D. and Staub, O. (2010). Intracellular Ubiquitylation of the Epithelial NaChannel Controls Extracellular Proteolytic Channel Activation via Conformational Change. Journal of Biological Chemistry 286, 2416-2424.

Ruggiano, A., Foresti, O. and Carvalho, P. (2014). ER-associated degradation: Protein quality control and beyond. The Journal of Cell Biology 204, 869-879.

Samant, R. S., Livingston, C. M., Sontag, E. M. and Frydman, J. (2018). Distinct proteostasis circuits cooperate in nuclear and cytoplasmic protein quality control. Nature 563, 407-411. 
Saparov, S. M., Erlandson, K., Cannon, K., Schaletzky, J., Schulman, S., Rapoport, T. A. and Pohl, P. (2007). Determining the Conductance of the SecY Protein Translocation Channel for Small Molecules. Molecular Cell 26, 501-509.

Sato, B. K. and Hampton, R. Y. (2006). Yeast Derlin Dfm1 interacts with Cdc48 and functions in ER homeostasis. Yeast 23, 1053-1064.

Sato, B. K., Schulz, D., Do, P. H. and Hampton, R. Y. (2009). Misfolded Membrane Proteins Are Specifically Recognized by the Transmembrane Domain of the Hrd1p Ubiquitin Ligase. Molecular Cell 34, 212-222.

Schaefer, A. and Wolf, D. H. (2009). Sec61p is part of the endoplasmic reticulumassociated degradation machinery. The EMBO Journal 28, 2874-2884.

Schaegger, H. (2006). Tricine-SDS-PAGE. Nature Protocols 1, 16-22.

Schneider, K. L., Nystrom, T. and Widlund, P. O. (2018). Studying Spatial Protein Quality Control, Proteopathies, and Aging Using Different Model Misfolding Proteins in S. cerevisiae. Frontiers in Molecular Neuroscience 11.

Schoebel, S., Mi, W., Stein, A., Ovchinnikov, S., Pavlovicz, R., DiMaio, F., Baker, D., Chambers, M. G., Su, H., Li, D., Rapoport, T. A. and Liao, M. (2017). CryoEM structure of the protein-conducting ERAD channel Hrd1 in complex with Hrd3. Nature 548, 352-355.

Schuberth, C. and Buchberger, A. (2005). Membrane-bound Ubx2 recruits Cdc48 to ubiquitin ligases and their substrates to ensure efficient ER-associated protein degradation. Nature Cell Biology 7, 999-1006.

Schuberth, C., Richly, H., Rumpf, S. and Buchberger, A. (2004). Shp1 and Ubx2 are adaptors of Cdc48 involved in ubiquitin-dependent protein degradation. EMBO reports $5,818-824$.

Schulz, J., Avci, D., Queisser, M. A., Gutschmidt, A., Dreher, L.-S., Fenech, E. J., Volkmar, N., Hayashi, Y., Hoppe, T. and Christianson, J. C. (2017). Conserved cytoplasmic domains promote Hrd1 ubiquitin ligase complex formation for ER-associated degradation (ERAD). Journal of Cell Science 130, 3322-3335.

Schulze, A., Standera, S., Buerger, E., Kikkert, M., van Voorden, S., Wiertz, E., Koning, F., Kloetzel, P.-M. and Seeger, M. (2005). The Ubiquitin-domain Protein HERP forms a Complex with Components of the Endoplasmic Reticulum Associated Degradation Pathway. Journal of Molecular Biology 354, 1021-1027.

Shimizu, Y., Okuda-Shimizu, Y. and Hendershot, L. M. (2010). Ubiquitylation of an 
ERAD Substrate Occurs on Multiple Types of Amino Acids. Molecular Cell 40, 917-926.

Smart, O., Breed, J., Smith, G. and Sansom, M. (1997). A novel method for structurebased prediction of ion channel conductance properties. Biophysical Journal 72, $1109-1126$.

Sommer, T. and Jentsch, S. (1993). A protein translocation defect linked to ubiquitin conjugation at the endoplasmic reticulum. Nature 365, 176-179.

Spear, E. D. and Ng, D. T. (2005). Single, context-specific glycans can target misfolded glycoproteins for ER-associated degradation. The Journal of Cell Biology 169, 73-82.

Stanley, A. M., Carvalho, P. and Rapoport, T. (2011). Recognition of an ERAD-L substrate analyzed by site-specific in vivo photocrosslinking. FEBS Letters 585, $1281-1286$.

Stefanovic-Barrett, S., Dickson, A. S., Burr, S. P., Williamson, J. C., Lobb, I. T., van den Boomen, D. J., Lehner, P. J. and Nathan, J. A. (2018). MARCH6 and TRC8 facilitate the quality control of cytosolic and tail-anchored proteins. EMBO reports $19, \mathrm{e} 45603$.

Stein, A., Radhakrishnan, A., Riedel, D., Fasshauer, D. and Jahn, R. (2007). Synaptotagmin activates membrane fusion through a Ca2-dependent trans interaction with phospholipids. Nature Structural \& Molecular Biology 14, 904-911.

Stein, A., Ruggiano, A., Carvalho, P. and Rapoport, T. A. (2014). Key Steps in ERAD of Luminal ER Proteins Reconstituted with Purified Components. Cell 158, $1375-1388$.

Stewart, M. D., Ritterhoff, T., Klevit, R. E. and Brzovic, P. S. (2016). E2 enzymes: more than just middle men. Cell Research 26, 423-440.

Stolz, A., Schweizer, R. S., SchÃ€fer, A. and Wolf, D. H. (2010). Dfm1 Forms Distinct Complexes with Cdc48 and the ER Ubiquitin Ligases and Is Required for ERAD. Traffic 11, 1363-1369.

Struck, D. K., Hoekstra, D. and Pagano, R. E. (1981). Use of resonance energy transfer to monitor membrane fusion. Biochemistry 20, 4093-4099.

Suzuki, T., Huang, C. and Fujihira, H. (2016). The cytoplasmic peptide:N-glycanase (NGLY1) - Structure, expression and cellular functions. Gene 577, 1-7.

Swanson, R., Locher, M. and Hochstrasser, M. (2001). A conserved ubiquitin ligase of the nuclear envelope/endoplasmic reticulum that functions in both ER-associated and Matalpha 2 repressor degradation. Genes \& Development 15, 2660-2674. 
Szathmary, R., Bielmann, R., Nita-Lazar, M., Burda, P. and Jakob, C. A. (2005). Yos9 Protein Is Essential for Degradation of Misfolded Glycoproteins and May Function as Lectin in ERAD. Molecular Cell 19, 765-775.

Tartoff, K. D. and Hobbs, C. A. (1987). Improved media for growing plasmid and cosmid clones. Bethesda Research Labs Focus 9, 2-12.

Taylor, E. B. and Rutter, J. (2011). Mitochondrial quality control by the ubiquitin-proteasome system: Figure 1. Biochemical Society Transactions 39, 1509-1513.

Teese, M. G. and Langosch, D. (2015). Role of GxxxG Motifs in Transmembrane Domain Interactions. Biochemistry 54, 5125-5135.

Theesfeld, C. L. and Hampton, R. Y. (2013). Insulin-induced Gene Protein (INSIG)dependent Sterol Regulation of Hmg2 Endoplasmic Reticulum-associated Degradation (ERAD) in Yeast. Journal of Biological Chemistry 288, 8519-8530.

Truscott, K. N., Kovermann, P., Geissler, A., Merlin, A., Meijer, M., Driessen, A. J., Rassow, J., Pfanner, N. and Wagner, R. (2001). A presequence- and voltage-sensitive channel of the mitochondrial preprotein translocase formed by Tim23. Nature Structural Biology 8, 1074-1082.

van de Weijer, M. L., Bassik, M. C., Luteijn, R. D., Voorburg, C. M., Lohuis, M. A., Kremmer, E., Hoeben, R. C., LeProust, E. M., Chen, S., Hoelen, H., Ressing, M. E., Patena, W., Weissman, J. S., McManus, M. T., Wiertz, E. J. and Lebbink, R. J. (2014). A high-coverage shRNA screen identifies TMEM129 as an E3 ligase involved in ER-associated protein degradation. Nature Communications 5.

van den Berg, B., Clemons, W. M., Collinson, I., Modis, Y., Hartmann, E., Harrison, S. C. and Rapoport, T. A. (2004). X-ray structure of a protein-conducting channel. Nature 427, 36-44.

van den Bogaart, G., Holt, M. G., Bunt, G., Riedel, D., Wouters, F. S. and Jahn, R. (2010). One SNARE complex is sufficient for membrane fusion. Nature Structural \& Molecular Biology 17, 358-364.

van den Boomen, D. J. H., Timms, R. T., Grice, G. L., Stagg, H. R., Skodt, K., Dougan, G., Nathan, J. A. and Lehner, P. J. (2014). TMEM129 is a Derlin-1 associated ERAD E3 ligase essential for virus-induced degradation of MHC-I. Proceedings of the National Academy of Sciences 111, 11425-11430.

Vashist, S. and Ng, D. T. (2004). Misfolded proteins are sorted by a sequential checkpoint mechanism of ER quality control. The Journal of Cell Biology 165, 41-52. 
Vashistha, N., Neal, S. E., Singh, A., Carroll, S. M. and Hampton, R. Y. (2016). Direct and essential function for Hrd3 in ER-associated degradation. Proceedings of the National Academy of Sciences 113, 5934-5939.

Verma, R., Oania, R. S., Kolawa, N. J. and Deshaies, R. J. (2013). Cdc48/p97 promotes degradation of aberrant nascent polypeptides bound to the ribosome. eLife 2.

von Delbrueck, M., Kniss, A., Rogov, V. V., Pluska, L., Bagola, K., Lohr, F., Guentert, P., Sommer, T. and Dotsch, V. (2016). The CUE Domain of Cue1 Aligns Growing Ubiquitin Chains with Ubc7 for Rapid Elongation. Molecular Cell 62, 918-928.

Wahlman, J., DeMartino, G. N., Skach, W. R., Bulleid, N. J., Brodsky, J. L. and Johnson, A. E. (2007). Real-Time Fluorescence Detection of ERAD Substrate Retrotranslocation in a Mammalian In Vitro System. Cell 129, 943-955.

Walter, P. and Ron, D. (2011). The Unfolded Protein Response: From Stress Pathway to Homeostatic Regulation. Science 334, 1081-1086.

Wang, Y., Zhang, Y. and Ha, Y. (2006). Crystal structure of a rhomboid family intramembrane protease. Nature 444, 179-180.

Wangeline, M. A. and Hampton, R. Y. (2018). "Mallostery"-ligand-dependent protein misfolding enables physiological regulation by ERAD. Journal of Biological Chemistry 293, 14937-14950.

Weber, A., Cohen, I., Popp, O., Dittmar, G., Reiss, Y., Sommer, T., Ravid, T. and Jarosch, E. (2016). Sequential Poly-ubiquitylation by Specialized Conjugating Enzymes Expands the Versatility of a Quality Control Ubiquitin Ligase. Molecular Cell 63, 827-839.

Werner, E. D., Brodsky, J. L. and McCracken, A. A. (1996). Proteasome-dependent endoplasmic reticulum-associated protein degradation: An unconventional route to a familiar fate. Proceedings of the National Academy of Sciences 93, 13797-13801.

Wiedemann, N. and Pfanner, N. (2017). Mitochondrial Machineries for Protein Import and Assembly. Annual Review of Biochemistry 86, 685-714.

Wiertz, E. J. H. J., Tortorella, D., Bogyo, M., Yu, J., Mothes, W., Jones, T. R., Rapoport, T. A. and Ploegh, H. L. (1996). Sec6l-mediated transfer of a membrane protein from the endoplasmic reticulum to the proteasome for destruction. Nature 384, 432-438.

Wilkinson, C. R., Seeger, M., Hartmann-Petersen, R., Stone, M., Wallace, M., Semple, C. and Gordon, C. (2001). Proteins containing the UBA domain are able to bind to multi-ubiquitin chains. Nature Cell Biology 3, 939-943. 
Wilkinson, K. A. and Henley, J. M. (2010). Mechanisms, regulation and consequences of protein SUMOylation. Biochemical Journal 428, 133-145.

Willer, M., Forte, G. M. A. and Stirling, C. J. (2008). Sec61p Is Required for ERAD-L. Journal of Biological Chemistry 283, 33883-33888.

Winget, J. M. and Mayor, T. (2010). The Diversity of Ubiquitin Recognition: Hot Spots and Varied Specificity. Molecular Cell 38, 627-635.

Wirth, A., Jung, M., Bies, C., Frien, M., Tyedmers, J., Zimmermann, R. and Wagner, R. (2003). The Sec61p Complex Is a Dynamic Precursor Activated Channel. Molecular Cell 12, 261-268.

Wu, X., Cabanos, C. and Rapoport, T. A. (2019). Structure of the post-translational protein translocation machinery of the ER membrane. Nature 566, 136-139.

Wu, Z., Yan, N., Feng, L., Oberstein, A., Yan, H., Baker, R. P., Gu, L., Jeffrey, P. D., Urban, S. and Shi, Y. (2006). Structural analysis of a rhomboid family intramembrane protease reveals a gating mechanism for substrate entry. Nature Structural \& Molecular Biology 13, 1084-1091.

$\mathrm{Xu}$, C. and Ng, D. T. W. (2015). Glycosylation-directed quality control of protein folding. Nature Reviews Molecular Cell Biology 16, 742-752.

Ye, Y., Meyer, H. H. and Rapoport, T. A. (2001). The AAA ATPase Cdc48/p97 and its partners transport proteins from the ER into the cytosol. Nature 414, 652-656.

Ye, Y., Meyer, H. H. and Rapoport, T. A. (2003). Function of the p97-Ufd1-Npl4 complex in retrotranslocation from the ER to the cytosol. The Journal of Cell Biology 162, 71-84.

Ye, Y., Shibata, Y., Yun, C., Ron, D. and Rapoport, T. A. (2004). A membrane protein complex mediates retro-translocation from the ER lumen into the cytosol. Nature 429, 841-847.

Zattas, D. and Hochstrasser, M. (2014). Ubiquitin-dependent protein degradation at the yeast endoplasmic reticulum and nuclear envelope. Critical Reviews in Biochemistry and Molecular Biology 50, 1-17.

Zheng, N. and Shabek, N. (2017). Ubiquitin Ligases: Structure, Function, and Regulation. Annual Review of Biochemistry 86, 129-157.

Zheng, N., Wang, P., Jeffrey, P. D. and Pavletich, N. P. (2000). Structure of a cCbl-UbcH7 Complex. Cell 102, 533-539. 